\author{
Monograph \\ urn:lsid:zoobank.org:pub:5F29A1A0-CC67-488A-B8A9-98DF1EE4F1E8
}

\title{
A subjective global checklist of the extant non-marine Ostracoda (Crustacea)
}

\author{
Claude MEISCH ${ }^{1, *}$, Robin J. SMITH ${ }^{2}$ \& Koen MARTENS ${ }^{3}$ \\ ${ }^{1}$ National Natural History Museum of Luxembourg, 25, rue Münster, \\ 2160 Luxembourg, Luxembourg. \\ ${ }^{2}$ Lake Biwa Museum, Oroshimo 1091, Kusatsu, Shiga Prefecture 525-0001, Japan. \\ ${ }^{3}$ Royal Belgian Institute of Natural Sciences, OD Nature, Freshwater Biology, \\ Vautierstraat 29, 1000 Brussels, Belgium and University of Ghent, \\ Department of Biology, K.L. Ledeganckstraat 35, 9000 Ghent, Belgium. \\ ${ }^{*}$ Corresponding author: claude.meisch@education.lu \\ 2Email: robin-james-smith@biwahaku.jp \\ ${ }_{3}^{3}$ Email: k.martens@naturalsciences.be,darwinula@gmail.com \\ ${ }^{1}$ urn:1sid:zoobank.org:author:3638C82F-AD2D-4920-99C0-43925994A77F \\ ${ }^{2}$ urn:1sid:zoobank.org:author:D446D7FD-97B8-491A-8AC4-B17809AAC769 \\ ${ }^{3}$ urn:lsid:zoobank.org:author:9272757B-A9E5-4C94-B28D-F5EFF32AADC7
}

\begin{abstract}
We present an updated, subjective list of the extant, non-marine ostracod genera and species of the world, with their distributions in the major zoogeographical regions, as well as a list of the genera in their present hierarchical taxonomic positions. The list includes all taxa described and taxonomic alterations made up to 1 July 2018. Taxonomic changes include 17 new combinations, 5 new names, 1 emended specific name and 11 new synonymies ( 1 tribe, 4 genera, 6 species). Taking into account the recognized synonymies, there are presently 2330 subjective species of non-marine ostracods in 270 genera. The most diverse family in non-marine habitats is the Cyprididae, comprising $43.2 \%$ of all species, followed by the Candonidae (29.0\%), Entocytheridae $(9.1 \%)$ and the Limnocytheridae (7.0\%). An additional 13 families comprise the remaining $11.8 \%$ of described species. The Palaearctic zoogeographical region has the greatest number of described species (799), followed by the Afrotropical region with 453 species and the Nearctic region with 439 species. The Australasian and Neotropical regions each have 328 and 333 recorded species, respectively, while the Oriental region has 271 . The vast majority of non-marine ostracods (89.8\%) are endemic to one zoogeographical region, while only six species are found in six or more regions. We also present an additional list with 'uncertain species', which have neither been redescribed nor re-assessed since 1912, and which are excluded from the main list; a list of taxonomic changes presented in the present paper; a table with the number of species and \% per family; and a table with numbers of new species described in the 20-year period between 1998 and 2017 per zoogeographical region. Two figures visualize the total number of species and endemic species per zoogeographical region, and the numbers of new species descriptions per decade for all families and the three largest families since 1770, respectively.
\end{abstract}

Key words. Ostracoda, distribution, biodiversity, taxonomy, nomenclature. 
Meisch C., Smith R.J. \& Martens K. 2019. A subjective global checklist of the extant non-marine Ostracoda (Crustacea). European Journal of Taxonomy 492: 1-135. https://doi.org/10.5852/ejt.2019/492

\section{Introduction}

The present checklist is an expanded, updated and corrected version of the list published by Martens \& Savatenalinton (2011), which included all the extant free-living non-marine species of ostracods described up to 2008. The present list is expanded to include the species of the Entocytheridae Hoff, 1942, which live as epibionts on the body and appendages of other crustaceans, including crayfish, crabs, amphipods and isopods (Hart \& Hart 1974; Mestre et al. 2014; Williams \& Weaver 2018). The present list is also updated, as it now includes all taxa described and all taxonomic alterations, as well as all faunistic records made up to 1 July 2018. The present list also corrects earlier omissions and errors. In addition, some further taxonomic decisions are taken here and briefly commented on regarding new combinations of species and genus names, new synonymies and new names, when it became apparent that such changes were necessary.

\section{Material and methods}

The checklist by Martens \& Savatenalinton (2011) was rather inclusive, also containing poorly-known species of the $18^{\text {th }}$ and above all the $19^{\text {th }}$ centuries. For the present list, the comprehensive synopsis of the Ostracoda of the world by G.W. Müller (1912, reprint of 1966) is used as a taxonomic filter: the species already considered of dubious status therein and, for most of them, unrecorded since their first description, are discarded from the present list. They are moved to a separate list with short remarks on their taxonomic status. Candona lactea (Baird, 1850) and Potamocypris helicina (Fischer, 1847) are examples of the application of this filter. G.W. Müller (op. cit.) commented on the first of these species as: "larva?". His remark about the second species reads: "insufficient description of a known species?". Exceptions are made for those species which, although retained by the filter, were studied and redescribed after 1912.

For synonymies proposed after 1912, the same guideline already used in the previous checklist (Martens \& Savatenalinton 2011) is applied: synonymies should preferably be based on investigations of type (or at least topotypical) material or on descriptions only if they are exhaustive and detailed. Furthermore, as a rule, proposed synonymies that stretch over two or more zoogeographical regions are not adopted in the list. An example is the proposed synonymy of Candona inexpecta Chapman, 1963 from New Zealand (AU) and Pseudocandona albicans (Brady, 1864) from the Palaearctic (Karanovic 2005).

The distinction between non-marine and marine species is mostly quite straightforward. However, in some specific cases doubts are justified. The present list generally follows the same criteria as applied by Martens et al. (2008) and Martens \& Savatenalinton (2011): (1) if a species is included in the compendiums of non-marine ostracods (Kempf 1980a-d, 1997a-d, 2006) it is accepted here as a non-marine species, unless otherwise indicated; (2) the definition of brackish water species follows the introductory chapter of the 'Freshwater Animal Diversity Assessment' (Balian et al. 2008), i.e., "only the true non-marine fauna from interface environments (estuaries, anchialine ponds) are included and euryhaline species are included if they show a genuine tolerance to freshwater (salinity $<3 \mathrm{~g} / \mathrm{l}$ )."

Trinomina either represent subspecies or varieties (or forms). To distinguish between these two clearly different taxonomic entities (see Article 45 of the International Code of Zoological Nomenclature, ICZN) the following rule is applied: trinomina of subspecies remain unmarked, while those of varieties and forms are marked with 'var.' or 'f.', respectively. In many cases, subspecies will eventually turn out to be valid species. This could be the case for most of the subspecies described from Lake Baikal (Mazepova 
1990). It should be noted that, unlike names of subspecies, names of varieties are not regulated by the ICZN, with the exception, however, of those introduced before 1961 (Article 45.5-45.6 of the ICZN).

The main part of this checklist, the alphabetic list of genera and species, provides the following information:

- valid species and genus names, with author(s) and year of both the original description and, if applicable, the currently accepted generic combination.

- the original generic combination of species, if different from the present one.

- bibliographic references for synonyms of species.

- trinomina for subspecies and varieties or forms, the former unmarked, the latter marked with 'var.' and 'f.', respectively.

- a list of synonyms of genera and species.

- type species of genera as marked with an '*'.

Occurrence of species in zoogeographical regions: the borders of the different regions are as described and illustrated in Balian et al. (2008), but with the boundary between the PA and NA regions through the Bering Strait and in the North Pacific adjusted, as in Fig. 1.

With regard to the zoogeographical information, the following abbreviations apply:

$$
\begin{aligned}
& \text { ANT }=\text { Antarctic } \\
& \text { AT }=\text { Afrotropical } \\
& \text { AU }=\text { Australasian } \\
& \text { NA }=\text { Nearctic } \\
& \text { NT }=\text { Neotropical } \\
& \text { OL }=\text { Oriental } \\
& \text { PA }=\text { Palaearctic } \\
& \text { PAC }=\text { Pacific Oceanic Islands }
\end{aligned}
$$

\section{Results}

The checklist contains 2330 subjective species (not counting subspecies and varieties) of extant, nonmarine Ostracoda of the world, in 270 genera, 17 families and four superfamilies (Table 2). The majority of the species belong to the superfamily Cypridoidea (75.5\%), followed by the Cytheroidea (22.8\%) and Darwinuloidea (1.5\%) (Table 2). The most diverse family in non-marine habitats is the Cyprididae, comprising $43.2 \%$ of all species, followed by the Candonidae (29.0\%), Entocytheridae $(9.1 \%)$ and the Limnocytheridae (7.0\%) (Table 2). The other 13 families comprise the remaining 11.8\% of the species, and of these, eight are primarily marine families with restricted numbers of non-marine representatives. Candona is the most diverse genus with 145 species, followed by Strandesia (97), Pseudocandona (72) and Cypridopsis (66). Eighty-two genera (30.4\%) are monospecific. Two hundred and thirty species (9.9\% of the total) have at least one synonym, while 722 species $(31.0 \%)$ have been transferred to different genera at least once since their initial descriptions (Table 2).

Fifty-nine species have been excluded from the main list as 'uncertain species'. We present 17 new combinations, 5 new names, 1 emended specific name and 11 new synonymies ( 1 tribe, 4 genera, 6 species) (Table 1). Numbers of non-marine species/subspecies and percentages of total number in superfamilies and families are given in Table 2. Numbers of new species described in the 20-year period between 1998 and 2017 in the different zoogeographical regions are presented in Table 3. Figure 1 shows the numbers of non-marine ostracod species reported in each zoogeographical region, with endemic species in parentheses (top), and proportions of species in non-marine families in each region (bottom). Figure 2 shows numbers of new species of non-marine ostracods described per decade for all families and for the largest three families. 


\section{Taxonomic notes (synopsis in Table 1)}

(1) The tribe Danielocandonini Karanovic, 2007 is not adopted here for the following reasons: (a) the genera included are morphologically very diverse, with some of the characters almost certainly being the result of convergent evolution and (b) the tribe stretches, with large geographical gaps, from the Caribbean realm (genus Danielocandona Broodbakker, 1983) to Africa (genus Meischcandona Karanovic, 2007) and Australia (genera Deminutiocandona Karanovic, 2003, Kencandona Karanovic, 2007 and Leicacandona Karanovic, 2007).

(2) The treatment of the genera Typhlocypris Vejdovský, 1882, Pseudocandona Kaufmann, 1900 and Marmocandona Danielopol et al., 2012 follows Danielopol et al. (2012) and Namiotko et al. (2014).

(3) The genus Neglecandona Krstić, 2007, with Neglecandona lindneri (Petkovski, 1969) as the type species, was established to include the species of the so-called neglecta-group of Candona Baird, 1845. Although the genus appears sound and accurately diagnosed (Krstić 2006; Medici et al. 2011), its specific content appears incomplete at present, with only three species of the neglecta-group having so far been formally transferred to Neglecandona. As this needs to be discussed elsewhere, no attempt is made here to transfer any other species of the neglecta-group to that genus.

(4) Cypris subglobosa Sowerby, 1840 was first described from fossil material recovered from Upper Cretaceous sediments. However, it is now well known that the Recent species commonly recorded as C. subglobosa is not identical with the fossil taxon with the same name (Whatley et al. 2003; Smith et al. 2011). The specimens of the exclusively fossil species have been reassigned to the genus Paracypretta as Paracypretta subglobosa (Sowerby, 1840) (Whatley et al. 2003). Klie (1932) showed that the extant species is identical with Cypris granulata Daday, 1898, which therefore becomes the valid name for this extant, circumtropical species (Martens \& Toguebaye 1985).

(5) The genus Candonocypris Sars, 1894 is diagnosed, among others, by a spatulate terminal Mx1-palp segment and two so-called ' $f$ '-setae on the cleaning leg (L7, T3) (Martens 2001a, 2001b; Karanovic 2012). From this, it follows that a number of species commonly assigned to Candonocypris do not belong there: C. deeveyi Tressler, 1954, C. pascheri (Brehm, 1923) and C. pugionis Furtos, 1936 carry one single f-seta on their cleaning leg, while C. fitzroyi McKenzie, 1966 has a cylindrical terminal segment on its Mx1-palp. Candonocypris pugionis fits into the genus Trajancypris and is here transferred to that genus (see below). As no other genus is readily available for the other species, they are provisionally kept in Candonocypris, but are here flagged as needing a new genus assignment.

(6) The genus Cyclocypria Dobbin, 1941 was introduced for the single species Cyclocypria kincaidia Dobbin, 1941, described from the USA. The genus has remained monospecific up to the present. Dobbin Evenson (1969) considered her original description of kincaidia (Dobbin 1941) to apply to a "late instar of Cyclocypris kincaidia", thus implicitly introducing the synonymy of Cyclocypria with Cyclocypris Brady \& Norman, 1889. This view is followed here.

(7) Cypria ophtalmica (Jurine, 1820) and Cypria lacustris Lilljeborg, 1890:

(a) When describing Cypria lacustris, Sars (1890) mentioned that he relied on the description of the species in an unpublished manuscript by Lilljeborg. We here follow Kempf (1980a-d) who lists the species with Lilljeborg as being the author: Cypria lacustris Lilljeborg, 1890 (in Sars 1890).

(b) Cypria lacustris is usually considered an intraspecific form, without a formal taxonomic status of its own, of Cypria ophtalmica because of the occurrence of animals with transitional characters, mainly seen in the number and structure of the female genital processes (Meisch 2000: 217). From the examination, in recent years, of a very large number of individuals belonging to both 'forms' from various regions in Europe, one of us (CM) could not confirm the occurrence of 'transitional' 
animals. Furthermore, the ecology differs: Cypria lacustris almost exclusively colonizes springs, waters connected to springs and the profundal zone of lakes, while Cypria ophtalmica occurs in ponds and the littoral zone of lakes. The rare 'transitional' individuals recorded in the literature might be the result of very occasional hybridisation between two separate species, though this needs to be confirmed, preferably with molecular methods. In conclusion, Cypria lacustris is listed as a valid species in the present list.

(8) Cypria sywulai Meisch, 2000 (emendation of name): This species was originally described as Cypria ophtalmica var. lata Sywula, 1981. The variety was subsequently elevated to species rank as Cypria lata Meisch \& Forró, 1998. When the latter name was shown to be preoccupied by Cypria lata (Dubowsky, 1929) by Kovalenko (1987) (= Cypria curvifurcata var. lata Dubowsky, 1929), Sywula's species was renamed as Cypria sywulae Meisch, 2000, in honour of the Polish ostracodologist Tadeusz Sywula. The ending in 'ae', however, is incorrect and therefore the specific name is here corrected as Cypria sywulai Meisch, 2000, in compliance with article 31.1.2 of the ICZN (the ending in 'ae' is for a woman, 'i' for a man).

(9) Martens (2001) revised the taxonomy of the subfamily Herpetocypridinae Kaufmann, 1900, and placed the genera Parastenocypris Hartmann, 1964 and Gesa Hartmann, 1964 into the synonymy of the genus Chrissia Hartmann, 1957. Here, we also assign species of Parastenocypris and Gesa to Chrissia for which the 'new combination' was not expressly designated.

(10) The species of Neocypridopsis Klie, 1940, a genus originally placed in the subfamily Cypridopsinae of Cyprididae, lack the pincer organ on their cleaning legs (L7/T3), a structure that characterises the entire family. Neocypridopsis therefore most probably does not belong there. The phylogenetic position of Neocypridopsis needs to be clarified through detailed morphological and genetical analyses. Meanwhile, the genus is here provisionally kept in Cypridopsinae.

(11) Bradleytriebella tuberculata (Hartmann, 1964): Strandesia tuberculata Hartmann, 1964, originally described from India, is a junior homonym of Strandesia tuberculata (Sharpe, 1908) (Hartmann \& Petersen 1985), the latter so far only recorded from the USA. Consequently, Hartmann \& Petersen (1985) renamed the species of Hartmann (1964) as Strandesia antetuberculata Hartmann \& Petersen, 1985 nom. nov. More recently, Savatenalinton \& Martens (2009a) transferred Hartmann's species into the newly erected genus Bradleytriebella and assigned it the status of type species of the new genus. The species is listed here with its original specific name and the current generic assignment as Bradleytriebella tuberculata (Hartmann, 1964), with Strandesia antetuberculata Hartmann \& Petersen, 1985 as a synonym.

(12) Eucypris Vávra, 1891 and Candocyprinotus Delorme, 1970: Smith \& Horne (2016) mention Candocyprinotus Delorme, 1970 as a possible junior synonym of Eucypris. As this synonymy needs to be further investigated, Candocyprinotus, with one single species, is maintained here as a separate genus.

(13) Heterocypris congenera (Vávra, 1897) and Hemicypris congenera (Vávra, 1897) sensu Daday 1910 nec Vávra 1897: Martens (1984) recognized that the redescription of Cyprinotus congener Vávra, 1897 provided by Daday (1910) actually applies to a different and still undescribed species, which he provisionally listed as Hemicypris congenera (Daday, 1910). The latter name, however, is not valid in the sense of the ICZN and therefore Daday's (1910) species needs to be formally redescribed with the status of a 'new' species. As this is beyond the scope of the present work, the species of Daday (1910) is listed as mentioned in the paragraph title above. 
Table 1 (continued on next page). Taxonomic changes. New combinations, new names, emendation of name and new synonymies.

\section{New combinations}

1. Fabaeformiscandona alchichica (Cohuo et al., 2017) comb. nov.

Candona alchichica Cohuo et al., 2017

2. Fabaeformiscandona thienemanni (Klie, 1932) comb. nov.

Candona thienemanni Klie, 1932

3. Heterocypris chetumalensis (Yoo et al., 2017) comb. nov.

Manuelcypris chetumalensis Yoo et al., 2017

4. Heterocypris cisternina (Furtos, 1936) comb. nov.

Eucypris cisternina Furtos, 1936

5. Heterocypris tabascena (Yoo et al., 2017) comb. nov.

Manuelcypris tabascena Yoo et al., 2017

6. Physocypria ivanae (Díaz \& Lopretto, 2011) comb. nov.

Keysercypria ivanae Díaz \& Lopretto, 2011

7. Physocypria pelagica (Brehm, 1932) comb. nov.

Cypria pelagica Brehm, 1932

8. Plesiocypridopsis brachychaeta (Goins, 1972) comb. nov.

Potamocypris brachychaeta Goins, 1972

9. Plesiocypridopsis maduraiensis (Victor \& Michael, 1975) comb. nov.

Cypridopsis maduraiensis Victor \& Michael, 1975

10. Pseudocandona arcuata (Klie, 1932) comb. nov.

Candona arcuata Klie, 1932

11. Pseudocandona choi (Karanovic \& Lee, 2012) comb. nov.

Typhlocypris choi Karanovic \& Lee, 2012

12. Pseudocandona pedropalensis (Méhes, 1914) comb. nov.

Candona pedropalensis Méhes, 1914

13. Pseudocandona vasconica (Margalef, 1946) comb. nov.

Candona vasconica (Margalef, 1946)

14. Strandesia gibbosa (Baird, 1838) comb. nov.

Sataracypris gibbosa (Baird, 1838)

15. Strandesia longiforma (Dobbin, 1941) comb. nov.

Prionocypris longiforma Dobbin, 1941

16. Tonnacypris strigata (O.F. Müller, 1776) comb. nov.

Cypris strigata O.F. Müller, 1776

17. Trajancypris pugionis (Furtos, 1936) comb. nov.

Candonocypris pugionis Furtos, 1936

\section{New names}

1. Candona galinae nom. nov.

Junior homonym: Candona rara Mazepova, 1990

2. Candona maloemorensis nom. nov.

Junior homonym: Candona intermedia Bronstein, 1947

3. Candona mazepovae nom. nov.

Junior homonym: Candona procera Mazepova, 1982

4. Cyclocypris klughi nom. nov.

Junior homonym: Cyclocypris castanea Klugh, 1923

5. Strandesia hulkopensis nom. nov.

Junior homonym: Strandesia hartmanni Mannikeri \& Vaidya, 1990 
Table 1 (continued).

\section{Emendation of name}

1. Cypria sywulai Meisch, 2000

Cypria sywulae Meisch, 2000

\section{New synonymies}

1. Trapezicandonini Karanovic, 2007

Mixtacandonini Karanovic \& Lee, 2012 syn. nov.

2. Baicalocandona Mazepova, 1976 (partim), Candona Baird, 1845 (partim)

Mazepovacandona Karanovic \& Sitnikova, 2017 syn. nov.

3. Cypria Zenker, 1854 (partim), Physocypria Vávra, 1897 (partim)

Keysercypria Karanovic, 2011 syn. nov.

4. Heterocypris Claus, 1892

Manuelcypris Yoo et al., 2017 syn. nov.

5. Strandesia Stuhlmann, 1888

Sataracypris Deb, 1983 syn. nov.

6. Bradleytriebella tuberculata (Hartmann, 1964) Savatenalinton \& Martens 2009a

Strandesia antetuberculata Hartmann \& Petersen, 1985 syn. nov.

7. Cypris pubera O.F. Müller, 1776

Cypris pubera anacantha Daday, 1900 syn. nov.

Cypris pubera diacantha Daday, 1900 syn. nov.

Cypris pubera monacantha Daday, 1900 syn. nov.

Cypris pubera polyacantha Daday, 1892 syn. nov.

Cypris pubera triacantha Daday, 1900 syn. nov.

8. Eucypris virens (Jurine, 1820)

Eucypris obtusa G.W. Müller, 1900 syn. nov.

Eucypris helocrenica Fuhrmann \& Goth, 2011 syn. nov.

9. Herpetocypris chevreuxi (Sars, 1896)

Herpetocypris romei Anichini, 1967 syn. nov.

10. Physocypria pelagica (Brehm, 1932) comb. nov.

Cypria petenensis Ferguson et al., 1964 non Klie, 1932 syn. nov.

11. Pseudocandona insculpta (G.W. Müller, 1900)

Pseudocandona vasconica (Margalef, 1953) syn. nov.

(14) Physocypria koenikei (Daday, 1910) sensu Martens 1982: both the Zenker's organ, with numerous whorls of spines, and the hemipenis of this species appear similar to those seen in the Cyprididae rather than the Cyclocypridinae of the Candonidae, to which the genus Physocypria belongs (Karanovic 2011). However, as no cypridid genus is readily available, the species is here provisionally kept in Physocypria but is flagged as needing a new generic assignment.

(15) Riocypris Klie, 1935: Riocypris was erected for the single species $R$. uruguayensis Klie, 1935, described from Uruguay. The genus remained monospecific until Karanovic (2008) described $R$. hinzeae from Australia, additionally transferring the following two species to Riocypris: R. fitzroyi (McKenzie, 1966), formerly assigned to Candonocypris, and $R$. fontana (Graf, 1931), previously allocated to Eucypris. More recently, Díaz \& Martens (2014) transferred E. fontana into the newly erected genus Argentocypris Díaz \& Martens, 2014. For reasons of both the differences in the structure of the carapace and biogeographic occurrence, the latter authors (op. cit.) expressed their doubts whether the West 
Table 2. Numbers of non-marine species/subspecies and percentages of total number of species in superfamilies and families. *indicates primarily marine families with non-marine representatives.

\begin{tabular}{|c|c|c|c|c|c|}
\hline Superfamilies & $\begin{array}{l}\text { No. of } \\
\text { species }\end{array}$ & $\begin{array}{c}\% \text { of total no. } \\
\text { of species }\end{array}$ & Families & $\begin{array}{l}\text { No. of } \\
\text { species }\end{array}$ & $\begin{array}{c}\% \text { of tota } \\
\text { no. of } \\
\text { species }\end{array}$ \\
\hline \multirow{4}{*}{ Cypridoidea } & \multirow{4}{*}{1760} & \multirow{4}{*}{$75.5 \%$} & Cyprididae & 1006 & $43.2 \%$ \\
\hline & & & Candonidae & 675 & $29.0 \%$ \\
\hline & & & Ilyocyprididae & 41 & $1.8 \%$ \\
\hline & & & Notodromadidae & 38 & $1.6 \%$ \\
\hline \multirow{11}{*}{ Cytheroidea } & \multirow{11}{*}{531} & \multirow{11}{*}{$22.8 \%$} & Entocytheridae & 212 & $9.1 \%$ \\
\hline & & & Limnocytheridae & 163 & $7.0 \%$ \\
\hline & & & ${ }^{*}$ Cytherideidae & 95 & $4.1 \%$ \\
\hline & & & *Leptocytheridae & 23 & $1.0 \%$ \\
\hline & & & *Cytheridae & 13 & $0.6 \%$ \\
\hline & & & *Loxoconchidae & 10 & $0.4 \%$ \\
\hline & & & *Xestoleberididae & 5 & $0.2 \%$ \\
\hline & & & *Cytheruridae & 4 & $0.2 \%$ \\
\hline & & & *Paradoxostomatidae & 2 & $0.1 \%$ \\
\hline & & & *Hemicytheridae & 2 & $0.1 \%$ \\
\hline & & & Kliellidae & 2 & $0.1 \%$ \\
\hline Darwinuloidea & 35 & $1.5 \%$ & Darwinulidae & 35 & $1.5 \%$ \\
\hline Terrestricytheroidea & 4 & $0.2 \%$ & Terrestricytheridae & 4 & $0.2 \%$ \\
\hline
\end{tabular}

Australian R. hinzeae and the South American R. uruguayensis would be congeneric. We follow Díaz \& Martens (2014) and only provisionally keep R. hinzeae in the genus Riocypris. As for $R$. fitzroyi, it is here reallocated to Candonocypris.

\section{New combinations and transfers}

(1) Fabaeformiscandona alchichica (Cohuo et al., 2017) comb. nov.

Candona alchichica Cohuo et al., 2017 was assigned to the acuminata group of Candona by Cohuo et al. (2017a), a species group that now ranks within the genus Fabaeformiscandona sensu Danielopol (1973) and Meisch (2000). The species is therefore here transferred in the latter genus.

(2) Fabaeformiscandona thienemanni (Klie, 1932) comb. nov.

In the original description of Candona thienemanni Klie, 1932, illustrations of the carapace in both lateral and dorsal views show that this species belongs to Fabaeformiscandona sensu Meisch (2000), into which the species is here transferred.

(3) Heterocypris chetumalensis (Yoo et al., 2017) comb. nov. and Heterocypris tabascena (Yoo et al., 2017) comb. nov.

With Manuelcypris Yoo et al., 2017 considered herein to be a junior synonym of Heterocypris (see below under New Synonymies for details), these two species are transferred to the genus Heterocypris. 
(4) Heterocypris cisternina (Furtos, 1936) comb. nov.

Yoo et al. (2017) noted that the fifth limb of the type specimens of Eucypris cisternina Furtos, 1936 lacks the so-called 'c'-seta, which characterises the genus Eucypris as diagnosed by Martens (1989) and transferred the species to the newly erected genus Manuelcypris Yoo et al., 2017. In general, E. cisternina rather perfectly fits the diagnosis of the genus Heterocypris, except, however, for the absence of marginal tubercles on the right valve. Considering that these tubercles are known to be poorly developed or even missing in some populations of Heterocypris - see, for instance, Heterocypris salina (Brady, 1968) (Meisch 2000: 355) - the species is here tentatively transferred to the latter genus.

(5) Physocypria ivanae (Díaz \& Lopretto, 2011) comb. nov.

Following the synonymy of Keysercypria with Cypria and Physocypria (see below under New Synonymies for details), Keysercypria ivanae Díaz \& Lopretto, 2011 is here provisionally transferred to Physocypria. However, the original description indicates that this species has tubercles on both valves, so its generic position needs to be revised.

(6) Physocypria pelagica (Brehm, 1932) comb. nov.

There is much confusion surrounding Cypria pelagica Brehm, 1932 - here referred to as Physocypria pelagica (Brehm, 1932) comb. nov. - known from Central America, and Cypria pelagica Klie, 1932, recorded from Indonesia and India. The taxonomic history of both species is briefly described here. Cypria pelagica Brehm, 1932 was rudimentarily described from Lake Petén Itzá in Guatemala, with a more detailed description presented later by the same author (Brehm 1932, 1939). Ferguson et al. (1964) argued that the original description of Cypria pelagica Brehm was insufficient and therefore considered - but incorrectly so - the name of Brehm (1932) a nomen nudum and consequently introduced a new name for the species: Cypria petenensis Ferguson et al., 1964. More recently, upon examining topotype specimens of Cypria pelagica from Lake Petén Itzá, Pérez et al. (2010) and Cohuo et al. (Online Resource 1 of 2017b) emphasized the presence of marginal tubercles on the right valve, which is a character of the genus Physocypria sensu Meisch (2000). The species is therefore here transferred to the latter genus as Physocypria pelagica (Brehm, 1932) comb. nov. Martens \& Savatenalinton (2011) pointed out that Cypria pelagica Brehm, 1932 has priority over its homonym Cypria pelagica Klie, 1932 - originally described as Cypria javana pelagica Klie, 1932 - and consequently introduced a new name for the latter species, Cypria kliei Martens \& Savatenalinton, 2011, which is therefore the valid name of that species.

(7) Plesiocypridopsis brachychaeta (Goins, 1972) comb. nov. and Plesiocypridopsis maduraiensis (Victor \& Michael, 1975) comb. nov.

Potamocypris brachychaeta Goins, 1972, described from Indiana, USA, and Cypridopsis maduraiensis Victor \& Michael, 1975, known from India, were described as having uropodal rami with cylindrical stems, which is a unique character of the genus Plesiocypridopsis (the ramus is triangularly shaped in all other genera of Cypridopsinae; see Meisch 2000). Both species are therefore here transferred to the genus Plesiocypridopsis (Rome, 1965).

(8) Pseudocandona arcuata (Klie, 1932) comb. nov.

Candona arcuata Klie, 1932, described from the island of Java, Indonesia, was provisionally allocated by Klie (1932) to the rostrata group of Candona, a species group now placed within Pseudocandona (sensu Meisch 2000; Namiotko et al. 2014). The presence of three setae (d1, d2 and dp) on the protopodite of the cleaning leg (Klie 1932: fig. 14) confirms that it belongs to Pseudocandona. The species is therefore here transferred to that genus. 
(9) Pseudocandona choi (Karanovic \& Lee, 2012) comb. nov.

Typhlocypris choi Karanovic \& Lee, 2012, which was described from South Korea, belongs to Pseudocandona sensu Meisch (2000), and is here transferred to this genus.

(10) Pseudocandona pedropalensis (Méhes, 1914) comb. nov.

Candona pedropalensis Méhes, 1914 was ranked within the caribbeana species group (Karanovic 2005), which now belongs to Pseudocandona sensu Meisch (2000). The species is here transferred to that genus.

(11) Pseudocandona vasconica (Margalef, 1946) comb. nov.

Candona vasconica (Margalef, 1946), first described as Candona neglecta var. vasconica from northwestern Spain and thus far unrecorded elsewhere, was elevated to species rank by Margalef (1953). Only females were found. The relatively small carapace size (females: $0.91-0.93 \mathrm{~mm}$ ) and the carapace shape in lateral view (Margalef 1946: pl. 2:1) clearly point towards a species of the genus Pseudocandona. The species is transferred to this genus and is also tentatively listed as a synonym of Pseudocandona insculpta (G.W. Müller, 1900) (see below).

(12) Ramotha crassa (Klie, 1929)

One of us (KM) checked old notes and is no longer convinced that Strandesia crassa Klie, 1939 belongs to Bradleystrandesia as in Martens \& Savatenalinton (2011). The species is here moved back to Ramotha as in Martens (1992).

(13) Strandesia gibbosa (Baird, 1838) comb. nov.

The genus Sataracypris Deb, 1983 was erected to include the single species Cypris gibbosa Baird, 1838, which is characterized by the presence of a conspicuous dorsal hump on both valves. Savatenalinton (2015) pointed out that a similar hump is also seen in Strandesia martensi Savatenalinton, 2015 and that both species therefore probably belong to the same lineage within Strandesia s. lat. Sataracypris gibbosa is here tentatively transferred to the genus Strandesia. The presence/absence of the so-called Triebel's loop on the attachment of the uropodal ramus - a character that remains undescribed in S. gibbosa should confirm or invalidate the new combination proposed here.

(14) Strandesia longiforma (Dobbin, 1941) comb. nov.

This species was first described as Prionocypris longiforma Dobbin, 1941. The seminiferous tubules of the males are spirally enrolled in the anterior area of the valves (Dobbin 1941) and the attachment of the uropodal ramus carries a so-called Triebel's loop (Matzke-Karasz et al. 2014). Both characters are diagnostic of the subfamily Cypricercinae of the family Cyprididae. Külköylüoğlu \& Vinyard (2000) recorded the species as Prionocypris (Strandesia) longiforma. The species is here formally, although tentatively, transferred to the genus Strandesia s. lat.

(15) Tonnacypris strigata (O.F. Müller, 1776) comb. nov.

Cypris strigata O.F. Müller, 1776 was recorded under its junior synonym Cypris lutaria Koch, 1838, with various generic assignments, mainly as Eucypris lutaria (Koch, 1838) - now Tonnacypris lutaria - in all major synopses after 1900 (Alm 1915; Sars 1925; Klie 1938a; Bronstein 1947; Meisch 2000). The species is here formally transferred to Tonnacypris as Tonnacypris strigata (O.F. Müller, 1776) comb. nov. This species is an unused older synonym of T. lutaria (Koch, 1838), which, for reasons of nomenclatural stability, we recommend not be revived. It is thus listed here as Tonnacypris lutaria.

(16) Trajancypris pugionis (Furtos, 1936) comb. nov.

This species, which was originally described as Candonocypris pugionis Furtos, 1936, has a cleaning leg with one single f-seta and therefore does not belong to the genus Candonocypris (there are two 
such setae in Candonocypris - see above). Relying on the detailed original description of the species by Furtos (1936b), it is here transferred to the genus Trajancypris Martens, 1989.

\section{New names}

(1) Candona galinae nom. nov.

Candona rara Mazepova, 1990 is a junior homonym of Candona rara G.W. Müller, 1900. The species of Mazepova (1990) is here renamed as Candona galinae nom. nov., the new specific name being derived from that author's first name.

(2) Candona maloemorensis nom. nov.

Candona intermedia Bronstein, 1947, described from Lake Baikal and only known from there, is a junior homonym of Candona intermedia Furtos, 1933, known from North America. The Baikal species is here renamed as Candona maloemorensis nom. nov., the specific name being derived from the Maloe More (Малое Mope in Russian, in English literally the Small Sea), a strait in Lake Baikal where part of the type material was collected and which is of high relevance for the speciation of many Baikalian organisms.

(3) Candona mazepovae nom. nov.

Candona procera Straub, 1952, a fossil species described from Germany (Straub 1952, Tertiary) and Italy (Devoto 1965, Pleistocene) has nomenclatural priority over Candona procera Mazepova, 1982, an extant species described from Lake Baikal. The latter species is renamed here as Candona mazepovae nom. nov.

(4) Cyclocypris klughi nom. nov.

Cyclocypris castanea Klugh, 1923 is a junior homonym of Cyclocypris castanea Brady, 1913. The species of Klugh (1923) is here renamed as Cyclocypris klughi nom. nov.

(5) Strandesia hulkopensis nom. nov.

Strandesia hartmanni Mannikeri \& Vaidya, 1990 is a junior homonym of Strandesia hartmanni Victor et al., 1980, both described from India. The former species is here renamed as Strandesia hulkopensis nom. nov., the specific name being derived from the name of the pond south of the Indian city of Dharwad from which the type material was collected.

\section{New synonymies}

(1) Trapezicandonini Karanovic, 2007 and Mixtacandonini Karanovic \& Lee, 2012

There has been some nomenclatural confusion about the genera Mixtacandona Klie, 1938 and Trapezicandona Schornikov, 1969 and the names of tribes derived from those two genera. The generic name Mixtacandona was proposed conditionally - but with a clear diagnosis - by Klie (1938b) for Candona laisi Klie, 1938, and therefore has priority over Trapezicandona Schornikov, 1969 (Namiotko \& Danielopol 2002). However, according to Article 40.1 of the ICZN, the validity of family-group names, to which tribe names belong, is not affected by the synonymy of type genera. Herein we synonymize the two tribes and conclude that Trapezicandonini Karanovic, 2007 has priority over Mixtacandonini Karanovic \& Lee, 2012.

(2) Baicalocandona Mazepova, 1976, Candona Baird, 1845 and Mazepovacandona Karanovic \& Sitnikova, 2017

The genus Mazepovacandona Karanovic \& Sitnikova, 2017 - type species: Candona directa Bronstein, 1947 - was recently erected for five previously known candonine species endemic to Lake Baikal. For various reasons, including its rather large morphological diversity, the genus is not accepted here. The five species are listed with Mazepova's original assignments in the list of synonyms of each of the 
species in question, and Mazepovacandona is partly synonymized with Baicalocandona and partly with Candona.

(3) Cypria Zenker, 1854, Physocypria Vávra, 1897 and Keysercypria Karanovic, 2011

The species of the genera Cypria Zenker, 1854 and Physocypria Vávra, 1897 were recently rearranged, in parallel with the erection of the genus Keysercypria Karanovic, 2011 for a number of species extracted from Cypria and Physocypria (Karanovic 2011, 2012). This approach uses as defining characters for the two original genera (a) the presence (Physocypria) / absence (Cypria) of the d2seta on the walking leg and (b) the direction the ovary curve, up (Cypria) or down (Physocypria). The usage of these characters, as well as those used for the definition of the genus Keysercypria, produces genera that appear otherwise morphologically very diverse. Also, these characters cannot be seen in both living and fossil specimens. We conclude that there is no good phylogenetic reason at this stage to prefer the soft part characters over the traditional presence (Physocypria) or absence (Cypria) of marginal tubercles on the right valve, which is easily observable in both living and fossil specimens. A conservative approach is therefore adopted here, which follows the definitions of the genera Cypria and Physocypria as presented, for instance, by Meisch (2000) and we treat Keysercypria partly as a junior synonym of Cypria and partly as a junior synonym of Physocypria. Species that were allocated to Keysercypria by Karanovic (2011) are listed under their previous combinations (see also above).

(4) Heterocypris Claus, 1892 and Manuelcypris Yoo et al., 2017

The diagnosis of Manuelcypris Yoo et al., 2017 is broad, and species have been assigned to this genus inconsistently. For instance, both Heterocypris punctata Keyser, 1975 and Heterocypris antillensis Broodbakker, 1982, transferred to Manuelcypris by Yoo et al. (2017), have setae on the first segment (protopodite) of the sixth limb (Broodbakker 1982: 220, 223) and therefore do not fit the diagnosis of Manuelcypris. Other species in the genus appear to have little to unite them morphologically and the molecular analysis of Yoo et al. (2017) does not at all support the validity of the genus. In consequence, the genus is not used here, and we consider it a synonym of Heterocypris. Existing species are returned to their previous genus, Heterocypris, and the new species in Yoo et al. (2017) are here transferred from Manuelcypris to Heterocypris (see New combinations and transfers section for more details).

(5) Strandesia Stuhlmann, 1888 and Sataracypris Deb, 1983

The type species of the monospecific genus Sataracypris Deb, 1983, Sataracypris gibbosa (Baird, 1838), is tentatively transferred to the genus Strandesia Stuhlmann, 1888 (see above for details). Sataracypris therefore becomes a junior synonym of Strandesia.

(6) Cypris pubera O.F. Müller, 1776

Daday $(1892,1900)$ described the following five varieties of Cypris pubera, mainly in relation to the presence/absence and development of posteroventral marginal spines of the right valve: anacantha (absence of spines), monacantha (one single spine), diacantha (two spines), triacantha (three spines) and polyacantha (more than three spines). We consider these varieties - which were not retained in any of the subsequent faunistic synopses (e.g., G.W. Müller 1912; Klie 1938a; Farkas 1958; Henderson 1990; Meisch 2000) - to express the morphological variability of the species and they are therefore listed here as synonyms of the main form. Daday (1900) does not mention the variety triaculeata of C. pubera, which he had described eight years earlier (Daday 1892). The latter variety was raised to species rank by Fuhrmann \& Pietrzeniuk (1990), mainly based on the difference in the posteroventral spines of the right valve. However, we here consider triarticulata to rank within the variability range of Cypris pubera, a variation that is indeed to be expected in a species that reproduces almost entirely parthenogenetically (males of C. pubera are so far only known from one site in Turkey; Schäfer 1952). Cypris triaculeata sensu Fuhrmann \& Pietrzeniuk 1990 is therefore listed as a synonym of Cypris pubera. 
MEISCH C. et al., Global checklist of extant non-marine Ostracoda

(7) Eucypris virens (Jurine, 1820), Eucypris obtusa Fuhrmann \& Goth, 2011 and Eucypris helocrenica Fuhrmann \& Goth, 2011

Eucypris virens is known to be highly variable in carapace shape (G.W. Müller 1900; Tétart 1985; Meisch 2000) and to consist of a complex of at least 40 and potentially as many as 60 or more so-called cryptic, morphologically unrecognizable 'genetic species' (Bode et al. 2010; Koenders et al. 2012). For that reason and awaiting further morphological and genetical studies, the following two species, which differ from $E$. virens only in small differences in the shape of the carapace and valves, are placed in the list of synonyms of E. virens: Eucypris obtusa Fuhrmann \& Goth, 2011 (syn.: Eucypris virens var. obtusa G.W. Müller, 1900) and E. helocrenica Fuhrmann \& Goth, 2011.

(8) Herpetocypris chevreuxi (Sars, 1896) and Herpetocypris romei Anichini, 1967

Taking into account the morphological variation that is to be expected in a species that reproduces parthenogenetically (males of $H$. chevreuxi have so far never been found), the detailed original description of $H$. romei rather perfectly complies with the description of Herpetocypris chevreuxi. $H$. romei is therefore put in synonymy with $H$. chevreuxi.

(9) Mixtacandona talianae Gliozzi \& Mazzini, 1998 and Mixtacandona italica Karanovic \& Pesce, 2001 The possible synonymy of these species has previously been suggested by Krstić (2006) and Mazzini et al. (2017). Upon comparing the detailed descriptions of both species, the synonymy is here confirmed.

(10) Physocypria pelagica (Brehm, 1932) comb. nov. and Cypria petenensis Ferguson et al., 1964 syn. nov. non Klie 1932

As noted above, the replacement name Cypria petenensis Ferguson et al., 1964 for Physocypria pelagica (Brehm, 1932) by Ferguson et al. (1964) was unnecessary, and so herein it is considered to be a junior synonym. See section above under Physocypria pelagica (Brehm, 1932) comb. nov. for more details.

(11) Pseudocandona insculpta (G.W. Müller, 1900) and Pseudocandona vasconica (Margalef, 1946) The original description of Candona neglecta var. vasconica mentions a small distal "appendage" on the anterior side of the penultimate A2 segment, which obviously is the reduced G2-claw that characterizes the female antennae of Ps. insculpta and Ps. compressa. The shallow valve pits reported by Margalef (1946) tend to indicate that he either dealt with adults of Ps. insculpta or with late juveniles of PS. compressa. The species is here transferred to Pseudocandona (see above) and tentatively listed as a synonym of Pseudocandona insculpta (G.W. Müller, 1900).

\section{Uncertain generic assignments}

As already pointed out by Karanovic (2012: 476), the following three species assigned to Eucypris do not belong there: E. areguensis Daday, 1905, E. trapezoides Hartmann, 1962 and E. afghanistanensis Hartmann, 1964. Indeed, the posterior seta of the uropodal ramus appears claw-like and stands close to the terminal claws, a character that excludes these species from Eucypris and is diagnostic for part of the species of the subfamily Herpetocypridinae. However, as this demands a detailed taxonomic analysis, the three species are provisionally listed under Eucypris.

\section{Uncertain species}

Candona detecta (O.F. Müller, 1776) Baird, 1845. The original description of this species is very poor and lacks illustrations. In his redescription of the species, the same author (O.F. Müller 1785) mentions and illustrates a uropodal ramus with a posterior seta.

Daday (1900) considered C. detecta to be identical to Candonopsis kingsleii (Brady \& Robertson, 1870). However, the presence of a posterior uropodal seta precludes this species belonging to the genus Candonopsis Vávra, 1891 (fide Karanovic \& Marmonier 2002). 
G.W. Müller (1912) considered Candona detecta to be identical to Candona protzi Hartwig, 1898, now Fabaeformiscandona protzi (Hartwig, 1898). Sars (1925) pointed out that $C$. detecta may be a juvenile of Candona caudata Kaufmann, 1900, now Fabaeformiscandona caudata (Kaufmann, 1900). The inclusion of C. detecta in the genus Fabaeformiscandona Krstić, 1972 appears sound to us and we therefore propose the formal transfer of the species into that genus: Fabaeformiscandona detecta (O.F. Müller, 1776) comb. nov. Being an uncertain species, because it is presently unrecognizable, F. detecta is listed as such.

\section{Species and genera removed from the main list}

Cythereis rivulorum Rome, 1943, described from a river in Belgium and later transferred to the monospecific genus Romeis Sywula, 1970, was shown to represent a marine species inadvertently introduced into a freshwater sample (Meisch 2000). Both the genus and species are therefore removed from the present world list of non-marine species.

\section{Biogeography}

To date, the PA region, with 799 species, is the most speciose of all regions, followed by the AT with 453 species and the NA with 439 species (Fig. 1). The proportions of families in each region varies considerably, with Cyprididae constituting $74.5 \%$ of species in the OL, $67.8 \%$ in the AT and $56.8 \%$ in the NT, but only $27.3 \%$ and $29.7 \%$ in the NA and PA, respectively. Of the 439 species recorded from the NA, $172(39.2 \%)$ are entocytherids, a family commensal on other crustaceans, typically crayfish. The entocytherids in the NA represent $81.1 \%$ of all species known in this family, and their high diversity in this region mirrors the high diversity of crayfish in North America (Crandall \& Buhay 2008). Two thousand and ninety-two species $(89.8 \%$ ) are currently known from one zoogeographical region only, suggesting a very high rate of endemism at this level. No species have been reported from all regions, while only six, Cypretta turgida (Sars, 1896), Cypridopsis vidua (O.F. Müller, 1776), Heterocypris incongruens (Ramdohr, 1808), Stenocypris major (Baird, 1859), Darwinula stevensoni (Brady \& Robertson, 1870) and Penthesilenula brasiliensis (Pinto \& Kotzian, 1961), have been reported from six or seven regions, and can be considered cosmopolitan.

\section{History of research and recent trends}

The first non-marine ostracods were named and described in 1776 (O.F. Müller 1776), but for the next 11 decades the number of new descriptions remained very low (Fig. 2). There was a notable increase in species descriptions in the 1890 s, and since then 100 species or more have been described per decade. The most productive decade in terms of new species described was the 1960s, with 338 new species, followed by the 1980s (246 species) and the 1930s (238 species) (Fig. 2).

The Cyprididae and Candonidae are the two largest families, totalling 1681 species, with the Candonidae representing $40.2 \%$. For most decades, the number of Candonidae described was below this ratio, with the exception of the $1940 \mathrm{~s}(61.2 \%)$ and the last three decades (44.5 to $78.3 \%)$. The large relative increase in Candonidae research in the 1940s can be attributed to the book by Bronstein (1947) on ostracods from the former USSR. Since 1990, there were proportionally more new Candonidae described compared with before 1990, with the exception of the 1940s, indicating that recent taxonomic research has generally focused more on the Candonidae and less on the Cyprididae. This is probably due to more recent research on ancient lakes and groundwaters, both of which tend to contain high diversities of Candonidae (e.g., Danielopol \& Hartmann 1986; Mazepova 1990; Martens 1994; Karanovic 2007; Smith \& Janz 2008). There was a notable peak in descriptions of entocytherid ostracods during the 1960s and 70s, with $73.1 \%$ ( 155 species) of the entire family being described during this 20 -year period (Fig. 2). From the 1980s onwards, only 16 species have been added. 
In the 20-year period from 1998 to 2017, 377 new species of non-marine ostracods were described (Table 3). Sixty-eight percent of these were from the PA and AU regions combined $(28.6 \%$ and $39.3 \%$ respectively), while over the same period the numbers of new species described from the other regions have been much lower. The NA is notable for the low numbers of new species (10 species) discovered there over the last 20 years, forming only $2.7 \%$ of the global total of new species during this time period and $2.3 \%$ of the region's known fauna. In contrast, new species found in the AU since 1998 form $45.1 \%$ of the entire known non-marine ostracod fauna from that region, while for the PA the figure is $13.6 \%$. Whether this reflects a true difference in underlying diversity of the NA compared with other regions or is a result of diminishing research efforts in the NA over the last 20 years is currently unclear.

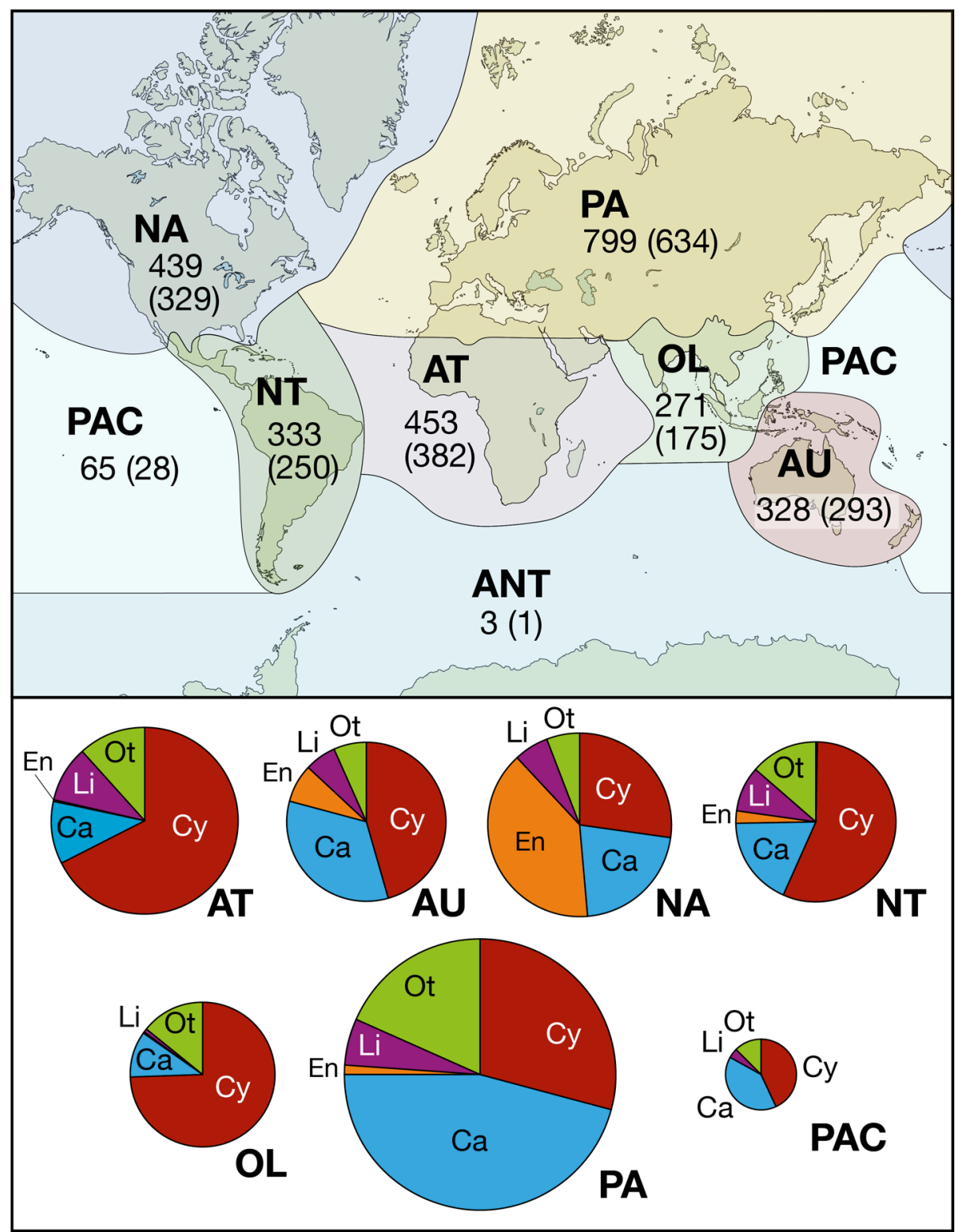

Fig. 1. Numbers of non-marine ostracod species reported in each zoogeographical region, endemic species in parentheses, and proportions of species in non-marine families in each region. Abbreviations: $\mathrm{Cy}=$ Cyprididae; $\mathrm{Ca}=$ Candonidae $\mathrm{En}=$ Entocytheridae $\mathrm{Li}=$ Limnocytheridae; $\mathrm{Ot}=$ other families . Sizes of pie charts are proportional to the number of species reported from each region. 
Table 3. Numbers of new species described in the 20-year period between 1998 and 2017 in the different zoogeographical regions.

\begin{tabular}{cccc}
\hline Region & $\begin{array}{c}\text { New species described } \\
\mathbf{1 9 9 8} \text { to 2017 }\end{array}$ & $\begin{array}{c}\text { \% of total of each } \\
\text { region }\end{array}$ & $\begin{array}{c}\text { \% of total of new } \\
\text { species 1998 to 2017 }\end{array}$ \\
\hline AT & 31 & 6.8 & 8.2 \\
AU & 148 & 45.1 & 39.3 \\
NA & 10 & 2.3 & 2.7 \\
NT & 40 & 12.2 & 10.6 \\
OL & 32 & 11.8 & 8.5 \\
PA & 108 & 13.6 & 28.6 \\
PAC & 8 & 12.3 & 2.1 \\
Total & 377 & & 100.0 \\
\hline
\end{tabular}

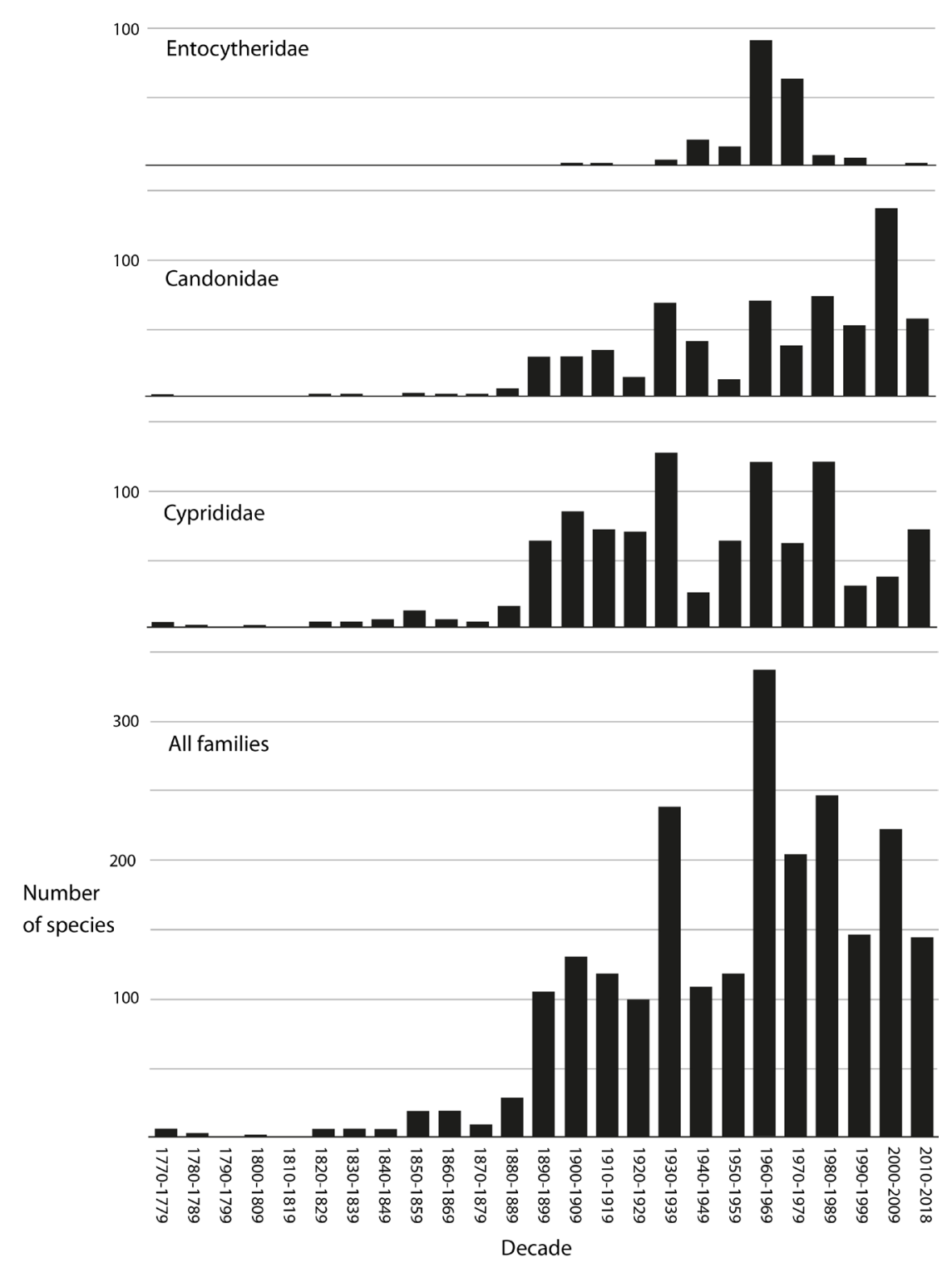

Fig. 2. Numbers of new species of non-marine ostracods described per decade for all families and the three largest families. 
The hierarchical taxonomic position of the extant genera of non-marine Ostracoda

Class Ostracoda Latreille, 1802

Subclass Podocopa Sars, 1866

Order Podocopida Sars, 1866

Suborder Cypridocopina Baird, 1845

Superfamily Cypridoidea Baird, 1845

Family Cyprididae Baird, 1845

Subfamily Batucyprettinae Victor \& Fernando, 1981

Batucypretta Victor \& Fernando, 1981

Subfamily Bennelongiinae Martens et al., 2012

Bennelongia De Deckker \& McKenzie, 1981

Subfamily Bradycypridinae Hartmann \& Puri, 1974

Bradycypris Sars, 1925

Paracypretta Sars, 1924

Zonocypretta De Deckker, 1981

Subfamily Callistocypridinae Schornikov, 1980

Callistocypris Schornikov, 1980

Subfamily Cyprettinae Hartmann, 1971

Cypretta Vávra, 1895

Pseudocypretta Klie, 1932

Subfamily Cypricercinae McKenzie, 1971

Tribe Bradleystrandesiini Savatenalinton \& Martens, 2009

Bradleystrandesia Broodbakker, 1983

Bradleytriebella Savatenalinton \& Martens, 2009

Spirocypris Sharpe, 1903

Tribe Cypricercini McKenzie, 1971

Bradleycypris McKenzie, 1982

Cypricercus Sars, 1895

Pseudostrandesia Savatenalinton \& Martens, 2009

Strandesia Stuhlmann, 1888

Tribe Nealecypridini Savatenalinton \& Martens, 2009

Astenocypris G.W. Müller, 1912

Diaphanocypris Würdig \& Pinto, 1990

Nealecypris Savatenalinton \& Martens, 2009

Tanycypris Triebel, 1959

Subfamily Cypridinae Baird, 1845

Alboa De Deckker, 1981

Chlamydotheca Saussure, 1858

Cypris O.F. Müller, 1776

Globocypris Klie, 1939

Mnementh Martens, 2007

Neocypridella Vávra, 1895

Pseudocypris Daday, 1910

Ramotha Martens, 1992

Subfamily Cypridopsinae Kaufmann, 1900

Tribe Cypridopsini Kaufmann, 1900

Austrocypridopsis McKenzie, 1982

Bryocypris Røen, 1956

Cavernocypris Hartmann, 1964 
Cypridopsis Brady, 1867

Kapcypridopsis McKenzie, 1977

Klieopsis Martens et al., 1991

Martenscypridopsis Karanovic \& Pesce, 2000

Neocypridopsis Klie, 1940

Plesiocypridopsis Rome, 1965

Pseudocypridopsis Karanovic, 1999

Sarscypridopsis McKenzie, 1977

Siamopsis Savatenalinton, 2017

Tanganyikacypridopsis Martens, 1985

Thermopsis Külköylüoğlu et al., 2003

Tungucypridopsis Victor, 1983

Tribe Potamocypridini Ghetti \& McKenzie, 1981

Potamocypris Brady, 1870

Tribe Zonocypridini Higuti \& Martens, 2012

Cabelodopsis Higuti \& Martens, 2012

Zonocypris G.W. Müller, 1898

Subfamily Cyprinotinae Bronstein, 1947

Cyprinotus Brady, 1886

Hemicypris Sars, 1903

Heterocypris Claus, 1892

Homocypris Sars, 1924

Riocypris Klie, 1935

Subfamily Diacypridinae McKenzie, 1978

Diacypris Herbst, 1961

Reticypris McKenzie, 1978

Subfamily Dolerocypridinae Triebel, 1961

Dolerocypris Kaufmann, 1900

Subfamily Eucypridinae Bronstein, 1947

Arctocypris Petkovski et al., 2016

Argentocypris Díaz \& Martens, 2014

Candelacypris Baltanás, 2001

Candocyprinotus Delorme, 1970

Eucyprinotus Sywula, 1972

Eucypris Vávra, 1891

Koencypris Meisch, 2000

Prionocypris Brady \& Norman, 1896

Tonnacypris Diebel \& Pietrzeniuk, 1975

Trajancypris Martens, 1989

Subfamily Herpetocyprellinae Bronstein, 1947 Herpetocyprella Daday, 1909

Subfamily Herpetocypridinae Kaufmann, 1900

Tribe Herpetocypridini Kaufmann, 1900

Candonocypris Sars, 1896

Herpetocypris Brady \& Norman, 1889

Ilyodromus Sars, 1894

Tribe Isocypridini Rome, 1965

Amphibolocypris Rome, 1965

Isocypris G.W. Müller, 1908

Platycypris Herbst, 1957 
Tribe Psychrodromini Martens, 2001

Humphcypris Martens, 1997

Paranacypris Higuti et al., 2009

Psychrodromus Danielopol \& McKenzie, 1977

Somalicypris Martens, 1997

Tribe Stenocypridini Ferguson, 1964

Acocypris Vávra, 1895

Ampullacypris De Deckker, 1981

Chrissia Hartmann, 1957

Stenocypria G.W. Müller, 1901

Stenocypris Sars, 1889

Subfamily Hungarocypridinae Bronstein, 1947

Hungarocypris Vávra, 1906

Subfamily Limanocypridinae Hartmann \& Puri, 1974

Limanocypris Schornikov, 1961

Subfamily Liocypridinae Martens, 2003

Afrocypris Sars, 1924

Liocypris Sars, 1924

Subfamily Megalocypridinae Rome, 1965

Tribe Megalocypridini Rome, 1965

Apatelecypris Rome, 1965

Eundacypris Martens, 1986

Hypselecypris Rome, 1965

Madagascarcypris Martens, 1986

Megalocypris Sars, 1898

Sclerocypris Sars, 1924

Tribe Tanganyikacypridini De Deckker \& Wouters, 1983

Tanganyikacypris Kiss, 1961

Subfamily Mytilocypridinae De Deckker, 1974

Australocypris De Deckker, 1974

Caboncypris De Deckker, 1982

Lacrimicypris Halse \& McRae, 2004

Mytilocypris McKenzie, 1966

Repandocypris Halse \& McRae, 2004

Trigonocypris De Deckker, 1976

Subfamily Ngarawinae De Deckker, 1979

Ngarawa De Deckker, 1979

Subfamily Pelocypridinae Triebel, 1962

Pelocypris Klie, 1939

Subfamily Rudjakoviellinae Triebel, 1973

Rudjakoviella Triebel, 1973

Subfamily Scottiinae Bronstein, 1947

Austromesocypris Martens et al., 2004

Mesocypris Daday, 1910

Scottia Brady \& Norman, 1889

Subfamily uncertain

Amphicypris Sars, 1901

Cypriconcha Sars, 1926 


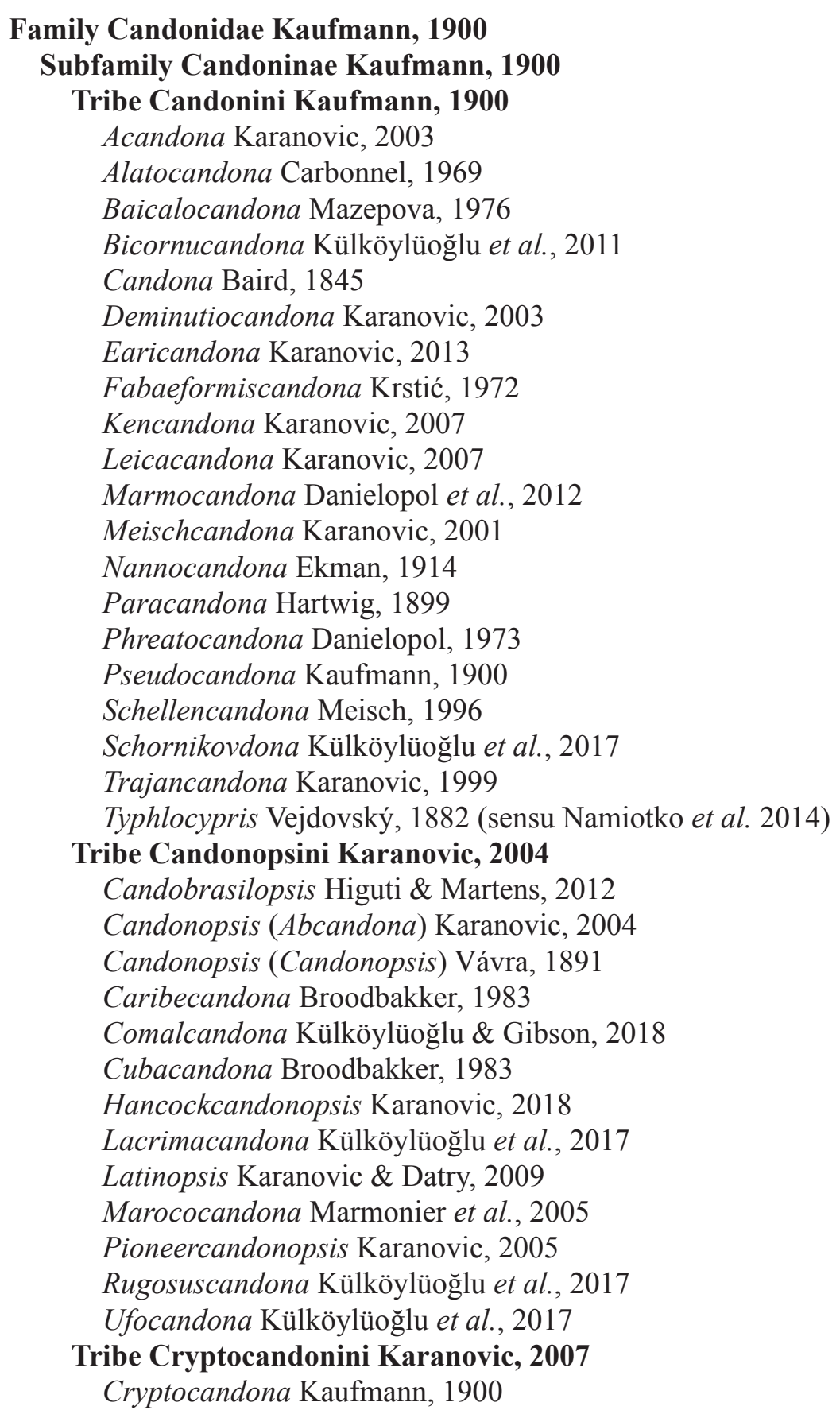

Tribe Humphreyscandonini Karanovic, 2005

Amphitritecandona Karanovic, 2007

Areacandona Karanovic, 2005

Humphreyscandona Karanovic \& Marmonier, 2003

Meridiescandona Karanovic, 2003

Notacandona Karanovic \& Marmonier, 2003

Origocandona Karanovic, 2005

Pierrecandona Karanovic, 2007

Pilbaracandona Karanovic \& Marmonier, 2003

Tribe Namibcypridini Martens, 1992

Danielocandona Broodbakker, 1983

Namibcypris Martens, 1992 
Tribe Terrestricypridini Schornikov, 1980

Caaporacandona Pinto et al., 2005

Terrestricandona Danielopol \& Betsch, 1980

Terrestricypris Schornikov, 1980

Tribe Trapezicandonini Karanovic, 2007

Syn.: Mixtacandonini Karanovic \& Lee, 2012 syn. nov.

Indocandona Gupta, 1984

Mixtacandona Klie, 1938

Undulacandona Smith, 2011

Subfamily Cyclocypridinae Kaufmann, 1900

Allocypria Rome, 1962

Cyclocypris Brady \& Norman, 1889

Cypria Zenker, 1854

Dentocypria Savatenalinton, 2017

Kempfcyclocypris Karanovic, 2011

Mecynocypria Rome, 1962

Namiotkocypria Külköylüoğlu, 2018

Physocypria Vávra, 1897

Subfamily Paracypridinae Sars, 1923

Tribe Renaudcypridini McKenzie, 1980

Renaudcypris McKenzie, 1980

Tribe Thalassocypridini Hartmann \& Puri, 1974

Dolerocypria Tressler, 1937

Hansacypris Wouters, 1984

Mangalocypria Wouters, 1998

Mungava Harding, 1962

Paracypria Sars, 1910

Pontoparta Vávra, 1901

Thalassocypria Hartmann, 1957

Family Ilyocyprididae Kaufmann, 1900

Subfamily Ilyocypridinae Kaufmann, 1900

Ilyocypris Brady \& Norman, 1889

Subfamily Indiacypridinae Hartmann \& Puri, 1974

Indiacypris Hartmann, 1964

Family Notodromadidae Kaufmann, 1900

Subfamily Cyproidinae Hartmann, 1963

Cyprois Zenker, 1854

Subfamily Notodromadinae Kaufmann, 1900

Argentodromas Díaz \& Martens, 2018

Centrocypris Vávra, 1895

Gurayacypris Battish, 1987

Kennethia De Deckker, 1979

Newnhamia King, 1855

Notodromas Lilljeborg, 1853

Subfamily Oncocypridinae De Deckker, 1979

Neozonocypris Klie, 1944

Oncocypris G.W. Müller, 1898

Superfamily Cytheroidea Baird, 1850

Family Cytheridae Baird, 1850

Pericythere Hartmann, 1957

Perissocytheridea Stephenson, 1938 


\section{Family Cytherideidae Sars, 1925}

Subfamily Cytherideinae Sars, 1925

Tribe Cytherideidini Kollmann, 1960

Archeocyprideis Ducasse \& Carbonel, 1994

Cyprideis Jones, 1857

Cytherissa Sars, 1925

Kavalacythereis Wouters, 1979

Mesocyprideis Wouters \& Martens, 1992

Paracyprideis Klie, 1929

Romecytheridea Wouters, 1988

Sarsicytheridea Athersuch, 1982

Tanganyikacythere Ducasse \& Carbonel, 1993

Family Cytheruridae G.W. Müller, 1894

Cytherura Sars, 1866

Semicytherura Wagner, 1957

Family Entocytheridae Hoff, 1942

Subfamily Entocytherinae Hoff, 1942

Ankylocythere Hart, 1962

Ascetocythere Hart, 1962

Aurumcythere Weaver \& Williams, 2017

Cymocythere Hart, 1962

Dactylocythere Hart, 1962

Donnaldsoncythere Rioja, 1942

Entocythere Marshall, 1903

Geocythere Hart, 1962

Harpagocythere Hobbs III, 1965

Hartocythere Hobbs III, 1970

Litocythere Hobbs \& Walton, 1968

Lordocythere Hobbs \& Hobbs, 1970

Okriocythere Hart, 1964

Ornithocythere Hobbs, 1967

Phymocythere Hobbs \& Hart, 1966

Plectocythere Hobbs III, 1965

Psittocythere Hobbs \& Walton, 1975

Rhadinocythere Hart, 1962

Sagittocythere Hart, 1962

Saurocythere Hobbs III, 1969

Thermastrocythere Hobbs \& Walton, 1966

Uncinocythere Hart, 1962

Waltoncythere Hobbs \& Peters, 1978

Subfamily Hartiellinae Danielopol, 1971

Hartiella Danielopol, 1971

Subfamily Microsyssitriinae Hart et al., 1967

Microsyssitria Hart et al., 1967

Subfamily Notocytherinae Hart \& Hart, 1967

Chelocythere Hart \& Hart, 1967

Elachistocythere Hart \& Hart, 1970

Herpetocythere Hart \& Hart, 1967

Hesperocythere Hart \& Hart, 1967

Laccocythere Hart \& Hart, 1971 
Lichnocythere Hart \& Hart, 1967

Notocythere Hart \& Hart, 1967

Riekocythere Hart \& Hart, 1967

Subfamily Sphaeromicolinae Hart, 1962

Hobbsiella Danielopol \& Hart, 1985

Sphaeromicola Paris, 1916

Family Hemicytheridae Puri, 1953

Tyrrhenocythere Ruggieri, 1955

Family Kliellidae Schäfer, 1945

Kliella Schäfer, 1945

Nannokliella Schäfer, 1945

Family Leptocytheridae Sars, 1925

Amnicythere Devoto, 1965

Leptocythere Sars, 1925

Family Limnocytheridae Sars, 1925

Subfamily Limnocytherinae Sars, 1925

Tribe Leucocytherini Danielopol et al., 1990

Athalocythere Schornikov, 1986

Leucocythere Kaufmann, 1900

Leucocytherella Huang, 1982

Ovambocythere Martens, 1989

Potamocythere Schornikov, 1986

Tribe Limnocytherini Klie, 1938

Galolimnocythere Schornikov, 1973

Kiwicythere Martens, 1992

Korannacythere Martens, 1996

Limnocythere Brady, 1868

Limnocytherina Negadaev-Nikonov, 1967

Neolimnocythere Delachaux, 1928

Paracythereis Delachaux, 1928

Paralimnocythere Carbonnel, 1965

Subfamily Timiriaseviinae Mandelstam, 1960

Syn.: Metacypridinae Danielopol, 1960 (fide Colin \& Danielopol 1978)

Afrocythere Klie, 1935

Cytheridella Daday, 1905

Dolekiella Gidó et al., 2007

Elpidium F. Müller, 1880

Frambocythere Colin, 1981

Gomphocythere Sars, 1924

Gomphodella De Deckker, 1981

Intrepidocythere Pinto et al., 2008

Kovalevskiella Klein, 1963

Metacypris Brady \& Robertson, 1870

Family Loxoconchidae Sars, 1925

Cytheromorpha Hirschmann, 1909

Elofsonia Wagner, 1957

Loxoconcha Sars, 1866

Pseudolimnocythere Klie, 1938

Sanyuania Zhao \& Han, 1980 
Family Paradoxostomatidae Brady \& Norman, 1989

Cytherois G.W. Müller, 1884

Family Xestoleberididae Sars, 1928

Xestoleberis Sars, 1866

Superfamily Darwinuloidea Brady \& Robertson, 1885

Family Darwinulidae Brady \& Robertson, 1885

Alicenula Rossetti \& Martens, 1998

Darwinula Brady \& Robertson, 1885

Isabenula Rossetti et al., 2011

Microdarwinula Danielopol, 1969

Penthesilenula Rossetti \& Martens, 1998

Vestalenula Rossetti \& Martens, 1998

Superfamily Terrestricytheroidea Schornikov, 1969

Family Terrestricytheridae Schornikov, 1969

Terrestricythere Schornikov, 1969

\section{Alphabetic checklist of the extant, non-marine Ostracoda}

Acandona Karanovic, 2003

*Acandona admiratio Karanovic, 2003: AU

Acandona memoria Karanovic, 2003: AU

Acocypris acuminata Rome, 1962: AT

Acocypris Vávra, 1895

Acocypris angulosa (Daday, 1910) Martens 1984: AT

Eucypris angulosa Daday, 1910

*Acocypris capillata (Vávra, 1895) G.W. Müller 1898: AT

Cypris capillata Vávra, 1895

Syn.: Parastenocypris unispinosa Onyedineke, 2000 (fide Martens 2001a)

Acocypris hirsuta Rome, 1965: AT

Acocypris hyalina Lowndes, 1931: AT

Acocypris longiuscula Rome, 1965: AT

Acocypris platybasis (Lowndes, 1932) Martens 1984: AT

Stenocypris platybasis Lowndes, 1932

Acocypris stenocyproides Klie, 1938: AT

*Afrocypris barnardi Sars, 1924: AT

Afrocypris Sars, 1924

*Afrocythere rostrata Klie, 1935: AT

Afrocythere Klie, 1935

Alatocandona Carbonnel, 1969

Type species: Alatocandona bronsteini Carbonnel, 1969 (fossil species)

Alatocandona gajewskajae (Bronstein, 1947) Carbonnel 1969: PA

Pseudocandona gajewskajae Bronstein, 1947

*Alboa worooa De Deckker, 1981: AU

Alboa De Deckker, 1981

Alicenula Rossetti \& Martens, 1998

Alicenula furcabdominis (Keyser, 1976) Rossetti \& Martens 1998: NA, NT

Darwinula furcabdominis Keyser, 1976 
MEISCH C. et al., Global checklist of extant non-marine Ostracoda

Alicenula inversa (Martens \& Rossetti, 1997) Rossetti \& Martens 1998: AT

Darwinula inversa Martens \& Rossetti, 1997

*Alicenula serricaudata (Klie, 1935) Rossetti \& Martens 1998: AT, NT, OL

Darwinula serricaudata Klie, 1935

Syn.: Darwinula serricaudata espinosa Pinto \& Kotzian, 1961 (fide Martens \& Rossetti 1997)

Alicenula yucatanensis Macario-González et al., 2018: NT

Allocypria aberrans Rome, 1962: AT

Allocypria Rome, 1962

Allocypria claviformis (Sars, 1910) Rome 1962: AT

Paracypria claviformis Sars, 1910

Allocypria flexuosa (Sars, 1910) Martens 1984: AT

Paracypria flexuosa Sars, 1910

Allocypria humilis (Sars, 1910) Martens 1984: AT

Paracypria humilis Sars, 1910

*Allocypria inclinata Rome, 1962: AT

Allocypria mucronata Rome, 1962: AT

Allocypria navicula Rome, 1962: AT

Allocypria oculata (Kiss, 1959) Martens 1984: AT

Paracypria oculata Kiss, 1959

Allocypria reniformis (Sars, 1910) Martens 1984: AT

Paracypria reniformis Sars, 1910

Amnicythere Devoto, 1965

Syn.: Leptocythere (Amnicythere) Devoto, 1965

Amnicythere Devoto, 1965 in Stancheva 1968

Type species: Leptocythere (Amnicythere) fallax Devoto, 1965 (fossil species)

Amnicythere angulata (Klie, 1939) Namiotko et al. 2012: PA

Leptocythere angulata Klie, 1939

Amnicythere karamani (Klie, 1939) Namiotko et al. 2012: PA

Leptocythere karamani Klie, 1939

Amnicythere prespensis (Petkovski, 1959) Namiotko et al. 2012: PA

Leptocythere prespensis Petkovski, 1959

Amnicythere proboscidea (Klie, 1939) Namiotko et al. 2012: PA

Leptocythere proboscidea Klie, 1939

Amphibolocypris Rome, 1965

Amphibolocypris arida Jocqué \& Martens, 2010: AT

*Amphibolocypris exigua Rome, 1965: AT

Amphicypris Sars, 1901

Amphicypris argentinensis Fontana \& Ballent, 2005: NT

*Amphicypris nobilis Sars, 1901: NA, NT, PA

Syn.: Herpetocypris obliqua Daday, 1902 (fide Martens \& Behen 1994)

Amphicypris pestai (Graf, 1931) Martens \& Behen 1994: NT

Eucypris pestai Graf, 1931

Amphitritecandona Karanovic, 2007

*Amphitritecandona prima Karanovic, 2007: AU

Amphitritecandona secunda Karanovic, 2007: AU 
Ampullacypris De Deckker, 1981

*Ampullacypris oblongata (Sars, 1896) Henry 1923: AU

Cypris oblongata Sars, 1896

Ankylocythere Hart, 1962

Ankylocythere ancyla Crawford, 1965: NA

Ankylocythere barbouri Villalobos \& Hobbs, 1974: NA

Ankylocythere bidentata (Rioja, 1949) Hart 1962: NT

Entocythere bidentata Rioja, 1949

Ankylocythere burkeorum Hobbs III, 1971: NA

Ankylocythere carpenteri Hobbs \& McClure, 1983: NA

Ankylocythere chipola Hobbs III, 1978: NA

Ankylocythere copiosa (Hoff, 1942) Hart 1962: NA

Entocythere copiosa Hoff, 1942

Ankylocythere cubensis (Rioja, 1955) Hobbs 1966: NT

Entocythere heterodonta cubensis Rioja, 1955

Ankylocythere ephydra Hart \& Hart, 1971: NA

Ankylocythere freyi Hobbs III, 1978: NA

Ankylocythere hamata (Hobbs, 1957) Hart 1962: NA

Entocythere hamata Hobbs, 1957

Ankylocythere harmani Hobbs, 1966: NA

*Ankylocythere heterodonta (Rioja, 1940) Hart 1962: NA

Entocythere heterodonta Rioja, 1940

Syn.: Entocythere talirotunda Rioja, 1949 (fide Hobbs 1966)

Ankylocythere hobbsi (Hoff, 1944) Hart 1962: NA

Entocythere hobbsi Hoff, 1944

Ankylocythere hyba Hobbs \& Walton, 1963: NA

Ankylocythere krantzi Hobbs III, 1978: NA

Ankylocythere maya Hobbs, 1971: NA

Ankylocythere prolata Hobbs \& Peters, 1991: NA

Ankylocythere sinuosa (Rioja, 1942) Hart 1962: NA, PA

Entocythere (Cytherites) sinuosa Rioja, 1942

Syn.: Entocythere tiphophila Crawford, 1959 (fide Peters \& Pugh 1999)

Ankylocythere spargosis Andolshek \& Hobbs, 1986: NA

Ankylocythere tallapoosa Hart \& Hart, 1971: NA

Ankylocythere talulus (Hoff, 1944) Hart 1962: NA

Entocythere talulus Hoff, 1944

Ankylocythere toltecae Hobbs, 1971: NA

Ankylocythere tridentata Hart, 1964: NA

Ankylocythere villalobosi Hobbs, 1971: NA

Apatelecypris Rome, 1965

*Apatelecypris schultzei (Daday, 1913) McKenzie 1971: AT

Herpetocypris schultzei Daday, 1913

Syn.: Megalocypris brevis Sars, 1924 (fide Martens 1986)

Archeocyprideis Ducasse \& Carbonel, 1994

*Archeocyprideis tuberculata Ducasse \& Carbonel, 1994: AT

Arctocypris Petkovski et. al., 2016

Arctocypris arctica (Olofsson, 1918) Petkovski et al. 2016: PA

Eucypris arctica Olofsson, 1918 
Arctocypris dulcifons (Diebel \& Pietrzeniuk, 1969) Petkovski et al. 2016: PA

Eucypris dulcifons Diebel \& Pietrzeniuk, 1969

Arctocypris foveata (Delorme, 1968) Petkovski et al. 2016: NA

Eucypris foveata Delorme, 1968

*Arctocypris fuhrmanni Petkovski et al., 2016: PA

Areacandona Karanovic, 2005

Syn.: Neocandona Karanovic, 2005 (fide Karanovic 2007)

Areacandona akatallele Karanovic, 2007: AU

Areacandona ake Karanovic, 2007: AU

Areacandona arteria Karanovic, 2005: AU

Areacandona astrepte Karanovic, 2007: AU

Areacandona atomus Karanovic, 2007: AU

Areacandona bluffi Karanovic, 2007: AU

Areacandona brookanthana Karanovic, 2007: AU

Areacandona cellulosa Karanovic, 2007: AU

Areacandona clementia Karanovic, 2007: AU

Areacandona cognata Karanovic, 2007: AU

Areacandona cylindrata Karanovic, 2007: AU

Areacandona dec Karanovic, 2007: AU

Areacandona fortescueiensis Karanovic, 2007: AU

Areacandona incogitata Karanovic, 2007: AU

Areacandona iuno Karanovic, 2007: AU

Areacandona jessicae Karanovic, 2007: AU

Areacandona korallion Karanovic, 2007: AU

Areacandona krypte Karanovic, 2007: AU

Areacandona lepte Karanovic, 2007: AU

*Areacandona mulgae Karanovic, 2005: AU

Areacandona nammuldi Karanovic, 2007: AU

Areacandona newmani (Karanovic, 2005) Karanovic 2007: AU

Neocandona newmani Karanovic, 2005

Areacandona novitas (Karanovic, 2005) Karanovic 2007: AU

Neocandona novitas Karanovic, 2005

Areacandona quasilepte Karanovic, 2007: AU

Areacandona scanloni Karanovic, 2007: AU

Areacandona stefani Karanovic, 2007: AU

Areacandona triangulum Karanovic, 2007: AU

Areacandona undulata Karanovic, 2007: AU

Areacandona weelumurrae Karanovic, 2007: AU

Areacandona yuleae Karanovic, 2007: AU

Argentocypris Díaz \& Martens, 2014

Argentocypris fontana (Graf, 1931) Díaz \& Martens 2014: ANT, NT

Cypris fontana Graf, 1931

Eucypris fontana (Graf, 1931) De Deckker 1981

*Argentocypris sara Díaz \& Martens, 2014: NT

Argentocypris sarsi (Daday, 1902) Díaz \& Martens 2014: NT

Eucypris sarsii Daday, 1902 
Argentodromas Díaz \& Martens, 2018

*Argentodromas bellanella Díaz \& Martens, 2018: NT

Ascetocythere Hart, 1962

*Ascetocythere asceta (Hobbs \& Walton, 1962) Hart 1962: NA

Entocythere asceta Hobbs \& Walton, 1962

Ascetocythere batchi Hobbs \& Walton, 1968: NA

Ascetocythere bouchardi Hobbs \& Walton, 1975: NA

Ascetocythere coryphodes Hobbs \& Hart, 1966: NA

Ascetocythere cosmeta Hobbs \& Hart, 1966: NA

Ascetocythere didactylata Hobbs \& Hart, 1966: NA

Ascetocythere hoffmani Hobbs \& Hart, 1966: NA

Ascetocythere holti Hobbs \& Walton, 1970: NA

Ascetocythere hyperoche Hobbs \& Hart, 1966: NA

Ascetocythere jezerinaci Hobbs \& McClure, 1983: NA

Ascetocythere lita Hobbs \& Hobbs, 1970: NA

Ascetocythere myxoides Hobbs \& Hart, 1966: NA

Ascetocythere ozalea Hobbs \& Hart, 1966: NA

Ascetocythere pseudolita Hobbs \& Walton, 1975: NA

Ascetocythere riopeli Hobbs \& Walton, 1976: NA

Ascetocythere sclera Hobbs \& Hart, 1966: NA

Ascetocythere stockeri Hobbs \& Peters, 1989: NA

Ascetocythere triangulata Hobbs \& Walton, 1975: NA

Ascetocythere veruta Hobbs \& Walton, 1975: NA

Astenocypris G.W. Müller, 1912

*Astenocypris papyracea (Sars, 1903) G.W. Müller 1912: OL, PA

Leptocypris papyracea Sars, 1903

Athalocythere Schornikov, 1986

*Athalocythere chankensis Schornikov, 1986: PA

Aurumcythere Weaver \& Williams, 2017

*Aurumcythere tillmani Weaver \& Williams, 2017: NA

Australocypris De Deckker, 1974

Australocypris beaumonti Halse \& McRae, 2004: AU

Australocypris benetti Halse \& McRae, 2004: AU

Australocypris dispar De Deckker, 1981: AU

Australocypris hypersalina De Deckker, 1974: AU

Australocypris insularis (Chapman, 1966) De Deckker 1974: AU

Eucypris insularis Chapman, 1966

Australocypris mongerensis Halse \& McRae, 2004: AU

Australocypris rectangularis De Deckker, 1978: AU

*Australocypris robusta De Deckker, 1974: AU

Austrocypridopsis McKenzie, 1982

*Austrocypridopsis terrilli McKenzie, 1982: AU

Austromesocypris Martens et al., 2004

Austromesocypris australiensis (De Deckker, 1983) Martens et al. 2004: AU

Mesocypris australiensis De Deckker, 1983 
MEISCH C. et al., Global checklist of extant non-marine Ostracoda

*Austromesocypris berentsae Martens et al., 2004: AU

Austromesocypris bluffensis Karanovic et al., 2012: AU

Austromesocypris tasmaniensis (De Deckker, 1983) Martens et al. 2004: AU

Mesocypris tasmaniensis De Deckker, 1983

Baicalocandona Mazepova, 1976

Baikalocandona Mazepova, 1976 (misspelling)

Syn.: Mazepovacandona Karanovic \& Sitnikova, 2017 (partim) syn. nov.

Baicalocandona ambagiosa Mazepova, 1976: PA

Baicalocandona aspiranti Mazepova, 1984: PA

*Baicalocandona bivia Mazepova, 1976: PA

Baicalocandona borutskii Mazepova, 1985: PA

Baicalocandona bronsteini Mazepova, 1976: PA

Baicalocandona dorsoconcava Mazepova, 1976: PA

Baicalocandona dorsoconcava dorsoconcava Mazepova, 1976

Baicalocandona dorsoconcava applicata Mazepova, 1976

Baicalocandona dorsoconcava finitima Mazepova, 1976

Baicalocandona dorsoconcava insularis Mazepova, 1984

Baicalocandona navitarum Mazepova, 1976: PA

Mazepovacandona navitarum (Mazepova, 1976) Karanovic \& Sitnikova 2017

Baicalocandona profunda Mazepova, 1976: PA

Baicalocandona ushkani Mazepova, 1984: PA

Baicalocandona zenkevichi Mazepova, 1976: PA

Batucypretta Victor \& Fernando, 1981

*Batucypretta paradoxa Victor \& Fernando, 1981: OL

Bennelongia De Deckker \& McKenzie, 1981

Bennelongia australis (Brady, 1886) De Deckker \& McKenzie 1981: AU

Chlamydotheca australis Brady, 1886

Bennelongia barangaroo De Deckker, 1981: AU

Bennelongia bidgelangensis Martens et al., 2012: AU

Bennelongia calei Martens et al., 2013: AU

Bennelongia coondinerensis Martens et al., 2012: AU

Bennelongia cuensis Martens et al., 2012: AU

Bennelongia cygnus Martens et al., 2012: AU

Bennelongia dedeckkeri Shearn et al., 2012: AU

Bennelongia frumenta Martens et al., 2012: AU

Bennelongia gnamma Martens et al., 2013: AU

Bennelongia gwelupensis Martens et al., 2012: AU

*Bennelongia harpago De Deckker \& McKenzie, 1981: AU

Bennelongia hirsuta Martens et al., 2013: AU

Bennelongia ivanae Martens et al., 2013: AU

Bennelongia kimberleyensis Martens et al., 2012: AU

Bennelongia koendersae Martens et al., 2015: AU

Bennelongia lata Martens et al., 2012: AU

Bennelongia mckenziei Shearn et al., 2012: AU

Bennelongia mcraeae Martens et al., 2013: AU

Bennelongia muggon Martens et al., 2015: AU

Bennelongia nimala De Deckker, 1981: AU

Bennelongia pinderi Martens et al., 2015: AU 
Bennelongia pinpi De Deckker, 1981: AU

Bennelongia regina Shearn et al., 2012: AU

Bennelongia scanloni Martens et al., 2013: AU

Bennelongia shieli Martens et al., 2015: AU

Bennelongia strellyensis Martens et al., 2012: AU

Bennelongia timmsi Martens et al., 2013: AU

Bennelongia tirigie Martens et al., 2015: AU

Bennelongia triangulata Martens et al., 2015: AU

Bennelongia tunta De Deckker, 1982: AU

Bicornucandona Külköylüoğlu et al., 2011

*Bicornucandona fineganensis Külköylüoğlu et al., 2011: NA

Bradleycypris McKenzie, 1982

*Bradleycypris obliqua (Brady, 1868) McKenzie 1982a: NA, PA

Cypris obliqua Brady, 1868

Syn.: Strandesia clorocelis Anichini, 1967 (fide Pieri et al. 2015)

Syn.: Strandesia mulargiae Anichini, 1967 (fide Pieri et al. 2015)

Syn.: Cypricercus alacer Meisch, 1979 (fide Meisch 2000)

Bradleycypris vittata (Sars, 1903) Savatenalinton \& Martens 2009a: AU, OL, PA

Cypris vittata Sars, 1903

Bradleystrandesia Broodbakker, 1983

Bradleystrandesia columbiensis (Dobbin, 1941) Martens \& Savatenalinton 2011: NA Cypricercus columbiensis Dobbin, 1941

Bradleystrandesia dani (George \& Martens, 1993) Savatenalinton \& Martens 2009b: OL Strandesia dani George \& Martens, 1993

Bradleystrandesia deltoidea (Delorme, 1970) Savatenalinton \& Martens 2009a: NA Cypricercus deltoidea Delorme, 1970

Bradleystrandesia dentifera (Dobbin, 1941) Martens \& Savatenalinton 2011: NA Cypricercus dentifera Dobbin, 1941

Syn.: Cypricercus elongata Dobbin, 1941 (late juvenile instar, fide Dobbin Evenson 1969)

* Bradleystrandesia fuscata (Jurine, 1820) Broodbakker 1983b: NA, NT, PA

Monoculus fuscatus Jurine, 1820

Syn.: Cypris adusta Koch, 1837 (fide Klie 1938a)

Syn.: Cypris fuscata var. major G.W. Müller, 1900 (fide Meisch 2000)

Syn.: Cypris burlingtonensis Turner, 1894 (fide Hoff 1942)

Bradleystrandesia gigantica (Furtos, 1933) Savatenalinton \& Martens 2009a: NA

Eucypris fuscata var. gigantica Furtos, 1933

Eucypris gigantica (Furtos, 1933) Hoff 1942

Bradleystrandesia hirsuta (Fischer, 1851) Martens 1994: NA, PA

Cypris hirsuta Fischer, 1851

Bradleystrandesia mollis (Furtos, 1936) Martens \& Savatenalinton 2011: NA

Cypricercus mollis Furtos, 1936

Bradleystrandesia parva (Hartmann, 1964) Savatenalinton \& Martens 2009b: AT, OL, PA

Strandesia parva Hartmann, 1964

Bradleystrandesia reticulata (Zaddach, 1844) Wouters 1989: NA, OL, PA

Cypris reticulata Zaddach, 1844

Syn.: Bradleystrandesia affinis (Zaddach, 1844) sensu Fuhrmann 2012

Syn.: Cypris affinis Fischer, 1851 (fide Hoff 1942)

Syn.: Cypris testudinaria Sharpe, 1897 (fide Hoff 1942)

Syn.: Cypris fuscata var. minor G.W. Müller, 1900 (fide Meisch 2000) 
MEISCH C. et al., Global checklist of extant non-marine Ostracoda

Bradleystrandesia salina (De Deckker, 1981) Savatenalinton \& Martens 2009b: AU

Cypricercus salinus De Deckker, 1981

Bradleystrandesia serrata (Tressler, 1950) Martens \& Savatenalinton 2011: NA

Cypricercus serrata Tressler, 1950

Bradleystrandesia splendida (Furtos, 1933) Savatenalinton \& Martens 2009a: NA

Cypricercus splendida Furtos, 1933

Bradleystrandesia tincta (Furtos, 1933) Schön \& Martens 1998: NA

Cypricercus tincta Furtos, 1933

Bradleystrandesia tressleri (Ferguson, 1954) Martens \& Savatenalinton 2011: NA

Cypricercus tressleri Ferguson, 1954

Bradleystrandesia trichurensis (Victor et al., 1980) Savatenalinton \& Martens 2009b: OL

Strandesia trichurensis Victor et al., 1980

Bradleystrandesia umbonata (Victor \& Fernando, 1981) Savatenalinton \& Martens 2009b: OL

Strandesia umbonata Victor \& Fernando, 1981

Bradleystrandesia viridis (Chen, 1956) Martens \& Savatenalinton 2011: PA

Strandesia viridis Chen, 1956

Bradleystrandesia weberi (Moniez, 1892) Savatenalinton \& Martens 2009b: OL

Cypris weberi Moniez, 1892

Syn.: Strandesia spinifera Hartmann, 1964 (fide Savatenalinton \& Suttajit 2016)

Bradleytriebella Savatenalinton \& Martens, 2009

Bradleytriebella decorata (Sars, 1903) Savatenalinton \& Martens 2009a: OL, PA

Cypris decorata Sars, 1903

Bradleytriebella lineata (Victor \& Fernando, 1981) Savatenalinton \& Martens 2009a: AT, NT, OL, PA Strandesia lineata Victor \& Fernando, 1981

Bradleystrandesia lineata (Victor \& Fernando, 1981) Savatenalinton \& Martens 2009b

Syn.: Paracypretta amati Martens, 1984 (fide Savatenalinton \& Martens 2010)

Syn.: Strandesia biwaensis Okubo, 2004 (nom. nud., fide Smith et al. 2011)

Bradleytriebella trispinosa (Pinto \& Purper, 1965) Savatenalinton \& Martens 2009a: NT

Cyprinotus trispinosus trispinosus Pinto \& Purper, 1965

Bradleystrandesia trispinosa (Pinto \& Purper, 1965) Savatenalinton \& Martens 2009b

Syn.: Strandesia trispinosa galantis Broodbakker, 1983 (fide Savatenalinton \& Martens 2009b)

*Bradleytriebella tuberculata (Hartmann, 1964) Savatenalinton \& Martens 2009a: OL, PA

Bradleystrandesia tuberculata (Hartmann, 1964) Savatenalinton \& Martens 2009b

Syn.: Strandesia antetuberculata Hartmann \& Petersen, 1985 syn. nov.

Nom. nov. pro Strandesia tuberculata Hartmann, 1964 nec Sharpe, 1908

Bradycypris Sars, 1924

*Bradycypris intumescens (Brady, 1907) Sars 1924a: AT

Cypris intumescens Brady, 1907

Syn.: Cypris radiata G.W. Müller, 1908 (fide Martens 2001b)

Bradycypris strigata Klie, 1939: AT

Bryocypris Røen, 1956

*Bryocypris grandipes Røen, 1956: AT

Caaporacandona Pinto et al., 2005

Caaporacandona iguassuensis Pinto et al., 2005: NT

*Caaporacandona shornikovi Pinto et al., 2005: NT

Cabelodopsis Higuti \& Martens, 2012

*Cabelodopsis hispida (Sars, 1901) Higuti \& Martens 2012a: NT

Cypridopsella hispida Sars, 1901 
Caboncypris De Deckker, 1982

Caboncypris kondininensis Halse \& McRae, 2004: AU

*Caboncypris nunkeri De Deckker, 1982: AU

Callistocypris Schornikov, 1980

Callistocypris mckenziei Pinto et al., 2005: NT

Callistocypris rossettii Pinto et al., 2005: NT

Callistocypris thailandensis Savatenalinton \& Martens, 2013: OL

*Callistocypris zlotini Schornikov, 1980: PA, PAC

Candelacypris Baltanás, 2001

*Candelacypris aragonica (Brehm \& Margalef, 1949) Baltanás 2001: PA Eucypris aragonica Brehm \& Margalef, 1949

Candobrasilopsis Higuti \& Martens, 2012

Candobrasilopsis acutis Higuti \& Martens, 2014: NT

Candobrasilopsis anisitsi (Daday, 1905) Higuti \& Martens 2012: NT

Candonopsis anisitsi Daday, 1905

Latinopsis anisitsi (Daday, 1905) Karanovic \& Datry 2009

Candobrasilopsis brasiliensis (Sars, 1901) Higuti \& Martens 2012b: NT Candonopsis brasiliensis Sars, 1901

Candobrasilopsis elongata Higuti \& Martens, 2014: NT

*Candobrasilopsis rochai Higuti \& Martens, 2012: NT

Candocyprinotus Delorme, 1970

? Syn. of Eucypris (Vávra, 1891) Daday, 1900 (fide Smith \& Horne 2016)

*Candocyprinotus ovatus Delorme, 1970: NA

Candona Baird, 1845

Syn.: Mazepovacandona Karanovic \& Sitnikova, 2017 (partim) syn. nov.

Candona acricauda Mikulić, 1961: PA

Candona acuta Hoff, 1942: NA

Candona acutula Delorme, 1967: NA

Candona altoides Petkovski, 1961: PA

Candona amanda Mazepova, 1982: PA

Candona anceps Ekman, 1914: PA

Candona aotearoa Chapman, 1963: AU

Candona araucana Löffler, 1961: NT

Candona arenosa Mazepova, 1982: PA

Candona bimucronata Klie, 1937: PA

Candona birsteini Mazepova, 1990: PA

Candona bradyi Hartwig, 1898: PA

Candona caledoniae Brady, 1910: PA

Candona camelus Schornikov, 1966: PA

* Candona candida (O.F. Müller, 1776) Baird 1845: NA, PA

Cypris candida O.F. Müller, 1776

Syn.: ? Candona studeri Kaufmann, 1900 (fide Meisch 2000)

Candona candida var. humilis Ekman, 1914

Candona capsularis Klie, 1935: NT

Candona crogmaniana Turner, 1894: NA

Syn.: Candona recticauda Sharpe, 1897 (fide Karanovic 2006)

Syn.: Candona reflexa Sharpe, 1897 (fide Karanovic 2006) 
Candona cyproides (Daday, 1905) Lüttig 1962: NT

Eucandona cyproides Daday, 1905

Candona dadayi G.W. Müller, 1912: PA

Nom. nov. pro Candona reticulata Daday, 1897 nec Hejjas, 1894

Candona dalmatina Petkovski et al., 2002: PA

Candona decora Furtos, 1933: NA

Syn.: Candona fossulensis Hoff, 1942 (fide Delorme 1970)

Syn.: Candona facetus Delorme, 1970 (fide Karanovic 2006)

Candona dedelica Petkovski, 1969: PA

Candona delawarensis Turner, 1894: NA

Candona deltoides Mazepova, 1990: PA

Candona demissa Mazepova, 1990: PA

Candona depressa Klie, 1939: PA

Candona directa Bronstein, 1947: PA

Mazepovacandona directa (Bronstein, 1947) Karanovic \& Sitnikova 2017

Candona dryschenkoi Mazepova, 1990: PA

Candona dybowskii Mazepova, 1990: PA

Candona elegans Bronstein, 1928: PA

Candona elpatiewskyi (Daday, 1906) Bronstein, 1928: PA

Eucandona elpatiewskyi Daday, 1906

Candona expansa Mikulić, 1961: PA

Candona falcata Alm, 1914: NA, PA

Candona fasciolata Petkovski, 1961: PA

Candona flava Mazepova, 1984: PA

Candona fluctigera Mazepova, 1990: PA

Candona formosa Mikulić, 1961: PA

Candona fossiliformis Mazepova, 1970: PA

Candona foviolata Dobbin, 1941: NA

Candona galinae nom. nov.: PA

Nom. nov. pro Candona rara Mazepova, 1990 nec G.W. Müller, 1900

Candona godlewskii Mazepova, 1984: PA

Mazepovacandona godlewskii (Mazepova, 1984) Karanovic \& Sitnikova 2017

Candona goricensis Mikulić, 1961: PA

Candona gracilenta Mazepova, 1990: PA

Candona grizea Mazepova, 1982: PA

Candona hadzistei Petkovski et al., 2002: PA

Nom. nov. pro Candona parvula Mikulić, 1961 nec Sars, 1926

Candona hartmanni Petkovski, 1969: PA

Candona holmesi Petkovski, 1960: PA

Candona humilis Bronstein, 1947: PA

Candona ikpikpukensis (Swain, 1963) Delorme 1968: NA

Pseudocandona ikpikpukensis Swain, 1963

Candona iliensis Mandelstam, 1962: PA

Candona illiesi McKenzie, 1971: AU

Typhlocypris (Typhlocypris) illiesi (McKenzie, 1971) Karanovic 2005

Candona improvisa Ostermeyer, 1937: PA

Syn.: Candona devexa Kaufmann, 1900 (nom. oblitum; fide Meisch 2000)

Candona inaequivalvis Sars, 1899: PA

Candona incarum (Moniez, 1889) Martens \& Behen 1994: NT

Cypris incarum Moniez, 1889 
Candona inexpecta Chapman, 1963: AU (see Taxonomic notes)

Candona inopinata Furtos, 1933: NA

Syn.: Candona truncata Furtos, 1933 (fide Karanovic 2006)

Syn.: Candona indigena Hoff, 1942 (fide Karanovic 2006)

Candona insularis Mazepova, 1985: PA

Candona intermedia Furtos, 1933: NA

Candona intersita Mazepova, 1990: PA

Candona ivanowi Mazepova, 1984: PA

Candona jordeae Petkovski et al., 2002: PA

Nom. nov. pro Candona lucida Petkovski, 1959 nec Schneider, 1959

Candona kazminae Konovalova, 2012: PA

Candona keiseri Bronstein, 1929: PA

Candona korjakovi Mazepova, 1982: PA

Candona krochini Bronstein, 1947: PA

Candona kuchurganica Kovalenko, 1988: PA

Candona (Eucandona) kuchurganica Kovalenko, 1988

Candona laciniata Ekman, 1908: PA

Candona lamakini Mazepova, 1990: PA

Candona larvaeformis Bronstein, 1947: PA

Candona larvaeformisoida Mazepova, 1990: PA

Candona larvaeformisoida larvaeformisoida Mazepova, 1990

Candona larvaeformisoida minuta Mazepova, 1990

Candona lepnevae Bronstein, 1947: PA

Candona limosa Mazepova, 1990: PA

Candona limpida Mazepova, 1984: PA

Candona lingulata Cole, 1965: NA

Candona litoralis Mikulić, 1961: PA

Candona longiformis Mazepova, 1990: PA

Candona longula Mazepova, 1985: PA

Candona lychnitis Petkovski, 1969: PA

Candona macedonica Mikulić, 1961: PA

Candona maloemorensis nom. nov.: PA

Nom. nov. pro Candona intermedia Bronstein, 1947 nec Furtos, 1933

Candona margaritana Mikulić, 1961: PA

Candona marginata Klie, 1942: PA

Candona marginatoides Petkovski, 1960: PA

Candona mazepovae nom. nov.: PA

Nom. nov. pro Candona procera Mazepova, 1982 nec Straub, 1952

Candona media Klie, 1939: PA

Candona meerfeldiana Scharf, 1983: PA

Candona memoranda Mazepova, 1990: PA

Candona meridionalis Petkovski, 1958: PA

Syn.: Candona angulata f. meridionalis Petkovski, 1958 (fide Mazzini et al. 2015)

Candona microdorsoconcava Mazepova, 1984: PA

Candona modesta Mazepova, 1984: PA

Candona montenigrina Petkovski, 1961: PA

Candona muelleri Hartwig, 1899: PA

Candona muelleri muelleri Hartwig, 1899

Candona muelleri jakutica Pietrzeniuk, 1977

Candona muriformis Mazepova, 1984: PA 
Candona natronophila Petkovski, 1969: PA

Candona oblonga Sars, 1899: PA

Candona ohioensis Furtos, 1933: NA

Candona ohrida Holmes, 1937: PA

Syn.: Candona cristatella Klie, 1939 (fide Petkovski et al. 2002)

Syn.: Candona sublitoralis Mikulić, 1961 (fide Petkovski et al. 2002)

Candona orbiculata Mazepova, 1990: PA

Mazepovacandona orbiculata (Mazepova, 1990) Karanovic \& Sitnikova 2017

Candona ovalis Mikulić, 1961: PA

Candona paionica Petkovski, 1958: PA

Syn.: Candona paionica minor Petkovski, 1960 (fide Mazzini et al. 2015)

Candona paloskii Petkovski et al., 2002: PA

Candona paraohioensis Staplin, 1963: NA

Candona parva Daday, 1905: NT

Candona peircei Turner, 1895: NA

Syn.: Candona eriensis Furtos, 1933 (fide Karanovic 2006)

Candona peterseni Caraion, 1979: PA

Candona picta Mazepova, 1990: PA

Candona prava Mazepova, 1984: PA

Candona quasiakaina Karanovic \& Lee, 2012: PA

Candona quasiincarum Karanovic \& Datry, 2009: NT

Candona rupestris Bronstein, 1947: PA

Candona rupestris rupestris Bronstein, 1947

Candona rupestris dissona Mazepova, 1990

Candona sanociensis Sywula, 1971: PA

Syn.: Candona dancaui Danielopol, 1973 (fide Meisch 2000)

Candona schweyeri Schornikov, 1964: PA

Nom. nov. pro Candona elongata (Schweyer, 1949) nec Herrick, 1879

Candona semilunaris Bronstein, 1930: PA

Candona semilunaris semilunaris Bronstein, 1930

Candona semilunaris dignitosa Mazepova, 1990

Candona sensibilis Bronstein, 1947: PA

Candona sensibilis sensibilis Bronstein, 1947

Candona sensibilis profunda Mazepova, 1990

Candona sharpei Hoff, 1942: NA

Candona sibirica G.W. Müller, 1912: PA

Nom. nov. pro Candona fragilis Sars, 1899 nec Hartwig, 1898

Candona sigmoides Sharpe, 1897: NA

Syn.: Candona simpsoni Sharpe, 1897 (fide Karanovic 2006)

Syn.: Candona scopulosa Furtos, 1933 (fide Delorme 1970)

Candona sillae Karanovic \& Lee, 2012: PA

Candona sinaidae Mazepova, 1990: PA

Candona spicata Mazepova, 1982: PA

Mazepovacandona spicata (Mazepova, 1982) Karanovic \& Sitnikova 2017

Candona stankovici Mazepova, 1990: PA

Candona strumicae Petkovski, 1959: PA

Candona subacuminata Delorme, 1970: NA

Candona subgibba Sars, 1926: NA

Candona subtriangulata Benson \& McDonald, 1963: NA

Syn.: Candona houghi Staplin, 1963 (fide Delorme 1970) 
Candona suburbana Hoff, 1942: NA

Candona tahoensis Ferguson, 1966: NA

Candona thermalis (Daday, 1900) G.W. Müller 1912: PA

Eucandona rostrata var. thermalis Daday, 1900

Candona trajani Krstić, 1972: PA

Nom. nov. pro Candona alta Klie, 1939 nec Zalanyi, 1929

Candona trapeziformis Klie, 1939: PA

Candona triangulata Klie, 1939: PA

Candona tuberculata (Daday, 1906) Bronstein 1947: PA

Eucandona tuberculata Daday, 1906

Candona uliginosa Furtos, 1933: NA

Syn.: Candona hoffi Ferguson, 1953 (fide Karanovic 2006)

Candona unguiculata Bronstein, 1930: PA

Candona unimoda Mazepova, 1984: PA

Candona uschunica Mazepova, 1990: PA

Candona ustlenski Semenova, 1996: PA

Candona vernalis Fuhrmann, 2008: PA

Candona vidua Klie, 1942: PA

Candona virgata Mazepova, 1985: PA

Candona walukani Mazepova, 1984: PA

Candona wasilievae Mazepova, 1990: PA

Candona wedgewoodi Lowndes, 1932: PA

Candona weltneri Hartwig, 1899: PA, NA

Syn.: Candona weltneri var. obtusa G.W. Müller, 1900 (fide Meisch 2000)

Candona xizangensis Huang, 1982: PA

Candonocypris Sars, 1894

Candonocypris caledonica (Méhes, 1939) De Deckker 1981: AU, PA, PAC

Herpetocypris caledonica Méhes, 1939

Candonocypris deeveyi Tressler, 1954: NA (see Taxonomic notes)

Candonocypris fitzroyi McKenzie, 1966: AU (see Taxonomic notes)

Candonocypris incosta De Deckker, 1981: AU, PAC

Candonocypris novaezelandiae (Baird, 1843) Eagar 1971: AT, AU, PAC, PA

Cypris novaezelandiae Baird, 1843

Syn.: * Candonocypris candonioides (King, 1855) (fide Eagar 1971)

Syn.: Cypris candonioides King, 1855 (fide Eagar 1971)

Syn.: Cypris sydneia King, 1855 (fide De Deckker 1981)

Syn.: Candonocypris assimilis Sars, 1894 (fide De Deckker 1981)

Candonocypris pascheri (Brehm, 1929) Martens \& Savatenalinton 2011: AU (see Taxonomic notes)

Herpetocypris pascheri Brehm, 1929

Candonocypris sarsi Danforth, 1948: NA

Candonopsis Vávra, 1891

Subgenus Abcandonopsis Karanovic, 2004

Candonopsis (Abcandonopsis) aula Karanovic, 2004: AU

Candonopsis (Abcandonopsis) dedeckkeri Karanovic, 2007: AU

Candonopsis (Abcandonopsis) inaffecta Karanovic, 2007: AU

Candonopsis (Abcandonopsis) indoles Karanovic, 2004: AU

Candonopsis (Abcandonopsis) linnaei Karanovic, 2008: AU

Candonopsis (Abcandonopsis) pilbarae Karanovic, 2007: AU

*Candonopsis (Abcandonopsis) williami Karanovic \& Marmonier, 2002: AU 
Candonopsis (Candonopsis) africana Klie, 1944: PA

Subgenus Candonopsis Vávra, 1891

Candonopsis (Candonopsis) anteroacuta Rome, 1962: PA

Candonopsis (Candonopsis) boui Danielopol, 1978: PA

Candonopsis (Candonopsis) bujukuensis Löffler, 1968: AT

Candonopsis (Candonopsis) dani Karanovic \& Marmonier, 2002: AU

Candonopsis (Candonopsis) fessleri Löffler, 1968: AT

Candonopsis (Candonopsis) fessleri fessleri Löffler, 1968

Candonopsis (Candonopsis) fessleri f. daburui Löffler, 1968

Candonopsis (Candonopsis) hummelincki Broodbakker, 1983: NT

Candonopsis (Candonopsis) kimberleyi Karanovic \& Marmonier, 2002: AU

*Candonopsis (Candonopsis) kingsleii (Brady \& Robertson, 1870) Vávra 1891: NA, NT, PA

Candona kingsleii Brady \& Robertson, 1870

Candonopsis (Candonopsis) mareza Karanovic \& Petkovski, 1999: PA

Candonopsis (Candonopsis) murchisoni Karanovic \& Marmonier, 2002: AU

Candonopsis (Candonopsis) nama Daday, 1913: AT

Candonopsis (Candonopsis) navicula Daday, 1910: AT

Syn.: Candonopsis (Candonopsis) depressa Rome, 1962 (fide Karanovic 2004)

Syn.: Candonopsis (Candonopsis) dorsorecta Rome, 1962 (fide Karanovic 2004)

Candonopsis (Candonopsis) putealis Klie, 1932: OL

Candonopsis (Candonopsis) scourfieldi Brady, 1910: PA

Syn.: Candonopsis parva Sywula, 1968 (fide Meisch 2000)

Syn.: Candonopsis stammeri Nüchterlein, 1969 (fide Meisch 2000)

Candonopsis (Candonopsis) solitaria Vávra, 1895: AT

Candonopsis (Candonopsis) sumatrana Klie, 1932: OL, PA, PAC

Syn.: Candonopsis (Candonopsis) calva Harding, 1962 (fide Karanovic 2004)

Candonopsis (Candonopsis) tenuis (Brady, 1886) Sars 1896: AU, PA, PAC

Candona tenuis Brady, 1886

Candonopsis (Candonopsis) thienemanni Schäfer, 1945: PA

Candonopsis (Candonopsis) transgrediens Brehm, 1923: PA

Candonopsis (Candonopsis) trichota Schäfer, 1945: PA

Candonopsis (Candonopsis) urmilae Gupta, 1988: OL

Candonopsis (Candonopsis) westaustraliensis Karanovic \& Marmonier, 2002: AU

Caribecandona Broodbakker, 1983

Caribecandona ansa Broodbakker, 1983: NT

Caribecandona auricularia Broodbakker, 1983: NT

*Caribecandona trapezoidea Broodbakker, 1983: NT

Cavernocypris cavernosa Smith, 2011: PA

Cavernocypris Hartmann, 1964

Cavernocypris coreana (McKenzie, 1972) Marmonier et al. 1989: PA

Cavernocypris coreana coreana (McKenzie, 1972) Marmonier et al. 1989

'Cypridopsis' coreana coreana McKenzie, 1972

Cavernocypris coreana elongata (McKenzie, 1972) Marmonier et al. 1989

'Cypridopsis' coreana elongata McKenzie, 1972

Cavernocypris danielopoli Smith \& Kamiya, 2017: PA

Cavernocypris subterranea (Wolf, 1920) Wouters 1983: NA, PA

Cypridopsis subterranea Wolf, 1920

Syn.: Cypridopsis shadini Akatova, 1950 (fide Marmonier et al. 1989)

Syn.: Cypridopsis subterranea germanica Petkovski, 1962 (fide Löffler 1963b) 
Syn.: *Cavernocypris lindbergi Hartmann, 1964 (fide Marmonier et al. 1989)

Cavernocypris wardi Marmonier et al., 1989: NA

\section{Centrocypris Vávra, 1895}

Centrocypris bhagirathiae Battish, 1981: OL

*Centrocypris horrida (Vávra, 1895) Daday 1900: AT, OL

Cypris (Centrocypris) horrida Vávra, 1895

Centrocypris indica Gupta, 1991: OL

Centrocypris jakubskii Grochmalicki, 1913: AT

Centrocypris madani Battish, 1981: OL

Centrocypris margaritifera G.W. Müller, 1898: AT

Centrocypris matthaii (Arora, 1931) Victor \& Fernando 1979b: OL

Eurycypris matthaii Arora, 1931

Centrocypris viridis Neale, 1976: OL

Chelocythere Hart \& Hart, 1967

*Chelocythere kalganensis Hart \& Hart, 1967: AU

Chlamydotheca Saussure, 1858

Cypris (Chlamydotheca) Saussure, 1858

Syn.: Pachycypris Claus, 1892 (fide G.W. Müller 1912)

Chlamydotheca angulata Klie, 1939: NT

Chlamydotheca arcuata (Sars, 1901) G.W. Müller 1912: NA, NT, OL, PA

Cypris arcuata Sars, 1901

Syn.: Eucypris (Chlamydotheca) bennelong King, 1855 (sensu Daday 1905, wrong identification)

(fide Martens \& Behen 1994)

*Chlamydotheca azteca Saussure, 1858: NA, NT

Chlamydotheca barbadensis Sharpe, 1910: NT

Syn.: Chlamydotheca hummelincki Triebel, 1961 (fide Martens \& Savatenalinton 2011)

Syn.: Chlamydotheca hummelincki dispar Triebel, 1961 (fide Martens \& Savatenalinton 2011)

Syn.: Chlamydotheca hummelincki nordestina Kotzian, 1961 (fide Martens \& Savatenalinton 2011)

Syn.: Chlamydotheca hummelincki nicoi Martens, 1994 (fide Martens \& Savatenalinton 2011)

Nom. nov. pro C. barbadensis dispar Broodbakker, 1984 nec C. hummelincki dispar

Triebel, 1961

Chlamydotheca bicolor Roessler, 1986: NT

Chlamydotheca calcarata Klie, 1930: NT

Syn.: Chlamydotheca kleerekoperi Tressler, 1950 (fide Martens \& Behen 1994)

Chlamydotheca colombiensis Roessler, 1985: NT

Chlamydotheca dadayi Kotzian, 1974: NT

Chlamydotheca deformis Farkas, 1958: NT

Chlamydotheca mexicana deformis Farkas, 1958

Chlamydotheca deformis deformis Farkas, 1958

Chlamydotheca deformis cearaensis Kotzian, 1974

Chlamydotheca deformis colombiensis Roessler, 1986

Chlamydotheca elegans Roessler, 1986: NT

Chlamydotheca flexilis (Brady, 1902) G.W. Müller 1912: NA, NT

Cypris flexilis Brady, 1902

Chlamydotheca iheringi (Sars, 1901) Klie, 1930: NT

Cypris iheringi Sars, 1901

Syn.: Chlamydotheca mckenziei Kotzian, 1974 (fide Martens et al. 1998) 
MEISCH C. et al., Global checklist of extant non-marine Ostracoda

Chlamydotheca incisa (Claus, 1892) Sharpe, 1910: NT, PA

Pachycypris incisa Claus, 1892

Syn.: Cypris (Eucypris) limbata Wierzejski, 1893 (fide Martens \& Behen 1994)

Syn.: Cypris labiata Sars, 1901 (fide Martens \& Behen 1994)

Syn.: Chlamydotheca alegrensis Tressler, 1950 (fide Martens \& Behen 1994)

Syn.: Chlamydotheca incisa clara Roessler, 1986 (fide Martens \& Savatenalinton 2011)

Syn.: Chlamydotheca incisa obscura Roessler, 1986 (fide Martens \& Savatenalinton 2011)

Chlamydotheca leuckarti (Claus, 1892) Sharpe 1910: NT

Pachycypris leuckarti Claus, 1892

Chlamydotheca leuckarti var. pallida (Claus, 1892) G.W. Müller 1912

Pachycypris leuckarti var. pallida Claus, 1892

Chlamydotheca llanoensis Pierce, 1988: NA

Chlamydotheca magdalenensis Roessler, 1986: NT

Chlamydotheca manguerensis Kotzian, 1974: NT

Chlamydotheca mayor Roessler, 1986: NT

Chlamydotheca mexicana Sharpe, 1903: NA

Chlamydotheca pseudobrasiliensis Martens, 1994: NT

Nom. nov. pro Chlamydotheca braziliensis Tressler, 1950 nec C. brasiliensis (Lubbock, 1855)

Chlamydotheca riograndensis Kotzian, 1974: NT

Chlamydotheca riograndensis riograndensis Kotzian, 1974

Chlamydotheca riograndensis trichosa Roessler, 1986

Chlamydotheca rudolphi Triebel, 1939: NT

Chlamydotheca speciosa (Dana, 1849) Sharpe 1910: NA, NT

Cypris speciosa Dana, 1849

Syn.: Cypris herricki Turner, 1892 (fide G.W. Müller 1912)

Chlamydotheca spectabilis (Sars, 1901) Triebel 1939: NT

Cypris spectabilis Sars, 1901

Chlamydotheca symmetrica (Vávra, 1898) Sharpe 1910: NT

Cypris symmetrica Vávra, 1898

Chlamydotheca texasiensis (Baird, 1862) G.W. Müller 1912: NA, NT

Cypris texasiensis Baird, 1862

Chlamydotheca tolimensis Roessler, 1986: NT

Chlamydotheca unispinosa (Baird, 1862) Furtos 1936a: NA, NT, PAC

Cypris unispinosa Baird, 1862

Syn.: Candonocypris serrato-marginata (Furtos, 1936) (juvenile, fide Martens \& Behen 1994)

Syn.: Eucypris serrato-marginata Furtos, 1936a (fide Furtos 1936b)

Chlamydotheca vanhoeffeni (Daday, 1911) Kliec 1939: NT

Cypris vanhoeffeni Daday, 1911

Chlamydotheca wrighti Tressler, 1950: NT

Chrissia Hartmann, 1957

Syn.: Gesa Hartmann, 1957 (fide Martens 2001a)

Syn.: Parastenocypris Hartmann, 1964 (fide Martens 2001a)

Chrissia achandii (George \& Martens, 1993) Martens 2001a: OL

Parastenocypris achandii George \& Martens, 1993

Chrissia aldabrae (G.W. Müller, 1898) Martens 2001a: AT

Stenocypris aldabrae G.W. Müller, 1898

Parastenocypris aldabrae (G.W. Müller, 1898) McKenzie 1971

Chrissia ametra (G.W. Müller, 1908) Martens 2001a: AT

Stenocypris ametra G.W. Müller, 1908 
Parastenocypris ametra (G.W. Müller, 1908) McKenzie 1971

Syn.: Stenocypris olivacea Sars, 1924 (fide McKenzie 1971)

Syn.: Stenocypris ametra var. minor G.W. Müller, 1908 (fide Martens 2001b)

Chrissia bhatiai (Mannikeri \& Vaidya, 1990) Martens 2001a: OL

Parastenocypris bhatiai Mannikeri \& Vaidya, 1990

Chrissia bispinosa (G.W. Müller, 1914) Martens 2001a: AT

Stenocypris bispinosa G.W. Müller, 1914

Parastenocypris bispinosa (G.M. Müller, 1914) McKenzie 1971

Syn.: Stenocypris pardalis Sars, 1924 (fide Martens 2001b)

Chrissia biswasi (Deb, 1972) Martens, 2001: OL

Stenocypris biswasi Deb, 1972

Parastenocypris biswasi (Deb, 1972) Harshey \& Victor, 1983

Chrissia canaliculata (Hartmann, 1964) Martens 2001a: OL

Parastenocypris canaliculata Hartmann, 1964

Chrissia ceylonica (Daday, 1898) Neale 1984: OL

Stenocypris ceylonica Daday, 1898

Chrissia cultrata (G.W. Müller, 1900) Martens 2001a: AT

Stenocypris cultrata G.W. Müller, 1900

Parastenocypris cultrata (G.W. Müller, 1900) Harshey \& Victor 1983

Chrissia declivis (Sars, 1924) Martens 2001a: AT

Stenocypris declivis Sars, 1924

Parastenocypris declivis (Sars, 1924) McKenzie 1971

Chrissia dongqianhuensis Kong et al., 2014: PA

Chrissia dyalpurensis Battish, 1998: OL

Chrissia fasciculata (Daday, 1910) Martens \& Savatenalinton 2011: AT, OL Stenocypris fasciculata Daday, 1910

Chrissia fascigera (Sars, 1924) McKenzie 1977: AT

Stenocypris fascigera Sars, 1924

Chrissia fernandoi (Neale, 1976) Martens 2001a: OL

Stenocypris fernandoi Neale, 1976

Parastenocypris fernandoi (Neale, 1976) George \& Martens 1993

Chrissia formosa (Klie, 1938) Okubo 1974: OL, PA

Stenocypris formosa Klie, 1938

Chrissia fuelleborni (Daday, 1910) Martens \& Savatenalinton 2011: AT, OL Stenocypris fuelleborni Daday, 1910

Chrissia goddeerisi (George \& Martens, 1993) Martens 2001a: OL

Parastenocypris goddeerisi George \& Martens, 1993

Chrissia halyi (Ferguson, 1969) Okubo 1974: OL

Stenocypris halyi Ferguson, 1969

Chrissia hodgsoni (Sars, 1924) Martens 2001a: AT, PA

Stenocypris hodgsoni Sars, 1924

Parastenocypris hodgsoni (Sars, 1924) McKenzie 1971

Chrissia humilis (Klie, 1932) Hartmann 1964: NT, OL

Chrissia humilis humilis (Klie, 1932) Hartmann 1964

Stenocypris humilis humilis Klie, 1932

Chrissia humilis indica Hartmann, 1964

Chrissia icosacantha (Lowndes, 1931) Martens 2001a: AT

Stenocypris icosacantha Lowndes, 1931

Parastenocypris icosacantha (Lowndes, 1931) Harshey \& Victor 1983

Chrissia junodi (Delachaux, 1919) Martens 1984: AT

Stenocypris junodi Delachaux, 1919 
Chrissia junodi junodi (Delachaux, 1919) Martens 1984

Syn.: Stenocypris urodonta Rome, 1965 (fide Martens 1984)

Chrissia junodi monardi (Brehm, 1938) Klie, 1944

Syn.: Stenocypris monardi Brehm, 1938 (fide Martens 1984)

Chrissia khopoliensis (Deb, 1983) Martens \& Savatenalinton 2011: OL

Stenocypris khopoliensis Deb, 1983

Chrissia krishnakantai (Deb, 1972) Victor \& Fernando 1979b: OL

Stenocypris krishnakantai Deb, 1972

*Chrissia levetzovi Hartmann, 1957: AT

Syn.: Gesa dubia Hartmann, 1957 (fide Martens 1984)

Chrissia monodi (Gauthier, 1930) Martens 1984: AT

Stenocypris monodi Gauthier, 1930

Chrissia nealei (Mannikeri \& Vaidya, 1990) Martens 2001a: OL

Parastenocypris nealei Mannikeri \& Vaidya, 1990

Chrissia ousmana Ghetti, 1972: PA

Chrissia pectinata (Sars, 1924) Martens 2001a: AT

Stenocypris pectinata Sars, 1924

Parastenocypris pectinata (Sars, 1924) McKenzie 1971

Chrissia perarmata (Brady, 1904) Martens 2001a: AT

Stenocypris perarmata Brady, 1904

Parastenocypris perarmata (Brady, 1904) McKenzie 1971

Chrissia sinuata (G.W. Müller, 1898) Martens 2001a: AT

Stenocypris sinuata G.W. Müller, 1898

Parastenocypris sinuata (G.W. Müller, 1898) Martens 1984

Chrissia smaragdina (Sars, 1924) Martens 2001a: AT

Stenocypris smaragdina Sars, 1924

Parastenocypris smaragdina (Sars, 1924) McKenzie 1971

Syn.: Stenocypris anisoacantha Rome, 1965 (fide Martens 2001b)

Chrissia spinosa (Tressler, 1937) Martens \& Savatenalinton 2011: OL

Stenocypris spinosa Tressler, 1937

Chrissia vittata Okubo, 1974: OL, PA

Chrissia werneri (Daday, 1910) Martens 2001a: PA

Stenocypris werneri Daday, 1910

Parastenocypris werneri (Daday, 1910) Martens 1993

Comalcandona Külköylüoğlu \& Gibson 2018

*Comalcandona tressleri Külköylüoğlu \& Gibson, 2018: NA

Cryptocandona Kaufmann, 1900

Cryptocandona angustissima (Ekman, 1914) Alm 1915: PA

Candona angustissima Ekman, 1914

Cryptocandona brehmi (Klie, 1934) Bronstein 1947: PA

Candona brehmi Klie, 1934

Cryptocandona dudichi (Klie, 1930) Brehm 1953: PA

Candona dudichi Klie, 1930

Cryptocandona kieferi (Klie, 1938) Danielopol 1980: PA

Candona kieferi Klie, 1938

Cryptocandona kieferi kieferi (Klie, 1938) Danielopol 1980

Syn.: Cryptocandona juvavi Brehm, 1953 (fide Meisch 2000)

Cryptocandona kieferi danubialis Namiotko et al., 2005 
Cryptocandona kieferi dobrogensis Namiotko et al., 2005

Cryptocandona leruthi (Klie, 1936) Danielopol 1980: PA

Candona leruthi Klie, 1936

Cryptocandona longipes (Ekman, 1908) G.W. Müller 1912: PA

Candona longipes Ekman, 1908

Cryptocandona matris (Sywula, 1976) Danielopol 1980: PA

Candona matris Sywula, 1976

Cryptocandona phreaticola (Klie, 1927) Brehm 1953: PA

Candona phreaticola Klie, 1927

Cryptocandona pygmaea (Ekman, 1908) G.W. Müller 1912: PA

Candona pygmaea Ekman, 1908

Cryptocandona reducta (Alm, 1914) Sars 1925: PA

Candona reducta Alm, 1914

Cryptocandona smithi Karanovic \& Lee, 2012: PA

Cryptocandona tsukagoshii Smith, 2011: PA

*Cryptocandona vavrai Kaufmann, 1900: PA

Cubacandona Broodbakker, 1983

*Cubacandona cubensis (Danielopol, 1978) Broodbakker 1983: NT

Candonopsis cubensis Danielopol, 1978

Cyclocypris Brady \& Norman, 1889

Syn.: Cyclocypria Dobbin, 1941 (fide Dobbin Evenson 1969)

Syn.: Cyclocypris (Leucocypris) Jančařík, 1949 (fide Meisch 2000)

Syn.: Cyclocypris (Laevicypris) Krstić, 1995 (fide Meisch 2000)

Cyclocypris ampla Furtos, 1933: NA

Cyclocypris anacola Smith et al., 2015: PA

Cyclocypris brevisetosa (Bronstein, 1925) Meisch 1987: PA

Cyclocypris serena brevisetosa Bronstein, 1925

Cyclocypris diebeli Absolon, 1973: PA

Cyclocypris forbesi Sharpe, 1897: NA, PA

*Cyclocypris globosa (Sars, 1863) Brady \& Norman 1889: NA, PA

Cypris globosa Sars, 1863

Syn.: Cypris cinerea Brady, 1868 (fide G.W. Müller 1912)

Syn.: Cyclocypris dispersa G.W. Müller, 1912

Nom. nov. pro Cypris globosa G.O. Sars, 1863 nec Dieffenbach, 1843

Cyclocypris helocrenica Fuhrmann \& Pietrzeniuk, 1990: PA

Cyclocypris kincaidia (Dobbin, 1941) Dobbin Evenson 1969: NA

Cyclocypria kincaidia Dobbin, 1941 (see Taxonomic notes)

Cyclocypris klughi nom. nov.: NA

Nom. nov. pro Cyclocypris castanea Klugh, 1923 nec Brady, 1913

Cyclocypris laevis (O.F. Müller, 1776) Sars 1890: NA, PA

Cypris laevis O.F. Müller, 1776

? Syn.: Cyclocypris laevis labialis Sywula, 1981 (fide Meisch 2000)

? Syn.: Cyclocypris labialis Sywula, 1981 (sensu Fuhrmann \& Goth 2011, fide Meisch 2000)

? Syn.: Cyclocypris humilis Pietrzeniuk, 1985 (sensu Fuhrmann \& Goth 2011, fide Meisch 2000)

Cyclocypris mediosetosa Meisch, 1987: PA

Cyclocypris nahcotta Dobbin, 1941: NA

Cyclocypris ovoides (Alm, 1914) Fuhrmann, 2008: PA

Cyclocypris globosa var. ovoides Alm, 1914 
MEISCH C. et al., Global checklist of extant non-marine Ostracoda

Cyclocypris ovum (Jurine, 1820) Sars 1903a: NA, PA

Monoculus ovum Jurine, 1820

? Syn.: Cyclocypris pygmaea Croneberg, 1894 (fide Meisch 2000)

? Syn.: Cyclocypris ovum forma pygmaea Croneberg, 1894 (fide Meisch 2000)

? Syn.: Cyclocypris impressopunctata Hirschmann, 1909 (fide Hollwedel \& Scharf 1996)

? Syn.: Cyclocypris ovum forma impressopunctata Hirschmann, 1909 (fide Meisch 2000)

? Syn.: Cyclocypris taubachensis Diebel \& Pietrzeniuk, 1984 (fide Hollwedel \& Scharf 1996)

Cyclocypris pusilla Sars, 1895: AT

Cyclocypris scrobiculata Klie, 1936: PA

Cyclocypris serena (Koch, 1838) Sars 1890: NA, PA

Cypris serena Koch, 1838

Syn.: Cyclocypris serena guttata Brehm, 1923 (fide Martens \& Savatenalinton 2011)

Syn.: ? Cyclocypris drastichi Jančařík, 1949 (fide Meisch 2000)

Cyclocypris sharpei Furtos, 1933: NA

Syn.: Cyclocypris cruciata Furtos, 1935 (fide Karanovic 2011)

Cyclocypris vinyardi Külköylüoğlu, 2008: NA

Cyclocypris washingtoniensis Dobbin, 1941: NA

Cyclocypris wyomingensis Ferguson, 1966: NA

Cymocythere clavata Crawford, 1965: NA

Cymocythere Hart, 1962

*Cymocythere cyma (Hobbs \& Walton, 1960) Hart 1962: NA

Entocythere cyma Hobbs \& Walton, 1960

Cymocythere gonia Hobbs \& Hart, 1966: NA

Cypretta Vávra, 1895

Cypretta alagarkoilensis Victor \& Michael, 1975: OL

Cypretta baylyi McKenzie, 1966: AU

Cypretta bilicis Furtos, 1936: NA

Cypretta bradyi Martens \& Savatenalinton, 2011: AT, NT

Nom. nov. pro Cypretta sarsi Brady, 1902 nec Cypretta sarsi (Moniez, 1892)

Cypretta brevisaepta Furtos, 1934: NA, NT

Cypretta brevisaepta var. sarta Furtos, 1936

Cypretta brevispina Farkas, 1959: NT

Nom. nov. pro Cypretta reticulata Danforth, 1948 nec Lowndes, 1932

Cypretta campechensis Cohuo-Durán et al., 2013: NT

Cypretta comitisroseni (Brehm, 1924) Furtos 1936: NT

Eucypris comitisroseni Brehm, 1924

Cypretta cordata Klie, 1938: AT

Cypretta costata G.W. Müller, 1898: AT, NT, PA

Cypretta elongata Macario-González et al., 2018: NT

Cypretta fontinalis Hartmann, 1964: OL

Cypretta foveata Hartmann, 1964: OL

Cypretta gargi Deb, 1983: OL

Cypretta globosa (Brady, 1886) Neale 1977: OL

Cypridopsis globosa Brady, 1886

Cypretta globula (Sars, 1889) G.W. Müller 1898: AT, AU, OL, PA, PAC

Cypridopsis globula Sars, 1889

Cypretta globulosa (Sharpe, 1910) Sohn \& Kornicker 1973: NA

Cypris globulosa Sharpe, 1910 
Cypretta godeti (Delachaux, 1928) Furtos 1936: NT

Eucypris godeti Delachaux, 1928

Cypretta hirsuta Henry, 1923: AU

Cypretta infesta Klie, 1941: NT

Cypretta intonsa Furtos, 1936: NA

Cypretta judayi Tressler, 1937: AU

Cypretta karanovicae Smith et al., 2015: PA

Cypretta kawatai Sohn \& Kornicker, 1972: NT

Cypretta kenyensis Klie, 1939: AT

Cypretta lindbergi Hartmann, 1964: PA

Cypretta longidactyla Victor \& Fernando, 1981: OL

Cypretta lutea McKenzie, 1966: AU

Cypretta margalefi Brehm, 1949: NT

Cypretta maya Cohuo-Durán et al., 2013: NT

Cypretta minna (King, 1855) Daday 1900: AT, AU, PA

Cypris minna King, 1855

Cypretta murati Gauthier, 1939: AT, PA

Cypretta nigra Furtos, 1936: NA

Cypretta nukuhivana Furtos, 1934: NA, PAC

Cypretta obfuscata Victor \& Fernando, 1981: OL

Cypretta oxyuris Daday, 1910: AT

Cypretta papuana Vávra, 1901: AU, PAC

Cypretta patialaensis Battish, 1982: OL

Cypretta raciborskii (Grochmalicki, 1915) Furtos 1936: OL

Cypridopsis raciborskii Grochmalicki, 1915

Cypretta ramai Deb, 1984: OL

Cypretta remota (Vávra, 1906) G.W. Müller 1912: OL

Cypridella remota Vávra, 1906

Cypretta rotunda Ghetti, 1972: PA

Cypretta sarsi (Moniez, 1892) Victor \& Fernando 1981: OL

Cypris sarsi Moniez, 1892

Cypretta schubarti Farkas, 1959: NT

Cypretta seurati Gauthier, 1929: AT, NT, OL, PA, PAC

Syn.: Cyprella dubiosa Daday, 1900 (unused older synonym) (fide Meisch et al. 2007)

Syn.: Cypretta dubiosa (Daday, 1900) G.W. Müller 1912

Cypretta spinosa Cohuo-Durán et al., 2013: NT

*Cypretta tenuicauda (Vávra, 1895) G.W. Müller 1898: AT, AU

Cypridopsis (Cypretta) tenuicauda Vávra, 1895

Cypretta turgida (Sars, 1896) Daday 1900: AT, AU, NA, OL, PA, PAC

Cypridopsis turgida Sars, 1896

Cypretta viridis (Thomson, 1879) Daday 1900: AU

Cypris viridis Thomson, 1879

Cypretta vivacis Würdig \& Pinto, 1993: NT

Cypretta yapinga De Deckker, 1982: AU

Cypria Zenker, 1854

Syn.: Candocypria Furtos, 1933 (fide Karanovic 2011)

Syn.: Bentocypria Kovalenko, 1987 (fide Karanovic 2011)

Syn.: Keysercypria Karanovic, 2011 (partim) syn. nov.

Cypria asiatica Karanovic \& Lee, 2012: PA

Cypria benoiti Kiss, 1959: AT 
Cypria bicolor Petkovski \& Meisch, 1994: PA

Cypria brevisetigera Cole, 1965: NA, OL

Cypria cavernae Wagenleitner, 1990: PA

Cypria curvifurcata Klie, 1923: NA, PA

* Cypria exsculpta (Fischer, 1855) Brady \& Norman 1889: PA

Cypris exsculpta Fischer, 1855

Syn.: Cypris elegantula Lilljeborg, 1853 (fide G.W. Müller 1912; unused older syn.)

Syn.: Cypris striolata Brady, 1864 (fide G.W. Müller 1912)

? Syn.: Cypria granulata (Robertson, 1880) Brady \& Norman 1889 (fide G.W. Müller 1912)

Cypria javana G.W. Müller, 1906: OL

Cypria karamani Petkovski, 1976: PA

Cypria kliei Martens \& Savatenalinton, 2011: OL (see Taxonomic notes)

Syn.: Cypria javana pelagica Klie, 1932

Nom. nov. pro Cypria pelagica Klie, 1932 nec Brehm, 1932

Cypria kolymensis Akatova, 1975: PA

Cypria konishii Smith \& Kamiya, 2006: PA

Cypria lacrima Külköylüoğlu et al., 2017: NA

Cypria lacustris Lilljeborg, 1890 (in Sars 1890): NA, PA (see Taxonomic notes)

Cypria lata (Dubowsky, 1929) Kovalenko 1987: PA

Cypria curvifurcata var. lata Dubowsky, 1929

Cypria lenticularis G.W. Müller, 1898: AT

Cypria lubeziensis Kovalenko, 1982: PA

Cypria maculata Hoff, 1942: NA

Cypria matzkeae Smith \& Janz, 2008: PA

Cypria mediana Hoff, 1942: NA

Cypria obesa Sharpe, 1897: NA

Cypria obliqua Klie, 1939: PA

Cypria obtusa Klie, 1940: NA, NT, PA

Keysercypria obtusa (Klie, 1940) Karanovic 2011

Cypria ophtalmica (Jurine, 1820) Brady \& Norman 1889: NA, PA

Monoculus ophtalmicus Jurine, 1820

Syn.: Cypris compressa Baird, 1835 (fide G.W. Müller 1912)

Syn.: Cypris tenera Koch, 1837 (fide G.W. Müller 1912)

Syn.: Cypris punctata Koch, 1838 (fide G.W. Müller 1912)

Syn.: Cypris elegantula Fischer, 1851 (fide G.W. Müller 1912)

Syn.: Cypria ophtalmica var. punctata Monard, 1920 (fide Meisch 2000)

Cypria osburni (Furtos, 1933) Karanovic 2011: NA

Candocypria osburni Furtos, 1933

Syn.: Cypria fontana Cole, 1965 (fide Karanovic 2011)

Cypria palustera Furtos, 1935: NA

Cypria pellucida Sars, 1901: NA, NT

Keysercypria pellucida (Sars, 1901) Karanovic,2011

Cypria polessica Kovalenko, 1982: PA

Cypria pseudocrenulata Furtos, 1936: NA, NT

Cypria (Cypria) pseudocrenulata Furtos, 1936

Cypria pusilla Sars, 1896: AU

Cypria reptans Bronstein, 1928: PA

Syn.: Cypria stygia Klie, 1935 (fide Petkovski 1976)

Syn.: Cypria helokrenica Kantorek \& Absolon, 1975 (fide Petkovski 1976)

Cypria sharmai Battish, 1985: OL

Cypria sketi Petkovski, 1976: PA 
Cypria spinifera Tressler, 1937: OL

Cypria subsalsa (Redeke, 1936) Petkovski, 1976: PA

Cypria ophthalmica var. subsalsa Redeke, 1936

Cypria sywulai Meisch, 2000: PA (see Taxonomic notes)

Nom. nov. pro Cypria lata Sywula, 1981 nec Dubowsky, 1929

Syn.: Cypria ophtalmica var. lata Sywula, 1981 (fide Meisch 2000)

Syn.: Cypria lata Meisch \& Forró, 1998 (fide Meisch 2000)

Syn.: Cypria sywulae Meisch, 2000 (incorrect spelling; see Taxonomic notes)

Cypria turneri Hoff, 1942: NA

Cypricercus Sars, 1895

Cypricercus acanthigera (G.W. Müller, 1912) Martens \& Behen 1994: AT, NT

Strandesia acanthigera G.W. Müller, 1912

Nom. nov. pro Strandesia mucronata (Sars, 1901) nec Claus, 1892

Syn.: Neocypris mucronata Sars, 1901 (fide Martens \& Behen 1994)

Syn.: Cypricercus episphaena G.W. Müller, 1908 (fide Martens \& Behen 1994)

Cypricercus ariariensis (Roessler, 1986) Martens \& Behen 1994: NT

Strandesia ariariensis Roessler, 1986

Cypricercus centrurus (Klie, 1940) Martens \& Behen 1994: NT

Strandesia centrura Klie, 1940

Cypricercus cheboyganensis Ferguson, 1957: NA

*Cypricercus cuneatus Sars, 1895: AT

Cypricercus elegans (Roessler, 1986) Martens \& Behen 1994: NT

Strandesia elegans Roessler, 1986

Cypricercus indrani Deb, 1983: OL

Cypricercus inermis (Brady, 1904) Martens et al. 1998: AT

Cypris inermis Brady, 1904

Cypricercus maculatus G.W. Müller, 1908: AT

Cypricercus munshii Deb \& Nasar, 1977: OL

Cypricercus sanguineus Chapman, 1963: AU

Cypricercus setosus Farkas, 1957: OL

Cypricercus unicornis De Deckker, 1981: AU

Cypricercus variabilis (Roessler, 1986) Martens \& Behen 1994: NT

Strandesia variabilis Roessler, 1986

Cypricercus vietsi Farkas, 1957: OL

Cypricercus xhosa Savatenalinton \& Martens, 2009: AT

Cypriconcha alba Dobbin, 1941: NA

Cypriconcha Sars, 1926

* Cypriconcha barbata (Forbes, 1893) Sars 1926: NA

Cypris barbata Forbes, 1893

Cypriconcha gnathostoma Ferguson, 1967: NA

Cypriconcha hypsophila Díaz \& Lopretto, 2009: NT

Cypriconcha ingens Delorme, 1967: NA

Cypriconcha macra Blake, 1931: NA

Nom. nov. pro Cypris grandis Chambers, 1877 nec Reuss, 1844

Syn.: Cypriconcha grandis (Chambers, 1877) (fide Blake 1931)

Syn.: Cypriconcha gigantea Dobbin, 1941 (fide Dobbin Evenson 1969)

Cypriconcha pseudoingens (Delorme, 1969) McKenzie 1982a: NA

Megalocypris pseudoingens Delorme, 1969

Cypriconcha steveni McKenzie, 1982: NA 
Cyprideis aciculata Wouters \& Martens, 2007: AT

Cyprideis Jones, 1857

Cyprideis americana (Sharpe, 1908) Sandberg \& Plusquellec 1974: NA

Cythere americana Sharpe, 1908

Cyprideis anatolica Bassiouni, 1979: PA

Cyprideis (Cyprideis) anatolica Bassiouni, 1979

Cyprideis australiensis Hartmann, 1978: AU, PAC

Cyprideis bairdii Sars, 1866: PA

Cyprideis beaconensis (Le Roy, 1943) Sandberg 1964: NA, NT

Cytheridea beaconensis Le Roy, 1943

Syn.: Cyprideis lengae Hartmann, 1961 (fide Sandberg 1964)

Cyprideis castus Benson, 1959: NT

Cyprideis (Goerlichia) castus Benson, 1959

Cyprideis consobrina (Brady, 1890) McKenzie 1986: PAC

Cythere consobrina Brady, 1890

Cyprideis edentata Klie, 1939: NT

Cyprideis gelica Sandberg \& Plusquellec, 1974: NA

Cyprideis limbocostata Hartmann, 1974: AT

Cyprideis loricata Wouters \& Martens, 2001: AT

Cyprideis mastai Wouters \& Martens, 1994: AT

Cyprideis mexicana Sandberg, 1964: NA

Cyprideis multidentata Hartmann, 1955: NT

Syn.: Cyprideis riograndensis Pinto \& Ornellas, 1965 (fide Sandberg 1964)

Cyprideis pacifica Hartmann, 1957: NT

Cyprideis pedaschenkoi (Daday, 1909) Bronstein 1929: PA

Cytheridea pedaschenkoi Daday, 1909

Cyprideis profunda Wouters \& Martens, 1999: AT

Cyprideis remanei Klie, 1940: AT

Cyprideis romei Wouters \& Martens, 2008: AT

Cyprideis rumongensis Wouters \& Martens, 1994: AT

Cyprideis saetosa Hartmann, 1955: NT

Cyprideis salebrosa Van Den Bold, 1963: NA, NT

Cyprideis salebrosa salebrosa Van Den Bold, 1963

Cyprideis salebrosa hartmanni (Ramirez, 1967) Ornellas \& Würdig 1983

Syn.: Cyprideis hartmanni Ramirez, 1967 (fide Martens \& Behen 1994)

Cyprideis similis (Brady, 1869) van den Bold 1963: NT

Cythere similis Brady, 1869

Syn.: Cyprideis inermis Klie, 1939 (fide Sandberg 1964)

Cyprideis spatula Wouters \& Martens, 1999: AT

Cyprideis stenopora Triebel, 1956: NT

Cyprideis stephensoni Sandberg, 1964: NA

*Cyprideis torosa (Jones, 1850) Jones 1857: AT, AU, NA, PA

Candona torosa Jones, 1850

Syn.: Cytheridea littoralis Brady, 1870 (fide Athersuch et al., 1989)

Syn.: Cytheridea aegyptiaca Daday, 1910 (fide Klie 1935)

Syn.: Cyprideis sohni Bassiouni, 1979 (fide Wouters 2002)

Cyprideis westraliensis McKenzie, 1978: AU 
Cypridopsis Brady, 1867

Syn.: Pionocypris Brady \& Norman, 1896 (fide G.W. Müller 1912)

Cypridopsis acanthodes Rome, 1962: AT

Cypridopsis adusta Sars, 1903: OL, PA

Cypridopsis aequalis Rome, 1962: AT

Cypridopsis arcuata Rome, 1962: AT

Cypridopsis arhiga Cole, 1965: NA

Cypridopsis australis Henry, 1923: AU

Cypridopsis bidentata Sars, 1910: AT

Cypridopsis bisexualis Cole, 1966: NA

Nom. nov. pro Cypridopsis compressa Cole, 1965 nec Chapman, 1934 (fossil species)

Cypridopsis brevisetosa Klie, 1943: AT

Cypridopsis canadensis Ferguson, 1959: NA

Cypridopsis circinata Brady, 1910: AT

Cypridopsis colorata Rome, 1962: AT

Cypridopsis congenera Sars, 1910: AT

Cypridopsis cunningtoni Sars, 1910: AT

Cypridopsis cypera Tressler, 1937: PAC

Cypridopsis dentatomarginata (Daday, 1902) G.W. Müller 1912: NT, PA, PAC

Potamocypris dentatomarginata Daday, 1902

Cypridopsis dubia Sars, 1903: OL, PA

Cypridopsis ellipsoidalis Rome, 1962: AT

Cypridopsis elongata (Kaufmann, 1900) G.W. Müller 1912: PA

Cypridopsella elongata Kaufmann, 1900 (non sensu Sars 1925; = C. hartwigi)

Syn.: Cypridopsella nicki Lindner, 1920 (fide Meisch 2000)

Syn.: Pionocypris almi Sars, 1925 (fide Meisch 2000)

Cypridopsis exigua Sars, 1903: OL

Cypridopsis flavenscens Sars, 1901: NT

Cypridopsis fuhrmanni Méhes, 1914: NT

Cypridopsis funebris Brady, 1886: OL

Cypridopsis gibba Sars, 1910: AT

Cypridopsis hartwigi G.W. Müller, 1900: PA

Syn.: Pionocypris elongata sensu Sars, 1925 (non Kaufmann, 1900) (fide Meisch 2000)

Syn.: Cypridopsis elongata var. orientalis Bronstein, 1925 (fide Meisch 2000)

Syn.: Cypridopsis orientalis (Bronstein, 1925) Bronstein 1947 (fide Meisch 2000)

Syn.: Cypridopsis norvegica Sywula, 1972 (fide Meisch 2000)

Cypridopsis herpestica Cole, 1965: NA

Cypridopsis howei Ferguson, 1964: NA

Cypridopsis japonica Okubo, 1990: PA

Cypridopsis jolleae Chapman, 1963: AU

Cypridopsis kurilensis Schornikov, 1974: PA

Cypridopsis lacustris Rome, 1962: AT

Cypridopsis longa Rome, 1962: AT

Cypridopsis lusatica Schäfer, 1943: PA

Syn.: Cypridopsis lauta Margalef, 1948 (fide Meisch 2000)

Syn.: Cypridopsis brincki Petkovski, 1963 (fide Meisch 2000)

Syn.: Cypridopsis bamberi Henderson, 1986 (fide Meisch 2000)

Cypridopsis malcolmi Löffler, 1968: AT

Cypridopsis mawenzii Löffler, 1968: AT 
Cypridopsis meruensis Lindroth, 1953: AT

Cypridopsis minima Klie, 1935: AT

Cypridopsis minuta Löffler, 1963: NT

Cypridopsis monodonta Sars, 1910: AT

Cypridopsis musquizensis Tressler, 1954: NA

Cypridopsis niagranensis Furtos, 1936: NT

Cypridopsis nigrovittata Okubo, 1990: OL, PA

Cypridopsis obliquata Sars, 1910: AT

Cypridopsis obscura Sars, 1901: NT

Cypridopsis obstinata Barclay, 1968: AU

Cypridopsis oceanus Allison \& Holden, 1971: PAC

Cypridopsis ovalis Rome, 1962: AT

Cypridopsis palustris Gauthier, 1951: AT

Cypridopsis phantomensis Tressler, 1954: NA

Cypridopsis pinguis Sars, 1901: NT

Cypridopsis potamis Tressler, 1954: NA

Cypridopsis pseudoparva Löffler, 1963: NT

Cypridopsis punctillata Brady, 1907: AT

Cypridopsis pusilla Sars, 1910: AT

Cypridopsis raripila Rome, 1962: AT

Cypridopsis reptans Cole, 1965: NA

Cypridopsis rhomboidea Furtos, 1936: NT

Cypridopsis saetosa Hartmann, 1964: PA

Cypridopsis sarasini Méhes, 1939: PA, PAC

Cypridopsis sbruchniensis Kovalenko, 1976: PA

Cypridopsis serrata Sars, 1910: AT

Cypridopsis sinuata Sars, 1910: AT

Cypridopsis toyensis Tressler, 1954: NA

Cypridopsis tumidula Sars, 1910: AT

Cypridopsis uenoi Brehm, 1933: PA

*Cypridopsis vidua (O.F. Müller, 1776) Brady 1867: AT, AU, NA, NT, OL, PA, PAC

Cypris vidua O.F. Müller, 1776

Syn.: Cypris pincta Straus, 1821 (fide Meisch 2000)

Syn.: Cypris sella Baird, 1846 (fide Klie 1938)

Syn.: Cypridopsis obesa Brady \& Robertson, 1869 (fide Meisch 2000)

Syn.: Cypridopsis chavesi Moniez, 1888 (fide Martens \& Savatenalinton 2011)

Syn.: Cypridopsis viduella Sars, 1895 (fide Martens 2001b)

Syn.: Pionocypris assimilis Sars, 1895 (fide Martens 2001b)

? Syn.: Cypridopsis vidua var. concolor Daday, 1900 (fide Meisch 2000)

? Syn.: Cypridopsis concolor Daday, 1900 (sensu Fuhrmann \& Goth 2011; fide Meisch 2000)

Syn.: Cypridopsis helvetica Kaufmann, 1900 (fide Meisch 2000)

Syn.: Cypridopsella tumida Kaufmann, 1900 (fide Meisch 2000)

? Syn.: Cypridopsis parva G.W. Müller, 1900 (sensu Fuhrmann \& Goth 2011; fide Meisch 2000)

Syn.: Proteocypris globuloides Brady, 1907 (fide Martens 2001b)

Syn.: Cypridopsis pustulosa Furtos, 1933 (fide Benson \& McDonald 1963)

Syn.: Cypridopsis africana Klie, 1935 (fide Martens 2001b)

Nom. nov. pro Cypridopsis intermedia (Sars, 1924) nec C. intermedia (Daday, 1900)

Syn.: Cypridopsis okeechobei Furtos, 1936 (fide Martens \& Savatenalinton 2011)

Syn.: Cypridopsis mariae Rome, 1943 (fide Meisch 2000)

Syn.: Cypridopsis sanctipetri Anichini-Pini, 1968 (fide Pieri et al. 2015) 
Syn.: Cypridopsis parvoides J.M. Martens, 1977 (fide Meisch 2000)

Syn.: Cypridopsis biwaensis Okubo, 2004 (nom. nud., no type material designated; fide Smith et al. 2011)

Cyprinotus Brady, 1886

*Cyprinotus cingalensis Brady, 1886: AT, OL, PAC

Cyprinotus crenatus (Turner, 1893) Turner 1895: NA

Cypris crenata Turner, 1893

Cyprinotus dahli Sars, 1896: AU

Cyprinotus dentatus (Sharpe, 1910) Blake 1931: NA

Cypris (Cyprinotus) dentata Sharpe, 1910

Cyprinotus edwardi McKenzie, 1978: AU

Cyprinotus flavescens Brady, 1898: AU

Cyprinotus inconstans Furtos, 1936: NT

Cyprinotus indicus Battish, 1981: OL

Cyprinotus indica Battish, 1981

Heterocypris indica (Battish, 1981) Savatenalinton \& Martens 2011

Cyprinotus kimberleyensis McKenzie, 1966: AU, OL, PA

Syn.: Cyprinotus setoensis Okubo, 1990 (fide Okubo 2004)

Cyprinotus newmexicoensis Ferguson, 1967: NA

Cyprinotus ohanopecoshensis Ferguson, 1966: NA

Cyprinotus pellucidus (Sharpe, 1897) Sharpe 1918: NA, PA

Cyprinotus pellucida [sic] Sharpe 1897

Cyprinotus scytodus (Dobbin, 1941) Tressler 1947: NA

Cypris (Cyprinotus) scytoda Dobbin, 1941

Cyprinotus sulphureus Blake, 1931: NA

Cyprinotus tenuis Henry, 1923: NA

Cyprinotus uenoi Brehm, 1936: OL, PA

Cyprinotus unispinifera Furtos, 1936: NT

Cypris bispinosa Lucas, 1849: PA

Cypris O.F. Müller, 1776

Cypris busingiziensis Klie, 1938: AT

Cypris debi Deb, 1983: OL (uncertain species)

Cypris decaryi Gauthier, 1933: AT, NT, OL, PA

Syn.: Cypris ravenala Brehm, 1934 (fide Martens \& Behen 1994)

Syn.: Cypris labiata Rome, 1962 (fide Martens 1990b)

Cypris dravidensis Victor \& Fernando, 1979: OL

Nom. nov. pro Cypris globulosa Victor \& Michael, 1975 nec Sharpe, 1910

Cypris elburensis Martens, 1990: AT

Cypris elongata Deb, 1983: OL (uncertain species)

Cypris floridensis Ferguson, 1964: NA

Cypris galefensis Martens, 1990: AT

Cypris globosa Deb, 1983: OL (uncertain species)

Cypris granulata Daday, 1898: AT, AU, NA, OL, PA

Syn.: Cypris subglobosa Sowerby, 1840 (fide Klie 1932; records from the Recent only, see Taxonomic notes)

Cypris latissima (G.W. Müller, 1898) G.W. Müller 1912: AT

Eurycypris latissima G.W. Müller, 1898

Syn.: Eurycypris neumanni G.W. Müller, 1900 (fide Gauthier 1939)

Syn.: Cypris inflata Daday, 1910 (fide Martens 1990b)

Syn.: Cypris connica Lowndes, 1936 (fide Gauthier 1939) 
Cypris maculosa Bronstein, 1925: PA

*Cypris pubera O.F. Müller, 1776: AU, NA, NT, PA

Syn.: Cypris pubera var. triaculeata Daday, 1892 syn. nov.

Syn.: Cypris pubera var. polyacantha Daday, 1892 syn. nov.

Syn.: Cypris pubera var. anacantha Daday, 1900 syn. nov.

Syn.: Cypris pubera var. monacantha Daday, 1900 syn. nov.

Syn.: Cypris pubera var. diacantha Daday, 1900 syn. nov.

Syn.: Cypris pubera var. triacantha Daday, 1900 syn. nov.

Syn.: Cypris triaculeata (Daday, 1892) Fuhrmann \& Pietrzeniuk, 1990 syn. nov.

Cypris puberoides Vávra, 1897: AT

Cypris striata (Jurine, 1820) Desmarest, 1825: PA

Monoculus striatus Jurine, 1820

Cyprois Zenker, 1854

*Cyprois marginata (Straus, 1821) Sars, 1890: NA, PA

Cypris marginata Straus, 1821

Syn.: Cyprois dispar Fischer, 1851 (non Chyzer, 1858; fide Klie 1938a)

Syn.: Cyprois flava Zaddach, 1844 (fide Klie 1938a)

Cyprois occidentalis Sars, 1926: NA, PA

Cytheridella Daday, 1905

Syn.: Onychocythere Tressler, 1939 (fide Pinto \& Sanguinetti 1962)

Cytheridella americana (Furtos, 1936) Danielopol 1981: NT

Metacypris americana Furtos, 1936

Cytheridella argentinensis (Ferguson, 1967) Danielopol 1981: NT

Gomphocythere argentinensis Ferguson, 1967

Cytheridella boldi Purper, 1974: NT

Cytheridella damasi Klie, 1944: AT

Syn.: Cytheridella chariessa Rome, 1977 (fide Karanovic 2009)

*Cytheridella ilosvayi Daday, 1905: NA, NT

Syn.: Metacypris ometepensis Swain \& Gilby, 1965 (fide Martens \& Behen 1994)

Syn.: Onychocythere alosa Tressler, 1939 (fide Cohuo et al. 2017)

Cytheridella monodi Klie, 1936: AT

Cytheridella tepida Victor, 1987: AT

Cytherissa Sars, 1925

Cytherissa attenuata Mazepova, 1984: PA

Cytherissa attenuata attenuata Mazepova, 1984

Cytherissa attenuata minor Mazepova, 1990

Cytherissa bisetosa Mazepova, 1984: PA

Cytherissa burchani Mazepova, 1990: PA

Cytherissa calva Mazepova, 1990: PA

Cytherissa compta Mazepova, 1990: PA

Cytherissa crepera Mazepova, 1990: PA

Cytherissa crepera crepera Mazepova, 1990

Cytherissa crepera angustimarginata Mazepova, 1990

Cytherissa crepera magna Mazepova, 1990

Cytherissa cymbulata Mazepova, 1984: PA

Cytherissa cytheriformis Bronstein, 1947: PA

Cytherissa derupta Mazepova, 1984: PA

Cytherissa dextima Mazepova, 1990: PA

Cytherissa donquixotei Mazepova, 1990: PA 
Cytherissa dubitabilis (Bronstein, 1947) Martens \& Savatenalinton 2011: PA

Cytherissa lacustris var. dubitabilis Bronstein, 1947

Cytherissa elongata Bronstein, 1947: PA

Cytherissa elongata elongata Bronstein, 1947

Cytherissa elongata sorensis Mazepova, 1990

Cytherissa excelsa Mazepova, 1990: PA

Cytherissa excelsiformis Mazepova, 1990: PA

Cytherissa florensovi Mazepova, 1990: PA

Cytherissa fuscata Bronstein, 1947: PA

Cytherissa glomerata Mazepova, 1990: PA

Cytherissa golyschkinae Mazepova, 1990: PA

Cytherissa inormis Faradzhev, 1968: PA

Cytherissa interposita Bronstein, 1947: PA

Cytherissa interposita interposita Bronstein, 1947

Cytherissa interposita ushkani Mazepova, 1990

*Cytherissa lacustris (Sars, 1863) Sars, 1925: NA, PA

Cythere lacustris Sars, 1863

Syn.: Acanthopus resistans Vernet, 1878 (fide Kaufmann 1896)

Syn.: Cytherissa lacustris baikalensis Bronstein, 1947 (fide Martens \& Savatenalinton 2011)

Cytherissa lata Bronstein, 1930: PA

Cytherissa latirecta Mazepova, 1985: PA

Cytherissa latiundata Mazepova, 1985: PA

Cytherissa microexsculpta Mazepova, 1984: PA

Cytherissa mirabilis Bronstein, 1947: PA

Cytherissa multipora Mazepova, 1984: PA

Cytherissa nana Mazepova, 1984: PA

Cytherissa neobaicalensis Mazepova, 1990: PA

Nom. nov. pro C. baicalensis Mazepova, 1984 nec C. lacustris baikalensis Bronstein, 1947

Cytherissa obrutshevi Mazepova, 1990: PA

Cytherissa parallela Bronstein, 1947: PA

Cytherissa parva Mazepova, 1984: PA

Cytherissa parva parva Mazepova, 1984

Cytherissa parva confinis Mazepova, 1990

Cytherissa pennata Mazepova, 1990: PA

Cytherissa placida Mazepova, 1990: PA

Cytherissa plena Mazepova, 1985: PA

Cytherissa pterygota Bronstein, 1947: PA

Cytherissa puschkarevi Mazepova, 1990: PA

Cytherissa pusilla Mazepova, 1985: PA

Cytherissa sernovi Bronstein, 1930: PA

Cytherissa sernovi sernovi Bronstein, 1930

Cytherissa sernovi insularis Mazepova, 1990

Cytherissa sernovi ovata Bronstein, 1947

Cytherissa sinistra Mazepova, 1984: PA

Cytherissa sinistrodentata Bronstein, 1930: PA

Cytherissa tenella Mazepova, 1984: PA

Cytherissa triangulata Bronstein, 1947: PA

Cytherissa truncata Bronstein, 1930: PA

Cytherissa tuberculata Bronstein, 1930: PA

Cytherissa tuberculata tuberculata Bronstein, 1930 
Cytherissa tuberculata anisoptera Mazepova, 1990

Cytherissa uvaeformis Mazepova, 1990: PA

Cytherissa verrucosa Mazepova, 1990: PA

Cytherois G.W. Müller, 1884

Type species: Cytherois virens G.W. Müller, 1884 (marine species)

Cytherois arenicola Klie, 1929: PA

Cytherois stephanidesi Klie, 1938: PA

Cytheromorpha Hirschmann, 1909

*Cytheromorpha fuscata (Brady, 1869) Hirschmann 1912: NA, PA

Cythere fuscata Brady, 1869

Cytherura Sars, 1866

*Cytherura gibba (O.F. Müller, 1785) Sars 1866: PA

Cythere gibba O.F. Müller, 1785

Cytherura gibbera (O.F. Müller, 1785) Sars 1866: PA

Cythere gibbera O.F. Müller, 1785

Cytherura propinqua Brady \& Robertson, 1870: PA

Dactylocythere Hart, 1962

Dactylocythere amicula Hart \& Hart, 1966: NA

Dactylocythere amphiakis Hart \& Hart, 1966: NA

Dactylocythere apheles Hobbs \& Walton, 1976: NA

Dactylocythere arcuata (Hart \& Hobbs, 1961) Hart 1962: NA

Entocythere arcuata Hart \& Hobbs, 1961

Dactylocythere astraphes Hobbs \& Walton, 1977: NA

Dactylocythere banana Hart \& Hart, 1971: NA

Dactylocythere brachydactylus Hobbs \& Walton, 1976: NA

Dactylocythere brachystrix Hobbs \& Walton, 1966: NA

Dactylocythere chalaza (Hobbs \& Walton, 1962) Hart 1962: NA

Entocythere chalaza Hobbs \& Walton, 1962

Dactylocythere charadra Hobbs III, 1971: NA

Dactylocythere chelomata (Crawford, 1961) Hart 1962: NA

Entocythere chelomata Crawford, 1961

Dactylocythere coloholca Hobbs \& Hobbs, 1970: NA

Dactylocythere cooperorum Hobbs \& Walton, 1968: NA

Dactylocythere corvus Hobbs \& Walton, 1977: NA

Dactylocythere crawfordi Hart, 1965: NA

Dactylocythere crena Hobbs \& Walton, 1975: NA

Dactylocythere cryptoteresis Hobbs \& Peters, 1993: NA

Dactylocythere daphnioides (Hobbs, 1955) Hart 1962: NA

Entocythere daphnioides Hobbs, 1955

Dactylocythere demissa Hobbs \& Walton, 1976: NA

Dactylocythere enoploholca Hobbs \& Walton, 1970: NA

Dactylocythere exoura Hart \& Hart, 1966: NA

Dactylocythere falcata (Hobbs \& Walton, 1961) Hart 1962: NA

Entocythere falcata Hobbs \& Walton, 1961

Dactylocythere guyandottae Hobbs \& Peters, 1991: NA

Dactylocythere isabelae Hobbs \& Peters, 1977: NA

Dactylocythere jeanae Hobbs, 1967: NA

Dactylocythere koloura Hart \& Hart, 1971: NA

Dactylocythere lepta Hobbs \& Peters, 1991: NA 
Dactylocythere leptophylax (Crawford, 1961) Hart 1962: NA

Entocythere leptophylax Craford, 1961

Dactylocythere macroholca Hobbs \& Hobbs, 1970: NA

Dactylocythere mecoscapha (Hobbs \& Walton, 1960) Hart 1962: NA

Entocythere mecoscapha Hobbs \& Walton, 1960

Dactylocythere megadactylus Hart \& Hart, 1971: NA

Dactylocythere myura Hobbs \& Walton, 1970: NA

Dactylocythere pachysphyrata Hobbs \& Walton, 1966: NA

Dactylocythere peedeensis Hobbs \& Peters, 1977: NA

Dactylocythere phoxa Hobbs, 1967: NA

Dactylocythere prinsi Hobbs \& Walton, 1968: NA

Dactylocythere prionata (Hart \& Hobbs, 1961) Hart 1962: NA

Entocythere prionata Hart \& Hobbs, 1961

Dactylocythere prominula Hobbs \& Walton, 1977: NA

Dactylocythere pughae Hobbs \& Hobbs, 1970: NA

Dactylocythere pygidion Hobbs \& Peters, 1991: NA

*Dactylocythere runki (Hobbs, 1955) Hart 1962: NA

Entocythere runki Hobbs, 1955

Dactylocythere sandbergi Hart \& Hart, 1971: NA

Dactylocythere scissura Hobbs \& Walton, 1975: NA

Dactylocythere scotos Norden \& Norden, 1985: NA

Dactylocythere speira Hart \& Hart, 1971: NA

Dactylocythere spinata Hobbs \& Walton, 1970: NA

Dactylocythere spinescens Hobbs \& Walton, 1977: NA

Dactylocythere steevesi (Hart \& Hobbs, 1961) Hart 1962: NA

Entocythere steevesi Hart \& Hobbs, 1961

Dactylocythere striophylax (Crawford, 1959) Hart 1962: NA

Entocythere striophylax Crawford, 1959

Dactylocythere susanae Hobbs III, 1971: NA

Dactylocythere suteri (Crawford, 1959) Hart 1962: NA

Entocythere suteri Crawford, 1959

Dactylocythere ungulata (Hart \& Hobbs, 1961) Hart 1962: NA

Entocythere ungulata Hart \& Hobbs, 1961

Dactylocythere xystroides Hobbs \& Walton, 1963: NA

Danielocandona Broodbakker, 1983

*Danielocandona lieshoutae Broodbakker, 1983: NT

Darwinula Brady \& Robertson, 1885

Syn.: Polycheles Brady \& Robertson, 1870 (fide Rossetti \& Martens 1998)

Syn.: Darwinella Brady \& Robertson, 1872 (fide Rossetti \& Martens 1998)

*Darwinula stevensoni (Brady \& Robertson, 1870) Brady \& Robertson, 1885: AT, AU, NA, NT, OL, PA Polycheles stevensoni Brady \& Robertson, 1870

Syn.: Argilloecia aurea Brady \& Robertson, 1870 (fide Rossetti \& Martens 1998)

Syn.: Darwinula improvisa Turner, 1895 (fide Rossetti \& Martens 1998)

Syn.: Darwinula protracta Rome, 1953 (fide Rossetti \& Martens 1998)

Syn.: Darwinula variabilis Tagliasacchi, 1968 (fide Rossetti \& Martens 1998)

Syn.: Darwinula sphenoides Rome, 1977 (fide Rossetti \& Martens 1998)

Deminutiocandona Karanovic, 2003

Deminutiocandona aenigma Karanovic, 2007: AU

Deminutiocandona aporia Karanovic, 2007: AU 
Deminutiocandona apposita Karanovic, 2007: AU

Deminutiocandona atope Karanovic, 2007: AU

Deminutiocandona bicauda Karanovic, 2007: AU

*Deminutiocandona mica Karanovic, 2003: AU

Deminutiocandona murrayi Karanovic, 2007: AU

Deminutiocandona neara Karanovic, 2007: AU

Deminutiocandona quasimica Karanovic, 2007: AU

Deminutiocandona stomachosa Karanovic, 2007: AU

Dentocypria Savatenalinton, 2017

Dentocypria aequiloba Savatenalinton, 2017: OL

Dentocypria chantaranothaii Savatenalinton, 2017: OL

Dentocypria mesquitai Savatenalinton, 2017: OL

Dentocypria smithi Savatenalinton, 2017: OL

Diacypris Herbst, 1961

Diacypris compacta (Herbst, 1958) Herbst, 1961: AU

Pseudocypris compacta Herbst, 1958

Diacypris dictyote DeDeckker, 1981: AU

*Diacypris dietzi (Herbst, 1958) Herbst, 1961: AU

Pseudocypris dietzi Herbst, 1958

Diacypris fodiens (Herbst, 1958) Herbst, 1961: AU

Pseudocypris fodiens Herbst, 1958

Diacypris occidentalis McKenzie, 1978: AU

Diacypris paracompacta McKenzie, 1978: AU

Diacypris parva Hartmann, 1978: AU

Diacypris phoxe De Deckker, 1981: AU

Diacypris spinosa De Deckker, 1981: AU

Diacypris whitei (Herbst, 1958) Herbst, 1961: AU

Pseudocypris whitei Herbst, 1958

Diaphanocypris Würdig \& Pinto, 1990

*Diaphanocypris meridana (Furtos, 1936) Würdig \& Pinto 1990: NA, NT Herpetocypris meridana Furtos, 1936

Syn.: Dolerocypris sagitta Klie, 1939 (fide Würdig \& Pinto 1990)

Syn.: Herpetocypris bonettoi Ferguson, 1967 (fide Nagler et al. 2014)

? Syn.: Herpetocypris muhitis Tressler, 1950 (fide Cohuo et al. 2017)

Diaphanocypris pedroensis (Tressler, 1950) Nagler et al. 2014: NT

Strandesia pedroensis Tressler, 1950

Dolekiella Gidó et al., 2007

*Dolekiella europaea Gidó et al., 2007: PA

Dolerocypria Tressler, 1937

Syn.: Thalassocypris Hartmann, 1955 (fide Keyser 1976)

Dolerocypria convulata Maddocks, 1993: NT

Dolerocypria elongata (Hartmann, 1955) Keyser 1976: NT

Thalassocypris elongata Hartmann, 1955

Dolerocypria ensigera Maddocks, 1992: PAC

Dolerocypria fastigata Keyser, 1976: NA

Dolerocypria heylenae Wouters, 2001: PAC 
Dolerocypria iliffei Maddocks, 2005: PAC

Dolerocypria mukaishimensis Okubo, 1980: PA

*Dolerocypria taalensis Tressler, 1937: OL, PA, PAC

Dolerocypris Kaufmann, 1900

*Dolerocypris fasciata (O.F. Müller, 1776) Kaufmann 1900: NA, OL, PA

Cypris fasciata O.F. Müller, 1776

Dolerocypris fasciata fasciata (O.F. Müller, 1776) Kaufmann 1900

Syn.: Monoculus unifasciatus O.F. Müller, 1776 (fide G.W. Müller 1912)

Syn.: Cypris ephippiata Koch, 1837 (fide G.W. Müller 1912)

Syn.: Cypris angustata Sars, 1863 (fide G.W. Müller 1912)

Dolerocypris fasciata nipponensis Okubo, 1972

Dolerocypris ikeyai Smith \& Kamiya, 2006: PA, PAC

Dolerocypris marina Hartmann, 1965: NT

Tanycypris marina (Hartmann, 1965) Broodbakker 1984

Dolerocypris opesta Brehm, 1932: NT

Syn.: Dolerocypris maya Brehm, 1939 (fide Martens \& Behen 1994)

Dolerocypris sinensis Sars, 1903: NA, OL, PA

Dolerocypris sisaketensis Savatenalinton \& Suttajit, 2016: OL

Dolerocypris tenuis (Daday, 1905) G.W. Müller 1912: NT, OL

Eucypris (Eucypris) tenuis Daday, 1905

Donnaldsoncythere Rioja, 1942

Donnaldsoncythere ardis Hobbs \& Walton, 1963: NA

Donnaldsoncythere cayugaensis Hobbs \& Walton, 1966: NA

*Donnaldsoncythere donnaldsonensis (Klie, 1931) Hart 1962: NA

Entocythere donnaldsonensis Klie, 1931

Syn.: Donnaldsoncythere humesi (Hoff, 1943) (fide Hobbs \& Peters 1977)

Syn.: Donnaldsoncythere pennsylvanica (Hart, 1960) (fide Hobbs \& Peters 1977)

Syn.: Donnaldsoncythere hiwasseensis (Hobbs \& Walton, 1961) (fide Hobbs \& Peters 1977)

Syn.: Donnaldsoncythere tuberosa (Hart \& Hobbs, 1961) (fide Hobbs \& Peters 1977)

Syn.: Donnaldsoncythere ileata Hobbs \& Walton, 1963 (fide Hobbs \& Peters 1977)

Syn.: Donnaldsoncythere scalis Hobbs \& Walton, 1963 (fide Hobbs \& Peters 1977)

Donnaldsoncythere leptodrilus Hobbs \& Peters, 1977: NA

Donnaldsoncythere truncata Hobbs \& Walton, 1963: NA

Earicandona Karanovic, 2013

*Earicandona mounchyon Karanovic, 2013: PA

Elachistocythere Hart \& Hart, 1970

* Elachistocythere merista Hart \& Hart, 1970: AU

Elofsonia Wagner, 1957

*Elofsonia baltica (Hirschmann, 1909) Wagner 1957: PA

Loxoconcha baltica Hirschmann, 1909

Elofsonia pusilla (Brady \& Robertson, 1870) Wagner 1957: PA Loxoconcha pusilla Brady \& Robertson, 1870

Elpidium F. Müller, 1880

*Elpidium bromeliarum F. Müller, 1880: NT

Elpidium inaequivalve Danielopol, 1981: NT

Elpidium inaequivalvis Danielopol, 1981 
Elpidium laesslei (Tressler, 1956) Danielopol, 1981: NT

Metacypris laesslei Tressler, 1956

Elpidium maricaoense (Tressler, 1941) Danielopol, 1981: NT

Metacypris maricaoensis Tressler, 1941

Elpidium martensi Danielopol et al., 2014: NT

Elpidium merendonense Pinto \& Jocqué, 2013: NT

Elpidium pintoi Danielopol, 1981: NT

Elpidium purperae Danielopol, 1981: NT

Elpidium purperi Danielopol, 1981

Entocythere Marshall, 1903

*Entocythere cambaria Marshall, 1903: NA

Entocythere claytonhoffi Rioja, 1942: NA, NT

Entocythere costata Hobbs \& Peters, 1977: NA

Entocythere dentata Crawford, 1965: NA

Entocythere dorsorotunda Hoff, 1944: NA

Entocythere elliptica Hoff, 1944: NA

Syn.: Entocythere internotalus Crawford, 1959 (fide Andolshek \& Hobbs 1986)

Entocythere harrisi Peters, 1975: NA

Entocythere illinoisensis Hoff, 1942: NA

Entocythere kanawhaensis Hobbs \& Walton, 1966: NA

Entocythere lepta Hart \& Hart, 1971: NA

Entocythere mexicana Rioja, 1943: NT

Entocythere prisma Andolshek \& Hobbs, 1986: NA

Entocythere reddelli Hobbs \& Walton, 1968: NA

Entocythere ruibali Rioja, 1955: NT

Entocythere tyttha Hobbs \& Hobbs, 1970: NA

Eucyprinotus Sywula, 1972

*Eucyprinotus rostratus (Sywula, 1966) Sywula, 1972: PA

Eucypris rostratus Sywula, 1966

Cypris (Eucypris) Vávra, 1891

Eucypris Vávra, 1891

Syn.: Eucypris (Guaiqueria) Margalef, 1961 (fide Martens \& Behen 1994)

? Syn.: Candocyprinotus Delorme, 1970 (fide Smith \& Horne 2016)

Eucypris afghanistanensis Hartmann, 1964: PA

Syn.: Eucypris gyirongensis Yang, 1982 (fide Mischke et al. 2010)

Eucypris anglica Fox, 1967: PA

Eucypris angulata Hartmann, 1964: PA

Eucypris arcadiae Furtos, 1936: NA

Eucypris areguensis Daday, 1905: NT

Eucypris bayensis Tressler, 1937: OL

Eucypris biharensis Deb, 1984: OL

Eucypris bispinosa (Victor \& Michael, 1975) Victor \& Fernando 1979b: OL

Cyprinotus bispinosus Victor \& Michael, 1975

Eucypris bronsteini Petkovski, 1959: PA

Eucypris cameronensis Røen, 1956: AT

Eucypris cecryphalium Cusminsky et al., 2005: NT

Eucypris compressa Deb, 1983: OL 
Eucypris crassa (O.F. Müller, 1785) G.W. Müller 1912: NA, PA

Cypris crassa O.F. Müller, 1785

Eucypris crinita (Henry, 1923) Chapman 1967: AU

Cypris crinita Henry, 1923

Eucypris domestica Löffler, 1968: AT

Eucypris elliptica (Baird, 1846) Daday 1900: PA

Cypris elliptica Baird, 1846

Eucypris ellipticalis Deb, 1983: OL

Eucypris elongata Stephanides, 1937: PA

Eucypris fabaeformis Daday, 1900: PA, PAC

Eucypris gomti Deb, 1983: OL

Eucypris hamadanensis Hartmann, 1964: PA

Eucypris heinrichi Diebel \& Pietrzeniuk, 1978: PA

Eucypris himani Deb, 1983: OL

Eucypris illyrica Klie, 1937: PA

Eucypris indica Deb, 1983: OL

Eucypris inequalis Deb, 1983: OL

Eucypris kerkyrensis Stephanides, 1937: PA

Eucypris kurtdiebeli Petkovski \& Keyser, 1997: PA

Eucypris lateraria (King, 1855) Chapman 1967: AU

Cypris lateraria King, 1855

Eucypris lilljeborgi (G.W. Müller, 1900) G.W. Müller 1912: PA

Cypris lilljeborgi G.W. Müller, 1900

Syn.: Eucypris lilljeborgi turcica Hartmann, 1964 (fide Martens \& Savatenalinton 2011)

Eucypris lineata Akatova, 1950: PA

Eucypris mareotica (Fischer, 1855) Martens 1984: PA

Cypris mareotica Fischer, 1855

Syn.: Eucypris inflata (Sars, 1903) (fide Martens 1984)

Syn.: Cypricercus mongolicus Daday, 1909 (fide Rasouli et al. 2016)

Syn.: Eucypris inflata mongolica Bronstein, 1947 (fide Martens 1984)

Eucypris meadensis Gutentag \& Benson, 1962: NA

Eucypris minuta Daday, 1908: PA

Eucypris montelgoni Kiss, 1959: AT

Eucypris moravica Jančařík, 1947: PA

Eucypris munia Deb, 1983: OL

Eucypris noodti Hartmann, 1965: NT

Eucypris opulenta Margalef, 1961: NT

Eucypris (Guaiqueria) opulenta Margalef, 1961

Eucypris orca Tressler, 1937: OL

Eucypris pagasti Schäfer, 1952: PA

Eucypris persica Klie, 1937: PA

Eucypris petkovskii Danielopol, 1965: PA

Eucypris pigra (Fischer, 1851) G.W. Müller, 1912: PA

Cypris pigra Fischer, 1851

Syn.: Cypris tumefacta Brady \& Robertson, 1870 (fide G.W. Müller 1912)

Syn.: Eucypris pigra balcanica Petkovski, 1962 (fide Martens \& Savatenalinton 2011)

Eucypris pratensis Eagar, 1970: AU

Eucypris rava Furtos, 1933: NA

Eucypris rischtanica Schneider, 1963: PA

Eucypris salina Hartmann, 1964: PA 
Eucypris sonia Deb, 1983: OL

Eucypris stephanidesi Petkovski, 1959: PA

Eucypris tarentina Anichini, 1963: PA

Eucypris thomsoni Chapman, 1963: AU

Eucypris tibetana Daday, 1908: PA

Eucypris trajani Sywula, 1968: PA

Eucypris trapezoides Hartmann, 1962: NT

Eucypris ungulata (Moniez, 1891) Gauthier 1928: PA

Cypris ungulata Moniez, 1891

*Eucypris virens (Jurine, 1820) Daday 1900: AT, AU, NA, NT, PA

Monoculus virens Jurine, 1820

Syn.: Cypris tristriata Baird, 1835 (fide Daday 1900)

Syn.: Cypris ventricosa Brady \& Robertson, 1870 (fide G.W. Müller 1912)

Syn.: Cypris helena Moniez, 1887 (fide Daday 1900)

Syn.: Eucypris virens var. acuminata G.W. Müller, 1900 (fide Meisch 2000)

Syn.: Eucypris virens var. media G.W. Müller, 1900 (fide Meisch 2000)

Syn.: Eucypris virens var. obtusa G.W. Müller, 1900 (fide Meisch 2000)

Syn.: Eucypris virens var. latissima Alm, 1914 (fide Martens \& Savatenalinton 2011)

Syn.: Eucypris virens var. ruidaschti Akatova, 1950 (fide Martens \& Savatenalinton 2011)

Syn.: Eucypris accipitrina Anichini-Pini, 1968 (fide Pieri et al. 2015)

Syn.: Eucypris hieracina Anichini-Pini, 1968 (fide Pieri et al. 2015)

Syn.: Eucypris longisetosa Anichini-Pini, 1968 (fide Pieri et al. 2015)

Syn.: Eucypris molybdena Anichini-Pini, 1968 (fide Pieri et al. 2015)

Syn.: Eucypris sulcitana Anichini-Pini, 1968 (fide Pieri et al. 2015)

Syn.: Eucypris helocrenica Fuhrmann \& Goth, 2011 syn. nov.

Syn.: Eucypris obtusa (G.W. Müller, 1900) Fuhrmann \& Goth 2011 syn. nov.

Eundacypris Martens, 1986

*Eundacypris superba (Sars, 1924) Martens, 1986: AT

Sclerocypris superba Sars, 1924

Fabaeformiscandona Krstić, 1972

Fabaeformiscandona acuminata (Fischer, 1854) Danielopol 1980: NA, PA

Cypris acuminata Fischer, 1854

Fabaeformiscandona aemonae (Klie, 1935) Meisch 1996: PA

Candona aemonae Klie, 1935

Fabaeformiscandona akaina Smith \& Janz, 2008: PA

Fabaeformiscandona alchichica (Cohuo et al., 2017) comb. nov.: NT

Candona alchichica Cohuo et al., 2017

Fabaeformiscandona alexandri (Sywula, 1981) Griffiths 1995: PA

Candona (Eucandona) alexandri Sywula, 1981

Fabaeformiscandona angusta (Ostermeyer, 1937) Meisch 1996: PA

Candona angusta Ostermeyer, 1937

Fabaeformiscandona balatonica (Daday, 1894) Griffiths \& Mount 1993: NA, PA

Candona balatonica Daday, 1894

Syn.: Candona reniformis Hartwig, 1900 (fide Meisch 2000)

Fabaeformiscandona biangulata (Hoff, 1942) Karanovic 2006: NA

Candona biangulata Hoff, 1942

Syn.: Candona orangeburgensis Ferguson, 1958 (fide Karanovic 2006)

Fabaeformiscandona bilobata (Klie, 1938) Meisch 1996: PA

Candona bilobata Klie, 1938 
Fabaeformiscandona bilobatoides (Löffler, 1961) Meisch 1996: PA

Candona bilobatoides Löffler, 1961

Fabaeformiscandona breuili (Paris, 1920) Meisch 2000: PA

Candona breuili Paris, 1920

Syn.: Candona hertzogi Klie, 1937 (fide Meisch 2000)

Syn.: Candona spelaea Klie, 1941 (fide Meisch 2000)

Syn.: Candona hertzogi beskidana Sywula, 1974 (fide Meisch 2000)

Fabaeformiscandona brevicornis (Klie, 1925) Meisch 1996: PA

Candona brevicornis Klie, 1925

Syn.: Candona (Typhlocypris) limnocrenica Sywula, 1971 (fide Meisch 2000)

Fabaeformiscandona brisiaca (Klie, 1938) Meisch 1996: PA

Candona brisiaca Klie, 1938

Fabaeformiscandona caucasica (Bronstein, 1928) Wilkinson et al. 2005: PA

Syn.: Candona elpatiewskyi var. caucasica Bronstein, 1928 (fide Bronstein 1947)

Fabaeformiscandona caudata (Kaufmann, 1900) Danielopol et al.1988: NA, PA

Candona caudata Kaufmann, 1900

Syn.: Candona elongata Brady \& Norman, 1889 (unused older synonym; fide Klie 1938a)

Syn.: Fabaeformiscandona caudata var. ciliata (Furtos, 1935) (fide Karanovic 2006)

Candona caudata var. ciliata Furtos, 1935

Syn.: Fabaeformiscandona caudata occidentalis (Dobbin, 1941) (fide Karanovic 2006)

Candona caudata occidentalis Dobbin, 1941

Fabaeformiscandona condylea Smith \& Janz, 2008: PA

Fabaeformiscandona danielopoli Yin \& Martens, 1997: PA

Fabaeformiscandona distincta (Furtos, 1933) Martens \& Savatenalinton 2011: NA

Candona distincta Furtos, 1933

Fabaeformiscandona dolabella Smith \& Janz, 2008: PA

Fabaeformiscandona dorsobiconcava (Bronstein, 1947) Wilkinson et al. 2005: PA

Candona dorsobiconcava Bronstein, 1947

*Fabaeformiscandona fabaeformis (Fischer, 1851) Danielopol 1973: OL, PA

Cypris fabaeformis Fischer, 1851

Syn.: Candona diaphana Brady \& Robertson, 1870 (fide Klie 1938a)

Syn.: Candona bradyi Hartwig, 1898 (fide Klie 1938a)

? Syn.: Candona fuhrmanni Thiébaud, 1908 (fide Meisch 2000)

Fabaeformiscandona fabella (Nüchterlein, 1969) Griffiths \& Evans 1995: PA

Candona fabella Nüchterlein, 1969

Syn.: Candona (Eucandona) slesiniaca Sywula, 1974 (fide Meisch 2000)

Fabaeformiscandona forma (Karanovic, 1999) Martens \& Savatenalinton 2011: PA

Eucandona forma Karanovic, 1999

Fabaeformiscandona fragilis (Hartwig, 1898) Danielopol 1980: PA

Candona fragilis Hartwig, 1898

Fabaeformiscandona groenlandica (Brehm, 1911) Martens \& Savatenalinton 2011: NA, PA

Candona groenlandica Brehm, 1911

Fabaeformiscandona gyirongensis (Huang, 1982) Wrozyna et al. 2009: PA

Candona gyirongensis Huang, 1982

Fabaeformiscandona harmsworthi (Scott, 1899) Griffiths 1996: NA, PA

Candona harmsworthi Scott, 1899

Syn.: Candona rectangulata Alm, 1914 (fide Namiotko et al. 2009)

Fabaeformiscandona holzkampfi (Hartwig, 1900) Wouters 1989: OL, PA

Candona holzkampfi Hartwig, 1900

Syn.: Eucandona csikii pannonica Daday, 1903 (fide Bronstein 1947) 
MEISCH C. et al., Global checklist of extant non-marine Ostracoda

Fabaeformiscandona hyalina (Brady \& Robertson, 1870) Danielopol 1980: NA, PA

Candona hyalina Brady \& Robertson, 1870

Fabaeformiscandona koreana Karanovic \& Lee, 2012: PA

Fabaeformiscandona krstici (Petkovski, 1969) Krstić 1979: PA

Candona krstici Petkovski, 1969

Fabaeformiscandona kushiroensis Hiruta \& Hiruta, 2015: PA

Fabaeformiscandona lapponica (Ekman, 1908) Griffiths 1995: NA, PA

Candona lapponica Ekman, 1908

Fabaeformiscandona lapponica var. arctica (Alm, 1914) Wetterich et al. 2008a

Candona lapponica var. arctica Alm, 1914

Fabaeformiscandona latens (Klie, 1940) Danielopol 1980: PA

Candona latens Klie, 1940

Fabaeformiscandona levanderi (Hirschmann, 1912) Griffiths 1995: PA

Candona levanderi Hirschmann, 1912

Syn.: ? Candona elongata Holmes, 1937 (fide Meisch 2000)

Fabaeformiscandona myllaina Smith \& Kamiya, 2007: PA

Fabaeformiscandona nishinoae Smith \& Janz, 2008: PA

Fabaeformiscandona obtusa (Bronstein, 1947) Martens \& Savatenalinton 2011: NA, NT, PA

Candona obtusa Bronstein, 1947

Syn.: Candona patzcuaro Tressler, 1954 (fide Karanovic 2006)

Syn.: Candona hipolitensis Tressler, 1954 (fide Delorme 1970)

Syn.: Candona michoa Tressler, 1954 (fide Karanovic 2006)

Syn.: Candona rawsoni Tressler, 1957 (fide Delorme 1970)

Syn.: Candona nyensis Gutentag \& Benson, 1962 (fide Delorme 1970)

Syn.: Candona swaini Staplin, 1963 (fide Delorme 1970)

Syn.: Candona swaini sappaensis Staplin, 1963 (fide Delorme 1970)

Syn.: Candona verretensis Leroy, 1964 (fide Delorme 1970)

Fabaeformiscandona okuboi Smith \& Janz, 2008: PA

Fabaeformiscandona paterea Smith \& Janz, 2008: PA

Fabaeformiscandona pedana Smith \& Janz, 2008: PA

Fabaeformiscandona pedata (Alm, 1914) Wetterich et al. 2008b: PA

Candona pedata Alm, 1914

Fabaeformiscandona pennaki Marmonier \& Ward, 1990: NA

Fabaeformiscandona protzi (Hartwig, 1898) Danielopol et al. 1988: NA, PA

Candona protzi Hartwig, 1898

Fabaeformiscandona pyrenaica (Margalef, 1952) Baltanás et al. 1996: PA

Candona pyrenaica Margalef, 1952

Fabaeformiscandona siliquosa (Brady, 1910) Griffiths \& Evans 1995: PA

Candona siliquosa Brady, 1910

Fabaeformiscandona subacuta (Yang, 1982) Schornikov \& Trebukhova 2001: AU, NT, OL, PA

Candona subacuta Yang, 1982

Syn.: Candona sangganheensis Huang, 1985 (fide Escrivá et al. 2012)

Syn.: Candona japonica Okubo, 1990 (fide Escrivá et al. 2012)

Fabaeformiscandona svetozari (Petkovski \& Karanovic, 2004) Martens \& Savatenalinton 2011: PA

Eucandona svetozari Petkovski \& Karanovic, 2004

Fabaeformiscandona thienemanni (Klie, 1932) comb. nov.: OL

Candona thienemanni Klie, 1932

Fabaeformiscandona tora Smith \& Kamiya, 2007: PA

Fabaeformiscandona tricicatricosa (Diebel \& Pietrzeniuk, 1969) Griffiths 1995: PA

Candona tricicatricosa Diebel \& Pietrzeniuk, 1969

Syn.: Candona lozeki Absolon, 1973 (fide Danielopol et al. 2015) 
Fabaeformiscandona tyrolensis (Löffler, 1963) Meisch 2000: PA

Candona tyrolensis Löffler, 1963

Fabaeformiscandona velifera Smith \& Janz, 2008: PA

Fabaeformiscandona wegelini (Petkovski, 1962) Danielopol 1980: NA, PA

Candona wegelini Petkovski, 1962

Fabaeformiscandona yajimae Smith \& Janz, 2008: PA

Frambocythere Colin, 1981

Type species: Frambocythere tumiensis (Helmdach, 1978) Colin 1981 (fossil species)

Frambocythere relicta Smith et al., 2012: PA

Galolimnocythere Schornikov, 1973

Limnocythere (Galolimnocythere) Schornikov, 1973

Galolimnocythere baikaliensis (Martens \& Mazepova, 1992) Martens 1996: PA

Limnocythere baikaliensis Martens \& Mazepova, 1992

* Galolimnocythere sarsi (Schornikov, 1973) Martens 1996: PA

Limnocythere (Galolimnocythere) sarsi Schornikov, 1973

Geocythere acuta Hart \& Hart, 1971: NA

Geocythere Hart, 1962

*Geocythere geophila (Hart, 1959) Hart 1962: NA

Entocythere geophila Hart, 1959

Geocythere gyralea Hart, 1965: NA

Geocythere nessoides Hobbs \& Hobbs, 1970: NA

Globocypris Klie, 1939

*Globocypris trisetosa Klie, 1939: AT

Gomphocythere Sars, 1924

Gomphocythere aethiopis Rome, 1970: AT

Gomphocythere alata Rome, 1962: AT

Gomphocythere angulata Lowndes, 1932: AT

Gomphocythere angusta Klie, 1939: AT

Gomphocythere australica Hussainy, 1969: AU

Gomphocythere besni Külköylüoğlu et al., 2015: PA

Gomphocythere capensis G.W. Müller, 1914: AT

Syn.: Gomphocythere expansa Sars, 1924 (fide Martens 2001b)

Gomphocythere coheni Park \& Martens, 2001: AT

Gomphocythere cristata Rome, 1962: AT

Gomphocythere curta Rome, 1962: AT

Gomphocythere downingi Park \& Martens, 2001: AT

Gomphocythere duffi (Hornibrook, 1955) Barclay 1968: AU

Limnicythere duffi Hornibrook, 1955

Gomphocythere emrysi Martens, 2003: AT

Gomphocythere huwi Martens, 2003: AT

Gomphocythere irvinei Martens, 2003: AT

Gomphocythere lenis Rome, 1962: AT

Gomphocythere lisae Martens, 2003: AT

* Gomphocythere obtusata Sars, 1910: AT

Gomphocythere ortali Martens, 1993: PA

Gomphocythere parcedilatata Rome, 1977: AT

Gomphocythere piriformis Martens, 2003: AT 
Gomphocythere problematica (Brehm, 1932) Brehm 1939: AU

Limnocythere problematica Brehm, 1932

Gomphocythere simplex Rome, 1962: AT

Gomphocythere wilsoni Park \& Martens, 2001: AT

Gomphocythere woutersi Park \& Martens, 2001: AT

Gomphodella De Deckker, 1981

Gomphodella alexanderi Karanovic \& Humphreys, 2014: AU

Gomphodella aura Karanovic, 2009: AU

Gomphodella glomerosa Karanovic, 2006: AU

Gomphodella hirsuta Karanovic, 2006: AU

*Gomphodella maia De Deckker, 1981: AU

Gomphodella martensi Karanovic, 2009: AU

Gomphodella pilbarensis Karanovic, 2009: AU

Gomphodella quasihirsuta Karanovic, 2009: AU

Gomphodella yandii Karanovic, 2006: AU

Gurayacypris Battish, 1987

*Gurayacypris kangraensis Battish, 1987: OL

Hancockcandonopsis Karanovic, 2018

*Hancockcandonopsis inachos Karanovic, 2018: AU

Hancockcandonopsis io Karanovic, 2018: AU

Hancockcandonopsis tamworthi Karanovic, 2018: AU

Hansacypris Wouters, 1984

Type species: Hansacypris aspera Wouters, 1984: PAC (marine species)

Hansacypris galapagosensis Maddocks, 1992: PAC

Hansacypris glabra Wouters, 1984: PAC

Hansacypris motuporensis Wouters, 2001: PAC

Hansacypris natans (Hartmann, 1984) Wouters 1986: PAC

Renaudcypris natans Hartmann, 1984

Harpagocythere Hobbs III, 1965

Harpagocythere baileyi Hobbs \& Peters, 1977: NA

*Harpagocythere georgiae Hobbs III, 1965: NA

Harpagocythere tertius Hobbs \& Walton, 1968: NA

Hartiella Danielopol, 1971

*Hartiella dudichi (Klie, 1938) Danielopol 1971: PA

Sphaeromicola dudichi Klie, 1938

Hartocythere Hobbs III, 1970

*Hartocythere torreya (Hart, 1959) Hobbs III 1970: NA

Entocythere torreya Hart, 1959

Hemicypris Sars, 1903

Hemicypris anomala (Klie, 1938) Purper \& Würdig-Maciel 1974: NT, OL, PA

Heterocypris anomala Klie, 1938

Hemicypis arorai Mannikeri \& Vaidya, 1990: OL

Hemicypris aurita (Klie, 1939) Purper \& Würdig-Maciel 1974: AT, NT

Heterocypris aurita Klie, 1939 
Hemicypris bairdi Martens \& Wouters, 1985: OL

Nom. nov. pro Cypris dentatomarginata (Baird, 1859) (partim) nec Cypris dentatomarginata

Baird, 1859 (partim)

Hemicypris barbadensis Broodbakker, 1983: NT, PA

Hemicypris battishi Mannikeri \& Vaidya, 1990: OL

Hemicypris communis (Klie, 1940) Purper \& Würdig-Maciel 1974: NT

Heterocypris communis Klie, 1940

Hemicypris congenera (Vávra, 1897) sensu Daday 1910 nec Vávra, 1897: AT, PA (see Taxonomic notes)

Cyprinotus congener Vávra, 1897 sensu Daday 1910 nec Vávra, 1897

Hemicypris decorata (Daday, 1910) Bate 1970: AT

Cyprinotus decoratus Daday, 1910

Hemicypris dentatomarginata (Baird, 1859) McKenzie 1972: OL, PA

Cypris dentatomarginata Baird, 1859

Hemicypris derweshensis Battish, 1981: OL

Hemicypris dissona Victor \& Fernando, 1976: OL

Hemicypris exigua Broodbakker, 1983: NT, OL

Hemicypris falcata Victor \& Fernando, 1976: OL

Hemicypris fossulata (Vávra, 1897) McKenzie 1966a: AT

Cyprinotus fossulatus Vávra, 1897

Hemicypris fuelleborni (Daday, 1910) Bate 1970: AT

Eucypris fuelleborni Daday, 1910

Hemicypris futunaensis Victor \& Fernando, 1978: OL, PAC

Hemicypris humbertii (Gauthier, 1933) Bate 1970: AT

Cyprinotus humbertii Gauthier, 1933

Hemicypris intermedia (Lindroth, 1953) Bate 1972: AT

Cyprinotus intermedius Lindroth, 1953

Hemicypris inversa (Daday, 1913) Bate 1970: AT, PA

Cyprinotus inversus Daday, 1913

Hemicypris irakensis Al-Da'amy, 2010: PA

Hemicypris kaufmanni (Vávra, 1906) Bate 1970: PA

Cyprinotus kaufmanni Vávra, 1906

Hemicypris kissi Martens, 1984: AT

Nom. nov. pro Hemicypris dentatomarginata Kiss, 1959 nec Baird, 1859

Hemicypris kliei (Lindroth, 1953) Bate 1972: AT

Cyprinotus kliei Lindroth, 1953

Syn.: Hemicypris posterotruncata Bate, 1970 (fide Martens 1984)

Hemicypris largereticulata (Rome, 1969) Freels 1980: AT

Cyprinotus largereticulatus Rome, 1969

Hemicypris levis (Hartmann, 1964) Purper \& Würdig-Maciel 1974: PA

Heterocypris levis Hartmann, 1964

Hemicypris malerkotlaensis Battish, 1981: OL

Hemicypris megalops Sars, 1903: AU, OL, PA

Syn.: Hemicypris kibiensis Okubo, 1990 (fide Okubo 2004)

Hemicypris mizunoi Okubo, 1990: OL, PA

Hemicypris nonstriata (Lindroth, 1953) Bate 1972: AT, PA

Cyprinotus nonstriatus Lindroth, 1953

Hemicypris ovata Sars, 1903: NT, OL, PA

Syn.: Hemicypris nipponica Okubo, 1990 (fide Okubo 2004)

Hemicypris pailensis Battish, 1981: OL

Hemicypris pandei Bhatia \& Singh, 1977: OL 
Hemicypris paucipustulosa Victor \& Fernando, 1981: OL

*Hemicypris pyxidata (Moniez, 1892) Sars 1903a: AU, OL, PA

Cyprinotus pyxidatus Moniez, 1892

Hemicypris rara (Klie, 1940) Purper \& Würdig-Maciel 1974: NT

Heterocypris rara Klie, 1940

Hemicypris reticulata (Klie, 1930) Bate 1970: AT, NT, OL

Heterocypris reticulatus Klie, 1930

Hemicypris salaria (Hartmann, 1962) Purper \& Würdig-Maciel 1974: NT

Heterocypris salaria Hartmann, 1962

Hemicypris stenoglypha (Rome, 1969) Freels 1980: AT

Cyprinotus stenoglyphus Rome, 1969

Hemicypris vulgaris Okubo, 1990: PA

Herpetocyprella Daday, 1909

*Herpetocyprella mongolica Daday, 1909: PA

Herpetocypris Brady \& Norman, 1889

Syn.: Erpetocypris Brady \& Norman, 1889 (fide G.W. Müller 1912)

Syn.: Siphlocandona Brady, 1910 (fide Fox 1964)

Herpetocypris aequalis Chapman, 1934: AU

Herpetocypris brevicaudata Kaufmann, 1900: NA, PA

Syn.: Herpetocypris ghigii Masi, 1932 (fide Gonzalez Mozo et al. 1996)

Syn.: Herpetocypris lenta Rome, 1947 (fide Gonzalez Mozo et al. 1996)

Syn.: Herpetocypris flumendosa Anichini, 1967 (fide Gonzalez Mozo et al. 1996)

Herpetocypris chevreuxi (Sars, 1896) G.W. Müller 1912: NA, NT, PA

Stenocypris chevreuxi Sars, 1896

Syn.: Cypris hessei Keilhack, 1911 (fide Meisch 2000)

Syn.: Herpetocypris tarnogradskyi Bronstein, 1925 (fide Meisch 2000)

Syn.: Herpetocypris agilis Rome, 1954 (fide Gonzalez Mozo et al. 1996)

Syn.: Herpetocypris romei Anichini, 1967 syn. nov.

Syn.: Herpetocypris scoininosa Anichini, 1968 (fide Gonzalez Mozo et al. 1996)

Herpetocypris fontinalis Bronstein, 1928: PA

Herpetocypris helenae G.W. Müller, 1908: AT, NT, PA

Syn.: Cypris intermedia latialis Masi, 1905 (fide Gonzalez Mozo et al. 1996) (unused senior synonym)

Syn.: Herpetocypris palpiger Lowndes, 1932 (fide Meisch 2000)

Syn.: Herpetocypris caerulea Rome, 1954 (fide Meisch 2000)

Syn.: Herpetocypris puteolina Anichini, 1968 (fide Meisch 2000)

Herpetocypris intermedia Kaufmann, 1900: NT, PA

Syn.: Herpetocypris andegavensis Anichini, 1968 (fide Meisch 2000)

Herpetocypris laevissima Henry, 1923: NA

Herpetocypris ludhianensis Battish, 1982: OL

Herpetocypris mateusorum Paulo, 1969: PA

Herpetocypris pattersoni Tressler, 1954: NA

Herpetocypris pectinata Brehm, 1934: NT

Herpetocypris peregrina Croneberg, 1894: PA

*Herpetocypris reptans (Baird, 1835) Brady \& Norman 1889: NA, NT, PA

Cypris reptans Baird, 1835

Syn.: Candonopsis complanata Brady, 1902 (fide Martens 1984)

Syn.: Candona virescens Brady, 1864 (fide Sars 1925) 
Herpetocypris reptans var. curvata Kaufmann, 1900

Herpetocypris smaragdea Daday, 1908: PA

Herpetocypris stewarti Daday, 1908: PA

Herpetocypris syriaca Daday, 1911: PA

Herpetocythere Hart \& Hart, 1967

Herpetocythere acanthoides Hart \& Hart, 1967: AU

*Herpetocythere australensis Hart \& Hart, 1967: AU

Herpetocythere bendora Hart \& Hart, 1967: AU

Herpetocythere gnoma Hart \& Hart, 1967: AU

Herpetocythere labidioides Hart \& Hart, 1967: AU

Herpetocythere mackenziei Hart \& Hart, 1967: AU

Hesperocythere Hart \& Hart, 1967

*Hesperocythere klasteroides Hart \& Hart, 1967: AU

Hesperocythere tallanalla Hart \& Hart, 1967: AU

Hesperocythere xiphoides Hart \& Hart, 1967: AU

Heterocypris Claus, 1892

Syn.: Cyprinotus (Cyprinotoides) Masi, 1925 (partim; fide Martens 1984)

Syn.: Manuelcypris Yoo et al., 2017

Heterocypris affinis Klie, 1930: NT

Heterocypris anitae Battish, 1981: OL

Heterocypris antillensis Broodbakker, 1982: NT

Manuelcypris antillensis (Broodbakker, 1982) Yoo et al. 2017

Heterocypris arorai Battish, 1981: OL

Heterocypris aurea (Sars, 1895) Sars 1924a: AT, NA, OL, PA

Cyprinotus aureus Sars, 1895

Heterocypris auricularis Zhai \& Zhao, 2014: PA

Syn.: Heterocypris sanukiensis Okubo, 2004 (nom. nud., no type material designated) (fide

Zhai \& Zhao 2014)

Heterocypris balnearia (Moniez, 1893) Klie 1939b: PA

Cypris balnearia Moniez, 1893

Heterocypris barbara (Gauthier \& Brehm, 1928) Margalef 1948: PA

Cyprinotus barbarus Gauthier \& Brehm, 1928

Syn.: Cyprinotus barbarus inermis Gauthier, 1928 (fide Martens et al. 2002)

Syn.: Heterocypris turcica Schäfer, 1952 (fide Martens et al. 2002)

Syn.: Heterocypris rostrata Beldescu, 1961 (fide Martens et al. 2002)

Syn.: Heterocypris bulgarica Sywula, 1967 (fide Martens et al. 2002)

Syn.: Heterocypris vitrea Sywula, 1967 (fide Martens et al. 2002)

Syn.: Heterocypris nurriensis Tagliasacchi Masala, 1969 (fide Martens et al. 2002)

Syn.: Heterocypris takedai Okubo, 1973 (fide Martens \& Savatenalinton 2011)

Syn.: Heterocypris erikae Petkovski \& Keyser, 1995 (fide Martens et al. 2002)

Heterocypris bhatiai Battish, 1981: OL

Heterocypris bogotensis Roessler, 1982: NT

Heterocypris bosniaca Petkovski et al., 2000: PA

Heterocypris calva (Rome, 1965) McKenzie 1971: AT

Cyprinotus calvus Rome, 1965

Heterocypris capensis (G.W. Müller, 1908) Sars 1924a: AT

Cyprinotus capensis G.W. Müller, 1908

Heterocypris carolinensis (Ferguson, 1958) Martens \& Savatenalinton 2011: NA

Cyprinotus carolinensis Ferguson, 1958 
Heterocypris chandrai (Arora, 1931) McKenzie 1972: OL

Cyprinotus chandrai Arora, 1931

Heterocypris chetumalensis (Yoo et al., 2017) comb. nov.: NT

Manuelcypris chetumalensis Yoo et al., 2017

Heterocypris ciliata (Thomson, 1879) Eagar 1971: AU

Cypris ciliata Thomson, 1879

Heterocypris cisternina (Furtos, 1936) comb. nov.: NT

Eucypris cisternina Furtos, 1936

Heterocypris congenera (Vávra, 1897) Klie 1933: AT, OL, PA

Cyprinotus congenera Vávra, 1897

Heterocypris crenata (Turner, 1893) Purper \& Würdig-Maciel 1974: NA, OL

Cypris crenata Turner, 1893

Heterocypris dubia Sars, 1910: AT

Heterocypris exigua (Gauthier \& Brehm, 1928) Klie 1938c: PA, AT

Cyprinotus exiguus Gauthier \& Brehm, 1928

Heterocypris favosa Victor \& Fernando, 1980: OL

Nom. nov. pro Heterocypris reticulata (Tressler, 1937) nec Klie, 1930

Heterocypris fluviatilis (Furtos, 1933) Purper \& Würdig-Maciel 1974: NA, OL

Cyprinotus fluviatilis Furtos, 1933

Heterocypris gevgelica Petkovski et al., 2000: PA

Heterocypris giesbrechti (G.W. Müller, 1898) Löffler 1961: AT, OL

Cyprinotus giesbrechtii G.W. Müller, 1898

Syn.: Cyprinotus incongruens rosea Masi, 1905 (fide Martens et al. 2002)

Syn.: Cypris gunningi Methuen, 1910 (fide Martens et al. 2002)

Syn.: Cyprinotus hesperidum Masi, 1925 (fide Martens et al. 2002)

Syn.: Cyprinotus imus Gauthier, 1934 (fide Martens et al. 2002)

Syn.: Cyprinotus rotondus Rome, 1969 (fide Martens et al. 2002)

Heterocypris gillensis Battish, 1981: OL

Heterocypris glauca (Furtos, 1933) Purper \& Würdig-Maciel 1974: NA

Cyprinotus glaucus Furtos, 1933

Heterocypris gregaria (Skogsberg, 1917) Freels 1980: AU, OL, PA

Cypris (Cyprinotus) gregarius Skogsberg, 1917

Syn.: Cyprinotus dentatomarginatus Sars, 1889 (fide Martens \& Wouters 1985)

Heterocypris humilis (Lindroth, 1953) Purper \& Würdig-Maciel 1974: AT

Cyprinotus humilis Lindroth, 1953

Heterocypris hyalina Klie, 1930: NT

*Heterocypris incongruens (Ramdohr, 1808) Claus 1892: AT, AU, NA, NT, OL, PA, PAC

Cypris incongruens Ramdohr, 1808

Syn.: Monoculus conchaceus Linné, 1758 (unused older synonym) (fide Alm 1915)

Syn.: Monoculus ruber Jurine, 1820 (fide G.W. Müller 1912)

Syn.: Monoculus aurantius Jurine, 1820 (fide G.W. Müller 1912)

Syn.: Cypris fusca Straus, 1821 (fide G.W. Müller 1912)

Syn.: Heterocypris incongruens elongata (Kaufmann, 1900) (fide Martens et al. 2002)

Cypris incongruens elongata Kaufmann, 1900

Syn.: Cyprinotus hertwigi Lindner, 1922 (fide Martens et al. 2002)

Syn.: Heterocypris obliqua Lowndes, 1936 (fide Martens \& Savatenalinton 2011)

Syn.: Heterocypris incongruens attenuata (Gauthier, 1938) (fide Martens et al. 2002)

Cyprinotus incongruens attenuata Gauthier, 1938

Heterocypris kervillei (Daday, 1911) Schäfer 1952: PA

Eucypris kervillei Daday, 1911 
Heterocypris leana (Sars, 1896) De Deckker 1979b: AU

Cypris leana Sars, 1896

Heterocypris luzonensis Neale, 1981: OL

Heterocypris makua (Tressler, 1937) Victor \& Fernando 1980: OL, PAC

Cyprinotus makua Tressler, 1937

Heterocypris malini (Deb, 1983) Martens \& Savatenalinton 2011: OL

Cyprinotus malini Deb, 1983

Heterocypris margaritae Margalef, 1961: NT

Heterocypris monodi (Gauthier, 1938) Martens et al. 2002: AT

Syn.: Cyprinotus symmetricus monodi Gauthier, 1938 (fide Martens et al. 2002)

Heterocypris nicaraguensis Hartmann, 1959: NT

Heterocypris nuda (Victor \& Michael, 1975) Victor \& Fernando 1980: OL

Cyprinotus nudus Victor \& Michael, 1975

Heterocypris oblonga (Sars, 1924) McKenzie 1971: AT

Herpetocypris oblonga Sars, 1924

Heterocypris ovularis (Sars, 1924) McKenzie 1971: AT

Herpetocypris ovularis Sars, 1924

Heterocypris panningi Brehm, 1934: NT

Heterocypris parasimilis Martens, 1994: NT

Nom. nov. pro Heterocypris similis Klie, 1933 nec Wierzejski, 1893

Heterocypris persica (Ghetti, 1972) Petkovski et al. 2000: PA

Cyprinotus persicus Ghetti, 1972

Heterocypris punctata Keyser, 1976: NA, NT

Manuelcypris punctata (Keyser, 1976) Yoo et al. 2017

Heterocypris putei (Furtos, 1936) Victor \& Fernando 1980: NT

Cyprinotus putei Furtos, 1936

Heterocypris reptans (Kaufmann, 1900) Victor \& Fernando 1980: PA

Microcypris reptans Kaufmann, 1900

Syn.: Cypris kaufmanni limbata Masi, 1905 (fide Meisch 1993)

Syn.: Heterocypris brteki Petkovski, 1966 (fide Meisch 1993)

Heterocypris rotundata (Bronstein, 1928) Bronstein 1947: PA, AT

Cyprinotus rotundatus Bronstein, 1928

Syn.: Cyprinotus euplocamus Lowndes, 1931 (fide Martens et al. 2002)

Syn.: Cyprinotus dextrodepressus Moroni, 1961 (fide Pieri et al. 2015)

Heterocypris sabirae Gülen, 1985: PA

Heterocypris salina (Brady, 1868) Klie, 1932: NA, NT, OL, PA

Cypris salina Brady, 1868

Syn.: Cypris prasina Fischer, 1855 (unused senior synonym) (fide G.W. Müller 1912)

Syn.: ? Eucypris palermitana (Fischer, 1855) sensu Daday 1900 (fide G.W. Müller 1912)

Syn.: Cyprinotus fretensis Brady \& Robertson, 1870 (fide Harding 1955)

Syn.: Cypris fragilis Brady, 1902 (fide Martens et al. 2002)

Syn. Cypris nusbaumi Grochmalicki, 1911 (fide Sywula 1974)

Syn.: Cypris lamperti Lindner, 1920 (fide Martens et al. 2002)

Syn.: Cyprinotus inaequivalvis Bronstein, 1928 (fide Petkovski 1964)

Syn.: Cyprinotus sobrinus Masi, 1932 (fide Martens 1984)

Syn.: Cyprinotus maurus Masi, 1932 (fide Martens et al. 2002)

Syn.: Cyprinotus rostratus Lowndes, 1932 (fide Harding 1955)

Syn.: Cyprinotus ichnusae Anichini, 1967 (fide Martens et al. 2002)

Heterocypris sarsi (Brady, 1906) Purper \& Würdig-Maciel 1974: AU

Cyprinotus sarsi Brady, 1906 
Heterocypris similis (Wierzejski, 1893) Purper \& Würdig-Maciel 1974: NT

Cypris (Eucypris) similis Wierzejski, 1893

Heterocypris somalica (Masi, 1925) Klie 1938: AT

Cyprinotus somalicus Masi, 1925

Heterocypris symmetrica (G.W. Müller, 1898) Löffler 1961: AT, NA, NT, PA

Cyprinotus symmetricus G.W. Müller, 1898

Heterocypris syriaca (Daday, 1911) Martens \& Savatenalinton 2011: PA

Cyprinotus syriacus Daday, 1911

Heterocypris tabascena (Yoo et al., 2017) comb. nov.: NT

Manuelcypris tabascena Yoo et al., 2017

Heterocypris tatei (Brady, 1886) De Deckker 1979b: AU

Cypris tatei Brady, 1886

Heterocypris vandouwei (Brehm, 1923) Brehm 1936: PA

Cyprinotus vandouwei Brehm, 1923

Heterocypris vatia De Deckker, 1981: AU

Heterocypris wolffhuegeli (Méhes, 1914) Klie 1930: NT, PAC

Eucypris wolffhuegeli Méhes, 1914

Heterocypris zugmayeri (Brehm, 1914) Löffler 1961: PA

Cyprinotus zugmayeri Brehm, 1914

Hobbsiella Danielopol \& Hart, 1985

*Hobbsiella cirolanae (Rioja, 1951) Danielopol \& Hart 1985: NA

Sphaeromicola cirolanae Rioja, 1951

Hobbsiella coahuiltecae (Hobbs \& Hobbs, 1973) Danielopol \& Hart 1985: NA Sphaeromicola coahuiltecae Hobbs \& Hobbs, 1973

Hobbsiella moria (Hart, 1978) Danielopol \& Hart 1985: NA Sphaeromicola moria Hart, 1978

*Homocypris conoidea Sars, 1924: AT

Homocypris Sars, 1924

Syn.: Homocypris longirostrata Rome, 1965 (fide Martens 2001b)

Humphcypris Martens, 1997

Humphcypris anomala (Lindroth, 1953) Martens 1997: AT

Stenocypris decipiens anomala Lindroth, 1953

Syn.: Stenocypris decipiens mawenzii Löffler, 1968 (fide Martens 1997)

Humphcypris brevisetosa (Lowndes, 1932) Martens, 1997: AT

Stenocypris brevisetosa Lowndes, 1932

Syn.: Stenocypris parva Lowndes, 1932 (= juvenile, fide Martens 1997)

Humphcypris chappuisi (Klie, 1935) Martens 1997: OL

Stenocypris chappuisi Klie, 1935

Humphcypris condarensis (Akatova, 1950) Martens 1997: PA

Stenocypris condarensis Akatova, 1950

Humphcypris decipiens (Klie, 1939) Martens 1997: AT

Stenocypris decipiens Klie, 1939

Humphcypris exigua (Rome, 1953) Martens 1997: AT

Stenocypris exigua Rome, 1953

Humphcypris greenwoodi Martens, 1997: AT

Humphcypris leleupi (Harding, 1955) Martens 1997: AT

Stenocypris leleupi Harding, 1955

Humphcypris sewelli (Klie, 1927) Martens \& Savatenalinton 2011: OL

Stenocypris sewelli Klie, 1927 
*Humphcypris subterranea (Hartmann, 1964) Martens 1997: AT, PA

Stenocypris subterranea Hartmann, 1964

Humphcypris thysvillensis (Harding, 1955) Martens 1997: AT

Stenocypris thysvillensis Harding, 1955

Humphcypris tumens (Rome, 1977) Martens 1997: AT

Stenocypris tumens Rome, 1977

Humphreyscandona Karanovic \& Marmonier, 2003

*Humphreyscandona adorea Karanovic \& Marmonier, 2003: AU

Humphreyscandona akaina Karanovic, 2007: AU

Humphreyscandona capillus Karanovic, 2007: AU

Humphreyscandona fovea Karanovic \& Marmonier, 2003: AU

Humphreyscandona imperfecta Karanovic, 2005: AU

Humphreyscandona janeae Karanovic, 2007: AU

Humphreyscandona pilbarae Karanovic \& Marmonier, 2003: AU

Humphreyscandona ventosa Karanovic, 2007: AU

Humphreyscandona waldockae Karanovic \& Marmonier, 2003: AU

Humphreyscandona woutersi Karanovic \& Marmonier, 2003: AU

Hungarocypris Vávra, 1906

Hungarocypris asymmetrica Victor \& Fernando, 1981: AU

Hungarocypris gawemuelleri Vávra, 1906: OL

Hungarocypris levigata Chen, 1991: PA

*Hungarocypris madaraszi (Örley, 1886) Vávra, 1906: PA

Notodromas madaraszi Örley, 1886

Syn.: Cyprois dispar Chyzer, 1858 (non Fischer, 1851) (fide G.W. Müller 1912)

Hungarocypris serrata Chen, 1983: PA

Hungarocypris suranareeae Savatenalinton \& Suttajit, 2016: OL

*Hypselecypris wittei Rome, 1965: AT

Hypselecypris Rome, 1965

Ilyocypris Brady \& Norman, 1889

Syn.: Ilyocyprella Daday, 1900 (fide G.W. Müller 1912)

Syn.: Ilyocyprois Masi, 1906 (fide G.W. Müller 1912)

Ilyocypris aestivalis Fuhrmann, 2008: PA

Ilyocypris alta Sars, 1910: AT

Ilyocypris angulata Sars, 1903: OL, PA

Ilyocypris australiensis Sars, 1889: AT, AU, OL, PA

Ilyocypris botniensis Kovalenko, 1972: PA

Ilyocypris bradyi Sars, 1890: NA, NT, OL, PA

Syn.: Ilyocypris gibba var. repens Vávra, 1891 (fide Klie 1938a)

Syn.: Ilyocyprella repens Daday, 1900 (fide Klie 1938a)

Syn.: Ilyocypris bradyi var. compressa Masi, 1906 (fide Martens \& Savatenalinton 2011)

Syn.: Ilyocypris bradyi var. brachytela Gauthier, 1938 (fide Martens \& Savatenalinton 2011)

Ilyocypris brehmi Schäfer, 1952: PA

Syn.: Ilyocypris turca Ghetti, 1972 (fide Martens 1991)

Ilyocypris decipiens Masi, 1905: NA, PA

Syn.: Ilyocypris iners Kaufmann, 1900 (unused older synonym) (fide Meisch 2000)

Syn.: Ilyocypris iners var. affinis Masi, 1906 (fide Pieri et al. 2015)

Syn.: Ilyocypris almi Sywula, 1968 (fide Meisch 2000)

Syn.: Ilyocypris decipiens f. almi Sywula, 1974 (fide Meisch 2000) 
Ilyocypris dentifera Sars, 1903: OL, PA

Ilyocypris divisa Klie, 1926: PA

Ilyocypris echinata Huang, 1979: PA

Ilyocypris fallax Brehm, 1929: AU

Ilyocypris getica Masi, 1906: PA

*Ilyocypris gibba (Ramdohr, 1808) Brady \& Norman 1889: NA, NT, OL, PA

Cypris gibba Ramdohr, 1808

Syn.: Cypris biplicata Koch, 1838 (fide Meisch 2000)

Ilyocypris hanguk Karanovic \& Lee, 2013: PA

Ilyocypris hartmanni Lerner-Seggev, 1968: PA

Ilyocypris inermis Kaufmann, 1900: PA

Ilyocypris innermongolica Zhai \& Xiao, 2013: PA

Ilyocypris japonica Okubo, 1990: PA

Syn.: Ilyocypris haterumensis Okubo, 1992 (fide Okubo 2004)

Ilyocypris kashmirensis Bhatia, 1968: OL

Ilyocypris lacustris Kaufmann, 1900: PA

Ilyocypris mckenziei Bhatia \& Mannikeri, 1975: OL

Ilyocypris microspinata Huang, 1982: PA

Ilyocypris mongolica Martens, 1991: PA

Ilyocypris monstrifica (Norman, 1862) McKenzie 1970: PA

Cypris monstrifica Norman, 1862

Syn.: Ilyocypris tuberculata Brady, 1868 (fide Meisch 2000)

Syn.: Ilyocypris ambigua Lowndes, 1931 (fide Meisch 2000)

Ilyocypris montana Margalef, 1952: PA

Ilyocypris nagamalaiensis Victor \& Michael, 1975: OL

Ilyocypris neoaspera Huang et al., 1983: PA

Ilyocypris nitida Lerner-Seggev, 1968: PA

Ilyocypris perigundi De Deckker, 1981: AU

Ilyocypris propinqua Sars, 1910: AT

Ilyocypris ramirezi Cusminsky \& Whatley, 1996: NT

Ilyocypris salebrosa Stepanaitys, 1960: NA, OL, PA

Syn.: Ilyocypris shawneetownensis Staplin, 1963 (fide Bates et al. 2002)

Syn.: Ilyocypris steegeri Kempf, 1967 (fide Bates et al. 2002)

Syn.: Pelocypris alatabulbosa Delorme, 1970 (fide Bates et al. 2002)

Ilyocypris salebrosa var. carinata (Kovalenko, 1970) Kovalenko 1976

Ilyocypris carinata Kovalenko, 1970

Ilyocypris salina (Daday, 1910) Martens 1984: AT

Ilyocyprella salina Daday, 1910

Ilyocypris sebeiensis Yang \& Sun, 2004: PA

Ilyocypris subpulchra Yang, 1982: PA

Ilyocypris taprobanensis Neale, 1976: OL

Ilyocypris xizangensis Yang, 1982: PA

Ilyodromus amplicolis De Deckker, 1981: AU

Ilyodromus Sars, 1894

Ilyodromus anisitsi (Daday, 1905) G.W. Müller 1912: NT

Eucypris anisitsi Daday, 1905

Ilyodromus armacutis Shearn et al., 2017: AU

Ilyodromus candonites De Deckker, 1981: AU

Ilyodromus dikrus De Deckker, 1981: AU

Ilyodromus hiatus Shearn et al., 2017: AU 
Ilyodromus intermedius Okubo, 2011: PA

Syn.: Ilyodromus intermedius Okubo, 2004 (nom. nud., no type material designated; fide Smith et al. 2011)

Ilyodromus kerguelensis G.W. Müller, 1906: ANT

Ilyodromus lanaoensis Victor \& Fernando, 1981: OL

Ilyodromus obtusus Sars, 1894: AU

Ilyodromus pectinatus Sharpe, 1908: NA

Ilyodromus sensaddito Shearn et al., 2017: AU

Ilyodromus smaragdinus Sars, 1894: AU, PA

*Ilyodromus stanleyanus (King, 1855) Sars 1894: AU

Candona stanleyana King, 1855

Ilyodromus substriatus Sars, 1894: AU

Ilyodromus varrovillius (King, 1855) Sars 1894: AU

Cypris varrovillia King, 1855

Ilyodromus verreauxii (Baird, 1862) Martens \& Behen 1994: NT

Cypris verreauxii Baird, 1862

Ilyodromus viridulus (Brady, 1886) Sars 1896: AT, AU, PA

Cypris viridula Brady, 1886

Ilyodromus williamsi (McKenzie, 1966) Shearn et al. 2017: AU

Isocypris williamsi McKenzie, 1966

Indiacypris Hartmann, 1964

Indiacypris chalakkudensis George \& Martens, 2004: OL

*Indiacypris dispar Hartmann, 1964: OL

Indiacypris luxata (Brady, 1886) McKenzie 1972: OL

Cypris luxata Brady, 1886

*Indocandona krishnakanti Gupta, 1984: OL

Indocandona Gupta, 1984

Indocandona nagarjuna Karanovic \& Ranga Reddy, 2008: OL

Intrepidocythere Pinto et al., 2008

*Intrepidocythere ibipora Pinto et al., 2008: NT

Isabenula Rossetti et al., 2011

*Isabenula humphreysi Rossetti et al., 2011: OL

Isocypris G.W. Müller, 1908

Syn.: Hyalocypris Brady, 1913 (fide Martens 2001b)

Isocypris africana (Brady, 1913) Sars 1924a: AT

Hyalocypris africana Brady, 1913

Isocypris beauchampi (Paris, 1920) Herbst 1951: NA, NT, PA

Stenocypria beauchampi Paris, 1920

Syn.: Isocypris arnoldi Dubowsky, 1927 (fide Meisch 2000)

Syn.: Stenocypria longicomosa Furtos, 1933 (fide Martens \& Savatenalinton 2011)

Syn.: Isocypris quadrisetosa Rome, 1947 (fide Meisch 2000)

Syn.: Isocypris nocentiniae Fox, 1963 (fide Meisch 2000)

Syn.: Isocypris beauchampi var. cicatricosa Fox, 1963 (fide Meisch 2000)

Syn.: Isocypris foxi Moroni \& McKenzie, 2007 (fide Pieri et al. 2015)

Isocypris laskaridisi Herbst, 1951: AU

Isocypris nivea Sars, 1924: AT

Isocypris perangusta G.W. Müller, 1908: AT

*Isocypris priomena G.W. Müller, 1908: AT, PA 
Kapcypridopsis McKenzie, 1977

Kapcypridopsis asymmetra De Deckker, 1981: AU

* Kapcypridopsis barnardi McKenzie, 1977: AT

Kapcypridopsis megapodus Cusminsky et al., 2005: NT

Kavalacythereis Wouters, 1979

*Kavalacythereis braconensis Wouters, 1979: AT

Kempfcyclocypris Karanovic, 2011

*Kempfcyclocypris australis Karanovic, 2011: AU

Kencandona Karanovic, 2007

* Kencandona harleyi Karanovic, 2007: AU

Kencandona verrucosa Karanovic, 2007: AU

Kennethia De Deckker, 1979

*Kennethia cristata De Deckker, 1979: AU

Kennethia major (Méhes, 1939) De Deckker 1979a: NT, PAC

Notodromas major Méhes, 1939

Kennethia thomseni (Klie, 1935) Martens \& Savatenalinton 2011: NT

Notodromas thomseni Klie, 1935

*Kiwicythere anneari Martens, 1992: AU

Kiwicythere Martens, 1992

Kiwicythere vulgaris (McKenzie \& Swanson, 1981) Martens 1992: AU

Paralimnocythere vulgaris McKenzie \& Swanson, 1981

*Kliella hyaloderma Schäfer, 1945: PA

Kliella Schäfer, 1945

Klieopsis Martens et al., 1991

*Klieopsis horai (Klie, 1927) Martens et al. 1991: AT, OL

Cypridopsis horai Klie, 1927

Syn.: Cypridopsis caerulescens Klie, 1939 (fide Martens et al. 1991)

Koencypris Meisch, 2000

*Koencypris ornata (O.F. Müller, 1776) Meisch 2000: PA

Cypris ornata O.F. Müller, 1776

Korannacythere Martens, 1996

*Korannacythere devriesi Martens, 1996: AT

Korannacythere hamerae Martens, 1996: AT

Korannacythere ugiensis Martens, 1996: AT

Kovalevskiella Klein, 1963

Type species: Kovalevskiella turianensis Klein, 1963: PA (fossil species)

Kovalevskiella bulgarica (Danielopol, 1970) Danielopol 1981: PA

Cordocythere bulgarica Danielopol, 1970

Kovalevskiella cvetkovi (Danielopol, 1969) Danielopol 1971: PA

Cordocythere cvetkovi Danielopol, 1969

Kovalevskiella dani Karanovic, 2003: PA

Kovalevskiella phreaticola (Danielopol, 1965) Danielopol 1971: PA

Cordocythere phreaticola Danielopol, 1965

Kovalevskiella rudjakovi (Danielopol, 1969) Danielopol 1971: PA

Cordocythere rudjakovi Danielopol, 1969 
Laccocythere Hart \& Hart, 1971

*Laccocythere aotearoa Hart \& Hart, 1971: AU

Lacrimacandona Külköylüoğlu et al., 2017

*Lacrimacandona wisei Külköylüoğlu et al., 2017: NA

Lacrimicypris Halse \& McRae, 2004

*Lacrimicypris kumbar Halse \& McRae, 2004: AU

Latinopsis Karanovic \& Datry, 2009

Latinopsis columbienensis (Méhes, 1914) Karanovic \& Datry 2009: NT

Candona columbienensis Méhes, 1914

Latinopsis falklandica (Vávra, 1898) Karanovic \& Datry 2009: NT

Candonopsis falklandica Vávra, 1898

*Latinopsis patagonica Karanovic \& Datry, 2009: NT

Leicacandona Karanovic, 2007

Leicacandona carinata Karanovic, 2007: AU

Leicacandona gyralea Karanovic, 2007: AU

*Leicacandona halsei Karanovic, 2007: AU

Leicacandona jimi Karanovic, 2007: AU

Leicacandona jula Karanovic \& McKay, 2010: AU

Leicacandona lite Karanovic, 2007: AU

Leicacandona makra Karanovic, 2007: AU

Leicacandona mookae Karanovic, 2007: AU

Leicacandona pinkajartinyi Karanovic \& McKay, 2010: AU

Leicacandona quasihalsei Karanovic, 2007: AU

Leicacandona quasimookae Karanovic, 2007: AU

Leicacandona yandagoogeae Karanovic, 2007: AU

Leptocythere Sars, 1925

Type species: Leptocythere pellucida (Baird, 1850) Sars 1925 (marine species)

Leptocythere baltica Klie, 1929: PA

Leptocythere bacuana (Livental, 1938) Mandelstam 1962: PA

Cythere bacuana Livental, 1938

Leptocythere castanea (Sars, 1866) Sars 1925: PA

Cythere castanea Sars, 1866

Leptocythere darbyi Keyser, 1976: NA

Leptocythere devexa Schornikov, 1966: PA

Leptocythere (Leptocythere) devexa Schornikov, 1966

Leptocythere fluviatilis Klie, 1939: PA

Leptocythere gracilloides Schornikov, 1964: PA

Leptocythere histriana Caraion, 1964: PA

Leptocythere (Leptocythere) histriana Caraion, 1964

Leptocythere lacertosa (Hirschmann, 1912) Klie 1929: PA

Cythere lacertosa Hirschmann, 1912

Leptocythere lacustris De Deckker, 1981: AU

Leptocythere lopatici Schornikov, 1964: PA

Leptocythere nitida Schornikov, 1966: PA

Leptocythere (Leptocythere) nitida Schornikov, 1966

Leptocythere ostrovskensis Petkovski \& Keyser, 1992: PA 
Leptocythere pediformis Schornikov, 1966: PA

Leptocythere (Leptocythere) pediformis Schornikov, 1966

Leptocythere polymorpha Schornikov, 1974: PA

Leptocythere pseudoproboscidea Karanovic \& Petkovski, 1999: PA

Leptocythere relicta Schornikov, 1964: PA

Leptocythere reticulata Schornikov, 1966: PA

Leptocythere (Leptocythere) reticulata Schornikov, 1966

Leptocythere triangulata (Löffler, 1959) Griffiths et al. 2001: PA

Cythere triangulata Löffler, 1959

Leucocythere Kaufmann, 1892

Leucocythere algeriensis Martens, 1990: PA

Leucocythere dorsotuberosa Huang, 1984: PA

Leucocythere dorsotuberosa var. postilirata (Pang, 1985)

Syn.: Leucocythere postilirata Pang, 1985 (fide Wrozyna et al. 2009)

Leucocythere helenae Martens, 1991: AT

*Leucocythere mirabilis Kaufmann, 1892: PA

Leucocytherella Huang, 1982

Syn.: Limnocytherellina Pang, 1985 (fide Fürstenberg et al. 2015)

*Leucocytherella sinensis Huang, 1982: PA

Syn.: Leucocytherella trinoda Huang, 1982 (fide Fürstenberg et al. 2015)

Syn.: Leucocytherella glabra You \& Huang, 1982 (fide Fürstenberg et al. 2015)

Syn.: Leucocytherella bispinosa (Pang, 1985) (fide Fürstenberg et al. 2015)

Limnocytherellina bispinosa Pang, 1985

Syn.: Leucocytherella biechinata Huang, 1985 (fide Fürstenberg et al. 2015)

Syn.: Leucocytherella kunlunensis (Pang, 1985) (fide Fürstenberg et al. 2015)

Limnocytherellina kunlunensis Pang, 1985

Syn.: Leucocytherella quadriechinata Yang, 1985 (fide Fürstenberg et al. 2015)

Syn.: Leucocytherella quinquechinata Huang, 1985 (fide Fürstenberg et al. 2015)

Syn.: Leucocytherella subtriechinata Huang, 1985 (fide Fürstenberg et al. 2015)

Syn.: Leucocytherella triechinata Huang, 1985 (fide Fürstenberg et al. 2015)

Syn.: Leucocytherella trinoda Huang-Huang et al., 1985 (fide Fürstenberg et al. 2015)

Syn.: Leucocytherella trispinosa (Pang, 1985) (fide Fürstenberg et al. 2015)

Limnocytherellina trispinosa Pang, 1985

Lichnocythere Hart \& Hart, 1967

Lichnocythere synethes Hart \& Hart, 1967: AU

Lichnocythere tubrabucca Hart \& Hart, 1967: AU

*Lichnocythere victoria Hart \& Hart, 1967: AU

Limanocypris Schornikov, 1961

*Limanocypris lurida Schornikov, 1961: PA

Limnocythere Brady, 1868

Syn.: Limnicythere Brady, 1867 (fide G.W. Müller 1912; misspelling)

Syn.: Acanthopus Vernet, 1878 (partim) (fide G.W. Müller 1912)

Limnocythere aethiopica Klie, 1934: AT

Limnocythere africana Klie, 1939: AT

Limnocythere arthuri Löffler, 1961: NT 
Limnocythere aspera Henry, 1923: AU

Limnicythere aspera Henry, 1923

Limnocythere atacamae Brehm, 1935: NT

Limnocythere borisi Martens, 1990: AT

Limnocythere borisi borisi Martens, 1990

Limnocythere borisi awassaensis Martens, 1990

Limnocythere borisi shalaensis Martens, 1990

Limnocythere bradburyi Forester, 1985: NA, NT

Limnocythere cidreirensis Würdig \& Pinto, 1994: NT

Limnocythere coelebs Klie, 1944: AT

Limnocythere conifera Brehm, 1950: AU

Limnocythere cusminskyae Ramón Mercau et al., 2014: NT

Limnocythere cyphoma Smith \& Janz, 2009: PA

Limnocythere dadayi Martens, 1990: AT

Limnocythere dorsosicula De Deckker, 1981: AU

Limnocythere dubiosa Daday, 1903: PA

Limnocythere elongata Delachaux, 1928: NT

Limnocythere fijiensis Brady, 1890: PAC

Limnocythere floridensis Keyser, 1979: NA

Limnocythere friabilis Benson \& McDonald, 1963: NA

Syn.: Limnocythere chippewaensis Staplin, 1963 (fide Swain 1999)

Limnocythere fude Smith \& Janz, 2009: PA

Limnocythere gibbosa Sywula, 1970: PA

Limnocythere glypta Dobbin, 1941: NA

Limnocythere herricki Staplin, 1963: NA

Limnocythere hungarica Daday, 1900: PA (uncertain species, fide Meisch 2000)

*Limnocythere inopinata (Baird, 1843) Brady 1867: AT, NA, PA

Cythere inopinata Baird, 1843

Syn.: Limnocythere incisa Dahl, 1888 (fide Meisch 2000)

Syn.: Limnocythere balatonica Daday, 1900 (fide Meisch 2000)

Syn.: Limnocythere mongolica Daday, 1901 (fide Meisch 2000)

Syn.: Limnocythere sappaensis Staplin, 1963 (fide Meisch 2000)

Syn.: Limnocythere binoda Huang, 1964 (fide Meisch 2000)

Syn.: Limnocythere inopinata orientalis Ghetti, 1972 (fide Meisch 2000)

Limnocythere iowensis Danforth, 1948: NA

Limnocythere jocquei Martens, 1990: AT

Limnocythere kamiyai Smith \& Janz, 2009: PA

Limnocythere levigatus Smith \& Janz, 2009: PA

Limnocythere liporeticulata Delorme, 1968: NA

Limnocythere marshi Brehm, 1924: NT

Limnocythere michaelseni Daday, 1910: AT

Limnocythere milta De Deckker, 1981: AU

Limnocythere minor Lindroth, 1953: AT

Limnocythere africana minor Lindroth, 1953 (fide Martens 1990a)

Limnocythere mowbrayensis Chapman, 1914: AU

Limnocythere neotropica Klie, 1934: NT

Limnocythere notodonta Vávra, 1906: AT, PAC

Limnocythere opesta Brehm, 1939: NT

Limnocythere ornata Furtos, 1933: NA

Limnocythere oughtoni Tressler, 1957: NA 
MEISCH C. et al., Global checklist of extant non-marine Ostracoda

Limnocythere paranensis Ferguson, 1967: NT

Limnocythere patagonica Cusminsky \& Whatley, 1996: NT

Limnocythere percivali Brehm, 1939: AU

Limnocythere platyforma Delorme, 1971: NA

Limnocythere porphyretica De Deckker, 1981: AU

Limnocythere reticulata Sharpe, 1897: NA

Limnocythere rionegroensis Cusminsky \& Whatley, 1996: NT

Limnocythere robusta Delorme, 1967: NA

Limnocythere royi Hartmann, 1959: NT

Limnocythere scutariense Petkovski, 1961: PA

Limnocythere staplini Gutentag \& Benson, 1962: NA

Limnocythere stationis Vávra, 1891: AT, PA, OL

Limnocythere thomasi Martens, 1990: AT

Limnocythere thomasi thomasi Martens, 1990

Limnocythere thomasi langanoensis Martens, 1990

Limnocythere titicaca Lerner-Seggev, 1973: NT

Limnocythere tudoranceai Martens, 1990: AT

Limnocythere viaticum Allison \& Holden, 1971: PAC

Limnocytherina Negadaev-Nikonov, 1967

Type species: Limnocytherina manjtschensis (Negadaev-Nikonov, 1955) Negadaev-Nikonov 1967 (fossil species)

Limnocythere manjtschensis Negadaev-Nikonov, 1955

Limnocytherina axalapasco Cohuo-Durán et al., 2014: NT

Limnocytherina camera (Delorme, 1967) Delorme 1971: NA

Limnocythere camera Delorme, 1967

Limnocytherina ceriotuberosa (Delorme, 1967) Delorme 1971: NA

Limnocythere ceriotuberosa Delorme, 1967

Limnocytherina illinoisensis (Sharpe, 1897) Delorme 1971: NA

Limnicythere illinoisensis Sharpe, 1897

Limnocytherina itasca (Cole, 1949) Delorme 1971: NA

Limnocythere itasca Cole, 1949

Limnocytherina paraornata Delorme, 1971: NA

Limnocytherina parascutariense Delorme, 1971: NA

Limnocytherina posterolimba (Delorme, 1967) Delorme 1971: NA

Limnocythere posterolimba Delorme, 1967

Limnocytherina pseudoreticulata (Staplin, 1963) Delorme 1971: NA

Limnocythere pseudoreticulata Staplin, 1963

Limnocytherina sanctipatricii (Brady \& Robertson, 1869) Negadaev-Nikonov 1967: NA, PA

Limnicythere sanctipatricii Brady \& Robertson, 1869

Syn.: Acanthopus elongatus Vernet, 1878 (fide Kaufmann 1896)

Syn.: Limnocythere neocomensis Zschokke, 1894 (fide Kaufmann 1896)

Limnocytherina sharpei (Staplin, 1963) Martens \& Savatenalinton 2011: NA

Limnocythere sharpei Staplin, 1963

Limnocytherina varia (Staplin, 1963) Delorme 1971: NA

Limnocythere varia Staplin, 1963

Limnocytherina verrucosa (Hoff, 1942) Delorme 1971: NA

Limnocythere verrucosa Hoff, 1942

*Liocypris grandis Sars, 1924: AT

Liocypris Sars, 1924 
Litocythere Hobbs \& Walton, 1968

*Litocythere lucileae Hobbs \& Walton, 1968: NA

Lordocythere Hobbs \& Hobbs, 1970

*Lordocythere petersi Hobbs \& Hobbs, 1970: NA

\section{Loxoconcha Sars, 1866}

Loxoconcha elliptica Brady, 1868: PA

Syn.: Loxoconcha gauthieri Klie, 1929 (fide Athersuch et al. 1989)

Syn.: Loxoconcha emelwardensis Redeke, 1936 (fide Wagner 1957)

Loxoconcha galilea Lerner-Seggev, 1968: PA

Loxoconcha immodulata Stepanaitys, 1958: PA

*Loxoconcha rhomboidea (Fischer, 1855) Wagner 1957: PA

Cythere rhomboidea Fischer, 1855

Madagascarcypris Martens, 1986

*Madagascarcypris voeltzkowi (G.W. Müller, 1898) Martens 1986: AT

Cypris voeltzkowi G.W. Müller, 1898

Mangalocypria Wouters, 1998

Mangalocypria africana (Hartmann, 1974) Wouters 1998: AT, PAC

Thalassocypria africana Hartmann, 1974

*Mangalocypria appendix Wouters, 1998: PAC

Mangalocypria eleotridis (Harding, 1962) Wouters 1998: AU, PAC

Paracyria eleotridis Harding, 1962

Mangalocypria ryukyuensis Hiruta \& Kakui, 2016: PA

Marmocandona Danielopol et al., 2012

Marmocandona delamarei (Danielopol, 1973) Danielopol et al. 2012: PA

Pseudocandona delamarei Danielopol, 1973

Marmocandona pescei (Karanovic, 2005) Danielopol et al. 2012: PA

Typhlocypris (Typhlocypris) pescei Karanovic, 2005

Marmocandona rouchi (Danielopol, 1973) Danielopol et al. 2012: PA

Pseudocandona rouchi Danielopol, 1973

Marmocandona serfozoi (Gidó, 2010) Danielopol et al. 2012: PA

Pseudocandona serfozoi Gidó, 2010

*Marmocandona zschokkei (Wolf, 1920) Danielopol et al. 2012: PA

Candona zschokkei Wolf, 1920

Marococandona Marmonier et al., 2005

*Marococandona danielopoli Marmonier et al., 2005: AT

Marococandona nicolae Marmonier et al., 2005: AT

Martenscypridopsis Karanovic \& Pesce, 2000

* Martenscypridopsis materia Karanovic \& Pesce, 2000: AT

Martenscypridopsis pygmaea (Sars, 1924) Karanovic \& Pesce 2000: AT

Cypridopsis pygmaea Sars, 1924

Mecynocypria Rome, 1962

Mecynocypria alta Rome, 1962: AT

Mecynocypria complanata (Sars, 1910) Martens 1984: AT

Paracypria complanata Sars, 1910 
Mecynocypria conoidea (Sars, 1910) Martens 1984: AT

Paracypria conoidea Sars, 1910

Mecynocypria curta (Sars, 1910) Martens 1984: AT

Paracypria curta Sars, 1910

Mecynocypria declivis (Sars, 1910) Martens 1984: AT

Paracypria declivis Sars, 1910

Mecynocypria deflexa (Sars, 1910) Rome 1962: AT

Paracypria deflexa Sars, 1910

Mecynocypria emaciata Rome, 1962: AT

Mecynocypria granulata Rome, 1965: AT

Mecynocypria lata Rome, 1962: AT

Mecynocypria limnalis Rome, 1965: AT

*Mecynocypria obtusa (Sars, 1910) Rome 1962: AT

Paracypria obtusa Sars, 1910

Mecynocypria opaca (Sars, 1910) Rome 1962: AT

Paracypria opaca Sars, 1910

Mecynocypria ovata Rome, 1962: AT

Mecynocypria parvula Rome, 1962: AT

Mecynocypria perlonga Rome, 1962: AT

Mecynocypria subangulata (Sars, 1910) Martens 1984: AT

Paracypria subangulata Sars, 1910

Mecynocypria tumidosa Rome, 1962: AT

Megalocypris Sars, 1898

Megalocypris durbani (Baird, 1862) Sars 1924a: AT

Candona durbani Baird, 1862

Megalocypris hispida Sars, 1924: AT

*Megalocypris princeps Sars, 1898: AT

*Meischcandona boitanii Karanovic, 2001: AT

Meischcandona Karanovic, 2001

Meridiescandona Karanovic, 2003

Meridiescandona facies Karanovic, 2003: AU

* Meridiescandona lucerna Karanovic, 2003: AU

Meridiescandona marillanae Karanovic, 2007: AU

Mesocyprideis Wouters \& Martens, 1992

Nom. nov. pro Mesocythere Kiss, 1959 nec Hartmann, 1956

*Mesocyprideis irsacae (Kiss, 1959) Wouters \& Martens 1992: AT

Mesocythere irsacae Kiss, 1959

Mesocyprideis nitida Wouters \& Martens, 2001: AT

Mesocyprideis pila Wouters \& Martens, 1999: AT

Mesocypris Daday, 1910

Mesocypris madagascariensis Danielopol \& Betsch, 1980: AT

Mesocypris pauliani Danielopol \& Betsch, 1980: AT

*Mesocypris pubescens Daday, 1910: AT

Mesocypris terrestris Harding, 1953: AT 
Metacypris Brady \& Robertson, 1870

Syn.: Thaicythere Savatenalinton et al., 2008 (fide Karanovic 2009)

* Metacypris cordata Brady \& Robertson, 1870: PA

Syn.: Metacypris cordata neocomensis Thiébaud, 1906 (fide Meisch 2000)

Metacypris digitiformis Smith \& Hiruta, 2004: PA

Metacypris hebeiensis Huang, 1985: PA

Metacypris lijiangensis Huang, 1982: PA

Metacypris srisumonae (Savatenalinton et al., 2008) Karanovic 2009: OL

Thaicythere srisumonae Savatenalinton et al., 2008

Microdarwinula Danielopol, 1969

Microdarwinula inexpectata Pinto et al., 2005: NT

*Microdarwinula zimmeri (Menzel, 1916) Danielopol 1969: AT, NA, NT, OL, PA

Darwinula zimmeri Menzel, 1916

Microsyssitria Hart et al., 1967

*Microsyssitria indica Hart et al., 1967: OL

Microsyssitria nhlabane Hart \& Clark, 1984: AT

Mixtacandona Klie, 1938

Syn.: Trapezicandona Schornikov, 1969 (fide Namiotko \& Danielopol 2002)

Mixtacandona botosaneanui Danielopol, 1973: PA

Mixtacandona chappuisi (Klie, 1943) Danielopol 1980: PA

Candona chappuisi Klie, 1943

Mixtacandona coineauae Rogulj \& Danielopol, 1993: PA

Mixtacandona cottarellii Danielopol, 1982: PA

Mixtacandona elegans Danielopol \& Cvetkov, 1979: PA

Mixtacandona hvarensis (Danielopol, 1969) Danielopol \& Cvetkov 1979: PA

Candona hvarensis Danielopol, 1969

Mixtacandona idrisi Mazzini \& Rossetti, 2017: PA

Mixtacandona juberthieae Danielopol, 1978: PA

*Mixtacandona laisi Klie, 1938: PA

Syn.: Candona stammeri Klie, 1938 (fide Pieri et al. 2015)

Syn.: Mixtacandona laisi vindobonensis Löffler, 1963 (fide Meisch 2000)

Mixtacandona lattingerae Rogulj \& Danielopol, 1993: PA

Mixtacandona ljovuschkini (Rudjakov, 1963) Danielopol \& Cvetkov 1979: PA

Candona ljovuschkini Rudjakov, 1963

Mixtacandona loeffleri Danielopol, 1973: PA

Mixtacandona peliaca (Schäfer, 1945) Danielopol 1986: PA

Candona peliaca Schäfer, 1945

Mixtacandona pietrosanii Danielopol \& Cvetkov, 1979: PA

Mixtacandona pseudocrenulata (Schäfer, 1945) Danielopol 1980: PA

Candona pseudocrenulata Schäfer, 1945

Mixtacandona riongessa (Bronstein, 1947) Danielopol \& Cvetkov 1979: PA

Cryptocandona riongessa Bronstein, 1947

Mixtacandona spandli Rogulj \& Danielopol, 1993: PA

Mixtacandona tabacarui Danielopol \& Cvetkov, 1979: PA

Mixtacandona talianae Gliozzi \& Mazzini, 1998: PA

Syn.: Mixtacandona italica Karanovic \& Pesce, 2001 (fide Mazzini et al. 2017)

Mixtacandona taurica (Schornikov, 1969) Danielopol 1982a: PA

Candona taurica Schornikov, 1969 
Mixtacandona transleithanica (Löffler, 1960) Danielopol 1980: PA

Candona transleithanica Löffler, 1960

Mnementh Martens, 2007

*Mnementh brennei Martens, 2007: AT

Mungava Harding, 1962

Mungava intermedia Wouters, 1987: PAC

Mungava marthapuriae Keyser, 1976: PAC

*Mungava munda Harding, 1962: PAC

Mungava papuensis Wouters, 1987: PAC

Mungava recta Maddocks, 1992: PAC

Mungava woutersi Maddocks, 2005: PAC

Mungava xariessa Maddocks, 2005: PAC

Mytilocypris McKenzie, 1966

Mytilocypris ambiguosa De Deckker, 1978: AU

Mytilocypris coolcalalaya Halse \& McRae, 2004: AU

Mytilocypris henricae (Chapman, 1966) McKenzie \& Pollard 1966: AU

Eucypris henricae Chapman, 1966

Mytilocypris mytiloides (Brady, 1886) McKenzie 1966b: AU

Cypris mytiloides Brady, 1886

Syn.: Mytilocypris praenuncia Chapman, 1936 (fide Martens \& Savatenalinton 2011)

Syn.: * Mytilocypris tasmanica tasmanica McKenzie, 1966 (fide Martens \& Savatenalinton 2011)

Syn.: Mytilocypris tasmanica chapmani McKenzie, 1978 (fide Halse \& McRae 2004)

Syn.: Mytilocypris minuta De Deckker, 1978 (fide Halse \& McRae 2004)

Mytilocypris splendida (Chapman, 1966) De Deckker 1974: AU

Eucypris splendida Chapman, 1966

Namibcypris Martens, 1992

*Namibcypris costata Martens, 1992: AT

Namiotkocypria Külköylüoğlu, 2018

*Namiotkocypria haysensis Külköylüoğlu, 2018: NA

Nannocandona Ekman, 1914

Nannocandona danielopoli Karanovic et al., 2015: PA

* Nannocandona faba Ekman, 1914: PA

Syn.: Nannocandona faba balcanica Sywula, 1968 (fide Martens \& Savatenalinton 2011)

Nannocandona schornikovi Karanovic et al., 2015: PA

Nannocandona stygia Sywula, 1976: PA

Nannokliella Schäfer, 1945

*Nannokliella dictyoconcha Schäfer, 1945: PA

Nealecypris Savatenalinton \& Martens, 2009

Nealecypris clavigera (G.W. Müller, 1898) Nagler et al. 2014: AT, NT

Cypris clavigera G.W. Müller, 1898

Dolerocypris clavigera (G.W. Müller, 1898) G.W. Müller 1912

Tanycypris clavigera (G.W. Müller, 1898) Rome 1965

*Nealecypris obtusa (Klie, 1933) Savatenalinton \& Martens 2009a: AT

Dolerocypris obtusa Klie, 1933

Tanycypris obtusa (Klie, 1933) McKenzie 1971 
Neglecandona Krstić, 2006

Neglecandona angulata (G.W. Müller, 1900) Medici et al. 2011: PA

Candona angulata G.W. Müller, 1900

Candona (Neglecandona) angulata (G.W. Müller, 1900) Medici et al. 2011

non Candona angulata f. meridionalis Petkovski, $1958=$ Candona meridionalis (Petkovski, 1958)

*Neglecandona lindneri (Petkovski, 1969) Krstić 2006: PA

Candona lindneri Petkovski, 1969

Nom. nov. pro Candona neglecta var. tuberculata Lindner, 1923 nec Candona tuberculata

Daday, 1906

Neglecandona lindneri lindneri (Petkovski, 1969) Krstić 2006

Neglecandona lindneri carpathica (Sywula, 1971) Krstić 2006

Candona (Candona) lindneri carpathica Sywula, 1971

Neglecandona neglecta (Sars, 1887) Krstić 2006: PA

Candona neglecta Sars, 1887

Syn.: Candona scharfi Hahn, 1990 (fide Meisch 2000)

Neocypridella Hartmann \& Puri, 1974

Nom. nov. pro Cypridella Vávra, 1895 nec Koninck, 1841

Neocypridella dartevellei (Brehm, 1939) Martens \& Savatenalinton 2011: AT

Cyprinotus dartevellei Brehm, 1939

Neocypridella devexa (Daday, 1910) Martens 1984: AT

Eucypris devexa Daday, 1910 (sensu Martens 1984)

Neocypridella fossulata (Daday, 1910) Martens 1984: AT

Cypridella fossulata Daday, 1910 (sensu Martens 1984)

Syn.: Cypretta reticulata Lowndes, 1932 (fide Martens 1984)

Syn.: Cyprinotus lowndesi Gauthier, 1939 (fide Martens 1984)

Syn.: Eucypris tumida Klie, 1939 (fide Martens 1984)

Syn.: Eucypris brevis Rome, 1962 (fide Martens 1984)

Syn.: Eucypris palustris Rome, 1962 (fide Martens 1984)

*Neocypridella lemurensis (Vávra, 1895) Martens 1984b: AT

Cypridella lemurensis Vávra, 1895

Neocypridopsis Klie, 1940

Syn.: Cypridopsis (Candonella) Claus, 1891 sensu Vávra 1897 (partim) (fide G.W. Müller 1912)

Syn.: Notiocypridopsis De Deckker, 1981 (fide Martens \& Behen 1994)

Neocypridopsis albida (Sars, 1901) Karanovic \& Datry 2009: NT

Paracypridopsis albida Sars, 1901

Neocypridopsis costata (Daday, 1910) Klie 1940: AT

Oncocypris costata Daday, 1910

Syn.: Zonocypris dadayi Lowndes, 1932 nom. nov. pro Z. costata (Daday, 1910) nec Z. costata

(Vávra, 1897) G.W. Müller, 1898

*Neocypridopsis debilis Klie, 1940: NT

Neocypridopsis frigogena (Graf, 1931) Martens \& Behen 1994: ANT, NT

Cypridopsis frigogena Graf, 1931

Neocypridopsis granulosa (Daday, 1902) Martens \& Behen 1994: NA, NT

Potamocypris granulosa Daday, 1902

Neocypridopsis inaudita (Furtos, 1936) Klie 1940: NT

Cypridopsis inaudita Furtos, 1936

Neocypridopsis mexicana (Furtos, 1938) Danielopol 1986: NT

Cypridopsis mexicana Furtos, 1938 
Neocypridopsis montevidea (Vávra, 1898) Martens \& Behen 1994: NT

Cypridopsis (Candonella) montevidea Vávra, 1898

Neocypridopsis nana (Sars, 1901) Klie 1940: NT

Cypridopsella nana Sars, 1901

Neocypridopsis paradisea (Vávra, 1898) Martens \& Behen 1994: NT

Candonella paradisea Vávra, 1898

Neocypridopsis yucatanensis (Furtos, 1936) Klie 1940: NT

Cypridopsis yucatanensis Furtos, 1936

Neolimnocythere Delachaux, 1928

Neolimnocythere erinacea Delachaux, 1928: NT

*Neolimnocythere hexaceros Delachaux, 1928: NT

Neozonocypris Klie, 1944

*Neozonocypris congensis Klie, 1944: AT

Neozonocypris globosa Rome, 1962: AT

Neozonocypris mirabilis Rome, 1962: AT

Newnhamia King, 1855

Newnhamia dumonti George \& Martens, 2003: OL

*Newnhamia fenestrata King, 1855: AU, OL

Syn.: Newnhamia gulielmi King, 1855 (fide De Deckker 1979a)

Newnhamia fuscata (Brady, 1886) G.W. Müller 1912: AU

Notodromas fuscata Brady, 1886

Newnhamia insolita De Deckker, 1979: AU

Newnhamia patagonica (Vávra, 1898) Vávra 1901: NT

Notodromas patagonica Vávra, 1898

Newnhamia petiola De Deckker, 1979: AU

Ngarawa De Deckker, 1979

* Ngarawa dirga De Deckker, 1979: AU

Notacandona Karanovic \& Marmonier, 2003

Notacandona boultoni Karanovic \& Marmonier, 2003: AU

Notacandona gratia (Karanovic, 2005) Karanovic 2007: AU

Origocandona gratia Karanovic, 2005

*Notacandona modesta Karanovic \& Marmonier, 2003: AU

Notocythere antichthon Hart \& Hart, 1967: AU

Notocythere Hart \& Hart, 1967

Notocythere blundelli Hart \& Hart, 1967: AU

Notocythere erica Hart \& Hart, 1967: AU

Notocythere grampians Hart \& Hart, 1967: AU

Notocythere mirranatwa Hart \& Hart, 1967: AU

Notocythere rieki Hart \& Hart, 1967: AU

Notocythere synomodites Hart \& Hart, 1967: AU

*Notocythere syssitos Hart \& Hart, 1967: AU

Notocythere tasmanica Hart \& Hart, 1967: AU

Notodromas Lilljeborg, 1853

*Notodromas monacha (O.F. Müller, 1776) Lilljeborg 1853: NA, PA

Cypris monacha O.F. Müller, 1776 
Syn.: Cypris bimuricata Koch, 1837 (fide G.W. Müller 1912)

Syn.: Cypris nubilosa Koch, 1837 (fide G.W. Müller 1912)

Notodromas oculata Sars, 1903: OL, PA

Notodromas persica Gurney, 1921: PA

Syn.: Notodromas entzi Daday, 1898 (fide Meisch 2000)

Syn.: Notodromas persica dalmatina Petkovski, 1959 (fide Fox 1962)

Notodromas serrata Deb, 1984: OL

Notodromas sinensis Neale \& Zhao, 1991: OL, PA

Notodromas trulla Smith \& Kamiya, 2014: PA

Okriocythere Hart, 1964

*Okriocythere cheia Hart, 1964: NA

Oncocypris G.W. Müller, 1898

Syn.: Oncocypria Daday, 1908 (fide McKenzie 1982)

Oncocypris bhatiai Battish, 1982: OL

Oncocypris chappuisi Klie, 1939: AT

Oncocypris debundshae Green, 1973: AT

Oncocypris euglypha Rome, 1962: AT

Oncocypris muelleri (Daday, 1910) Lowndes 1932: AT

Oncocypria muelleri Daday, 1910

Syn.: Oncocypris omercooperi Lowndes, 1932 (fide Gauthier 1939)

Syn.: Oncocypris worthingtoni Lowndes, 1936 (fide Gauthier 1939)

Oncocypris rostrata Savatenalinton, 2015: OL

Oncocypris schoutedeni Klie, 1938: AT

*Oncocypris voeltzkowi G.W. Müller, 1898: AT, OL

Syn.: Oncocypris pustulosa Gurney, 1916 (fide Gauthier 1939)

Syn.: Oncocypris brehmii Tressler, 1937 (fide Gauthier 1939)

Origocandona Karanovic, 2005

Origocandona ballardi Karanovic, 2007: AU

Origocandona grommike Karanovic, 2007: AU

*Origocandona inanitas Karanovic, 2005: AU

Ornithocythere Hobbs, 1967

Ornithocythere aetodes Hobbs III, 1970: NA

Ornithocythere gypodes Hobbs, 1969: NA

Ornithocythere popi Hobbs III, 1970: NA

Ornithocythere rhea Hobbs III, 1970: NA

Ornithocythere thomai Hobbs \& McClure, 1983: NA

*Ornithocythere waltonae Hobbs, 1967: NA

*Ovambocythere milani Martens, 1989: AT

Ovambocythere Martens, 1989

Paracandona Hartwig, 1899

*Paracandona euplectella (Robertson, 1889) Hartwig 1999: NA, PA

Candona euplectella Robertson, 1889

Paracypretta Sars, 1924

Paracypretta acanthifera Sars, 1924: AT

*Paracypretta ampullacea Sars, 1924: AT 
Paracypretta aratra (Brady, 1904) McKenzie 1971: AT

Cypris aratra Brady, 1904

Paracypretta minor (G.W. Müller, 1914) Martens et al. 1996: AT

Cypris syngramma var. minor G.W. Müller, 1914

Syn.: Paracypretta rubra Sars, 1924 (fide Martens et al. 1996)

Paracypretta syngramma (G.W. Müller, 1908) McKenzie, 1971: AT

Cypris syngramma G.W. Müller, 1908

Paracypria Sars, 1910

Paracypria adnata Smith \& Kamiya, 2006: PA

Paracypria inopinata (Klie, 1939) Wouters 2001: NT

Dolerocypria inopinata Klie, 1939

Paracypria inujimensis (Okubo, 1980) Wouters 1998: PA

Thalasssocypria inujimensis Okubo, 1980

Paracypria longiseta Hiruta \& Kakui, 2016: PA

Paracypria maryboroughensis Hartmann, 1981: AU

Paracypria minuta McKenzie, 1968: AU

Paracypria plumosa Hiruta \& Kakui, 2016: PA

* Paracypria tenuis (Sars, 1905) Sars 1910: AU

Paracypris tenuis Sars, 1905

Paracypria uberis Maddocks, 2005: PAC

Paracyprideis Klie, 1929

* Paracyprideis fennica (Hirschmann, 1909) Klie 1929: PA

Cytheridea fennica Hirschmann, 1909

Paracythereis Delachaux, 1928

*Paracythereis impudica Delachaux, 1928: NT

Paralimnocythere Carbonnel, 1965

Type species: Paralimnocythere bouleigensis Carbonnel, 1965 (fossil species)

Syn.: Limnocythere (Relictocytherina) Negadaev-Nikonov 1968 (fide Meisch 2000)

Non Paralimnocythere Wang, 1989 (= junior homonym; fide Martens 1992b)

Non Paralimnocythere sensu McKenzie \& Swanson, 1981 (= Kiwicythere Martens, 1992b)

Paralimnocythere alata (Klie, 1939) Martens 1992: PA

Limnocythere alata Klie, 1939

Paralimnocythere compressa (Brady \& Norman, 1889) Diebel \& Pietrzeniuk 1969: PA

Limnicythere compressa Brady \& Norman, 1889

Paralimnocythere diebeli (Petkovski, 1969) Diebel \& Pietrzeniuk 1978: PA

Limnocythere diebeli Petkovski, 1969

Paralimnocythere georgevitschi (Petkovski, 1960) Martens 1992: PA

Limnocythere georgevitschi Petkovski, 1960

Paralimnocythere karamani (Petkovski, 1960) Martens 1992: PA

Limnocythere karamani Petkovski, 1960

Paralimnocythere messanai Martens, 1992: PA

Paralimnocythere ochridensis (Klie, 1934) Martens 1992: PA

Limnocythere ochridense Klie, 1934

Paralimnocythere psammophila (Flössner, 1965) Martens 1992: PA

Limnocythere psammophila Flössner, 1965 
Paralimnocythere relicta (Lilljeborg, 1863) Krstić 1981: PA

Cythere relicta Lilljeborg, 1863

Paralimnocythere slavei (Petkovski, 1969) Martens 1992: PA

Limnocythere slavei Petkovski, 1969

Paralimnocythere umbonata (Klie, 1939) Martens 1992: PA

Limnocythere umbonata Klie, 1939

Paranacypris Higuti et al., 2009

*Paranacypris samambaiensis Higuti et al., 2009: NT

Pelocypris Klie, 1939

Syn.: Pseudoilyocypris Ferguson, 1967 (fide Hartmann \& Puri 1974)

*Pelocypris lenzi Klie, 1939: NT

Pelocypris tuberculata (Ferguson, 1967) Delorme 1970: NA

Pseudoilyocypris tuberculata Ferguson, 1967

Penthesilenula Rossetti \& Martens, 1998

Penthesilenula aotearoa (Rossetti et al., 1998) Rossetti \& Martens 1998: AU, NT

Darwinula aotearoa Rossetti et al., 1998

Penthesilenula araucana (Löffler, 1961) Rossetti \& Martens 1998: NT

Darwinula araucana Löffler, 1961

Penthesilenula brasiliensis (Pinto \& Kotzian, 1961) Rossetti \& Martens 1998: AT, AU, NA, NT, OL, PA, PAC

Darwinula africana brasiliensis Pinto \& Kotzian, 1961

Syn.: Darwinula africana Klie, 1935 (fide Martens \& Savatenalinton 2011)

Syn.: Darwinula dicastrii Löffler, 1966 (fide Rossetti \& Martens 1998)

*Penthesilenula incae (Delachaux, 1928) Rossetti \& Martens 1998: NT

Darwinula incae Delachaux, 1928

Penthesilenula kohanga (Rossetti et al., 1998) Rossetti \& Martens 1998: AU

Darwinula kohanga Rossetti et al., 1998

Penthesilenula malayica (Menzel, 1923) Rossetti \& Martens 1998: AT, OL, PAC

Darwinula malayica Menzel, 1923

Penthesilenula reidae Pinto et al., 2004: NT

Penthesilenula repoa (Chapman, 1963) Rossetti \& Martens 1998: AU Darwinula repoa Chapman, 1963

Penthesilenula setosa (Daday, 1902) Rossetti \& Martens 1998: NT Darwinula setosa Daday, 1902

Penthesilenula sphagna (Barclay, 1968) Rossetti \& Martens 1998: AU Darwinula sphagna Barclay, 1968

Pericythere Hartmann, 1957

Pericythere arcuata Hartmann, 1957: NT

*Pericythere foveata Hartmann, 1957: NT

Pericythere marginata Hartmann, 1959: NT

Perissocytheridea Stephenson, 1938

Syn.: Ilyocythere Klie, 1939 (fide Hartmann \& Puri 1974)

Perissocytheridea bicelliforma Swain, 1955: NA

Perissocytheridea bicelliforma var. propsammia Swain, 1955

Perissocytheridea costata (Hartmann, 1957) Hartmann \& Puri 1974: NT

Ilyocythere costata Hartmann, 1957 
Perissocytheridea cribrosa (Klie, 1933) Keyser 1976: NT

Ilyocythere cribrosa Klie, 1933

Perissocytheridea cytheridellaformis Forester, 1975: NA

Perissocytheridea dentatomarginata (Hartmann, 1957) Hartmann \& Puri1974: NT

Ilyocythere dentatomarginata Hartmann, 1957

Perissocytheridea kroemmelbeini Pinto \& Ornellas, 1970: NT

*Perissocytheridea matsoni (Stephenson, 1935) Stephenson 1938: NT

Potamides matsoni Stephenson, 1935

Perissocytheridea meyerabichi (Hartmann, 1953) Benson \& Kaesler 1963: NT

Ilyocythere meyer-abichi Hartmann, 1953

Perissocytheridea plauta Forester, 1975: NA

Perissocytheridea salvadoriana (Hartmann, 1957) Wouters 1994: NT

Elofsonella salvadoriana Hartmann, 1957

Phreatocandona Danielopol, 1973

*Phreatocandona motasi Danielopol, 1973: PA

Phymocythere Hobbs \& Hart, 1966

Phymocythere lophota Hobbs \& Peters, 1993: NA

*Phymocythere phyma (Hobbs \& Walton, 1962) Hobbs \& Hart 1966: NA

Entocythere phyma Hobbs \& Walton, 1962

Physocypria Vávra, 1897

Syn.: Keysercypria Karanovic, 2011 (partim) syn. nov.

Physocypria aethiopica Klie, 1935: AT

Physocypria affinis Klie, 1933: NT

Keysercypria affinis (Klie, 1933) Karanovic 2011

Physocypria biwaensis (Okubo, 1990) Okubo 2004: PA

Cypria biwaense Okubo, 1990 (spelling error?)

*Physocypria bullata (Vávra, 1897) G.W. Müller 1912: AT, PA

Cypria bullata Vávra, 1897

Physocypria capensis (Sars, 1895) Klie 1933: AT, OL, PA

Cypria capensis Sars, 1895

Syn.: Cypria armata G.W. Müller, 1898 (fide Martens 1984)

Physocypria castanea (Brady, 1904) McKenzie 1971: AT

Cypria castanea Brady, 1904

Physocypria circinata Würdig \& Pinto, 1993: NT

Keysercypria circinata (Würdig \& Pinto, 1993) Karanovic 2011

Physocypria crenulata (Sars, 1903) G.W. Müller 1912: NT, OL, PA

Cypria crenulata Sars, 1903

Physocypria dadayi Martens \& Savatenalinton, 2011: AT

Nom. nov. pro Physocypria denticulata (Daday, 1910) nec Daday, 1905

Cyclocypris denticulata Daday, 1910

Physocypria deformis Klie, 1940: NT

Keysercypria deformis (Klie, 1940) Karanovic 2011

Physocypria denticulata (Daday, 1905) Klie 1935: NA, NT

Cypria denticulata Daday, 1905

Physocypria dentifera (Sharpe, 1918) G.W. Müller 1903: NA

Cypria dentifera Sharpe, 1918

Physocypria devai Arora, 1931: OL

Physocypria dumonti Martens, 1982: AT 
Physocypria exquisita (Furtos, 1936) Tressler 1947: NA

Cypria exquisita Furtos, 1936

Physocypria furfuracea (Brady, 1886) Victor \& Fernando 1979b: OL

Cypris furfuracea Brady, 1886

Physocypria gibbera (Furtos, 1936) Tressler 1947: NA, NT

Cypria gibbera Furtos, 1936

Physocypria granadae Hartmann, 1959: NT

Physocypria inequivalva (Turner, 1893) Tressler 1947: NA

Cypria inequivalva Turner, 1893

Physocypria inflata Furtos, 1933: NA

Physocypria ivanae (Díaz \& Lopretto, 2011) comb. nov.: NT

Keysercypria ivanae Díaz \& Lopretto, 2011

Physocypria kerkyrensis Klie, 1936: PA

Physocypria koenikei (Daday, 1910) Martens 1982: AT (see Taxonomic notes)

Cypria koenikei Daday, 1910

Physocypria kraepelini G.W. Müller, 1903: NA, OL, PA

Syn.: Cypria (Physocypria) fadeewi Dubowsky, 1927 (fide Meisch 2000)

Syn.: Physocypria kliei Schäfer, 1934 (fide Meisch 2000)

Syn.: Cypria inversa Klie, 1941 (fide Meisch 2000)

Syn.: Physocypria perlata Rome, 1943 (fide Meisch 2000)

Physocypria larensis Hartmann, 1964: PA

Physocypria longiseta Klie, 1930: NT

Keysercypria longiseta (Klie, 1930) Karanovic 2011

Physocypria minicapensis Green, 1962: AT

Physocypria minuta Victor \& Michael, 1975: OL

Physocypria nipponica Okubo, 1990: OL, PA

Physocypria pelagica (Brehm, 1932) comb. nov.: NT

Cypria pelagica Brehm, 1932

Syn.: Cypria petenensis Ferguson et al., 1964 syn. nov. nec Klie, 1932

Physocypria posterotuberculata (Furtos, 1935) Tressler 1947: NA

Cypria (Physocypria) posterotuberculata Furtos, 1935

Physocypria pustulosa (Sharpe, 1897) G.W. Müller 1903: NA

Cypria pustulosa Sharpe, 1897

Syn.: Cypria globula Furtos, 1933 (fide Benson \& McDonald 1963)

Physocypria sanctaeannae Margalef, 1961: NT

Keysercypria sanctaeannae (Margalef, 1961) Karanovic 2011

Physocypria schubarti Farkas, 1958: NT

Keysercypria schubarti (Farkas, 1958) Karanovic 2011

Physocypria stricta Rome, 1962: AT

Physocypria tuberata Gurney, 1916: OL

Physocypria xanabanica Furtos, 1936: NT

Cypria (Physocypria) xanabanica Furtos, 1936

Keysercypria xanabanica (Furtos, 1936) Karanovic 2011

Pierrecandona Karanovic, 2007

*Pierrecandona posteriorrecta Karanovic, 2007: AU

Pilbaracandona Karanovic \& Marmonier, 2003

Pilbaracandona colonia Karanovic \& Marmonier, 2003: AU

* Pilbaracandona eberhardi Karanovic \& Marmonier, 2003: AU

Pilbaracandona kosmos Karanovic, 2007: AU 
MEISCH C. et al., Global checklist of extant non-marine Ostracoda

Pilbaracandona rhabdote Karanovic, 2007: AU

Pilbaracandona rosa Karanovic, 2007: AU

Pilbaracandona temporaria Karanovic, 2007: AU

Pioneercandonopsis Karanovic, 2005

*Pioneercandonopsis hancocki Karanovic, 2005: AU

Platycypris Herbst, 1957

*Platycypris baueri Herbst, 1957: AU

Plectocythere Hobbs III, 1965

*Plectocythere crotaphis Hobbs III, 1965: NA

Plectocythere johnsonae Hobbs \& Hart, 1966: NA

Plectocythere kentuckiensis Hobbs \& Peters, 1991: NA

Plectocythere odelli Norden, 1977: NA

Plesiocypridopsis (Rome, 1965) McKenzie 1971

Cypridopsis (Plesiocypridopsis) Rome 1965

Plesiocypridopsis affinis (Klie, 1944) Rome 1965: AT

Cypridopsis affinis Klie, 1944

Plesiocypridopsis alluaudi (Klie, 1935) Martens 1984: AT

Cypridopsis alluaudi Klie, 1935

Plesiocypridopsis angularis (Victor \& Michael, 1975) George \& Martens 2002: OL

Potamocypris angularis Victor \& Michael, 1975

Plesiocypridopsis arsenia (Tressler, 1937) Victor \& Fernando 1982: AU, OL

Cypridopsis arsenia Tressler, 1937

Plesiocypridopsis brachychaeta (Goins, 1972) comb. nov.: NA

Potamocypris brachychaeta Goins, 1972

Plesiocypridopsis chrissiensis (Methuen, 1910) Rome 1965: AT

Cypris chrissiensis Methuen, 1910

Plesiocypridopsis dispar (Hartmann, 1964) Victor \& Fernando 1982: OL

Cypridopsis dispar Hartmann, 1964

Plesiocypridopsis fuelleborni (Daday, 1910) Martens 1984: AT

Potamocypris fuelleborni Daday, 1910

Plesiocypridopsis huaronensis (Delachaux, 1928) Martens \& Behen 1994: NT

Cypridopsis huaronensis Delachaux, 1928

Plesiocypridopsis humilior (Gauthier, 1934) Martens 1984: AT

Cypridopsis humilior Gauthier, 1934

Plesiocypridopsis inaequivalva (Klie, 1933) McKenzie 1971: AT

Cypridopsis inaequivalva Klie, 1933

Plesiocypridopsis insidiosa (Rome, 1965) McKenzie 1971: AT

Cypridopsis insidiosa Rome, 1965

Plesiocypridopsis laevigata (Klie, 1935) Martens 1984: AT

Zonocypris laevigata Klie, 1935

Plesiocypridopsis maduraiensis (Victor \& Michael, 1975) comb. nov.: OL

Cypridopsis maduraiensis Victor \& Michael, 1975

*Plesiocypridopsis newtoni (Brady \& Robertson, 1870) McKenzie 1971: AT, OL, PA

Cypridopsis newtoni Brady \& Robertson, 1870

Syn.: ?Cypridopsis lunata Moniez, 1888 (fide G.W. Müller 1912)

Syn.: Cypridopsis (Candonella) albida Vávra, 1897 (fide Martens \& Savatenalinton 2011)

Syn.: Cypridopsis aldabrae G.W. Müller, 1898 (fide Meisch 2000) 
Syn.: Potamocypris intermedia Daday, 1900 (fide Meisch \& Forró 1998)

Syn.: Cypridopsis pilosa Anichini, 1967 (fide Pieri et al. 2015)

Plesiocypridopsis silvestrii (Daday, 1902) Martens \& Behen 1994: NT

Potamocypris silvestrii Daday, 1902

Plesiocypridopsis thermarum (Tagliasacchi-Masala, 1968) Martens et al. 1991: PA

Cypridopsis thermarum Tagliasacchi-Masala, 1968

Pontoparta Vávra, 1901

Pontoparta eleotridis (Harding, 1962) Maddocks 1992: PAC

Paracypria eleotridis Harding, 1962

Pontoparta hartmanni Keyser, 1975: NA, NT

* Pontoparta rara Vávra, 1901: PAC

Pontoparta salina Harding, 1955: PA

Potamocypris Brady, 1870

Syn.: Cypridopsella Kaufmann, 1900 (fide Meisch 2000)

Syn.: Paracypridopsis Kaufmann, 1900 (fide Meisch 2000)

Syn.: Cyprilla Sars, 1924 (fide Meisch 2000)

Potamocypris altenburgensis Fuhrmann, 2010: PA

Potamocypris alveolata Daday, 1910: AT, NT

Potamocypris arcuata (Sars, 1903) G.W. Müller 1912: PA, NA

Cypridopsella arcuata Sars, 1903

Syn.: Cypris orientalis Baird, 1859 (unused older synonym, fide Meisch 2000)

Syn.: Cypridopsis verrucosa Daday, 1894 (fide Meisch \& Forró 1998)

Syn.: Potamocypris almasyi Daday, 1904 (fide Meisch 2000)

Syn.: Cypridopsis dubia Masi, 1905 (fide Meisch 1985)

Syn.: Potamocypris maculata Alm, 1914 (fide Meisch 2000)

Syn.: Potamocypris longisetosa Bronstein, 1928 (fide Meisch 2000)

Syn.: Potamocypris hyboforma Dobbin, 1941 (fide George \& Martens 2002)

Syn.: Potamocypris almasyi caspica Hartmann, 1964 (fide Meisch 2000)

Syn.: Potamocypris almasyi minor Hartmann, 1964 (fide Meisch 2000)

Syn.: Potamocypris rullieri Anichini, 1968 (fide Meisch 2000)

Syn.: Potamocypris caspica oligogranulata Sywula, 1968 (fide Meisch 2000)

Syn.: Potamocypris caspica macrogranulata Sywula, 1968 (fide Meisch 2000)

Syn.: Potamocypris maculata oligogranulata Sywula, 1974 (fide Meisch 2000)

Syn.: Potamocypris maculata macrogranulata Sywula, 1974 (fide Meisch 2000)

Potamocypris bituminicola Klie, 1940: NT

Potamocypris bowmani Ferguson, 1967: NA

Potamocypris chelazzii Martens, 1982: AT

Potamocypris comosa Furtos, 1933: NA

Potamocypris compressa (Furtos, 1933) Horne et al. 2011: NA

Potamocypris smaragdina var. compressa Furtos, 1933

Potamocypris dadayi (Méhes, 1914) Löffler 1963a: NT

Cypridopsis dadayi Méhes, 1914

Potamocypris deflexa (Sars, 1924) Gauthier 1939: AT

Cyprilla deflexa Sars, 1924

Potamocypris dorsomarginata Rome, 1965: AT

Potamocypris elegantula Furtos, 1933: NA

Potamocypris fallax Fox, 1967: PA

Syn.: Potamocypris wolfi sensu Bronstein 1928, Klie 1938a (fide Meisch 1984)

* Potamocypris fulva (Brady, 1868) Brady 1870: PA

Bairdia fulva Brady, 1868 
MEISCH C. et al., Global checklist of extant non-marine Ostracoda

Potamocypris gibbula (Sars, 1924) Gauthier 1939: AT

Cyprilla gibbula Sars, 1924

Potamocypris humilis (Sars, 1924) Gauthier 1939: AT

Cyprilla humilis Sars, 1924

Potamocypris hummelincki Klie, 1933: NT

Potamocypris illinoisensis Hoff, 1943: NA

Potamocypris insularis Allison \& Holden, 1971: PAC

Potamocypris islagrandensis Hoff, 1943: NA, NT

Potamocypris islagrandensis islagrandensis Hoff, 1943

Potamocypris islagrandensis nicaraguensis Hartmann, 1959

Potamocypris koenikei Daday, 1910: AT

Potamocypris lobata Daday, 1910: AT

Potamocypris mastigophora (Methuen, 1910) Cohen 1986: AT, PA

Cypris mastigophora Methuen, 1910

Syn.: Cyprilla reniformis Brady, 1907 (fide Meisch 1985)

Syn.: Cyprilla producta Sars, 1924 (fide Meisch 1985)

Potamocypris narayanani George \& Martens, 2002: OL

Potamocypris ombrophila Margalef, 1961: NT

Potamocypris opaca Fuhrmann \& Goth, 2011: PA

Potamocypris pallida Alm, 1914: NA, PA

Syn.: Potamocypris thienemanni Klie, 1925 (fide Meisch 2000)

Potamocypris paludum Gauthier, 1939: PA

Nom. nov. pro Cyprilla arcuata Sars, 1924 nec Sars, 1903

Potamocypris philotherma Rome, 1970: PA

Potamocypris problematica Löffler, 1963: NT

Potamocypris reticulata Huang, 1979: PA

Potamocypris saskatchewanensis Ferguson, 1959: NA

Potamocypris schubarti Klie, 1940: NT

Potamocypris similis G.W. Müller, 1912: PA

Nom. nov. pro Potamocypris variegata Kaufmann, 1900 nec Cypridopsis variegata Brady \&

Norman, 1889

Potamocypris smaragdina (Vávra, 1891) Daday 1900: NA, NT, PA

Cypridopsis smaragdina Vávra, 1891

Syn.: Potamocypris dianae Fox, 1965 (fide Meisch 2000)

Potamocypris steueri Klie, 1935: PA

Syn.: Potamocypris tetrataeniata Anichini, 1967 (fide Meisch 1985)

Potamocypris stewarti Daday, 1908: PA

Potamocypris sudzukii Okubo, 1992: OL

Potamocypris tenuilamellata Fuhrmann \& Goth, 2011: PA

Potamocypris unicaudata Schäfer, 1943: NA, NT, PA

Syn.: Potamocypris vanoyei De Vos, 1946 (fide Meisch 2000)

Potamocypris variegata (Brady \& Norman, 1889) Daday 1900: NA, PA

Cypridopsis variegata Brady \& Norman, 1889

Potamocypris villosa (Jurine, 1820) Sars 1890: NT, PA

Monoculus villosa Jurine, 1820

Syn.: Cypridopsis villosa crassipes Masi, 1905 (fide Meisch 2000)

Potamocypris worthingtoni (Lowndes, 1936) Klie 1939a: AT

Cypridopsella worthingtoni Lowndes, 1936

Potamocypris zschokkei (Kaufmann, 1900) G.W. Müller 1912: PA

Paracypridopsis zschokkei Kaufmann, 1900 
Syn.: Potamocypris hambergi hambergi Alm, 1914 (fide Meisch 2000)

Syn.: Potamocypris hambergi rotundata Alm, 1915 (fide Meisch 2000)

Syn.: Potamocypris wolfi Brehm, 1920 (fide Meisch 2000)

Syn.: Potamocypris compacta Klie, 1925 (fide Meisch 2000)

Syn.: Potamocypris tarnogradskyi Bronstein, 1928 (fide Meisch 2000)

Syn.: Potamocypris pyrenaica Margalef, 1947 (fide Meisch 2000)

Syn.: Potamocypris foxi Sywula, 1972 (fide Meisch 2000)

Nom. nov. pro P. wolfi Brehm, 1920 sensu Klie 1938a

Potamocythere Schornikov, 1986

*Potamocythere murgabensis Schornikov, 1986: PA

Prionocypris Brady \& Norman, 1896

Prionocypris gansenensis Huang, 1964: PA

Prionocypris marplesi Chapman, 1963: AU

Prionocypris zenkeri (Chyzer \& Toth, 1858) Lowndes 1931: PA

Cypris zenkeri Chyzer \& Toth, 1858

Syn.: * Prionocypris serrata Norman, 1861 (fide Martens 1989)

Syn.: Cypris bicolor G.W. Müller, 1880 (fide G.W. Müller 1912)

Pseudocandona Kaufmann, 1900

Syn.: Metacandona Bronstein, 1930 (fide Bronstein 1947)

Pseudocandona abei Smith \& Janz, 2008: PA

Pseudocandona academica Mazepova, 1982: PA

Pseudocandona agostinhoi Higuti \& Martens, 2014: NT

Pseudocandona albicans (Brady, 1864) Danielopol 1973: NA, PA

Candona albicans Brady, 1864

Syn.: Candona parallela G.W. Müller, 1900 (fide Meisch 2000)

Syn.: Candona parallela prespica Petkovski, 1959 (fide Meisch 2000)

Syn.: Candona profundicola Löffler, 1960 (fide Meisch 2000)

Syn.: Candona pseudoparallela Löffler, 1961 (fide Meisch 2000)

Syn.: Candona altoalpina Löffler, 1963 (fide Meisch 2000)

Pseudocandona alta Bronstein, 1947: PA

Pseudocandona annae (Méhes, 1914) Broodbakker 1983a: NT

Pseudocandona annae annae (Méhes, 1914) Broodbakker 1983

Candona annae Méhes, 1914

Pseudocandona annae var. septentrionalis (Furtos, 1935) Broodbakker 1983

Candona annae var. septentrionalis Furtos, 1935

Pseudocandona antilliana Broodbakker, 1983: NT

Pseudocandona arcuata (Klie, 1932) comb. nov.: PA

Candona arcuata Klie, 1932

Pseudocandona artuta Mazepova, 1984: PA

Pseudocandona atmeta Smith \& Kamiya, 2015: PA

Pseudocandona bazlikova Mazepova, 1990: PA

Pseudocandona becca Smith \& Kamiya, 2015: PA

Pseudocandona bispinosa (Bronstein, 1930) Bronstein 1947: PA

Metacandona bispinosa Bronstein, 1930

Pseudocandona capitata Mazepova, 1982: PA

Pseudocandona caribbeana Broodbakker, 1983: NT 
MEISCH C. et al., Global checklist of extant non-marine Ostracoda

Pseudocandona carinata (Hartwig, 1901) Fuhrmann 2012: PA

Candona hartwigi carinata Hartwig, 1901

Pseudocandona ceratina Mazepova, 1982: PA

Pseudocandona choi (Karanovic \& Lee, 2012) comb. nov.: PA

Typhlocypris choi Karanovic \& Lee, 2012

Pseudocandona cillisi Higuti \& Martens, 2014: NT

Pseudocandona claudinae Higuti \& Martens, 2014: NT

Pseudocandona complexiva Mazepova, 1985: PA

Pseudocandona compressa (Koch, 1838) Danielopol 1973: NA, PA

Cypris compressa Koch, 1838

Syn.: Candona fallax G.W. Müller, 1900 (fide Meisch 2000)

Syn.: Candona crispata Klie, 1926 (fide Meisch 2000)

Pseudocandona corniculata Mazepova, 1984: PA

Pseudocandona cubensis Broodbakker, 1983: NT

Pseudocandona delormei (Karanovic, 2006) Martens \& Savatenalinton 2011: NA

Typhlocypris delormei Karanovic, 2006

Pseudocandona dorsoconcava (Bronstein, 1947) Danielopol 1980: PA

Candona dorsoconcava Bronstein, 1947

Pseudocandona elliptica (Furtos, 1933) Martens \& Savatenalinton 2011: NA

Candona elliptica Furtos, 1933

Pseudocandona falcula Smith \& Kamiya, 2015: PA

Pseudocandona fluviatilis (Hoff, 1942) Martens \& Savatenalinton 2011: NA

Candona fluviatilis Hoff, 1942

Pseudocandona geratsi Broodbakker, 1983: NT

Pseudocandona glauci Mazepova, 1990: PA

Pseudocandona grumifera Mazepova, 1984: PA

Pseudocandona hartwigi (G.W. Müller, 1900) Martens \& Dumont 1984: PA

Candona hartwigi G.W. Müller, 1900

Syn.: Candona cronebergi Hartwig, 1899 (unused older syn., fide Meisch 2000)

Syn.: Candona absoloni Caraion, 1976 (fide Karanovic 2005)

Pseudocandona herbigrada Mazepova, 1982: PA

Pseudocandona inaequivalvis (Bronstein, 1930) Danielopol 1982b: PA

Pseudocandona inaequivalvis inaequivalvis (Bronstein, 1930)

Candona inaequivalvis inaequivalvis Bronstein, 1930

Pseudocandona inaequivalvis baikalensis (Bronstein, 1930) Danielopol 1982b

Candona inaequivalvis baikalensis Bronstein, 1930

Pseudocandona insculpta (G.W. Müller, 1900) Kaufmann 1900: PA

Candona insculpta G.W. Müller, 1900

Syn.: * Candona pubescens Koch, 1837 (unused older synonym, fide Meisch 2000)

Syn.: Pseudocandona vasconica (Margalef, 1946) comb. nov., syn. nov.

Syn.: Candona vasconica (Margalef, 1946) Margalef 1953 syn. nov.

Syn.: Candona neglecta var. vasconica Margalef, 1946 syn. nov.

Pseudocandona jeanneli (Klie, 1931) Danielopol 1986: NA

Candona jeanneli Klie, 1931

Pseudocandona lobipes (Hartwig, 1900) Wouters 1989: PA

Candona lobipes Hartwig, 1900

Syn.: Candona brevis G.W. Müller, 1900 (fide Meisch 2000)

Pseudocandona lukini Mazepova, 1985: PA

Pseudocandona malomorica Mazepova, 1982: PA 
Pseudocandona marchica (Hartwig, 1899) Carbonnel 1969: PA

Candona marchica Hartwig, 1899

Syn.: Candona rostrata sensu G.W. Müller 1900 (fide Meisch 2000)

Pseudocandona marengoensis (Klie, 1931) Danielopol 1973: NA, OL

Candona marengoensis Klie, 1931

Pseudocandona mira (Sywula, 1976) Danielopol 1980: PA

Candona (Typhlocypris) mira Sywula, 1976

Pseudocandona morimotoi (McKenzie, 1972) Danielopol 1986: PA

Candona morimotoi McKenzie, 1972

Pseudocandona olchonica Mazepova, 1990: PA

Pseudocandona onduotoryensis Mazepova, 1984: PA

Pseudocandona parvispinosa Bronstein, 1947: PA

Pseudocandona parvula (Sars, 1926) Martens \& Savatenalinton 2011: NA, NT

Candona parvula Sars, 1926

Syn.: Candona exilis Furtos, 1933 (fide Karanovic 2007)

Pseudocandona pedropalensis Méhes, 1914 comb. nov.: NT

Candona pedropalensis Méhes, 1914

Pseudocandona pratensis (Hartwig, 1901) Danielopol 1980: PA

Candona pratensis Hartwig, 1901

Syn.: Candona compressa sensu Kaufmann 1900 (fide Meisch 2000)

Syn.: Candona pubescens sensu G.W. Müller 1900 (fide Meisch 2000)

Pseudocandona pseudosetosa Mazepova, 1990: PA

Pseudocandona pumilis Würdig \& Pinto, 1999: NT

Pseudocandona punctata (Furtos, 1933) Swain 1995: NA

Candona punctata Furtos, 1933

Pseudocandona regisnikolai Karanovic \& Petkovski, 1999: PA

Pseudocandona renalis Mazepova, 1982: PA

Pseudocandona renoensis (Gutentag \& Benson, 1962) Martens \& Savatenalinton 2011: NA, PA

Candona renoensis Gutentag \& Benson, 1962

Pseudocandona rostrata (Brady \& Norman, 1889) Carbonnel 1969: NA, PA

Candona rostrata Brady \& Norman, 1889

Pseudocandona sarsi (Hartwig, 1899) Meisch et al. 1990: PA

Candona sarsi Hartwig, 1899

Syn.: Candona dentata G.W. Müller, 1900 (fide Meisch 2000)

Pseudocandona saxatilis Bronstein, 1947: PA

Pseudocandona schmorditzi Fuhrmann \& Goth, 2011: PA

Pseudocandona scita Mazepova, 1982: PA

Pseudocandona semicognita (Schäfer, 1934) Griffiths 1995: PA

Candona semicognita Schäfer, 1934

Pseudocandona setosa Bronstein, 1947: PA

Pseudocandona setosa setosa Bronstein, 1947

Pseudocandona setosa abyssalis Mazepova, 1990

Pseudocandona slavei Pekovski, 1969: PA

Pseudocandona stagnalis (Sars, 1890) Meisch \& Broodbakker 1993: NA, PA

Candona stagnalis Sars, 1890

Syn.: Candona zenkeri Sars, 1890 (fide Klie 1938a)

Syn.: Candona ambigua Scott, 1891 (fide Klie 1938a)

Syn.: Candona rara G.W. Müller, 1900 (fide Klie 1938a)

Syn.: Candona quadrata Alm, 1914 (fide Klie 1938a)

Syn.: Candona stagnalis var. longisetosa Furtos, 1933 (fide Delorme 1970) 
Pseudocandona sucki (Hartwig, 1901) Danielopol 1980: PA

Candona sucki Hartwig, 1901

Syn.: Candona compressa sensu Sars 1925 (fide Sywula 1974)

Pseudocandona tenuirostris Hiruta \& Mawatari, 2013: PA

Pseudocandona triquetroides (Sywula, 1974) Meisch 1996: PA

Candona triquetroides Sywula, 1974

Pseudocandona tuberculata Bronstein, 1947: PA

Pseudocandona tuberculata tuberculata Bronstein, 1947

Pseudocandona tuberculata distorta Mazepova, 1990

Pseudocandona tuberculata huluguneica Mazepova, 1990

Pseudocandona valosa Mazepova, 1982: PA

Pseudocandona valosiformis Mazepova, 1990: PA

Pseudocandona werestschagini (Bronstein, 1930) Bronstein 1947: PA Metacandona werestschagini Bronstein, 1930

Pseudocypretta Klie, 1932

*Pseudocypretta maculata Klie, 1932: OL

Pseudocypridopsis Karanovic, 1999

* Pseudocypridopsis clathrata (Klie, 1937) Karanovic 1999: PA

Cypridopsis clathrata Klie, 1937

Pseudocypridopsis hartmanni Petkovski et al., 2009: PA

Pseudocypridopsis petkovskii Karanovic, 2000: PA

Pseudocypridopsis sywulai Petkovski et al., 2009: PA

Pseudocypris Daday, 1910

Pseudocypris acuta (G.W. Müller, 1914) McKenzie 1971: AT

Cypris acuta G.W. Müller, 1914

Syn.: Pseudocypris testudo Sars, 1924 (fide Martens 1990b)

* Pseudocypris bouvieri Daday, 1910: AT

Pseudocypris circularis Sars, 1924: AT

Pseudocypris expansa Sars, 1924: AT

Pseudocypris gibbera Sars, 1924: AT

Pseudocypris patialaensis Battish, 1977: OL

Pseudocypris spinosa (Methuen, 1910) McKenzie 1971: AT

Cypris spinosa Methuen, 1910

Pseudocypris triquetra Sars, 1924: AT

Pseudolimnocythere Klie, 1938

Pseudolimnocythere hartmanni Danielopol, 1979: PA

*Pseudolimnocythere hypogaea Klie, 1938: PA

Pseudostrandesia Savatenalinton \& Martens, 2009

Pseudostrandesia calapanensis (Tressler, 1937) Savatenalinton \& Martens 2009a: OL, PA

Strandesia calapanensis Tressler, 1937

Syn.: Strandesia uenoi Klie, 1938 (fide Savatenalinton \& Martens 2010)

Pseudostrandesia gaetani Savatenalinton \& Martens, 2010: OL

Pseudostrandesia mamarilorum (Victor \& Fernando, 1981) Savatenalinton \& Martens 2009a: OL

Pseudostrandesia mamarilorum mamarilorum (Victor \& Fernando, 1981) Savatenalinton \& Martens 2009a Strandesia mamarilorum mamarilorum Victor \& Fernando, 1981

Pseudostrandesia mamarilorum sumatrana (Victor \& Fernando, 1981) Savatenalinton \& Martens 2009a Strandesia mamarilorum sumatrana Victor \& Fernando, 1981 
Pseudostrandesia ovata Savatenalinton \& Martens, 2010: OL

Pseudostrandesia phetchabunensis Savatenalinton \& Martens, 2010: OL

* Pseudostrandesia striatoreticulata (Klie, 1932) Savatenalinton \& Martens 2009a: OL

Strandesia striatoreticulata Klie, 1932

Pseudostrandesia thailandensis Savatenalinton \& Martens, 2010: OL

Psittocythere Hobbs \& Walton, 1975

*Psittocythere psitta Hobbs \& Walton, 1975: NA

Psychrodromus Danielopol \& McKenzie, 1977

Psychrodromus betharrami Danielopol \& Baltanás, 1993: PA

Psychrodromus fontinalis (Wolf, 1920) Danielopol \& McKenzie 1977: PA Ilyodromus fontinalis Wolf, 1920

*Psychrodromus olivaceus (Brady \& Norman, 1889) Danielopol \& McKenzie 1977: PA Erpetocypris olivacea Brady \& Norman, 1889

Psychrodromus peristericus (Petkovski, 1959) Danielopol \& McKenzie 1977: PA Ilyodromus peristericus Petkovski, 1959

Psychrodromus robertsoni (Brady \& Norman, 1889) Danielopol \& McKenzie 1977: PA Erpetocypris robertsoni Brady \& Norman, 1889

Psychrodromus tunisicus Zaibi et al., 2013: PA

Psychrodromus turcicus (Hartmann, 1964) Danielopol \& McKenzie 1977: PA Ilyodromus turcicus Hartmann, 1964

Ramotha Martens, 1992

Ramotha capensis (G.W. Müller, 1908) Martens 1992: AT Cypris capensis G.W. Müller, 1908

Ramotha corpulenta (Sars, 1895) Martens 1992: AT Cypris corpulenta Sars, 1895

Ramotha crassa (Klie, 1939) Martens 1992: AT

Strandesia crassa Klie, 1939

Syn.: Cypris dromedarius Fischer, 1851 (fide G.W. Müller 1912)

Syn.: Eucypris bouilloni Kiss, 1959 (fide Martens 1992)

Ramotha curtisae Martens, 1992: AT

* Ramotha hirta (Sars, 1924) Martens 1992: AT

Eucypris hirta Sars, 1924

Syn.: Cypris corpulenta sensu G.W. Müller, 1908 (fide Martens 1992)

Ramotha kenyensis (Lindroth, 1953) Martens 1992: AT

Eucypris kenyensis Lindroth, 1953

Ramotha montana (Lindroth, 1953) Martens 1992: AT

Eucypris montana Lindroth, 1953

Syn.: Eucypris hirta sensu Klie 1944 (fide Martens 1992)

Ramotha producta (Sars, 1924) Martens 1992: AT

Eucypris producta Sars, 1924

Ramotha purcelli (Sars, 1924) Martens 1992: AT

Eucypris purcelli Sars, 1924

Ramotha trichota (G.W. Müller, 1908) Martens 1992: AT

Cypris trichota G.W. Müller, 1908

Ramotha trigona (Sars, 1895) Martens 1992: AT

Cypris trigona Sars, 1895 
Renaudcypris McKenzie, 1980

*Renaudcypris gorongae McKenzie, 1980: AU

Renaudcypris luzonensis Wouters, 1986: OL

Renaudcypris natans Hartmann, 1984: PAC

Renaudcypris wolff (Harding, 1962) McKenzie 1980: PAC

Pontoparta wolffi Harding, 1962

Repandocypris Halse \& McRae, 2004

* Repandocypris austinensis Halse \& McRae, 2004: AU

Repandocypris gleneagles Halse \& McRae, 2004: AU

Reticypris clava De Deckker, 1981: AU

Reticypris McKenzie, 1978

Reticypris dedeckkeri McKenzie, 1978: AU

*Reticypris herbsti McKenzie, 1978: AU

Reticypris kurdimurka De Deckker, 1981: AU

Reticypris pinguis De Deckker, 1981: AU

Reticypris walbu De Deckker, 1979: AU

Rhadinocythere Hart, 1962

*Rhadinocythere serrata (Hoff, 1944) Hart 1962: NA

Entocythere serrata Hoff, 1944

Riekocythere Hart \& Hart, 1967

*Riekocythere cherax Hart \& Hart, 1967: AU

Riekocythere xenika Hart \& Hart, 1967: AU

Riocypris Klie, 1935

Riocypris hinzeae Karanovic, 2008: AU (see Taxonomic notes)

*Riocypris uruguayensis Klie, 1935: NT

Romecytheridea Wouters, 1988

* Romecytheridea ampla Wouters, 1988: AT

Romecytheridea bacata Wouters \& Martens, 2007: AT

Romecytheridea belone Wouters \& Martens, 2001: AT

Romecytheridea concurrens Wouters \& Martens, 2008: AT

Romecytheridea longior Wouters \& Martens, 1999: AT

Romecytheridea plegma Wouters \& Martens, 2001: AT

Romecytheridea tenuisculpta (Rome, 1962) Wouters 1988: AT

Haplocytheridea tenuisculpta Rome, 1962

Rudjakoviella Triebel, 1973

Nom. nov. pro Xenocypris Triebel, 1962 nec Günther, 1868 (genus of fish) (fide Malz 1973)

*Rudjakoviella prolongata (Triebel, 1962) Triebel 1973: NT

Xenocypris prolongata Triebel, 1962

Syn.: Xenocypris procera Triebel, 1962 (nom. nud.) (fide Kempf 1980a)

Rugosuscandona Külköylüoğlu et al., 2017

*Rugosuscandona scharfi Külköylüoğlu et al., 2017: NA

Sagittocythere Hart, 1962

*Sagittocythere barri (Hart \& Hobbs, 1961) Hart 1962: NA

Entocythere barri Hart \& Hobbs, 1961

Sagittocythere stygia Hart \& Hart, 1966: NA 
Sanyuania Zhao \& Han, 1980

Type species: Sanyuania psaronius Zhao \& Han, 1980 (marine fossil species)

Sanyuania segersi Savatenalinton \& Martens, 2009: OL

Sarscypridopsis McKenzie, 1977

*Sarscypridopsis aculeata (Costa, 1847) McKenzie 1977: AT, AU, NA, NT, PA

Cypris aculeata Costa, 1847

Syn.: Cypris aculeata Lilljeborg, 1853 (fide Alm 1915)

Syn.: Potamocypris gregaria Sars, 1895 (fide Sywula 1966)

Syn.: Cypridopsella granulata Sars, 1903 (fide Sywula 1966)

Syn.: Cypridopsis triquetra G.W. Müller, 1908 (fide Martens 1984)

Syn.: Cypridopsis spinifera Sars, 1924 (fide Martens et al. 1996)

Syn.: Cypridopsis obstinata Barclay, 1968 (fide Meisch 2000)

Sarscypridopsis brevis (Sars, 1924) Martens 1984: AT

Cypridopsis brevis Sars, 1924

Sarscypridopsis clavata (Sars, 1924) Martens 1984: AT

Cypridopsis clavata Sars, 1924

Sarscypridopsis echinata (G.W. Müller, 1908) McKenzie 1977: AT

Cypridopsis echinata G.W. Müller, 1908

Sarscypridopsis elizabethae (Sars, 1924) Martens 1984: AT

Cypridopsis elizabethae Sars, 1924

Sarscypridopsis glabrata (Sars, 1924) Martens 1984: AT

Cypridopsis glabrata Sars, 1924

Sarscypridopsis hirsuta (Sars, 1924) Martens 1984: AT

Cypridopsis hirsuta Sars, 1924

Sarscypridopsis katesae (Hartmann, 1957) Martens 1984: AT

Cypridopsis katesae Hartmann, 1957

Sarscypridopsis lanzarotensis (Mallwitz, 1984) Meisch \& Broodbakker 1990: PA

Cypridopsis lanzarotensis Mallwitz, 1984

Sarscypridopsis ochracea (Sars, 1924) McKenzie 1977: AT, AU, OL

Cypridopsis ochracea Sars, 1924

Sarscypridopsis punctata (Sars, 1924) Martens 1984: AT

Cypridopsis punctata Sars, 1924

Sarscypridopsis pyramidata (Sars, 1924) Martens 1984: AT

Cypridopsis pyramidata Sars, 1924

Sarscypridopsis reniformis (Sars, 1924) Martens 1984: AT

Cypridopsis reniformis Sars, 1924

Sarscypridopsis sarsi (Klie, 1935) Martens 1984: AT

Nom. nov. pro Cypridopsis tumidula Sars, 1924 nec Sars, 1910

Cypridopsis sarsi Klie, 1935

Sarscypridopsis striolata (Sars, 1924) McKenzie 1977: AT

Cypridopsis striolata Sars, 1924

Sarscypridopsis tonsa (Sars, 1924) Martens 1984: AT

Cypridopsis tonsa Sars, 1924

Sarscypridopsis trigonella (Sars, 1924) Martens 1984: AT

Cypridopsis trigonella Sars, 1924

Sarsicytheridea Athersuch, 1982

Type species: Cythere bradii Norman, 1865 (marine species)

Syn.: Cytheridea sensu Sars 1925 (fide Athersuch et al. 1989) 
Sarsicytheridea punctillata (Brady, 1865) Athersuch 1982: PA

Cytheridea punctillata Brady, 1865

Syn.: Cyprideis proxima Sars, 1866 (fide Athersuch et al. 1989)

*Saurocythere rhipis Hobbs III, 1969: NA

Saurocythere Hobbs III, 1969

Schellencandona Meisch, 1996

Schellencandona belgica (Klie, 1937) Meisch 1996: PA

Candona belgica Klie, 1937

Schellencandona insueta (Klie, 1938) Meisch 1996: PA

Candona insueta Klie, 1938

*Schellencandona schellenbergi (Klie, 1934) Meisch 1996: PA

Candona schellenbergi Klie, 1934

Schellencandona simililampadis (Danielopol, 1978) Meisch 1996: PA

Pseudocandona simililampadis Danielopol, 1978

Schellencandona tea Karanovic \& Lee, 2012: PA

Schellencandona triquetra (Klie, 1936) Meisch 1996: PA

Candona triquetra Klie, 1936

Schellencandona yakushimaensis Smith \& Kamiya, 2006: PA

Schornikovdona Külköylüoğlu et al., 2017

*Schornikovdona bellensis Külköylüoğlu et al., 2017: NA

Sclerocypris Sars, 1924

Syn.: Bharatcypris Battish, 1978 (fide Martens \& Coomans 1990)

Sclerocypris bhatiai Jain, 1979: OL

Sclerocypris bicornis (G.W. Müller, 1900) Lindroth 1953: AT

Cypris bicornis G.W. Müller, 1900

Syn.: Eucypris elongata Spandl, 1924 (fide Martens \& Coomans 1990)

* Sclerocypris clavularis Sars, 1924: AT

Syn.: Eucypris capensis Daday, 1910 (fide Martens \& Coomans 1990)

Sclerocypris coomansi Martens, 1986: AT

Sclerocypris dayae Martens, 1988: AT

Sclerocypris dedeckkeri Martens, 1988: AT

Sclerocypris demoori Martens, 1991: AT

Sclerocypris devexa (Daday, 1910) Martens 1986: AT

Eucypris devexa Daday, 1910

Sclerocypris dharwadensis Mannikeri \& Vaidya, 1990: OL

Sclerocypris dumonti Martens, 1988: AT

Sclerocypris exserta Sars, 1924: AT

Sclerocypris exserta exserta Sars, 1924

Sclerocypris exserta makarikarensis Martens, 1988

Sclerocypris flabella (Vávra, 1897) Klie 1939: AT

Cypris flabella Vávra, 1897

Sclerocypris jaini Bhatia \& Mannikeri, 1977: OL

Sclerocypris jenkinae Klie, 1933: AT

Sclerocypris longisetosa Martens, 1988: AT

Sclerocypris major Sars, 1924: AT

Sclerocypris methueni (Kempf, 2015): AT

Nom. nov. pro Cypris tuberculata Methuen, 1910 nec Cypris tuberculata Sowerby, 1836 
Sclerocypris tuberculata (Methuen, 1910) Klie 1939

Syn.: Sclerocypris sarsi Martens, 1984 (nom. nov.) (fide Kempf 2015)

Cypris methueni Kempf, 2015 (nom. nov.)

Syn.: Afrocypris biconica Klie, 1933 (fide Martens 1986)

Sclerocypris multiformis (Kiss, 1960) Martens 1984: AT

Candonocypris multiformis Kiss, 1960

Syn.: Eucypris serratamarginata Kiss, 1960 (fide Martens 1986)

Sclerocypris pardii Martens, 1987: AT

Sclerocypris rajasthaniensis Deb, 1973: OL

Syn.: Bharatcypris dentata (Victor \& Michael, 1975) Victor \& Fernando 1981 (fide Martens \&

Savatenalinton 2011)

Syn.: Bharatcypris mackenziei Battish, 1978 (fide Martens 1986)

Syn.: Bharatcypris indica Deb, 1983 (fide Martens 1986)

Sclerocypris rothschildi (Daday, 1910) Martens 1986: AT

Eucypris rothschildi Daday, 1910

Sclerocypris tuberculata (Sars, 1924) McKenzie 1971: AT

Megalocypris tuberculata Sars, 1924

Syn.: Sclerocypris sarsi Martens, 1986 (nom. nov.)

Sclerocypris venusta (Vávra, 1897) Sars 1924b: AT

Cypris venusta Vávra, 1897

Sclerocypris virungensis Martens, 1988: AT

Sclerocypris woutersi Martens, 1988: AT

Sclerocypris zelaznyi Martens, 1988: AT

Sclerocypris zelaznyi zelaznyi Martens, 1988

Sclerocypris zelaznyi etoshensis Martens, 1988

Scottia audax Chapman, 1961: AU

Scottia Brady \& Norman, 1889

Scottia birigida Smith et al., 2002: PA

Scottia insularis Chapman, 1963: AU

*Scottia pseudobrowniana Kempf, 1971: NA, PA

Semicytherura Wagner, 1957

Type species: Semicytherura nigrescens (Baird, 1838) Wagner 1957 (marine species)

Semicytherura gerlachi (Hartmann, 1957) Wouters 1994: NT

Cytherura gerlachi Hartmann, 1957

Siamopsis Savatenalinton, 2017

Siamopsis conspecta Savatenalinton, 2017: OL

Siamopsis khoratensis Savatenalinton, 2017: OL

Siamopsis planitia Savatenalinton, 2017: OL

*Siamopsis renateae Savatenalinton, 2017: OL

Siamopsis sutttajiti Savatenalinton, 2017: OL

Somalicypris Martens, 1997

*Somalicypris uegitia (Masi, 1925) Martens, 1997: AT

Acocypris uegitia Masi, 1925

Sphaeromicola cebennica Remy, 1948: PA

Sphaeromicola Paris, 1916

Sphaeromicola cebennica cebennica Remy, 1948 
Sphaeromicola cebennica juberthiei Danielopol, 1977

Sphaeromicola hamigera (Remy, 1946) Remy 1948: PA

Sphaeromicola stammeri var. hamiger Remy, 1946

Sphaeromicola sphaeromidicola Hubault, 1938: PA

Sphaeromicola stammeri Klie, 1930: PA

*Sphaeromicola topsenti Paris, 1916: PA

Spirocypris Sharpe, 1903

Spirocypris horrida (Sars, 1926) McKenzie 1982: NA

Cypricercus horridus Sars, 1926

*Spirocypris passaica Sharpe, 1903: NA

Spirocypris tuberculata Sharpe, 1908: NA

Stenocypria G.W. Müller, 1901

*Stenocypria fischeri (Lilljeborg, 1883) G.W. Müller 1901: PA

Cypris fischeri Lilljeborg, 1883

Stenocypris acocyproides Klie, 1933: AT

Stenocypris Sars, 1889

Stenocypris acuta (Vávra, 1895) G.W. Müller 1912: AT

Cypris acuta Vávra, 1895

Stenocypris akatovae Semenova, 1996: PA

Stenocypris archoplites Ferguson, 1964: NA

Stenocypris bimucronata Vávra, 1906: OL

Stenocypris bronsteini Semenova, 2006: PA

Stenocypris caesia Klie, 1935: AT

Stenocypris curvirami Lowndes, 1932: AT

Stenocypris damasi Kiss, 1959: AT

Stenocypris decorata Daday, 1910: AT

Stenocypris derupta Vávra, 1906: AT, OL, PA

Stenocypris distincta Victor \& Fernando, 1978: OL

Stenocypris dybowskii Grochmalicki, 1913: AT

Stenocypris elongata Daday, 1910: AT

Stenocypris exsiccata (Vávra, 1897) G.W. Müller 1912: AT

Cypris exsiccata Vávra, 1897

Stenocypris fontinalis (Vávra, 1895) G.W. Müller 1912: AT, AU, NT, PA

Cypris fontinalis Vávra, 1895

Stenocypris hirutai Smith \& Kamiya, 2006: PA

Stenocypris hislopi Ferguson, 1969: OL, PA

Stenocypris ilyophila Klie, 1932: OL

Stenocypris intermedia Klie, 1932: NA, OL, PA

Syn.: Stenocypris bolieki Ferguson, 1962 (fide Martens 2001a)

Syn.: Stenocypris macedonica Petkovski \& Meisch, 1996 (fide Martens 2001a)

Syn.: Stenocypris malcolmsoni lata Ghetti, 1972 (fide Petkovski \& Meisch 1996)

Stenocypris jabalpurensis Harshey \& Patil, 1988: OL

Stenocypris karakalpakii Semenova, 2006: PA

Stenocypris major (Baird, 1859) Daday 1898: AT, NA, NT, OL, PA, PAC

Cypris major Baird, 1859

Stenocypris major major (Baird, 1859) Daday, 1898

Syn.: Stenocypris cylindrica major (Baird, 1859) (fide Martens \& Behen 1994)

Syn.: Cypris chittyensis Baird, 1862 (fide Martens \& Behen 1994) 
Syn.: *Stenocypris malcolmsoni Brady, 1886 (fide Martens \& Behen 1994)

Syn.: Stenocypris major okuboi Petkovski \& Meisch, 1996 (fide Martens 2001a)

Stenocypris major sketi Petkovski \& Meisch, 1996

Stenocypris malayica Victor \& Fernando, 1981: NT, OL, PA, PAC

Stenocypris marginata Daday, 1910: AT, OL, PAC

Stenocypris maxillaris Kiss, 1960: AT

Stenocypris orientalis Victor \& Fernando, 1981: OL

Stenocypris pancratovae Akatova, 1950: PA

Stenocypris pseudoelongata Martens, 1984: AT

Nom. nov. pro Stenocypris elongata Kiss, 1960 nec Daday, 1910

Stenocypris quadridentata Rome, 1962: AT

Stenocypris simulans Rome, 1965: AT, OL

Stenocypris sohni Deb, 1983: OL

Stenocypris stagnalis Daday, 1910: AT

Stenocypris trapezoides Klie, 1932: OL

Stenocypris tsukagoshii Smith \& Kamiya, 2006: PA

Stenocypris ussangiana Grochmalicki, 1913: AT

Stenocypris viridis Okubo, 1990: PA

Strandesia Stuhlmann, 1888

Syn.: Acanthocypris Claus, 1892 (fide Martens \& Behen 1994)

Syn.: Neocypris Sars, 1901 (fide Martens \& Behen 1994)

Syn.: ?Sataracypris Deb, 1983 syn. nov.

Strandesia ambigua (Ghetti, 1972) Rasouli et al. 2016: PA

Eucypris ambigua Ghetti, 1972

Strandesia asymmetros Rome, 1962: AT

Strandesia bicornuta Hartmann, 1964: OL

Strandesia bicuspis (Claus, 1892) G.W. Müller 1912: NA, NT, PA

Acanthocypris bicuspis Claus, 1892

Syn.: Neocypris gladiator Sars, 1901 (fide Martens \& Behen 1994)

Syn.: Strandesia denticulata Tressler, 1950 (juveniles, fide Martens et al. 1998)

Strandesia bicuspis var. mucronata (Claus, 1892) G.W. Müller 1912

Acanthocypris bicuspis var. mucronata Claus, 1892

Strandesia bornemiszai Klie, 1935: AT, OL

Nom. nov. pro Strandesia kraepelini (Daday, 1910) nec G.W. Müller, 1906

Strandesia botosaneanui Broodbakker, 1983: NT

Strandesia brteki Rybecki, 1988: PA

Strandesia canadensis (Sars, 1926) Marmonier \& Ward 1990: NA

Prionocypris canadensis Sars, 1926

Strandesia carteri Klie, 1930: NT

Strandesia caudata Klie, 1939: AT, PA

Strandesia cavernicola Broodbakker, 1983: NT

Strandesia chondropherusa Rome, 1965: AT

Strandesia complexa Victor \& Fernando, 1981: OL

Strandesia cyprinotoides Klie, 1938: AT

Strandesia diversicolor Klie, 1938: AT

Strandesia donnetii (Baird, 1850) Martens \& Behen 1994: NT

Cypris donnetii Baird, 1850

Strandesia dorsolonga Rome, 1962: AT

Strandesia dorsoviridis McKenzie, 1966: AU 
Strandesia elatior (Vávra, 1897) Savatenalinton \& Martens 2009b: AT

Cypris elatior Vávra, 1897

Strandesia elliptica (Sars, 1901) G.W. Müller 1912: NT

Neocypris elliptica Sars, 1901

Strandesia elliptica elliptica (Sars, 1901)

Strandesia elliptica mayor Roessler, 1990

Strandesia elongata Hartmann, 1964: OL

Strandesia evae Gauthier, 1951: AT

Strandesia ewaldi Karanovic, 2006: NT

Nom. nov. pro Strandesia labiata Roessler, 1990 nec Hartmann, 1964

Strandesia feuerborni Klie, 1932: OL

Strandesia flavescens Klie, 1932: OL, PA

Strandesia freyi Victor \& Fernando, 1981: OL

Strandesia gibbosa (Baird, 1838) comb. nov.: OL, PA

Cypris gibbosa Baird, 1838

Sataracypris gibbosa (Baird, 1938) Deb 1983

Strandesia gopinathani George \& Martens, 1993: OL

Strandesia hancocki (Lowndes, 1931) Klie 1938: AT

Cyprinotus hancocki Lowndes, 1931

Strandesia hartmanni Victor et al., 1980: OL

Strandesia hornei Savatenalinton \& Martens, 2010: OL

Strandesia hulkopensis nom. nov.: OL

Nom. nov. pro Strandesia hartmanni Mannikeri \& Vaidya, 1990 nec Victor et al., 1980

Strandesia hystrix (Furtos, 1933) Martens \& Savatenalinton 2011: NA

Eucypris hystrix Furtos, 1933

Strandesia indica Hartmann, 1964: OL

Strandesia inornata (Sars, 1901) Roessler 1990: NT

Cypris inornata Sars, 1901

Strandesia intrepida Furtos, 1936: NT

Strandesia kilimensis (Daday, 1910) Klie 1935: AT

Eucypris kilimensis Daday, 1910

Strandesia kimberleyi Karanovic, 2005: AU

Strandesia kraepelini (G.W. Müller, 1906) G.W. Müller 1912: OL

Cypris kraepelini G.W. Müller, 1906

Strandesia labiata Hartmann, 1964: OL

Strandesia lansactohai Higuti \& Martens, 2013: NT

Strandesia laticauda (Daday, 1910) Klie 1935: AT

Eucypris laticauda Daday, 1910

Strandesia longiforma (Dobbin, 1941) comb. nov.: NA

Prionocypris longiforma Dobbin, 1941

Strandesia longula Broodbakker, 1983: NT

Strandesia madhuriae Harshey \& Shrivastav, 1987: OL

Strandesia marmorata (Brady, 1886) Neale 1977: OL

Cypridopsis marmorata Brady, 1886

Strandesia martensi Savatenalinton, 2015: OL

*Strandesia mercatorum (Vávra, 1895) G.W. Müller 1912: AT

Cypris (Strandesia) mercatorum Vávra, 1895

Strandesia minuta Klie, 1936: AT

Strandesia mutica (Sars, 1901) G.W. Müller 1912: NT

Neocypris mutica Sars, 1901 
Strandesia nupelia Higuti \& Martens, 2013: NT

Strandesia obliqua Klie, 1940: NT

Strandesia obtusata (Sars, 1901) G.W. Müller 1912: NT, OL

Strandesia obtusata obtusata (Sars, 1901)

Neocypris obtusata Sars, 1901

Syn.: Strandesia itapeva Tressler, 1950 (fide Martens \& Behen 1994)

Strandesia obtusata roessleri Martens \& Behen, 1994

Nom. nov. pro S. obtusata colombiensis Roessler, 1990 nec S. psittacea colombiensis Roessler, 1990

Strandesia odiosa (Moniez, 1892) Victor \& Fernando 1979a: OL

Cypris odiosa Moniez, 1892

Strandesia ovalis Tressler, 1950: NT

Strandesia perakensis Victor \& Fernando, 1981: OL

Strandesia phoenix De Deckker, 1981: AU

Strandesia pholpunthini Savatenalinton, 2015: OL

Strandesia pistrix Broodbakker, 1983: NT

Strandesia postica Rome, 1962: AT

Strandesia prava Klie, 1935: AT

Strandesia psittacea (Sars, 1901) Roessler 1990: NT

Strandesia psittacea psittacea (Sars, 1901)

Cypris psittacea Sars, 1901

Strandesia psittacea colombiensis Roessler, 1990

Strandesia puncticulata (Daday, 1910) Savatenalinton \& Martens 2009a: AT

Eucypris puncticulata Daday, 1910

Strandesia purpurascens (Brady, 1886) G.W. Müller 1912: OL

Cypris purpurascens Brady, 1886

Strandesia quasirotunda Hartmann \& Petersen, 1985: OL

Nom. nov. pro Strandesia rotunda Hartmann, 1964 nec Tressler, 1949

Strandesia regularis Rome, 1962: AT

Strandesia reticulata (Daday, 1898) G.W. Müller 1912: NA, OL, PA

Cypricercus reticulata Daday, 1898

Strandesia riograndensis Tressler, 1950: NT

Strandesia rotunda (Tressler, 1950) McKenzie 1982: NT

Cypricercus rotundus Tressler, 1950

Strandesia rouxi Méhes, 1939: NT, PAC

Strandesia saetosa Hartmann, 1964: OL

Strandesia sanoamuangae Savatenalinton \& Martens, 2010: OL

Strandesia santaeluciae (Klie, 1935) Martens \& Behen 1994: NT

Eucypris santaeluciae Klie, 1935

Strandesia sexpunctata Klie, 1932: OL

Strandesia sphaeroidea Broodbakker, 1983: NT

Strandesia spinulosa Bronstein, 1958: PA

Strandesia stocki Broodbakker, 1983: NT

Strandesia strandesioides (G.W. Müller, 1898) G.W. Müller 1912: AT

Cypris strandesioides G.W. Müller, 1898

Strandesia sudanica Sywula, 1970: PA

Strandesia taeniata (Vávra, 1895) G.W. Müller 1912: AT

Cypris taeniata Vávra, 1895

Strandesia tenuicauda (Brady, 1886) McKenzie 1972: AT

Cypris tenuicauda Brady, 1886

Strandesia thermalis Rybecky, 1988: PA 
MEISCH C. et al., Global checklist of extant non-marine Ostracoda

Strandesia tietensis Tressler, 1950: NT

Strandesia tolimensis Roessler, 1990: NT

Strandesia towoetensis Tressler, 1937: AU

Strandesia trichosa Roessler, 1990: NT

Strandesia ujijensis Rome, 1962: AT

Strandesia unguiculata Brehm, 1938: AT

Strandesia unicolor Klie, 1944: AT

Strandesia variegata (Sars, 1901) G.W. Müller 1912: NT

Neocypris variegata Sars, 1901

Strandesia vavrai (G.W. Müller, 1898) G.W. Müller 1912: AT, PA

Strandesia vavrai vavrai (G.W. Müller, 1898)

Cypris vavrai G.W. Müller, 1898

Strandesia vavrai persica Bronstein, 1925

Strandesia velhoi Higuti \& Martens, 2013: NT

Strandesia venezolana Broodbakker, 1983: NT

Strandesia victori Harshey \& Srinivasan, 1987: OL

Strandesia vinciguerrae (Masi, 1905) G.W. Müller 1912: AT, PA, OL, PAC

Cypris vinciguerrae Masi, 1905

Syn.: Strandesia anterotundata Rome, 1977 (fide Martens 1984)

Strandesia wierzejskii (Grochmalicki, 1915) Klie 1932: AT, OL

Cypris wierzejskii Grochmalicki, 1915

Strandesia wolterecki Tressler, 1937: OL

Tanganyikacypridopsis Martens, 1985

Tanganyikacypridopsis anomala (Rome, 1962) Martens 1985: AT

Cypridopsis anomala Rome, 1962

Tanganyikacypridopsis calcarata (Rome, 1962) Martens 1985: AT

Cypridopsis calcarata Rome, 1962

*Tanganyikacypridopsis depressa (Kiss, 1959) Martens 1985: AT

Potamocypris depressa Kiss, 1959

Tanganyikacypris Kiss, 1961

*Tanganyikacypris matthesi Kiss, 1961: AT

Tanganyikacypris stappersi Wouters et al., 1989: AT

Tanganyikacythere Ducasse \& Carbonel, 1993

* Tanganyikacythere burtonensis Ducasse \& Carbonel, 1993: AT

Syn.: Tanganyikacythere banzaensis Ducasse \& Carbonel, 1993 (fide Wouters \& Martens 1994)

Syn.: Tanganyikacythere mondegueri Ducasse \& Carbonel, 1993 (fide Wouters \& Martens 1994)

Tanganyikacythere caljoni Wouters \& Martens, 1994: AT

Tanganyikacythere fulgens Wouters \& Martens, 2007: AT

Tanganyikacythere rotunda Wouters \& Martens, 2008: AT

Tanycypris Triebel, 1959

Tanycypris alfonsi Nagler et al., 2014: PA

Tanycypris centa Chang et al., 2012: PA

Tanycypris eugenkempfi Savatenalinton, 2017: OL

*Tanycypris madagascarensis (G.W. Müller, 1898) Triebel 1959: AT

Cypris madagascarensis G.W. Müller, 1898

Tanycypris pellucida (Klie, 1932) Victor \& Fernando 1981: OL, PA

Dolerocypris pellucida Klie, 1932 
Syn.: Strandesia camaguinensis Tressler, 1937 (fide Victor \& Fernando 1981)

Tanycypris pellucida var. saloica (Akatova, 1950) Martens \& Savatenalinton 2011

Dolerocypris pellucida var. saloica Akatova, 1950

Tanycypris siamensis Savatenalinton \& Martens, 2009: OL

Terrestricandona Danielopol \& Betsch, 1980

*Terrestricandona minuta Danielopol \& Betsch, 1980: AT

Terrestricypris Schornikov, 1980

*Terrestricypris arborea Schornikov, 1980: PA, PAC

Terrestricypris wurdigae Pinto et al., 2005: NT

Terrestricythere Schornikov, 1969

Terrestricythere elisabethae Horne et al., 2004: PA

*Terrestricythere ivanovae Schornikov, 1969: PA

Terrestricythere pratensis Schornikov, 1980: PA

Terrestricythere proboscidis Hiruta et al., 2007: PA

Thalassocypria Hartmann, 1957

*Thalassocypria aestuarina Hartmann, 1957: NT

Thalassocypria elongata Hartmann, 1957: NT

Thalassocypria sarbui Maddocks, 1993: NT

Thermastrocythere Hobbs \& Walton, 1966

*Thermastrocythere riojai (Hoff, 1943) Hobbs \& Hobbs 1970: NA

Entocythere (Cytherites) riojai Hoff, 1943

Syn.: Thermastrocythere harti Hobbs \& Walton, 1966 (fide Hart \& Hart 1974)

Thermopsis Külköylüoğlu et al., 2003

*Thermopsis thermophila Külköylüoğlu et al., 2003: NA

Tonnacypris Diebel \& Pietrzeniuk, 1975

Type species: Tonnacypris loessica Diebel \& Pietrzeniuk, 1975 (fossil species)

Tonnacypris angulata (Yang, 1985) Van der Meeren et al. 2009: PA

Paracypricercus angulatus Yang, 1985

Tonnacypris convexa Diebel \& Pietrzeniuk, 1975: PA

Tonnacypris crassoides (Alm, 1914) Martens \& Savatenalinton 2011: PA

Eucypris crassoides Alm, 1914

Tonnacypris edlundi Van der Meeren et al., 2009: PA

Tonnacypris estonica (Järvekülg, 1960) Van der Meeren et al. 2009: PA

Ilyodromus estonicus Järvekülg, 1960

Tonnacypris glacialis (Sars, 1890) Griffiths 1995: NA, PA

Herpetocypis glacialis Sars, 1890

Tonnacypris glacialis var. albida (Alm, 1914) Griffiths 1995

Eucypris glacialis var. albida Alm, 1914

Tonnacypris lutaria (Koch, 1838) Diebel \& Pietrzeniuk 1975: NT, PA

Cypris lutaria Koch, 1838

Syn.: Cypris strigata O.F. Müller, 1776 (unused older synonym, see Taxonomic notes)

Syn.: Tonnacypris strigata (O.F. Müller, 1776) comb. nov.

Tonnacypris mazepovae Van der Meeren et al., 2009: PA

Tonnacypris tonnensis (Diebel \& Pietrzeniuk, 1975) Schornikov 2007: PA

Amplocypris tonnensis Diebel \& Pietrzeniuk, 1975 


\section{Trajancandona Karanovic, 1999}

*Trajancandona natura Karanovic, 1999: PA

Trajancandona particula Karanovic, 1999: PA

Trajancypris Martens, 1989

Trajancypris clavata (Baird, 1838) Martens 1989: PA

Cypris clavata Baird, 1838

Syn.: Cypris celtica Baird, 1859 (fide Martens 1989)

Syn.: Candonocypris ferdinandi Lindner, 1920 (fide Martens 1989)

Syn.: Eucypris clavata parva Bronstein, 1925 (fide Martens 1989)

Syn.: Eucypris clavata inversa Sywula, 1967 (fide Martens 1989)

Syn.: Sclerocypris clavata prisca Diebel \& Pietrzeniuk, 1969 (fide Martens 1989)

Trajancypris domingensis (Daday, 1910) Martens \& Behen 1994: NT

Eucypris domingensis Daday, 1910

Trajancypris laevis (G.W. Müller, 1900) Martens 1989: PA

Cypris laevis G.W. Müller, 1900

Syn.: *Cypris lienenklausi laevis G.W. Müller, 1900 (fide Martens 1989)

Trajancypris onusta (Masi, 1905) Martens 1989: PA

Cypris onusta Masi, 1905

Syn.: Trajancypris clavata sensu Bronstein 1925 (fide Martens 1989)

Syn.: Trajancypris serrata sensu Alm, 1915 (fide Martens 1989)

Trajancypris pugionis (Furtos, 1936) comb. nov.: NA

Candonocypris pugionis Furtos, 1936

Trajancypris serrata (G.W. Müller, 1900) Martens 1989: PA

Cypris serrata G.W. Müller, 1900

Syn.: Cypris lienenklausi serrata G.W. Müller, 1900 (fide Martens 1989)

Syn.: Eucypris serrata balcanica Petkovski, 1959 (fide Martens 1989)

Syn.: Prionocypris muelleri Danielopol \& McKenzie, 1977 (fide Martens 1989)

Nom. nov. pro Prionocypris serrata (G.W. Müller) nec Norman 1862

Trigonocypris De Deckker, 1976

Trigonocypris globulosa De Deckker, 1978: AU

*Trigonocypris timmsi De Deckker, 1976: AU

Tungucypridopsis Victor, 1983

*Tungucypridopsis lairdi (Victor \& Fernando, 1978) Victor 1983: PAC

Cypridopsis lairdi Victor \& Fernando, 1978

Typhlocypris Vejdovský, 1882

Syn.: Cypris (Typhlocypris) Vejdovský, 1882 (fide Namiotko et al. 2014)

Syn.: Cavernocandona Hartmann, 1964 (fide Namiotko et al. 2014)

Typhlocypris cavicola (Klie, 1935) Karanovic 2005: PA

Candona cavicola Klie, 1935

Syn.: Pseudocandona pretneri Danielopol, 1982 (fide Karanovic 2005)

Typhlocypris danubialis (Iepure et al., 2007) Namiotko et al. 2014: PA

Pseudocandona danubialis Iepure et al., 2007

*Typhlocypris eremita (Vejdovský, 1882) Šoštarić 1888: PA

Cypris (Typhlocypris) eremita Vejdovský, 1882 (fide Namiotko et al. 2014)

Syn.: Candona szoecsi Farkas, 1958 (fide Karanovic 2005)

Syn.: Candona pannonicola Löffler, 1960 (fide Meisch 2000)

Syn.: Cavernocandona dispar Hartmann, 1964 (fide Meisch 2000)

Typhlocypris marmonieri (Namiotko \& Danielopol, 2004) Namiotko et al. 2014: PA

Pseudocandona marmonieri Namiotko \& Danielopol, 2004 
Typhlocypris puteana (Klie, 1931) Karanovic 2005: PA

Candona puteana Klie, 1931

Typhlocypris (Typhlocypris) puteana (Klie, 1931) Karanovic 2005

Typhlocypris serbani (Danielopol, 1982) Namiotko et al. 2014: PA

Pseudocandona serbani Danielopol, 1982

Typhlocypris skadari Karanovic, 2005: PA

Typhlocypris (Typhlocypris) skadari Karanovic, 2005

Typhlocypris sywulai (Namiotko et al., 2004) Namiotko et al. 2014: PA

Pseudocandona sywulai Namiotko et al., 2004

Typhlocypris transylvanica (Iepure et al., 2007) Namiotko et al. 2014: PA

Pseudocandona transylvanica Iepure et al., 2007

Typhlocypris trigonella (Klie, 1931) Karanovic 2005: PA

Candona trigonella Klie, 1931

Typhlocypris (Typhlocypris) trigonella (Klie, 1931) Karanovic 2005

Tyrrhenocythere Ruggieri, 1955

Tyrrhenocythere amnicola (Sars, 1887) Schornikov, 1966: PA

Tyrrhenocythere amnicola amnicola (Sars, 1887) Schornikov 1966

Cythere amnicola Sars, 1887

Syn.: Cythere sicula Brady, 1902 (fide Schornikov 1981)

Syn.: *Thyrrhenocythere pignattii Ruggieri, 1955 (fide Schornikov 1981)

Tyrrhenocythere amnicola caspiensis Schornikov, 1981

Tyrrhenocythere amnicola dadayi Schornikov, 1981

Tyrrhenocythere donetziensis (Dubowsky, 1926) Krstić 1977: PA

Cythereis donetziensis Dubowsky, 1926

Ufocandona Külköylüoğlu et al., 2017

*Ufocandona hannaleeae Külköylüoğlu et al., 2017: NA

Uncinocythere Hart, 1962

Uncinocythere allenae Hart \& Hart, 1971: NA

Uncinocythere ambophora (Walton \& Hobbs, 1959) Hart 1962: NA

Entocythere ambophora Walton \& Hobbs, 1959

Uncinocythere bicuspide (Rioja, 1943) Hart 1962: NA, NT

Entocythere (Cytherites) bicuspide Rioja, 1943

Uncinocythere cassiensis Hart, 1965: NA

Uncinocythere caudata (Kozloff, 1955) Hart 1962: NA

Entocythere caudata Kozloff, 1959

Uncinocythere clemsonella (Crawford, 1961) Hart 1962: NA

Entocythere clemsonella Crawford, 1961

*Uncinocythere columbia (Dobbin, 1941) Hart 1962: NA

Entocythere columbia Dobbin, 1941

Uncinocythere cuadricuspide (Rioja, 1943) Hart 1962: NA

Entocythere cuadricuspide Rioja, 1943

Uncinocythere dobbinae (Rioja, 1943) Hart 1962: NT

Entocythere (Cytherites) dobbinae Rioja, 1943

Uncinocythere equicurva (Hoff, 1944) Hart 1962: NA

Entocythere equicurva Hoff, 1944

Syn.: Entocythere telmoecea Crawford, 1959 (fide Andolshek \& Hobbs 1986)

Syn.: Entocythere lucifugas Walton \& Hobbs, 1959 (fide Andolshek \& Hobbs 1986) 
Uncinocythere ericksoni (Kozloff, 1955) Hart 1962: NA

Entocythere ericksoni Kozloff, 1955

Uncinocythere holti Hart, 1965: NA

Uncinocythere neglecta (Westervelt \& Kozloff, 1959) Hart 1962: NA

Entocythere neglecta Westerwelt \& Kozloff, 1959

Uncinocythere occidentalis (Kozloff \& Whitman, 1954) Hart 1962: NA, PA

Entocythere occidentalis Kozloff \& Whitman, 1954

Uncinocythere pholetera (Hart \& Hobbs, 1961) Hart 1962: NA

Entocythere pholetera Hart \& Hobbs, 1961

Uncinocythere simondsi (Hobbs \& Walton, 1960) Hart 1962: NA

Entocythere simondsi Hobbs \& Walton, 1960

Uncinocythere spathe Hart \& Hart, 1971: NA

Uncinocythere stubbsi Hobbs \& Walton, 1966: NA

Uncinocythere thektura Hart, 1965: NA

Uncinocythere warreni Hobbs \& Walton, 1968: NA

Uncinocythere xania (Hart \& Hobbs, 1961) Hart 1962: NA

Entocythere xania Hart \& Hobbs, 1961

Uncinocythere xena Hart \& Hart, 1971: NA

Uncinocythere zancla Hobbs \& Walton, 1963: NA

Uncinocythere zaruri Hobbs, 1971: NT

Undulacandona Smith, 2011

Undulacandona aeolus Karanovic \& Cho, 2017: PA

Undulacandona andromeda Karanovic \& Cho, 2017: PA

Undulacandona cetus Karanovic \& Cho, 2017: PA

Undulacandona colymba Smith \& Kamiya, 2015: PA

Undulacandona koreana Karanovic \& Cho, 2017: PA

*Undulacandona spinula Smith, 2011: PA

Vestalenula Rossetti \& Martens, 1998

*Vestalenula boteai (Danielopol, 1970) Rossetti \& Martens 1998: OL, PA

Darwinula boteai Danielopol, 1970

Vestalenula botocuda Pinto et al., 2003: NT

Vestalenula carinata Pinto et al., 2013: NT

Vestalenula cornelia Smith et al., 2006: PA

Vestalenula cuneata (Klie, 1939) Rossetti \& Martens 1998: AT, PA

Darwinula cuneata Klie, 1939

Vestalenula cylindrica (Straub, 1952) Janz et al. 2001: PA

Darwinula cylindrica Straub, 1952

Vestalenula danielopoli (Martens et al., 1997) Rossetti \& Martens 1998: PA

Darwinula danielopoli Martens et al., 1997

Vestalenula daps (Harding, 1962) Rossetti \& Martens 1998: AU, PAC

Darwinula daps Harding, 1962

Vestalenula flexuosa Rossetti \& Martens, 1999: AT

Vestalenula inconspicua (Klie, 1935) Rossetti \& Martens 1998: AT Darwinula inconspicua Klie, 1935

Vestalenula irajai Pinto et al., 2003: NT

Vestalenula lundi (Neale \& Victor, 1978) Rossetti \& Martens 1998: OL, PA Darwinula lundi Neale \& Victor, 1978

Vestalenula marlieri (Kiss, 1959) Rossetti \& Martens 1998: AT

Darwinula marlieri Kiss, 1959 
Vestalenula marmonieri Rossetti \& Martens, 1999: AU, PAC

Vestalenula matildae Martens \& Rossetti, 2002: AU

Vestalenula molopoensis (Martens \& Rossetti, 1997) Rossetti \& Martens 1998: AT, OL, PA Darwinula molopoensis Martens \& Rossetti, 1997

Vestalenula pagliolii (Pinto \& Kotzian, 1961) Rossetti \& Martens 1998: NT, PA Darwinula pagliolii Pinto \& Kotzian, 1961

Waltoncythere Hobbs \& Peters, 1978

*Waltoncythere acuta (Hobbs \& Peters, 1977) Hobbs \& Peters 1978: NA Aphelocythere acuta Hobbs \& Peters, 1977

Xestoleberis Sars, 1866

Xestoleberis arcturi Triebel, 1956: NT

*Xestoleberis aurantia (Baird, 1838) Sars 1866: PA

Cythere aurantia Baird, 1838

Xestoleberis curassavica Klie, 1939: PA, NT

Xestoleberis fuscomaculata G.W. Müller, 1894: PA

Xestoleberis mixohalina Keyser, 1976: NA

Zonocypretta De Deckker, 1981

*Zonocypretta kalimna De Deckker, 1981: AU

Zonocypris G.W. Müller, 1898

Zonocypris alveolata Klie, 1936: AT

Zonocypris calcarata Klie, 1936: AT

Zonocypris cordata Sars, 1924: AT

Zonocypris corrugata Rome, 1965: AT

Zonocypris costata (Vávra, 1897) G.W. Müller 1898: AT, PA

Cypridopsis costata Vávra, 1897

Syn.: *Zonocypris madagascarensis G.W. Müller, 1898 (fide Martens 1984)

Zonocypris elegans G.W. Müller, 1898: AT

Zonocypris glabra Klie, 1944: AT

Zonocypris inconspicua Schäfer, 1952: PA

Zonocypris inornata Klie, 1936: AT

Zonocypris laevis Sars, 1910: AT

Zonocypris lata Rome, 1962: AT

Zonocypris peralta Rome, 1969: AT

Zonocypris pilosa Rome, 1962: AT

Zonocypris tuberosa G.W. Müller, 1908: AT

Zonocypris uniformis Rome, 1962: AT

\section{Species excluded from the alphabetic checklist}

Species excluded from the alphabetic checklist by the taxonomic filter (Müller 1912; see 'Material and methods' for more details), neither reassessed nor redescribed after 1912. Additional dubious species, insufficiently described and therefore currently unrecognizable, often unrecorded since their first description, and nomina nuda are also included.

Argentocypris labyrinthica (Cusminsky \& Whatley, 1996) Díaz \& Martens 2014: NT

Eucypris labyrinthica Cusminsky \& Whatley, 1996

Described from juveniles (fide Díaz \& Martens 2014). 
Argentocypris virgata (Cusminsky \& Whatley, 1996): NT

Eucypris virgata Cusminsky \& Whatley, 1996

Described from juveniles (fide Díaz \& Martens 2014).

Candona albida (Dana, 1849) Dana 1852: NT

Cypris albida Dana, 1849

Müller 1912: 234, under Cypridarum genera dubia et species dubiae.

Not a Candona, because Dana (1852) illustrated antennal natatory setae, the latter being absent from all species of the subfamily Candoninae.

Candona bertrandi (Margalef, 1958) Löffler 1967: PA

Typhlocypris bertrandi Margalef, 1958

Insufficiently described, unrecorded since first described. Possibly a synonym of either Candona neglecta Sars, 1890 or Candona lindneri Petkovski, 1969.

Candona claudiopolitana (Daday, 1900) G.W. Müller 1912: PA

Eucandona claudiopolitana Daday, 1900

Insufficiently described.

Candona claviformis (Brady \& Norman, 1889) Norman \& Brady 1909: PA

Candona candida var. claviformis Brady \& Norman, 1889

Müller 1912: 148, listed under Candona sp. Not described.

Not in the faunal synopses of Henderson (1990) and Meisch (2000).

Candona eleonorae Ponyi, 1956: PA

Insufficiently described, possibly identical to Neglecandona neglecta (Sars, 1887).

Candona granulata Daday, 1893: PA

Müller 1912: 135, marked with a '?', and treated as a synonym of Candona candida.

Candona hungarica (Daday, 1900) G.W. Müller 1912: PA

Eucandona hungarica Daday, 1900

Unrecognizable species, possibly identical to Pseudocandona marchica (Hartwig, 1899).

Candona lactea Baird, 1850: PA

Müller 1912: 147, larva?

Candona lucens (Baird, 1835) Baird 1850: PA

Cypris lucens Baird, 1835

Müller 1912: 135, listed under Candona candida as ?1850 C. lucens, W. Baird.

Candona similis Baird, 1845: PA

Müller 1912: 148, larva?

Candona zetlandica (Brady, 1868) Norman \& Brady 1909: PA

Cytheridea zetlandica Brady, 1868

Müller 1912: 135, listed under Candona candida as ?1868 Cytheridea zetlandica G.S. Brady.

Chlamydotheca bennelong (King, 1855): AU

Cypris bennelong King, 1855

Müller 1912: 221, juvenile?

Chlamydotheca brasiliensis (Lubbock, 1855) Brady 1886b: NT

Cypris brasiliensis Lubbock, 1855

Müller 1912: 235, listed under Cypridarum genera dubia and species dubiae.

Cyclocypris castanea Brady, 1913: AT

Insufficiently described. Unrecorded since first described.

Cyclocypris pantherina (Fischer, 1851) Kaufmann 1900: PA

Cypris pantherina Fischer, 1851

Müller 1912: 127-128, = Cyclocypris laevis (partim); = Cyclocypris ovum (partim).

Cyclocypris scutigera (Fischer, 1851) Kaufmann 1900: PA

Cypris scutigera Fischer, 1851

Müller 1912: 127-128, = Cyclocypris laevis (partim); = Cyclocypris ovum (partim). 
Cypria lepidula (Koch, 1837) Brady \& Norman 1889: PA

Cypris lepidula Koch, 1837

Müller 1912: 127-128, as a syn. with a '?' under both Cyclocypris laevis and Cyclocypris ovum.

Unrecorded since 1837.

Cypridopsis salina (Brady, 1907) G.W. Müller 1912: AT

Proteocypris salina Brady, 1907

Accepted by Müller 1912, but unrecorded since 1907, not in Martens (1984).

Cypricercus affinis Daday, 1900: AU (New Guinea)

Müller 1912: 220, accepted species, but unrecorded since 1900.

Cypridopsis hartmanni Purasjoki, 1981: PA

Nom. nud., fide Kempf 1997a.

Cypridopsis werneri Brehm, 1928: PA

Uncertain species, insufficiently described. Unrecorded since 1928.

Cypridopsis yallahensis (Baird, 1862) Daday 1905: NT

Cypris yallahensis Baird, 1862

Uncertain species; incertae sedis, fide Martens \& Behen 1994.

Cyprinotus americanus Cushman, 1905: NA

Müller 1912: 220, insufficiently described and unrecorded since first described.

Cypris altissima Chambers, 1877: NA

Müller 1912: 220, insufficiently described and unrecorded since first described.

Cypris joanna Baird, 1835: PA

Müller 1912: 236, insufficient description of a known species?

Cypris kumari (Arora, 1931) McKenzie 1972: OL

Eurycypris kumari Arora, 1931

Uncertain species, insufficiently described.

Cypris matthaii (Arora, 1931) McKenzie 1972: OL

Eurycypris matthaii Arora, 1931

Uncertain species, insufficiently described.

Cypris protubera Victor \& Fernando, 1978: OL

Uncertain species, insufficiently described.

Cypris puertoricoensis Ferguson, 1967: NT

Uncertain species, insufficiently described.

Cypris rubida Zaddach, 1844: PA

Müller 1912: 229, insufficient description of a known species?

Cypris tessellata Fischer, 1851: PA

Müller 1912: 231, insufficient description; juvenile?

Cypris vitiensis Dana, 1852: PAC

Müller 1912: 233, uncertain species. Unrecorded since its original description.

Darwinula managuensis Swain \& Gilby, 1965: NT

Rossetti \& Martens 1998: insufficiently described, doubtful species.

Darwinula yaquensis Swain, 1967: NA

Rossetti \& Martens 1998: insufficiently described, doubtful species.

Entocythere insignipes (Sars, 1926) Hoff 1942: NA

Cytherites insignipes Sars, 1926

Unrecorded since first described. Listed under 'Incertae sedis' in Williams \& Weaver (2018).

Eucypris gibberula (Koch, 1838) Daday 1900: PA

Cypris gibberula Koch, 1838

Müller 1912: 236, juvenile? Insufficient description of a known species?

Described from Germany, but not listed in Klie (1938). 
Eucypris halyi (Brady, 1886) Daday 1910: AT, OL

Cypris halyi Brady, 1886

Müller 1912: 224, insufficiently described. With a '?' in Martens (1984).

Eucypris unifasciata (Jurine, 1820) Daday 1900: PA

Monoculus unifasciatus Jurine, 1820

Kaufmann 1900: 213, an unrecognizable species.

Müller 1912: 232, insufficient description of a known species? Juvenile?

Fabaeformiscandona csikii (Daday, 1901) Altınsaçlı \& Griffiths 2002: PA

Eucandona csikii Daday, 1901

Insufficiently described, not recognizable with certainty.

Fabaeformiscandona detecta (O.F. Müller, 1776) comb. nov.: PA

Cypris detecta O.F. Müller, 1776

Possibly identical with $F$. protzi; might be a juvenile.

Goniocypris triangulata Huang, 1983: PA

Uncertain species.

G.W. Müller 1912: 398, on G. mitra Brady \& Robertson, 1870: type species of the genus.

Goniocypris is a larva of a species of Anodonta (Unionidae, Mollusca).

Hemicypris kagawaensis Okubo, 2004: PA

Hemicypris kawagaensis Okubo, 2004 (misspelling)

Nom. nud., no designation of type material.

Hemicypris okayamensis Okubo, 2004: PA

Hemicypris okayamenesis Okubo, 2004 (misspelling)

Nom. nud., no designation of type material.

Herpetocypris lucida (Koch, 1838) Brady \& Norman 1889: PA

Cypris lucida Koch, 1838

Müller 1912: 236, insufficient description of a known species?

Herpetocypris minnesotensis (Herrick, 1887) Turner 1895: NA

Cypris minnesotensis Herrick, 1887

Müller 1912: 226, uncertain species and generic assignment. A species of Dolerocypris?

Herpetocypris telavivensis Krampner, 1928: PA

Insufficiently described, with uncertain generic assignment.

Unrecorded since 1928.

Herpetocypris testudinaria Cushman, 1908: NA

Müller 1912: 231, uncertain genus and species.

Unrecorded since its first description.

Ilyocypris bistrigata (Jurine, 1820) Brady \& Norman 1889: PA

Monoculus bistrigatus Jurine, 1820

Müller 1912: 154, listed with a '?' as a syn. of I. bradyi.

Kaufmann 1900: 343, listed with a '?' as a syn. of I. gibba.

Unrecorded since 1900.

Ilyocypris bituberculata (Costa, 1847) Masi 1906: PA

Cypris bituberculata Costa, 1847

Insufficiently described, unrecorded since 1847.

Ilyocypris nipponica Okubo, 2004: PA

Nom. nud., no designation of type material.

Ilyocypris sinuata (Fischer, 1848) Brady \& Norman 1889: PA

Cypris sinuata Fischer, 1848

Müller 1912: 154, listed with a '?' as a syn. of I. bradyi.

Kaufmann 1900: 23, unidentifiable.

Unrecorded since 1900. 
Ilyocypris strausii (Plateau, 1868) G.W. Müller 1912: PA

Cypris strausii Plateau, 1868

Müller 1912: 154, listed with a '?' in the synonymy of Ilyocypris bradyi.

Unrecorded since 1900.

Plesiocypridopsis virescens (Brady, 1902) Rome 1965: PA

Cypridopsis (Candonella) virescens Brady, 1902

Müller 1912: 194, with a '?' in the list of synonyms of Herpetocypris reptans; juvenile?

Potamocypris helicina (Fischer, 1847) Daday 1900: PA

Cypris helicina Fischer, 1847

Müller 1912: 224, insufficient description of a known species?

Pseudocandona dichtliae (Brehm, 1953) Meisch 2000: PA

Candona dichtliae Brehm, 1953

Insufficiently described and unrecognizable species (fide Meisch 2000).

Pseudocandona ruttneri (Brehm, 1953) Martens \& Savatenalinton 2011: PA

Candona ruttneri Brehm, 1953

Insufficiently described and unrecognizable species (fide Meisch 2000).

Strandesia marina Hartmann, 1965: NA

Insufficiently described and unrecorded since first described.

Strandesia tonolli Moroni, 1961: PA

Nom. nud. (fide Pieri et al. 2015).

\section{Acknowledgements}

The present list is largely an expanded version of the one presented by Martens \& Savatenalinton (2011). The latter author is here acknowledged for her contribution to this earlier version of the present list. We are grateful to Tadeusz Namiotko and an anonymous reviewer for their very helpful comments and suggestions. Alain Faber, head of the National Natural History Museum of Luxembourg, is thanked for his constant support of the first author's (CM) research activities. We thank Danny Eibye-Jacobsen and Kristiaan Hoedemakers of EJT for their much-appreciated comments and suggestions for improvement of the manuscript.

\section{References}

Alm G. 1915. Monographie der schwedischen Süsswasser-Ostracoden nebst systematischen Besprechungen der Tribus Podocopa. Zoologiska Bidrag från Uppsala 4: 1-248.

Altınsaçl1 S. \& Griffiths H.I. 2002. A review of the occurrence and distribution of the Recent non-marine Ostracoda (Crustacea) of Turkey. Zoology in the Middle East 27: 61-76. https://doi.org/10.1080/09397140.2002.10637941

Andolshek M.D. \& Hobbs H.H. 1986. The entocytherid ostracod fauna of southeastern Georgia. Smithsonian Contributions to Zoology 424: 1-43.

Athersuch J. 1982. Some ostracod genera formerly of the Family Cytherideidae Sars. In: Bate R.H., Robinson E. \& Sheppard L.M. (eds) Fossil and Recent Ostracods: 231-275. Ellis Horwood, Chichester.

Athersuch J., Horne D.J. \& Whittaker J.E. 1989. Marine and Brackish Water Ostracods. Synopses of the British Fauna (New Series) 43. E.J. Brill, London.

Baird W. 1845. Arrangement of the British Entomostraca, with a list of species, particularly noticing those which have as yet been discovered within the bounds of the Club. Transactions of the Berwickshire Naturalists' Club 2 (13): 145-158. 
MEISCH C. et al., Global checklist of extant non-marine Ostracoda

Balian E.V., Segers H., Lévêque C. \& Martens K. 2008. An introduction to the freshwater animal diversity assessment (FADA) project. Hydrobiologia 595: 3-8. https://doi.org/10.1007/978-1-4020-8259-7_1

Baltanás A. 2001. Candelacypris n. gen. (Crustacea, Ostracoda): a new genus from Iberian saline lakes, with a redescription of Eucypris aragonica Brehm \& Margalef, 1948. Bulletin de la Société des Naturalistes luxembourgeois 101: 183-192.

Baltanás A., Beroiz B. \& López A. 1996. Lista faunistica y bibliográfica de los ostrácodos no-marinos (Crustacea, Ostracoda) de la Península Ibérica, Islas Baleares e Islas Canarias. Listas de la flora y fauna de las aguas continentales de la peninsula ibérica 12, Asociación Española de Limnología.

Barclay M.H. 1968. Additions to the freshwater ostracod fauna of New Zealand. New Zealand Journal of Marine and Freshwater Research 2: 67-80.

Bate R.H. 1970. A new species of Hemicypris (Ostracoda) from ancient beach sediments of Lake Rudolf, Kenya. Palaeontology 13 (2): 289-296.

Bate R.H. 1972. Fossil and living Hemicypris (Ostracoda) from Lake Rudolf, Kenya. Palaeontology 15: $184-185$.

Bates M.R., Keen D.H., Whittaker J.E., Merry J.S. \& Wenban-Smith F.F. 2002. Middle Pleistocene molluscan and ostracod faunas from Allhallows, Kent, UK. Proceedings of the Geologists' Association 113: 223-236. https://doi.org/10.1016/S0016-7878(02)80026-8

Benson R.H. \& Kaesler R.L. 1963. Recent marine and lagoonal ostracodes from the Estero de Tastiota region, Sonora, Mexico (northeastern Gulf of California). The University of Kansas Paleontological Contributions, Arthropoda 3: 1-34.

Benson R.H. \& McDonald H.C. 1963. Postglacial (Holocene) ostracodes from Lake Erie. University of Kansas Paleontological Contributions 4: 1-26.

Blake C.H. 1931. Two freshwater ostracods from North America. Bulletin of the Museum of Comparative Zoölogy at Harvard College 72 (7): 279-292.

Bode S.N.S., Adolfsson S., Lamatsch D.K., Martins M.J.F., Schmit O., Vandekerkhove J., Mezquita F., Namiotko T., Rossetti G., Schön I., Butlin R.K. \& Martens K. 2010. Exceptional cryptic diversity and multiple origins of parthenogenesis in a freshwater ostracod. Molecular Phylogenetics and Evolution 54: 542-552. https://doi.org/10.1016/j.ympev.2009.08.022

Brady G.S. 1870. Notes on Entomostraca taken chiefly in the Northumberland and Durham District (1869). Natural History Transactions of Northumberland and Durham 3 (1868-1870): 361-373.

Brady G.S. 1886a. Notes on freshwater Entomostraca from South Australia. Proceedings of the Zoological Society of London 1886 (1): 82-93.

Brady G.S. 1886b. Notes on Entomostraca collected by Mr. A. Haly in Ceylon. Journal of the Linnean Society of London, Zoology 19 (114): 293-317.

Brady G.S. \& Norman A.M. 1889. A monograph of the marine and freshwater Ostracoda of the North Atlantic and of North-Western Europe. Section I. Podocopa. The Scientific Transactions of the Royal Dublin Society, ser. 2, 4 (2): 63-270.

Brehm V. 1932. Notizen zur Süßwasserfauna Guatemalas und Mexikos. Zoologischer Anzeiger 99: 63-66.

Brehm V. 1939. La fauna microscópica del Lago Petén, Guatemala. Anales de la Escuela Nacional de Ciencias Biológicas 1: 173-203.

Brehm V. 1953. Candona dichtliae nov. spec., eine neue Ostracodenspezies. Anzeiger der Österreichischen Akademie der Wissenschaften, mathematisch-naturwissenschaftliche Klasse 90 (2): 21-22. 
Bronstein Z.S. 1928. Zur Kenntnis der Ostracoden des Kaukasus und Persiens. Travaux de la station biologique du Caucase du Nord 2 (2/3): 67-119.

Bronstein Z.S. 1947. Fauna of the USSR, Crustaceans Volume II, Number I, Fresh-water Ostracoda. Academy of Sciences of the USSR, Zoological Institute, Moscow. [In Russian.] (English translation: Oxonian Press Pvt, Ltd., New Delhi. Reprint published by the U.S. Department of Commerce, National Technical Information Service, Springfield, Virginia).

Broodbakker N.W. 1982. The genus Heterocypris (Crustacea, Ostracoda) in the West Indies. Part I. Taxonomic characters. Bijdragen tot de Dierkunde 52: 207-227.

Broodbakker N.W. 1983a. The subfamily Candoninae (Crustacea, Ostracoda) in the West Indies. Bijdragen tot de Dierkunde 53 (2): 287-326.

Broodbakker N.W. 1983b. The genus Strandesia and other Cypricercini (Crustacea, Ostracoda) in the West Indies. Part I. Taxonomy. Bijdragen tot de Dierkunde 53 (2): 327-368.

Broodbakker N.W. 1984. The genus Tanycypris (Crustacea, Ostracoda) in the West Indies. Amsterdam Expeditions to the West Indian Islands, report 37. Bijdragen tot de Dierkunde 54 (1): 15-24.

Carbonnel G. 1969. Les ostracodes du Miocène rhodanien: Systématique, biostratigraphie, écologique, paléobiologie. Documents des laboratoires de géologie de la Faculté des Sciences de Lyon 32 (1/2), Lyon.

Chapman M.A. 1967. Ostracoda. In: Bayly I.A.E., Bishop J.A. \& Hiscock I.D. (eds) An Illustrated Key to the Genera of Crustacea of Australian Inland Waters. Newsletter of the Australian Society for Limnology, special issue.

Claus C. 1892. Beiträge zur Kenntnis der Süßwassser-Ostracoden. I. Über den Körper- und Gliedmassenbau der Cypriden nebst Bemerkungen über einzelne innere Organe derselben. Arbeiten aus dem zoologischen Institute der Universität Wien und der zoologischen Station in Triest 10 (2): 147-216.

Cohen A.S. 1986. Distribution and faunal associations of benthic invertebrates at Lake Turkana, Kenya. Hydrobiologia 141: 179-197. https://doi.org/10.1007/BF00014214

Cohuo S., Hernández M.C., Pérez L. \& Alcocer J. 2017a. Candona alchichica (Podocopida: Candonidae), a new ostracod species from saline, tropical Lake Alchichica, Mexico. Journal of Limnology 76: 68-84. https://doi.org/10.4081/jlimnol.2016.1480

Cohuo S., Macario-González L., Pérez L. \& Schwalb A. 2017b. Overview of Neotropical-Caribbean freshwater ostracode fauna (Crustacea, Ostracoda): identifying areas of endemism and assessing biogeographical affinities. Hydrobiologia 786: 5-21. https://doi.org/10.1007/s10750-016-2747-1

Colin J.-P. 1981. In: Colin J.-P. \& Danielopol D.L. (eds) Sur la morphologie, la systématique, la biogéographie et l'évolution des Ostracodes Timiriaseviinae (Limnocytheridae). Paléobiologie continentale 11 (1980): 1-52.

Crandall K.A. \& Buhay J.E. 2008. Global diversity of crayfish (Astacidae, Cambaridae, and Parastacidae - Decapoda) in freshwater. Hydrobiologia 595: 295-301. https://doi.org/10.1007/s10750-007-9120-3

Daday E. 1892. Über die Ostracoden der Umgebung von Budapest. Termeszetrajzi Füzetek 15: 286-309.

Daday E. 1898. Mikroskopische Süsswasserthiere aus Ceylon. Termeszetrajzi Füzetek 21 (Supplement).

Daday E. 1900. A Magyarországi Kagylósrákok Magánrajza. Ostracoda Hungariae. Kiadja a Magyar Tudományos Akadémia, Budapest.

Daday E. 1905. Untersuchungen über die Süsswasser-Mikrofauna Paraguays. Zoologica, OriginalAbhandlungen aus dem Gesamtgebiete der Zoologie 18 (44): 1-374. 
MEISCH C. et al., Global checklist of extant non-marine Ostracoda

Daday E. 1910. Untersuchungen über die Süsswasser-Mikrofauna Deutsch-Ost-Afrikas. Zoologica, Original-Abhandlungen aus dem Gesamtgebiete der Zoologie 23: 1-314.

Danielopol D.L. 1969. Microdarwinula n. g. et quelques remarques sur la répartition de la famille Darwinulidae Br. et Norm. (Crustacea, Ostracoda). Annales de Limnologie 4 (2) (1968): 153-174.

Danielopol D.L. 1971a. Définition de la tribu des Hartiellini nov. et remarques sur la poecilogynie de Hartiella dudichi (Ostracoda, Entocytheridae, Sphaeromicolinae). Travaux de l'Institut de Spéologie 'Emile Racovitza' 10: 189-207.

Danielopol D.L. 1971b. Quelques remarques sur le peuplement ostracodologique des eaux douces souterraines d'Europe. Bulletin du Centre de Recherches de Pau-SNPA 5 (suppl.): 179-190.

Danielopol D.L. 1973. Sur la morphologie des aesthetascs chez quelques ostracodes hypogés de la sousfamille des Candoninae (Cyprididae, Podocopida). Annales de Spéléologie 28: 233-245.

Danielopol D.L. 1980. On the carapace shape of some European freshwater interstitial Candoninae (Ostracoda). Proceedings of the Biological Society of Washington 93 (3): 743-756.

Danielopol D.L. 1981. In: Colin J.-P. \& Danielopol D.L. (eds) Sur la morphologie, la systématique, la biogéographie et l'évolution des Ostracodes Timiriaseviinae (Limnocytheridae). Paléobiologie continentale 11 (1980): 1-52.

Danielopol D.L. 1982a.Mixtacandona cottarellii n. sp., un Ostracode interstitiel de Sardaigne. Bollettino del Museo Civico di Storia Naturale di Verona 8 (1981): 419-425.

Danielopol D.L. 1982b. Nouvelles données sur les Candoninae (Ostracoda) hypogés de Roumanie et Yougoslavie. Bulletin du Muséum national d'Histoire naturelle de Paris 4e série, 4 (section A) (3/4): 369-396.

Danielopol D.L. 1986. Ostracoda, part I: Stygobiont Ostracoda from inland subterranean waters. In: Botoseanu L. (ed.) Stygofauna Mundi: A Faunistic, Distributional, and Ecological Synthesis of the World Fauna Inhabiting Subterranean Waters: 265-278. Brill Academic Publisher.

Danielopol D.L. \& Cvetkov L. 1979. Trois nouvelles espèces du genre Mixtacandona (Ostracoda, Cyprididae, Candoninae). Hydrobiologia 67 (3): 249-266. https://doi.org/10.1007/BF00023181

Danielopol D.L. \& Hart C.W. 1985. Notes on the center of origin and on the antiquity of the Sphaeromicolinae, with description of Hobbsiella, new genus (Ostracoda: Entocytheridae). Stygologia 1: $54-70$.

Danielopol D.L. \& Hartmann G. 1986. Ostracoda. Part 1: Stygobiont Ostracoda from inland subterranean waters. In: Botosaneanu L. (ed.) Stygofauna Mundi, a Faunistic, Distributional, and Ecological Synthesis of the World Fauna inhabiting Subterranean Waters: 265-294. E.J. Brill / Dr. W. Backhuys, Leiden.

Danielopol D.L. \& McKenzie K.G. 1977. Psychrodromus gen. n. (Crustacea, Ostracoda), with redescription of the Cypridid genera Prionocypris and Ilyodromus. Zoologica Scripta 6 (4): 301-322.

Danielopol D.L., Geiger W., Tölderer-Farmer M., Orellana C.P., Terrat M.-N. 1988. In search of Cypris and Cythere - A report of the evolutionary ecological project on limnic Ostracoda from the Mondsee (Austria). In: Hanai T., Ikeya N. \& Ishizaki K. (eds) Evolutionary Biology of Ostracoda Its Fundamentals and Applications. Proceedings of the 9th International Symposium on Ostracoda: 485-500. Shizuoka, Japan.

Danielopol D.L., Namiotko T. \& Meisch C. 2012. Marmocandona nov. gen. (Ostracoda, Candoninae), with comments on the contribution of stygobitic organisms to micropalaeontological studies. Kölner Forum für Geologie und Paläontologie 21: 13-16. 
Danielopol D.L., Namiotko T., von Grafenstein U., Fuhrmann R., Decrouy L., Gross M., Pichler M. \& Picot L. 2015. The implementation of taxonomic harmonisation for Candoninae(Ostracoda, Cypridoidea): a heuristic solution for Fabaeformiscandona tricicatricosa (Diebel and Pietrzeniuk). Geo-Eco-Marina 21: $111-158$.

Deb M. 1983. Brief descriptions of new species of Ostracoda: Crustacea from Maharashtra State (India). Records of the Zoological Survey (India) 81: 135-166.

De Deckker P. 1974. Australocypris, a new ostracod genus from Australia. Australian Journal of Zoology 22: 91-104.

De Deckker P. 1979a. Comparative morphology and review of Australian Notodromadinae Kaufmann 1900 (Crustacea: Ostracoda). Senckenbergiana biologica 59 (1978) (5/6): 417-463.

De Deckker P. 1979b. Ostracods from the Mound Springs area between Strangways and Curdimurka, South Australia. Transactions of the Royal Society of South Australia 103 (6): 155-168.

De Deckker P. 1981. Ostracoda from Australian inland waters - Notes on taxonomy and ecology. Proceedings of the Royal Society of Victoria 93 (1): 43-85.

De Deckker P. \& McKenzie K.G. 1981. Bennelongia, a new cyprididid ostracod genus from Australasia. Transactions of the Royal Society of South Australia 105 (2): 53-58.

Delorme L.D. 1968. Pleistocene freshwater Ostracoda from Yukon, Canada. Canadian Journal of Zoology 46 (5): 859-876. https://doi.org/10.1139/z68-123

Delorme L.D. 1970. Freshwater ostracodes of Canada. Part III. Family Candonidae. Canadian Journal of Zoology 48 (5): 1099-1127. https://doi.org/10.1139/z70-194

Delorme L.D. 1971. Freshwater ostracodes of Canada. Part V. Families Limnocytheridae, Loxoconchidae. Canadian Journal of Zoology 49: 43-64. https://doi.org/10.1139/z71-009

Devoto G. 1965. Lacustrine Pleistocene in the lower Liri Valley (Southern Latium). Estratto da Geologica Romana 4: 291-368.

Díaz A.R. \& Martens K. 2014. On Argentocypris sara gen. nov., sp. nov. (Ostracoda) from the Patagonian wetlands of Argentina. Crustaceana 87: 513-530. https://doi.org/10.1163/15685403-00003300

Diebel K. \& Pietrzeniuk E. 1969. Ostracoden aus dem Mittelpleistozän von Süßenborn bei Weimar. Paläontologische Abhandlungen, Abt. A 3 (3/4): 463-488.

Diebel K. \& Pietrzeniuk E. 1975. Neue Ostracoden aus dem Pleistozän von Burgtonna (Bezirk Erfurt). Tonnacypris gen. n. und Amplocypris tonnensis sp. n. Zeitschrift für geologische Wissenschaften 3: 87-97.

Diebel K. \& Pietrzeniuk E. 1978. Die Ostrakodenfauna aus den jungpleistozänen (weichselkaltzeitlichen) Deckschichten von Burgtonna in Thüringen. Quartärpaläontologie 3: 207-221.

Dobbin C.N. 1941. Fresh-water Ostracoda from Washington and other western localities. University of Washington Publications in Biology 4: 175-246.

Dobbin Evenson C. 1969. Designation of lectotypes of fresh-water species described by Dobbin, 1941 (Ostracoda, Crustacea). In: Neale J.W. (ed.) The Taxonomy, Morphology and Ecology of Recent Ostracoda: 491-494. Oliver \& Boyd, Edinburgh.

Eagar S.H. 1971. A checklist of the Ostracoda of New Zealand. Journal of the Royal Society of New Zealand 1: 53-64.

Escrivà A., Smith R.J., Aguilar-Alberola J.A., Kamiya T., Karanovic I., Rueda J., Schornikov E.E. \& Mesquita-Joanes F. 2012. Global distribution of Fabaeformiscandona subacuta: an exotic 
MEISCH C. et al., Global checklist of extant non-marine Ostracoda

invasive Ostracoda on the Iberian Peninsula. Journal of Crustacean Biology 32 (6): 949-961. https://doi.org/10.1163/1937240X-00002096

Farkas H. 1958. Kagylósrákok - Ostracoda. Fauna Hungariae - Magyarorszag Allatvilaga 39 (4. Kötet, 3. Füzet): 1-68.

Ferguson Jr. E., Hutchinson G.E. \& Goulden C.E. 1964. Cypria petenensis, a new name for the ostracod Cypria pelagica Brehm 1932. Postilla, Peabody Museum of Natural History, Yale University 80: 1-4.

Fox H.M. 1962. On Notodromas persica (Crustacea, Ostracoda) in Italy and in France. Memorie dell'Istituto Italiano di Idrobiologia 15: 169-173.

Fox H.M. 1964. On the larval stages of cyprids and on Siphlocandona (Crustacea, Ostracoda). Proceedings of the Zoological Society of London 142 (1): 165-176.

Freels D. 1980. Limnische Ostrakoden aus dem Jungtertiär und Quartär der Türkei. Geologisches Jahrbuch, Reihe B 39: 1-169.

Fuhrmann R. 2012. Atlas quartärer und rezenter Ostrakoden Mitteleuropas. Altenburger Naturwissenschaftliche Forschungen 15: 1-320.

Fuhrmann R. \& Goth K. 2011. Neue und weitere bemerkenswerte Ostrakoden aus dem Quartär Mitteldeutschlands. Palaeontographica, Abt. A: Palaeozoology - Stratigraphy 294: 95-201.

Fuhrmann R. \& Pietrzeniuk E. 1990. Die Aussage der Ostrakodenfauna zum Sedimentationsablauf im Interglazialbecken, zur klimatischen Entwicklung und zur stratigraphischen Stellung des Interglazials von Neumark-Nord (Geiseltal). In: Mania D., Thomae M., Litt T. \& Weber T. (eds) Neumark-Gröbern: Beiträge zur Jagd des mittelpaläolithischen Menschen 43: 161-166.

Fürstenberg S., Frenzel P., Peng P., Henkel K. \& Wrozyna C. 2015. Phenotypical variation in Leucocytherella sinensis Huang, 1982 (Ostracoda) - a proxy for palaeosalinity in Tibetan lakes. Hydrobiologia 751 (1): 55-72. https://doi.org/10.1007/s10750-014-2171-3

Furtos N.C. 1936a. On the Ostracoda from the cenotes of Yucatan and vicinity. Publications of the Carnegie Institution of Washington 457: 89-115.

Furtos N.C. 1936b. Fresh-water Ostracoda from Florida and North Carolina. The American Midland Naturalist 17 (2): 491-522.

Gauthier H. 1939. Contribution à l'étude de la faune dulçaquicole de la région du Tchad et particulièrement des branchiopodes et des ostracodes. Bulletin de l'Institut Français d'Afrique Noire 1 (1): 110-244. (Ostracoda: 110-128, 189-244, figs 12-23).

George S. \& Martens K. 1993. In: George S., Martens K. \& Nayar C.K.G. (eds) Two new species of freshwater Ostracoda of the genus Parastenocypris Hartmann, 1964 from Kerala, India. Hydrobiologia 254: 183-193. https://doi.org/10.1007/BF00014112

George S. \& Martens K. 2002. On a new species of Potamocypris (Crustacea, Ostracoda) from Chalakkudy River, Kerala (India), with a checklist of the Potamocypris-species of the world. Zootaxa 66: 1-15. https://doi.org/10.11646/zootaxa.66.1.1

Gonzalez Mozo M.E., Martens K. \& Baltanás A. 1996. A taxonomic revision of European Herpetocypris (Brady and Norman, 1889 (Crustacea, Ostracoda). Bulletin de l'Institut royal des Sciences naturelles de Belgique, Biologie 66: 96-132.

Griffiths H.I. 1995. European Quaternary freshwater Ostracoda: a biostratigraphic and palaeobiogeographic primer. Scopolia 34: 1-168. 
Griffiths H.I. \& Mount J.A. 1993. Ostracods. In: Evans J.G., Limbrey S., Mate I. \& Mount R. (eds) An environmental history of the Upper Kennet Valley, Wiltshire, for the last 10,000 years. Proceedings of the Prehistoric Society n. s. 59: 139-195.

Griffiths H.I. \& Evans J.G. 1995. An annotated check-list of British Pleistocene, Holocene and modern freshwater ostracods. Journal of Micropalaeontology 14: 59-65.

Griffiths H.I., Schwalb A. \& Stevens L.R. 2001. Environmental change in southwestern Iran: the Holocene ostracod fauna of Lake Mirabad. The Holocene 11 (6): 757-764. https://doi.org/10.1191/09596830195771

Halse S.A. \& McRae J.M. 2004. New genera and species of 'giant' ostracods (Crustacea: Cyprididae) from Australia. Hydrobiologia 524: 1-52. https://doi.org/10.1023/B:HYDR.0000036197.03776.46

Harding J.P. 1955. The Armstrong College Zoological Expedition to Siwa Oasis (Libyan Desert) 1935: Crustacea: Branchiopoda and Ostracoda. Proceedings of the Egyptian Academy of Science 10: 58-68.

Harshey D.K. \& Victor R. 1983. On Parastenocypris biswasi (Deb, 1972) a freshwater ostracod (Crustacea: Ostracoda) from India. Mitteilungen aus dem hamburgischen zoologischen Museum und Institut 80: 177-182.

Hart C.W. 1962. A revision of the ostracods of the family Entocytheridae. Proceedings of the Academy of Natural Sciences of Philadelphia 114 (3): 121-147.

Hart D.G. \& Hart C.W. 1974. The Ostracod Family Entocytheridae. The Academy of Natural Sciences of Philadelphia, Monograph 18, Philadelphia.

Hartmann G. 1964. Asiatische Ostracoden, systematische und zoogeographische Untersuchungen. Internationale Revue der gesamten Hydrobiologie 3: 1-155.

Hartmann G. \& Petersen H. 1985. Homonyms of recent and fossil Ostracoda (Crustacea) and the introduction of three new names. Mitteilungen aus dem Hamburgischen Zoologischen Museum und Institut 82: 263-267.

Hartmann G. \& Puri H.S. 1974. Summary of neontological and paleontological classification of Ostracoda. Mitteilungen des Hamburgischen Zoologischen Museums und Instituts 70: 7-73.

Hartwig W. 1999. Candona euplectella (Robertson 1880) bildet eine selbständige Gattung. Zoologischer Anzeiger 22 (592): 309-311.

Henderson P.A. 1990. Freshwater ostracods. In: Kermack D.M. \& Barnes R.S.K. (eds) Synopses of the British Fauna (New Series) No. 43. Universal Book Services/Dr W. Backhuys, Oegstgeest, The Netherlands.

Henry M. 1923. A monograph of the freshwater Entomostraca of New South Wales. Part 3: Ostracoda. Proceedings of the Linnean Society of New South Wales 48: 267-286.

Herbst H.V. 1951. Isocypris quadrisetosa Rome (Crustacea, Ostracoda) aus der Eider. Anhang: Isocypris laskaradisi $\mathrm{n}$. sp. von der Insel Milos. Abhandlungen des naturwissenschaftlichen Vereins zu Bremen 32 (3): 403-414.

Higuti J. \& Martens K. 2012a. On a new cypridopsine genus (Crustacea, Ostracoda, Cyprididae) from the Upper Paraná River Floodplain (Brazil). Zootaxa 3391: 23-38.

Higuti J. \& Martens K. 2012b. Description of a new genus and species of Candonopsini (Crustacea, Ostracoda, Candoninae) from the alluvial valley of the Upper Paraná River (Brazil, South America). European Journal of Taxonomy 33: 1-31. https://doi.org/10.5852/ejt.2012.33

Hirschmann N. 1912. Beitrag zur Kenntnis der Ostrakodenfauna des Finnischen Meerbusens. Zweite Mitteilung. Acta Societatis pro Fauna et Flora Fennica 36 (2): 1-68. 
Hobbs H.H. 1966. An illustrated key to the species of the genus Ankylocythere with a description of a new species from Louisiana (Ostracoda, Entocytheridae). Proceedings of the Louisiana Academy of Sciences 29: 67-75.

Hobbs H.H. \& Hart C.W. Jr. 1966. On the entocytherid ostracod genera Ascetocythere, Plectocythere, Phymocythere (gen. nov.), and Cymocythere, with descriptions of new species. Proceedings of the Academy of Natural Sciences of Philadelphia 118 (2): 35-61.

Hobbs H.H. \& Hobbs H.H. III 1970. New entocytherid ostracods with a key to the genera of the subfamily Entocytherinae. Smithsonian Contributions to Zoology 47: 1-19.

Hobbs H.H. \& Peters D.J. 1977. The entocytherid ostracods of North Carolina. Smithonian Contributions to Zoology 247: 1-73.

Hobbs H.H. Jr. \& Peters D.J. 1978. A substitute name for the homonym Aphelocythere Hobbs and Peters (Ostracoda, Entocytheridae). Proceedings of the Biological Society of Washington 91 (4): 1037.

Hoff C.C. 1942. The Ostracods of Illinois: their Biology and Ecology. Illinois Biological Monographs 19 (1-2), Urbana, Illinois.

Hollwedel W. \& Scharf B.W. 1996. Zur Verbreitung der Cladoceren und Ostracoden (Crustacea) in Gewässern des Müritz-Nationalparks (Mecklenburg-Vorpommern). Braunschweiger Naturkundliche Schriften 5 (1): 91-104.

Horne D.J., Jocque M., Brendonck L. \& Martens K. 2011. On Potamocypris compressa (Crustacea, Ostracoda) from temporary rock pools in Utah, USA, with notes on the taxonomic harmonisation of North American and European ostracod faunas. Zootaxa 2793: 35-46.

Janz H., Jellinek T. \& Hamedani A. 2001. Holozäne Süßwasser-Ostracoden aus dem Iran. Senckenbergiana lethaea 81: 181-205.

Jones T.R. 1857. A monograph of the Tertiary Entomostraca of England. Monographs of the Palaeontographical Society 9, Palaeontographical Society, London. https://doi.org/10.5962/bhl.title.46366

Karanovic I. 1999. On Pseudocypridopsis n. gen., with a redescription of Pseudocypridopsis clathrata (Klie, 1936) and a first description of the male (Ostracoda, Cypridopsinae). Bulletin Zoölogisch Museum Universiteit van Amsterdam 17: 1-6.

Karanovic I. 2004. Towards a revision of Candoninae (Crustacea: Ostracoda): on the genus Candonopsis Vávra, with descriptions of new taxa. Subterranean Biology 2: 91-108.

Karanovic I. 2005. On the genus Typhlocypris Vejdovský, 1882 (Crustacea: Ostracoda: Candoninae), with description of two new species. Systematics and Biodiversity 3: 375-406. https://doi.org/10.1017/s1477200005001738

Karanovic I. 2006. Recent Candoninae (Crustacea, Ostracoda) of North America. Records of the Western Australian Museum, Supplement 71: 1-75. https://doi.org/10.18195/issn.0313-122x.71.2006.001-075

Karanovic I. 2007. Candoninae (Ostracoda) from the Pilbara region in Western Australia. Crustaceana Monographs 7: 1-433. Brill, Leiden, Boston. https://doi.org/10.1163/ej.9789004156937.i-434

Karanovic I. 2008. Three interesting Cyprididae (Ostracoda) from Western Australia. Records of the Western Australian Museum 24: 267-287.

Karanovic I. 2009. Four new species of Gomphodella De Deckker, with a phylogenetic analysis and a key to the living representatives of the subfamily Timiriaseviinae (Ostracoda). Crustaceana 82 (9): 1133-1176. https://doi.org/10.1163/156854009X452731

Karanovic I. 2011. On the recent Cyclocypridinae (Podocopida, Candonidae) with description of two new genera and one new species. Zootaxa 2820: 1-61. 
Karanovic I. 2012. Recent Freshwater Ostracods of the World, Crustacea, Ostracoda, Podocopida. Springer, Heidelberg/Dordrecht/London/New York.

Karanovic I. \& Datry T. 2009. Overview of Candoninae (Crustacea, Ostracoda) of South America and the West Indies, with the description of two new species and one new genus. Zootaxa 2267: 1-25.

Karanovic I. \& Marmonier P. 2002. On the genus Candonopsis (Crustacea: Ostracoda: Candoninae) in Australia, with a key to the world recent species. Annales de Limnologie 38: 199-240.

Karanovic I. \& Pesce G.L. 2000. Martenscypridopsis a new ostracod genus (Crustacea : Ostracoda) from African inland waters. International Journal of Limnology 36 (3): 149-155.

https://doi.org/10.1051/limn/2000012

Karanovic I. \& Sitnikova T.Y. 2017. Morphological and molecular diversity of Lake Baikal candonid ostracods, with description of a new genus. ZooKeys 684: 19-56.

https://doi.org/10.3897/zookeys.684.13249

Kaufmann A. 1896. Die schweizerischen Cytheriden und ihre nächsten Verwandten. Revue Suisse de Zoologie et Annales du Musée d'Histoire Naturelle de Genève 4 (2): 313-384.

Kaufmann A. 1900. Cypriden und Darwinuliden der Schweiz. Revue suisse de Zoologie 8: 209-423.

Kempf E.K. 1980a. Index and Bibliography of Nonmarine Ostracoda. 1. Index A. Sonderveröffentlichungen des Geologischen Instituts der Universität zu Köln 35: 1-188.

Kempf E.K. 1980b. Index and Bibliography of Nonmarine Ostracoda. 2. Index B. Sonderveröffentlichungen des Geologischen Instituts der Universität zu Köln 36: 1-180.

Kempf E.K. 1980c. Index and Bibliography of Nonmarine Ostracoda. 3. Index C. Sonderveröffentlichungen des Geologischen Instituts der Universität zu Köln 37: 1-204.

Kempf E.K. 1980d. Index and Bibliography of Nonmarine Ostracoda. 4. Bibliography A. Sonderveröffentlichungen des Geologischen Instituts der Universität zu Köln 38: 1-186.

Kempf E.K. 1997a. Index and Bibliography of Nonmarine Ostracoda. 6. Index A, Supplement 1. Sonderveröffentlichungen des Geologischen Instituts der Universität zu Köln 109: 1-142.

Kempf E.K. 1997b. Index and Bibliography of Nonmarine Ostracoda. 7. Index B, Supplement 1. Sonderveröffentlichungen des Geologischen Instituts der Universität zu Köln 110: 1-134.

Kempf E.K. 1997c. Index and Bibliography of Nonmarine Ostracoda. 8. Index C, Supplement 1. Sonderveröffentlichungen des Geologischen Instituts der Universität zu Köln 111: 1-152.

Kempf E.K. 1997d. Index and Bibliography of Nonmarine Ostracoda. Bibliography C. Sonderveröffentlichungen des Geologischen Instituts der Universität zu Köln 112: 1-150.

Kempf E.K. 2006. Fossil and Living Ostracoda of the World. Index and Bibliography of Nonmarine Ostracoda 16, Index D: Recent Nonmarine Ostracoda of the World. CD-ROM Edition, Cologne, Germany (published by the author).

Kempf E.K. 2015. Substitutional names and new combinations for taxa of Ostracoda (Arthropoda: Crustacea). Munis Entomology \& Zoology 10 (2): 506-517.

Keyser D. 1976. Ostracoden aus den Mangrovegebieten von Südwest-Florida (Crustacea: Ostracoda: Podocopa). Abhandlungen und Verhandlungen des naturwissenschaftlichen Vereins Hamburg, Neue Folge 18-19 (1975-1976): 255-290.

Klie W. 1929. Beitrag zur Kenntnis der Ostracoden der südlichen und westlichen Ostsee, der festländischen Nordseeküste und der Insel Helgoland. Zeitschrift für wissenschaftliche Zoologie 134 (2/3): 270-306. 
MEISCH C. et al., Global checklist of extant non-marine Ostracoda

Klie W. 1930. Ostracoden aus dem paraguayischen Teile des Gran-Chaco. Archiv für Hydrobiologie 22: 221-258.

Klie W. 1932. Die Ostracoden der deutschen limnologischen Sunda-Expedition. Archiv für Hydrobiologie, Supplementband 11: 447-502.

Klie W. 1933. Die Ostracoden der Rift-Tal-Seen in Kenia. Internationale Revue der gesamten Hydrobiologie und Hydrographie 29: 1-14.

Klie W. 1935. Die Fischereigründe vor Alexandrien. V. - Ostracoda. Notes and Memoirs of the Fisheries Research Directorate 12: 1-10.

Klie W. 1938a. Ostracoda, Muschelkrebse. Die Tierwelt Deutschlands und der angrenzenden Meeresteile nach ihren Merkmalen und nach ihrer Lebensweise. 34. Teil, Krebstiere oder Crustacea. Gustav Fischer Verlag, Jena.

Klie W. 1938b. Ostracoden aus dem Grundwasser der oberrheinischen Tiefebene. Archiv für Naturgeschichte 7: 1-28.

Klie W. 1938c. Ostracoden aus Formosa. Bulletin of the Biogeographical Society of Japan 8 (2): 21-33.

Klie W. 1939a. Ostracoden aus dem Kenia-Gebiet, vornehmlich von dessen Hochgebirgen. Internationale Revue der gesamten Hydrobiologie und Hydrographie 39 (1-2): 99-161.

Klie W. 1939b. Zur Kenntnis von Cypris balnearia Moniez (Ostracoda). Zoologischer Anzeiger 126 (11-12): 298-302.

Klie W. 1939c. Süßwasserostracoden aus Nordostbrasilien: 2. Die Gattung Chlamydotheca. Zoologischer Anzeiger 128 (5-6): 152-159.

Klie W. 1940. Süßwasser-Ostracoden aus Nordostbrasilien. 6: Cyprinae mit geißelförmiger Furka. Zoologischer Anzeiger 130 (3/4): 59-73.

Klugh A.B. 1923. A new Cyclocypris from Eastern Canada. Transactions of the Royal Canadian Institute 14: 337-342.

Koenders A., Martens K., Halse S. \& Schön I. 2012. Cryptic species of the Eucypris virens species complex (Ostracoda, Crustacea) from Europe have invaded Western Australia. Biological Invasions 14: 2187-2201. https://doi.org/10.1007/s10530-012-0224-y

Kovalenko A.L. 1976. Sovremennye Ostrakody Basseyna Dnestra (Recent Ostracoda from the Basin of the River Dnestr), Kishinev.

Kovalenko A.L. 1987. Bentocypria - novyi rod nadsemeystva Cypridacea (Crustacea, Ostracoda) (A new genus of the superfamily Cypridacea (Crustacea, Ostracoda). [In Russian]. In: Nevesskaya L.A. (ed.) Stratigrafiya Verkhnego Fanerozoya Moldavii (Sbornik Nauchnykh Trudov): 99-105. Shtiintsa, Kishinev.

Krstić N. 1977. The ostracod genus Tyrrhenocythere. In: Löffler H. \& D.L. Damielopol (eds) Aspects of ecology and zoogeography of Recent and fossil Ostracoda: 395-405. The Hague.

Krstić N. 1979. The Pliocene Ostracodes of Metohija, 4. Upper Pliocene of the locality Cabrat (Davovica): II. Other species of the genus Candona. Bulletin du Muséum d'Histoire naturelle, Belgrade série A 34: 169-178. [In Serbian.]

Krstić N. 1981. Quaternary of Orlovacha near Mol. Comptes rendus des séances de la Société Serbe de Géologie 1980: 87-90. [In Serbian.]

Krstić N. 2006. Pliocene ostracodes of the Paludinian beds in Pannonian Plain, Serbian Part. Herald of the Nature History Museum, Belgrade, Special Publication: 1-409. 
Külköylüoğlu O. \& Vinyard G.L. 2000. Distribution and ecology of freshwater Ostracoda (Crustacea) collected from springs of Nevada, Idaho, and Oregon: a preliminary report. Western North American Naturalist 60: 291-303.

Lindroth S. 1953. Taxonomic and geographical studies of the ostracod fauna in the inland waters of East Africa. Zoologiska Bidrag fran Uppsala 30 (1952): 43-156.

Löffler H. 1961. Beiträge zur Kenntnis der iranischen Binnengewässer 2: Regional-limnologische Studie mit besonderer Berücksichtigung der Crustaceenfauna. Internationale Revue der gesamten Hydrobiologie und Hydrographie 46 (3): 309-406.

Löffler H. 1963a. Zur Ostrakoden- und Copepodenfauna Ekuadors (mit Beschreibung 6 neuer Arten und 3 neuer Unterarten). Archiv für Hydrobiologie 59 (2): 196-234.

Löffler H. 1963b. Beiträge zur Fauna Austriaca. I. Die Ostrakodenfauna Österreichs. Sitzungsberichte der österreichischen Akademie der Wissenschaften, mathematisch-naturwissenschaftliche Klasse, Abt. 1 172 (3-5): 193-211.

Löffler H. 1967. Ostracoda. In: Illies J. (ed.) Limnofauna Europaea: 162-172. Stuttgart.

Lowndes A.G. 1931. Some rare and little-known British fresh-water ostracods. Report of the Marlborough College Natural History Society 79 (1930): 82-112.

Lowndes A.G. 1932. Report on the Ostracoda. Mr. Omer-Cooper's investigation of the Abyssinian fresh waters (Dr. Hugh Scott's Expedition). Proceedings of the Zoological Society of London 1932 (3): 677708.

Lilljeborg W. 1853. De Crustaceis ex ordinibus tribus: Cladocera, Ostracoda et Copepoda, in Scania occurrentibus. Lund.

Lüttig G. 1962. Zoologische und paläontologische Ostracoden-Systematik. Paläontologische Zeitschrift 36: 154-184. https://doi.org/10.1007/BF02987899

Maddocks R.F. 1992. Anchialine Cyprididae (Ostracoda) from the Galápagos Islands, with a review of the subfamily Paracypridinae. Zoological Journal of the Linnean Society of London 104: 1-29.

Malz H. 1973. Rudjakoviella Triebel (†), nom. nov., replaces Xenocypris Triebel, 1962 (Ostracoda). Senckenbergiana Lethaea 53 (6): 543.

Mandelstam M.I. 1962. In: Mandelstam M.I., Markova L.P., Rosyjeva T.R. \& Stepanaitys N.E. (eds) Ostracoda from Pliocene and Postpliocene deposits of Turkmenistan. Ashkhabad: 1-288. Ashkhabad. [In Russian.]

Margalef R. 1946. Algunos ostrácodos de las aguas continentales del NE de España. Publicaciones del Instituto de Biología Aplicada 2: 35-47.

Margalef R. 1948. Estudios sobre la vida en las aguas continentales de la región endorreica Manchega. Publicaciones del Instituto de Biología Aplicada 4 (1947): 5-51.

Margalef R. 1953. Los crustáceos de las aguas continentales Ibéricas. Biología de las Aguas Continentales 10: 1-243.

Marmonier P. \& Ward J. 1990. Superficial and interstitial Ostracoda of the South Platte River (Colorado, U.S.A.) - Systematics and biogeography. Stygologia 5 (4): 225-239.

Marmonier P., Meisch C. \& Danielopol D.L. 1989. A review of the genus Cavernocypris Hartmann (Ostracoda, Cypridopsinae): systematics, ecology and biogeography. Bulletin de la Société des Naturalistes luxembourgeois 89: 221-278. 
MEISCH C. et al., Global checklist of extant non-marine Ostracoda

Martens K. 1982. On a small collection of freshwater ostracods (Crustacea, Ostracoda) from Somalia, with a description of two new species. Monitore Zoologico Italiano n.s. 17 (5) (Supplemento): 149170.

Martens K. 1984. Annotated checklist of non-marine ostracods (Crustacea, Ostracoda) from African inland waters. Koninklijk Museum voor Midden-Afrika Tervuren-België, Zoologische Dokumentatie 20: $1-51$.

Martens K. 1985. Tanganyikacypridopsis gen. n. (Crustacea, Ostracoda) from Lake Tanganyika. Zoologica Scripta 14 (3): 221-230.

Martens K. 1986. Taxonomic revision of the subfamily Megalocypridinae Rome, 1965 (Crustacea, Ostracoda). Verhandelingen van de Koninklijke Academie voor Wetenschappen, Letteren en Schone Kunsten van België, Klasse der Wetenschappen 174: 1-79.

Martens K. 1989. On the systematic position of the Eucypris clavata-group, with a description of Trajancyris gen. nov. (Crustacea, Ostracoda). Archiv für Hydrobiologie, Supplement 83: 227-251.

Martens K. 1990a. Revision of African Limnocythere s.s. Brady, 1867 (Crustacea, Ostracoda), with special reference to the Rift Valley Lakes: morphology, taxonomy, evolution and (palaeo-)ecology. Archiv für Hydrobiologie, Supplemente 83 (4): 453-524.

Martens K. 1990b. Taxonomic revision of African Cypridini. Part I: the genera Cypris O.F. Müller, Pseudocypris Daday and Globocypris Klie (Crustacea, Ostracoda). Bulletin de l'Institut royal des Sciences naturelles de Belgique 60: 127-172.

Martens K. 1991. On a small collection of non-marine ostracods from Mongolia, with the description of a new species (Crustacea, Ostracoda). Miscellanea Zoologica Hungarica 6: 53-60.

Martens K. 1992a. Taxonomic revision of African Cypridini. Part 2. Description of Ramotha gen. nov. (Crustacea, Ostracoda). Annals of the South African Museum 102 (2): 91-130.

Martens K. 1992b. A reassessment of Paralimnocythere Carbonnel, 1965 (Crustacea, Ostracoda, Limnocytherinae), with a description of a new genus and two new species. Bulletin de l'Institut royal des Sciences naturelles de Belgique, Biologie 62: 125-158.

Martens K. 1993. The ostracod fauna of the old Lake Hula (Israel). Travaux scientifiques du Musée national d'Histoire naturelle de Luxembourg 19: 67-75.

Martens K. 1994. Ostracod speciation in ancient lakes: a review. Archiv für Hydrobiologie - Beiheft Ergebnisse der Limnologie 44: 203-222.

Martens K. 1996. On Korannacythere gen. nov. (Crustacea, Ostracoda), a new genus of temporary pool limnocytherids from southern Africa, with the description of three new species and a generic reassessment of the Limnocytherinae. Bulletin de l'Institut royal des Sciences naturelles de Belgique, Biologie 66: 51-72.

Martens K. 1997. Two new crenobiont ostracod genera (Crustacea, Ostracoda, Herpetocypridinae) from Africa and Asia Minor, with the description of a new species from dolomitic springs in South Africa. South African Journal of Science 93: 542-554.

Martens K. 2001a. Taxonomy of the Herpetocypridinae (Ostracoda, Cyprididae). Crustaceana 74: 295308.

Martens K. 2001b. Ostracoda. In: Day J.A., de Moor I.J., Stewart B.A. \& Louw A.E. (eds) Guides to the Freshwater Invertebrates of Southern Africa 3 Crustacea II: 9-77. Pretoria. 
Martens K. \& Behen F. 1994. A checklist of the Recent non-marine ostracods (Crustacea, Ostracoda) from the inland waters of South America and adjacent islands. Travaux scientifiques du Musée national d'histoire naturelle de Luxembourg 22: 1-82.

Martens K. \& Coomans A. 1990. Phylogeny and historical biogeography of the Megalocypridinae Rome, 1965; with an updated checklist of the subfamily. In: Whatley R. \& Maybury C. (eds) Proceedings of the 10th Symposium on Ostracoda, Aberystwyth, Wales: 545-556. Chapman \& Hall, London.

Martens K. \& Dumont H. 1984. The ostracod fauna (Crustacea, Ostracoda) of lake Donk (Flanders): A comparison between two surveys 20 years apart. Biologisch Jaarboek Dodonaea 52: 95-111.

Martens K., De Deckker P. \& Rossetti G. 2004. On a new terrestrial genus and species of Scottiinae (Crustacea, Ostracoda) from Australia, with a discussion on the phylogeny and the zoogeography of the subfamily. Zoologischer Anzeiger 243: 21-36. https://doi.org/10.1016/j.jcz.2004.05.001

Martens K. \& Rossetti G. 1997. On two new species of Darwinula Brady \& Robertson, 1885 (Crustacea, Ostracoda) from South African dolomitic springs. Bulletin de l'Institut royal des Sciences naturelles de Belgique, Biologie 67: 57-66.

Martens K. \& Savatenalinton S. 2011. A subjective checklist of the Recent, free-living, non-marine Ostracoda (Crustacea). Zootaxa 2855: 1-79.

Martens K. \& Toguebaye B.S. 1985. On the presence of Cypris subglobosa Sowerby, 1840 (Crustacea, Ostracoda) in Africa, with notes on the distribution of this species. Annales de la Société royale zoologique de Belgique 115 (2): 147-153.

Martens K. \& Wouters K. 1985. On Hemicypris dentatomarginata (Baird). Stereo-Atlas of Ostracod Shells 12 (23): 127-134.

Martens K., Meisch C. \& Marmonier P. 1991. On Klieopsis n. gen., with a redescription of Cypridopsis horai Klie, 1927 (Crustacea, Ostracoda). Bulletin de l'Institut royal des Sciences naturelles de Belgique, Biologie 61: 55-64.

Martens K., Davies B.R., Baxter A.J. \& Meadows M.E. 1996. A contribution to the taxonomy and ecology of the Ostracoda (Crustacea) from Verlorenvlei (Western Cape, South Africa). South African Journal of Zoology 31 (1): 23-36.

Martens K., Würdig N. \& Behen F. 1998. Maxillopoda. Non-marine Ostracoda. In: Young P.S. (ed.) Catalogue of Crustacea of Brazil: 45-65. Museu Nacional, Rio de Janeiro.

Martens K., Schwartz S.S., Meisch C. \& Blaustein L. 2002. Non-marine Ostracoda (Crustacea) of Mount Carmel (Israel), with taxonomic notes on Eucypridinae and circum-mediterranean Heterocypris. Israel Journal of Zoology 48: 53-70.

Martens K., Schön I., Meisch C. \& Horne D.J. 2008. Global diversity of ostracods (Ostracoda, Crustacea) in freshwater. Hydrobiologia 595: 185-193. https://doi.org/10.1007/s10750-007-9245-4

Masi L. 1906. Contributo alla sistematica delle Ilyocyprinae. Bollettino della Societá Zoologica Italiana seria 2, 7 (4-6): 133-146; (7-9): 249-268: 8 (1-3): 55-57.

Matzke-Karasz R., Nagler C. \& Hofmann S. 2014. The ostracod springtail - camera recordings of a previously undescribed high-speed escape jump in the genus Tanycypris (Ostracoda, Cypridoidea). Crustaceana 87: 1072-1094. https://doi.org/10.1163/15685403-00003343

Mazepova G.F. 1990. Rakushkovye rachki (Ostracoda) Baykala. Sibirskogo Otdelenija Akademii Nauk SSSR, Novosibirsk. [In Russian].

Mazzini I., Gliozzi E., Koci R., Soulie-Märsche I., Zanchetta G., Baneschi I., Sadori L., Giardini M., Van Welden A. \& Bushati S. 2015. Historical evolution and Middle to Late Holocene environmental changes 
MEISCH C. et al., Global checklist of extant non-marine Ostracoda

in Lake Shkodra (Albania): New evidence from micropaleontological analysis. Palaeogeography, Palaeoclimatology, Palaeoecology 419: 47-59. https://doi.org/10.1016/j.palaeo.2014.08.012

Mazzini I., Marrone F., Arculeo M. \& Rossetti G. 2017. Revision of Recent and fossil Mixtacandona Klie 1938 (Ostracoda, Candonidae) from Italy, with a description of a new species. Zootaxa 4221: 323-340. https://doi.org/10.11646/zootaxa.4221.3.3

McKenzie K.G. 1966a. Freshwater Ostracoda from North-Western Australia. Australian Journal of Marine and Freshwater Research 17 (2): 259-279.

McKenzie K.G. 1966b. Mytilocypris, a new ostracode genus from Tasmania. Papers and Proceedings of the Royal Society of Tasmania 100: 27-30.

McKenzie K.G. 1970. Synonymy of Cypris monstrifica Norman, 1862, and Ilyocyris ambigua Lowndes, 1931 (Ostracoda, Metacopina). Crustaceana 18: 109-110.

McKenzie K.G. 1971. Species list of South African freshwater Ostracoda with an appendix listing museum collections and some further determinations. Annals of the South African Museum 57: 157-213.

McKenzie K.G. 1972. Check List of Ostracoda recorded from the Indian Subcontinent and Ceylon (1840-1971). I-III. Melbourne.

McKenzie G.K. 1977. Illustrated generic key to South African continental Ostracoda. Annals of the South African Museum 74 (3): 45-103.

McKenzie K.G. 1980. A new subfamily from Gorong, Seram, Moluccas, with the description of Renaudcypris new genus (Crustacea, Ostracoda). Bulletin du Muséum national d'Histoire naturelle de Paris série 4: 2 (section A: 2): 507-5015.

McKenzie K.G. 1982a. Homoeomorphy: Persistent joker in the taxonomic pack, with the description of Bradleycypris gen. nov. In: Bate R.H., Robinson E. \& Sheppard L.M. (eds) Fossil and Recent Ostracods: 407-438, Ellis Horwood.

McKenzie K.G. 1982b. Description of a new cypridopsine genus (Crustacea: Ostracoda) from Campbell Island, with a key to the Cypridopsinae. Proceedings of the Biological Society of Washington 95 (4): 766-771.

McKenzie K.G. 1986. A comparative study of collections from the S.W. Pacific (Saipan to Tonga), with the descriptions of Gambiella caudata (Brady, 1890) and a new species of Pterobairdia (Ostracoda). Journal of Paleontology 5: 91-108. https://doi.org/10.1144/jm.5.1.91

McKenzie K.G. \& Pollard D.A. 1966. Ostracodes in the diets of fish from Lake Modewarre, near Geelong, Victoria. Newsletter of the Australian Society for Limnology 6: 14-17.

Medici M.C., Ceci M.E. \& Gliozzi E. 2011. Early Pliocene brackish and freshwater Ostracoda from the Valdelsa Basin (Tuscany, Central Italy). Revista Italiana di Paleontologia e Stratigrafia 117 (3): $473-500$.

Meisch C. 1984. Revision of the Recent Western Europe species of genus Potamocypris. Part I: species with short swimming setae on the second antennae. Travaux scientifiques du Musée d'Histoire naturelle de Luxembourg 3: 1-55.

Meisch C. 1985. Revision of the Recent West European species of the genus Potamocypris. Part II: species with long swimming setae on the second antennae. Travaux scientifiques du Musée d'Histoire naturelle de Luxembourg 6: 1-95.

Meisch C. 1993. Heterocypris reptans (Kaufmann, 1900), a rare freshwater ostracod new to France (Crustacea, Ostracoda). Bulletin de la Société des Naturalistes luxembourgeois 94: 209-218. 
Meisch C. 1996. Contribution to the taxonomy of Pseudocandona and four related genera, with the description of Schellencandona nov. gen., a list of the Candoninae genera, and a key to the European genera of the subfamily (Crustacea, Ostracoda). Bulletin de la Société des naturalistes luxembourgeois 97: 211-237.

Meisch C. 2000. Freshwater Ostracoda of Western and Central Europe. In: Schwoerbel J. \& Zwick P. (eds) Süßwasserfauna von Mitteleuropa 8/3. Spektrum Akademischer Verlag, Heidelberg, Berlin.

Meisch C. \& Broodbakker N. W. 1990. On Freshwater Ostracoda (Crustacea) collected on the Canary Islands. Beaufortia 41 (21): 151-157.

Meisch C. \& Broodbakker N.W. 1993. Freshwater Ostracoda (Crustacea) collected by Prof. J.H. Stock on the Canary and Cabo Verde Islands. With an annotated checklist of the freshwater Ostracoda of the Azores, Madeira, the Canary, the Selvagens and Cape Verde Islands. Travaux scientifiques du Musée national d'Histoire naturelle de Luxembourg 19: 3-47.

Meisch C. \& Forró L. 1998. Checklist of Recent Ostracoda (Crustacea) from Hungary. Miscellanea Zoologica Hungarica 11 (1997): 33-48.

Meisch C., Wouters K. \& Martens K. 1990. Liste annotée des Ostracodes actuels non-marins trouvés en France (Crustacea, Ostracoda). Travaux scientifiques du Musée national d'histoire naturelle de Luxembourg 15: 1-62.

Meisch C., Mary-Sasal N., Colin J.-P. \& Wouters K. 2007. Freshwater Ostracoda (Crustacea) collected from the islands of Futuna and Wallis, Pacific Ocean, with a checklist of the nonmarine Ostracoda of the Pacific Islands. Bulletin de la Société des Naturalistes luxembourgeois 108: 89-103.

Mestre A., Monrós J.S. \& Mesquita-Joanes F. 2014. A review of the Entocytheridae (Ostracoda) of the world: updated bibliographic and species checklists and global georeferenced database, with insights into host specificity and latitudinal patterns of species richness. Crustaceana 87: 923-951. https://doi.org/10.1163/15685403-00003337

Mischke S., Sun Z., Herzschuh U., Qiao Z. \& Sun N. 2010. An ostracod-inferred large Middle Pleistocene freshwater lake in the presently hyper-arid Qaidam Basin (NW China). Quaternary International 218: 74-85. https://doi.org/10.1016/j.quaint.2009.03.002

Müller G.W. 1898. Ergebnisse einer zoologischen Forschungsreise in Madagaskar und Ost-Afrika 18891895 von Dr. A. Voeltzkow: Die Ostracoden. Abhandlungen der Senckenbergischen Naturforschenden Gesellschaft 21 (2): 255-296.

Müller G.W. 1900. Deutschlands Süsswasser-Ostracoden. Zoologica, Original-Abhandlungen dem Gesamtgebiete der Zoologie 12: 1-112.

Müller G.W. 1901. Stenocypria nov. gen. Zoologischer Anzeiger 24: 571-572.

Müller G.W. 1903. Hamburgische Elb-Untersuchung. 3. Ostracoden. Mitteilungen aus dem naturhistorischen Museum in Hamburg 19: 163-167.

Müller G.W. 1912. Crustacea. Ostracoda. In: Schulze F.E. (ed.) Das Tierreich 31: 1-434. Reprinted 1966 by J. Cramer, Weinheim.

Müller O.F. 1776. Zoologiae danicae Prodromus, seu Animalium daniae et norvegiae indigenarum Characteres, Nomina, et Synonyma imprimis popularium. I-XXXXII: 1-282.

Müller O.F. 1785. Entomostraca seu Insecta testacea, quae in Aquis Daniae et Norvegiae reperit, descripsit et Iconibus illustravit. Lipsiae et Havniae (Leipzig and Copenhagen). 
Nagler C., Geist J. \& Matzke-KaraszR. 2014. Revision of the genus Tanycypris (Ostracoda, Cypricercinae) with the description of Tanycypris alfonsi $\mathrm{n}$. sp. and an identification key to the genus. Zootaxa 3821 (4): 401-424. https://doi.org/10.11646/zootaxa.3821.4.1

Namiotko T. \& Danielopol D.L. 2002. Morphology and phylogenetic affinities of Cryptocandona brehmi (Klie, 1934) (Ostracoda, Podocopida). Crustaceana 74: 1349-1363.

Namiotko T., Danielopol D.L., Pichler M. \& von Grafenstein U. 2009. Occurrence of an arctic ostracod species, Fabaeformiscandona harmsworthi (Scott, 1899) (Ostracoda, Candonidae) in late glacial sediments of Lake Mondsee (Austria). Crustaceana 82 (9): 1209-1212. https://doi.org/10.1163/156854009X454612

Namiotko T., Danielopol D.L., Belmecheri S., Gross M. \& von Grafenstein U. 2012. On the Leptocytheridae ostracods of the long-lived Lake Ohrid: A reappraisal of their taxonomic assignment and biogeographic origin. International Review of Hydrobiology 97: 356-374.

https://doi.org/10.1002/iroh.201211496

Namiotko T., Danielopol D.L., Meisch C., Gross M. \& Mori N. 2014. Redefinition of the genus Typhlocypris Vejdovský, 1882 (Ostracoda, Candonidae). Crustaceana 87: 952-984. https://doi.org/10.1163/15685403-00003338

Neale J.W. 1977. Ostracods from the rice fields of Sri Lanka (Ceylon). In: Löffler H. \& Danielopol D.L. (eds) Aspects of the Ecology and Zoogeography of Recent and Fossil Ostracoda. Sixth International Ostracod Symposium, Salfelden, Austria: 271-283. Dr. W. Junk, The Hague.

Negadaev-Nikonov K.N. 1967. Morphologie sowie paläobiologische und biostratigraphische Bedeutung Quartärer Limnocythere-Arten (Ostracoda, Arthropoda). Izvestiya Akademii Nauk Moldavskoy SSR (4: Paleontologiya i Strastigrafiya): 34-44. [In Russian.]

Negadaev-Nikonov K.N. 1968. On the phylogenetical development of species of the genus Limnocythere. Izvestiya Akademii Nauk Moldavskoy SSR seriya Biologicheskikh i Khimicheskykh Nauk 3: 3-9. [In Russian.]

Norman A.M. \& Brady G.S. 1909. The Crustacea of Northumberland and Durham. Transactions of the Newcastle Natural History Society 3: 252-417.

Okubo I. 1974. Freshwater Ostracoda from Japan - 10: Chrissia vittata sp. nov. from Japan (Ostracoda, Cyprididae). Proceedings of the Japanese Society of Systematic Zoology 10: 1-9.

Okubo I. 2004. Nihon tansui san kaimijinko rui ni tsuite. Kabushikigaisha Sanmon Insatsusho, Okayama: 1-72. [On Japanese freshwater ostracods, in Japanese, privately published.]

Ornellas L.P. \& Würdig N.L. 1983. Cyprideis salebrosa hartmanni (Ramirez, 1967), a new subspecies from Brasil and Argentina. Pesquisas 15: 94-112.

Pérez L., Lorenschat J., Brenner M., Scharf B. \& Schwalb A. 2010. Extant freshwater ostracodes (Crustacea: Ostracoda) from Lago Petén Itzá, Guatemala. Revista de Biología Tropical 58: 871-895. https://doi.org/10.15517/rbt.v58i2.5252

Peters D.J. \& Pugh J.E. 1999. On the entocytherid ostracods of the Brazos River basin and adjacent coastal region of Texas. Proceedings of the Biological Society of Washington 112: 338-351.

Petkovski T.K. 1964. Bemerkenswerte Entomostraken aus Jugoslavien. Acta Musei Macedonici Scientiarum Naturalium 9 (7): 147-182.

Petkovski T.K. 1976. Zwei neue und eine seltene Ostracoden-Art der Gattung Cypria Zenker aus Jugoslawien. Acta Musei Macedonici Scientiarum Naturalium 14 (7): 173-192. 
Petkovski T. \& Meisch C. 1996. Species of the genus Stenocypris Sars, 1889 from the rice-fields of Macedonia (Crustacea, Ostracoda). Travaux scientifiques du Musée national d'Histoire naturelle de Luxembourg 23: 57-85.

Petkovski T., Scharf B. \& Keyser D. 2000. New and little known ostracods of the genus Heterocypris (Crustacea, Ostracoda) from the Balkan Peninsula. Limnologica 30: 45-57.

https://doi.org/10.1016/S0075-9511(00)80042-0

Petkovski T., Scharf B. \& Keyser D. 2002. New and little known species of the genus Candona (Crustacea, Ostracoda) from Macedonia and other Balkan areas. Limnologica 32: 114-130. https://doi.org/10.1016/S0075-9511(02)80003-2

Petkovski T.K., ScharfB. \& Keyser D. 2016. Arctocypris fuhrmanni, n. gen., n. sp. (Crustacea, Ostracoda, Eucypridinae) from Spitsbergen (Norway). Zootaxa 4066 (2): 152-160.

https://doi.org/10.11646/zootaxa.4066.2.3

Pieri V., Martens M., Meisch C. \& Rossetti G. 2015. An annotated checklist of the Recent non-marine ostracods (Ostracoda: Crustacea) from Italy. Zootaxa 3919 (2): 271-305.

https://doi.org/10.11646/zootaxa.3919.2.3

Pinto I.D. \& Sanguinetti Y.T. 1962. A complete revision of the genera Bisulcocypris and Theriosynoecum (Ostracoda) with the world geographical and stratigraphical distribution (including Metacypris, Elpidium, Gomphycythere and Cytheridella). Publicaçao especial, Ecola de Geologia, Universidade do Rio Grande do Sul 4: 1-165.

Purper I. \& Würdig-Maciel 1974. Occurrence of Heterocypris incongruens (Ramdohr), 1808 - Ostracoda - in Rio Grande do Sul, Brazil. Discussion on the allied genera: Cyprinotus, Hemicypris, Homocypris and Eucypris. Pesquisas 3: 69-91.

Rasouli H., Scharf B., Meisch C. \& Aygen C. 2016. An updated checklist of the Recent non-marine Ostracoda (Crustacea) of Iran, with a redescription of Eucypris mareotica (Fischer, 1855). Zootaxa 4154 (3): 273-292. https://doi.org/10.11646/zootaxa.4154.3.3

Remy P. 1948. Description de Sphaeromicola cebennica n. sp., Ostracode Cythéride commensal de l'Isopode Cirolanide cavernicole Sphaeromides raymondi Dollfus. Bulletin mensuel de la Société linnéenne de Lyon 17 (7): 129-132.

Roessler E.W. 1990. Estudios sobre los ostrácodos de agua dulce en Colombia - 6. Parte 4: Estudio taxonómico del grupo 'Strandesia psittacea psittacea (Sars, 1901)'. Caldasia 16 (77): 215-230.

Rome D.R. 1962. Ostracodes. Exploration hydrobiologique du Lac Tanganika (1946-1947). Résultats scientifiques 3 (8), Institut royal des sciences naturelles de Belgique, Bruxelles.

Rome D.R. 1965. Crustacea: Ostracoda. In: Hanstrom B., Brinck P. \& Rudebeck G. (eds) South African Animal Life. Results of the Lund University Expedition in 1950-1951 11: 9-58.

Rossetti G. \& Martens K. 1998. Taxonomic revision of the Recent and Holocene representatives of the Family Darwinulidae (Crustacea, Ostracoda), with a redescription of three new genera. Bulletin de l'Institut royal des Sciences naturelles de Belgique, Biologie 68: 55-110.

Sandberg P.A. 1964. The ostracod genus Cyprideis in the Americas. Acta Universitatis Stockholmiensis, Stockholm Contributions in Geology 12: 1-178.

Sandberg P.A. \& Plusquellec P.L. 1974. Notes on the anatomy and passive dispersal of Cyprideis (Cytheracea, Ostracoda). Geoscience and Man 6: 1-26.

Sars G.O. 1866. Oversigt af Norges marine Ostracoder. Forhandliger I Videnskabs-Selskabet I Christiana 1865, Christiania. 
MEISCH C. et al., Global checklist of extant non-marine Ostracoda

Sars G.O. 1890. Oversigt af Norges Crustaceer, med foreløbige Bemaerkninger over de nye eller mindre bekjendte Arter: 2 (Branchipoda, Ostracoda, Cirripedia). Forhandlinger $i$ Videnskabs-selskabet $i$ Christiania 1890: 1-80.

Sars G.O. 1894. Contributions to the knowledge of the fresh-water Entomostraca of New Zealand as shown by artificial hatching from dried mud. Skrifter I Videnskkabs-Selskabet, I. MathematiskNaturvidenskabs Klasse 1894 (5): 1-62.

Sars G.O. 1896. On freshwater Entomostraca from the neigbourhood of Sydney, partly raised from dried mud. Archiv for Mathematik og Naturvidenskab 18 (3): 1-81.

Sars G.O. 1903a. On the Crustacean Fauna of Central Asia. Part 3: Copepoda and Ostracoda. Annuaire du Musée zoologique de l'Académie impériale de St.-Pétersbourg 8 (2): 195-264.

Sars G.O. 1903b. Fresh-water Entomostraca from China and Sumatra. Archiv for Mathematik og Naturvidenskab 25 (8): 3-44.

Sars G.O. 1910. Zoological results of the third Tanganyika expedition, conducted by Dr. W.A. Cunnington, 1904-1905. Report on the Ostracoda. Proceedings of the Zoological Society of London 1910 (2): 732-760.

Sars G.O 1924a. The fresh-water Entomostraca of the Cape Province (Union of South Africa). Part 2: Ostracoda. Annals of the South African Museum 20 (2): 105-193.

Sars G.O 1924b. Contributions to a knowledge of the fauna of South-West Africa. I: Crustacea Entomostraca, Ostracoda. Annals of the South African Museum 20 (3): 195-211.

Sars G.O. 1925. An Account of the Crustacea of Norway with short Descriptions and Figures of all the Species. 9. Ostracoda (3-10): 73-208, tables 33-96.

Sars G.O. 1926. Freshwater Ostracoda from Canada and Alaska. Report of the Canadian Arctic Expedition 1913-1918: 7 (Crustacea), Part 1 (Ostracoda): 1-23.

Savatenalinton S. 2015. On three new species of non-marine ostracods (Crustacea: Ostracoda) from Northeast Thailand. Zootaxa 3914: 275-300. https://doi.org/10.11646/zootaxa.3914.3.3

Savatenalinton S. \& Martens K. 2009a. Generic revision of Cypricercinae McKenzie, 1971 (Crustacea, Ostracoda), with the description of three new genera and one new species and a phylogenetic analysis of the subfamily. Hydrobiologia 632: 1-48. https://doi.org/10.1007/s10750-009-9826-5

Savatenalinton S. \& Martens K. 2009b. Redescription of the type species of Strandesia Stuhlmann, 1888 and Cypricercus Sars, 1895 (Crustacea, Ostracoda), with a description of a new species of Cypricercus from South Africa. Zootaxa 2007: 1-42.

Savatenalinton S. \& Martens K. 2010. On the subfamily Cypricercinae McKenzie, 1971 (Crustacea, Ostracoda) from Thailand, with the description of six new species. Zootaxa 2379: 1-77.

Savatenalinton S. \& Suttajit M. 2016. A checklist of Recent non-marine ostracods (Crustacea, Ostracoda) from Thailand, including descriptions of two new species. Zootaxa 4067: 1-34. https://doi.org/10.11646/zootaxa.4067.1.1

Schäfer H.W. 1952. Über Süßwasser-Ostracoden aus der Türkei. Hidrobiologi, Istanbul Üniversitesi fen Fakültesi Hidrobiologi Araştırma Enstitüsü Yayınlarındam, ser. B 1 (1): 7-32.

Schön I. \& Martens K. 1998. Sex determination in non-marine ostracods. In: Martens K. (ed.) Sex and parthenogenesis. Evolutionary ecology of reproductive modes in non-marine ostracods: 25-36. Backhuys Publishers, Leiden, The Netherlands. 
Schornikov E.I. 1966. Ostrakody Chernogo I Azovskogo Morey (Ostracoda from the Black Sea and the Sea of Azov). Avtoreferat Dissertatsii Na Soiskanie Uchenoy Stepeni Kandidata Biologi - Cheskikh Nauk, Leningradskiy Gosudarstvennyy Pedagogicheskiy Insitut: 1-21. [In Russian.]

Schornikov E.I. 1981. Tyrrhenocythere amnicola (Crustacea) - Politipicheskiy vid ostrakod v Kaynozoe Yuga SSSR (Tyrrhenocythere amnicola (Crustacea) - A polytypical ostracode species from the Cenozoic of the south of the USSR.) [In Russian]. In: Bragina L.F. (ed.) Biostratigrafiya Antropogena I Neogena Yugo-Zapada SSSR: 107-122. Akademiya Nauk Moldavskoy SSR, Otdel Paleontologii i Biostratigrafii, Kishinev.

Schornikov E.I. 2007. Modern and fossil (Quaternary) Ostracoda from high mountainous Tien Shan lake basins Sankul and Chatyrkul. In: Romanovsky V.V. (ed.) Climate, Glaciers, Lakes of Tien-Shan: Journey to the Past: 110-140. The Institute of Water Problems and Hydropower, Bishkek, Kyrgyzstan. [In Russian.]

Schornikov E.I. \& Trebukhova Y.A. 2001. Ostracods of brackish and fresh waters of southwestern coast of Peter the Great Bay. The State of Environment and Biota of the Southwestern Part of Peter the Great Bay and the Tumen River Mouth 3: 56-83.

Sharpe R.W. 1897. Contribution to a knowledge of the North American fresh-water Ostracoda included in the families Cytheridae and Cyprididae. Bulletin of the Illinois State Laboratory of Natural History 4: 414-484.

Sharpe R.W. 1910. On some Ostracoda, mostly new, in the collection of the United States National Museum. Proceedings of the United States National Museum 38 (1750): 335-341.

Sharpe R.W. 1918. The Ostracoda. In: Ward H.B. \& Whipple G.C. (eds) Fresh-Water Biology: 790828. Wiley, New York City.

Shearn R., Koenders A., Schön I., Halse S. \& Martens K. 2017. On the affinity of Isocypridinae and Herpetocypridinae, with redescriptions of four species of Ilyodromus Sars, 1894 (Crustacea, Ostracoda). Zootaxa 4318: 47-81. https://doi.org/10.11646/zootaxa.4318.1.2

Smith A.J. \& Horne D.J. 2016. Class Ostracoda. In: Thorp J.H. \& Covich A.P. (eds) Ecology and General Biology: Thorp and Covich's Freshwater Invertebrates, Volume 2. Academic Press (Elsevier), Burlington.

Smith R.J. \& Janz H. 2008. Recent species of the Family Candonidae (Ostracoda, Crustacea) from the ancient Lake Biwa, Central Japan. Journal of Natural History 42: 2865-2922. https://doi.org/10.1080/00222930802361030

Smith R.J., Janz H. \& Okubo I. 2011. Recent Cyprididae and Ilyocyprididae (Crustacea: Ostracoda) from Lake Biwa, Japan, including a summary of the lake's ostracod fauna. Zootaxa 2874: 1-37.

Sohn I.G. \& Kornicker L.S. 1973. Morphology of Cypretta kawatai Sohn and Kornicker, 1972 (Crustacea, Ostracoda), with a discussion of the genus. Smithsonian Contributions to Zoology 141: 1-28.

Stancheva M. 1968. New data on the subfamily Leptocytherinae Hanai, 1957. Izvestiya na Geologicheskiya Instituut, ser. Paleontologiya 17: 37-48.

Stephenson M.B. 1938. Miocene and Pliocene Ostracoda of the genus Cytheridea from Florida. Journal of Paleontology 12 (2): 127-148.

Straub E.W. 1952. Mikropaläontologische Untersuchungen im Tertiär zwischen Ehingen und Ulm an der Donau. Geologisches Jahrbuch 66: 433-523.

Swain F.M. 1995. Ostracoda from Holocene and Pleistocene lake sediments of Minnesota. Revista Española de Micropaleontología 27 (3): 15-67. 
MEISCH C. et al., Global checklist of extant non-marine Ostracoda

Swain F.M. 1999. Fossil nonmarine Ostracoda of the United States. Developments in Palaeontology and Stratigraphy 16: 1-401.

Sywula T. 1966. Notes on Ostracoda. I. On some Polish species. Bulletin de l'Académie polonaise des Sciences cl. 213 (11-12): 647-652.

Sywula T. 1974. Małżorackzki (Ostracoda). Fauna Słodkowodna Polski 24, Polska Academia Nauk, Warszwawa/Poznan. [In Polish].

Tétart J. 1985. Description de la carapace des ostracodes d'eau douce en France. II. Famille des Cyprididae (genres Cyclocypris, Cypria, Cypricercus, Eucypris). Spixiana 8: 171-195.

Tressler W.L. 1947. A check list of the known species of North American freshwater Ostracoda. American Midland Naturalist 38 (3): 698-707.

Triebel E. 1939. Zur Ostracoden-Fauna von Brasilien. Senckenbergiana 21 (5-6): 363-374.

Triebel E. 1959. Zur Kenntnis der Ostracoden-Gattungen Isocypris und Dolerocypris. Senckenbergiana biologica 40 (3-4): 155-170.

Triebel E. 1973. In: Malz H. (ed.) Rudjakoviella Triebel, nom. nov., replaces Xenocypris Triebel, 1962 (Ostracoda). Senckenbergiana lethaea 53 (6): 543.

Turner C.H. 1895. Fresh-water Ostracoda of the United States. Geological and Natural History Survey of Minnesota, Zoological Series 2: 277-337.

Van den Bold W.A. 1963. Upper Miocene and Pliocene Ostracoda of Trinidad. Micropaleontology 9 (4): 361-424.

Van der Meeren T., Khand Y. \& Martens K. 2009. On Recent species of Tonnacypris Diebel \& Pietrzeniuk, 1975 (Crustacea, Ostracoda), with new species descriptions from Mongolia. Zootaxa 2015: 1-41.

Vávra W. 1891. Monographie der Ostracoden Böhmens. Archiv der naturwissenschaftlichen Landesdurchforschung von Böhmen 8 (3) (I-IV): 1-116.

Vávra W. 1897. Die Süßwasser-Ostracoden Deutsch-Ost-Afrikas. In: Möbius K. (ed.) Die Tierwelt OstAfrikas und der Nachbargebiete 4 (2-3): 1-28.

Vávra W. 1901. Die Ostracoden vom Bismarck-Archipel. Archiv für Naturgeschichte 67: 179-186.

Victor R. \& Fernando C.H. 1979a. On some freshwater ostracod type specimens from Indonesia. Canadian Journal of Zoology 57: 6-12. https://doi.org/10.1139/z79-002

Victor R. \& Fernando C.H. 1979b. The freshwater ostracods (Crustacea: Ostracoda) of India. Records of the Zoological Survey of India 74 (2): 147-242.

Victor R. \& Fernando C.H. 1980. On Heterocypris makua (Tressler, 1937), a freshwater ostracod (Crustacea: Ostracoda) from the Hawaiian Islands, with notes on the other species of the genus. Canadian Journal of Zoology 58 (7): 1288-1297. https://doi.org/10.1139/z80-180

Victor R. \& Fernando C.H. 1981. Freshwater ostracods (Crustacea, Ostracoda) of the subfamily Dolerocypridinae Triebel, 1961 from Southern Asia. Zoological Journal of the Linnean Society 72: 107-116. https://doi.org/10.1111/j.1096-3642.1981.tb01654.x

Wagner C.W. 1957. Sur les ostracodes du Quaternaire récent des Pays-Bas et leur utilisation dans l'étude géologique des dépôts holocènes. Mouton \& Co., The Hague.

Wetterich S., Kuzmina S., Andreev A.A., Kienast F., Meyer H., Schirrmeister L., Kuznetsova T. \& Sierralta M. 2008a. Palaeoenvironmental dynamics inferred from late Quaternary permafrost deposits on Kurungnakh Island, Lena Delta, Northeast Siberia, Russia. Quaternary Science Reviews 27: 15231540. https://doi.org/10.1016/j.quascirev.2008.04.007 
Wetterich S., Schirrmeister L., Meyer H., Viehberg F.A. \& Mackensen A. 2008b. Arctic freshwater ostracods from modern periglacial environments in the Lena River Delta (Siberian Arctic, Russia): geochemical applications for palaeoenvironmental reconstructions. Journal of Paleolimnology 39: 427449. https://doi.org/10.1007/s10933-007-9122-1

Whatley R.C., Bajpai S. \& Whittaker J.E. 2003. The identity of the non-marine ostracod Cypris subglobosa Sowerby from the intertrappean deposits of peninsular India. Palaeontology 46: 1281-1296. https://doi.org/10.1046/j.0031-0239.2003.00341.x

Wilkinson I.P., Bubikyan S.A., Gulakyan S.Z. 2005. The impact of late Holocene environmental change on lacustrine Ostracoda in Armenia. Palaeogeography, Palaeoclimatology, Palaeoecology 225: 187202. https://doi.org/10.1016/j.palaeo.2005.06.010

Williams B.W. \& Weaver P.G. 2018. A historical review of the taxonomy and classification of Entocytheridae (Crustacea: Ostracoda: Podocopida). Zootaxa 4448: 1-129.

https://doi.org/10.11646/zootaxa.4448.1.1

Wouters K. 1983. Contributions to the study of Belgian Ostracoda. 1. The Ostracoda from the environs of Buzenol (Gaume District, Belgium). Bulletin de l'Institut royal des Sciences naturelles de Belgique, Biologie 55 (4): 1-9.

Wouters K. 1986. A new Renaudcypris (Crustacea: Ostracoda) from Lake Taal (Philippine Islands). Bulletin de l'Institut royal des Sciences naturelles de Belgique, Biologie 56: 125-129.

Wouters K. 1988. On Romecytheridea tenuisculpta (Rome). Stereo-Atlas of Ostracod Shells 15 (22): $97-100$.

Wouters K. 1989. Check-list of the recent non-marine Ostracoda (Crustacea) of Belgium. Comptes rendus du symposium "Invertébrés de Belgique”: 153-158.

Wouters K. 1994. In: Martens K. \& Behen F. (eds) A checklist of the Recent non-marine ostracods (Crustacea, Ostracoda) from the inland waters of South America and adjacent islands. Travaux scientifiques du Musée national d'histoire naturelle de Luxembourg 22: 1-81.

Wouters K. 1998. A new thalassocypridine genus (Crustacea, Ostracoda) from brackish waters in the Indian and Pacific Oceans, with the description of a new species. Bulletin de l'Institut royal des Sciences naturelles de Belgique, Biologie 68: 111-122.

Wouters K. 2001. On the genera Dolerocypria and Hansacypris (Crustacea, Ostracoda), with the description of three new species from Papua New Guinea. Bulletin de l'Institut royal des Sciences naturelles de Belgique, Biologie 71: 101-112.

Wouters K. 2002. On the distribution of Cyprideis torosa (Jones) (Crustacea, Ostracoda) in Africa, with the discussion of a new record from the Seychelles. Bulletin de l'Institut royal des Sciences naturelles de Belgique, Biologie 72: 131-140.

Wouters K. \& Martens K. 1992. Contribution to the knowledge of Tanganyikan cytheraceans, with description of Mesocyprideis nom. nov. (Crustacea, Ostracoda). Bulletin de l'Institut royal des Sciences naturelles de Belgique, Biologie 62: 159-166.

Wouters K. \& Martens K. 1994. Contribution to the knowledge of the Cyprideis species flock (Crustacea: Ostracoda) of Lake Tanganyika, with the description of three new species. Bulletin de l'Institut royal des Sciences naturelles de Belgique, Biologie 64: 111-128.

Wrozyna C., Frenzel P., Xie M., Zhu L. \& Schwalb A. 2009. A taxonomical and ecological overview of Recent and Holocene ostracodes of the Nam Co Region, Southern Tibet. Quaternary Science 29 (4): 665-677. 
Würdig N.L. \& Pinto I.D. 1990. Diaphanocypris, a new ostracod genus occurring in South and Central America. Pesquisas, Instituto de Geociencias, Universidade federal do Rio Grande do Sul 17 (1-2): $31-38$.

Yoo H., Cohuo S., Macario-Gonzalez L. \& Karanovic I. 2017. A new freshwater ostracod genus from the northern Neotropical region and its phylogenetic position in the family Cyprididae (Podocopida). Zoologischer Anzeiger 266: 196-215. https://doi.org/10.1016/j.jcz.2016.09.003

Zhai D. \& Zhao W. 2014. On some Recent non-marine ostracods from northern China, with description of one new species. Crustaceana 87 (8-9): 985-1026. https://doi.org/10.1163/15685403-00003339

Manuscript received: 4 June 2018

Manuscript accepted: 19 September 2018

Published on: 25 January 2019

Topic editor: Rudy Jocqué

Desk editor: Kristiaan Hoedemakers

Printed versions of all papers are also deposited in the libraries of the institutes that are members of the EJT consortium: Muséum national d'Histoire naturelle, Paris, France; Meise Botanic Garden, Belgium; Royal Museum for Central Africa, Tervuren, Belgium; Natural History Museum, London, United Kingdom; Royal Belgian Institute of Natural Sciences, Brussels, Belgium; Natural History Museum of Denmark, Copenhagen, Denmark; Naturalis Biodiversity Center, Leiden, the Netherlands; Museo Nacional de Ciencias Naturales-CSIC, Madrid, Spain; Real Jardín Botánico de Madrid CSIC, Spain; Zoological Research Museum Alexander Koenig, Bonn, Germany. 